NISTIR 7355

\title{
Development of a Fluorescence Based Measurement Technique to Quantify Water Contaminants at Pipe Surfaces \\ During Flow
}

\author{
Mark A. Kedzierski
}

National Institute of Standards and Technology

Technology Administration, U.S. Department of Commerce 

NISTIR 7355

\title{
Development of a Fluorescence Based Measurement Technique to Quantify Water Contaminants at Pipe Surfaces During Flow
}

\author{
Mark A. Kedzierski
}

U.S DEPARTMENT OF COMMERCE National Institute of Standard and Technology

Building Environment Division Building and Fire Research Laboratory

Gaithersburg, MD 20899-8631 


\title{
Development of a Fluorescence Based Measurement Technique to Quantify Water Contaminants at Pipe Surfaces During Flow
}

\author{
M. A. Kedzierski \\ National Institute of Standards and Technology \\ Bldg. 226, Rm B114 \\ Gaithersburg, MD 20899 \\ Phone: (301) 975-5282 \\ Fax: (301) 975-8973
}

\begin{abstract}
This paper provides a detailed account of the development of a fluorescence based measurement technique for measuring the mass of contaminant on solid surfaces in the presence of water flow. A test apparatus was designed and developed for the purpose of studying adsorption and desorption of diesel to and from a copper test surface in the presence of contaminated and fresh water flow, respectively. A calibration technique was developed to correlate the measured fluorescence intensity to the mass of diesel adsorbed per unit surface area (the excess surface density) and the bulk concentration of the diesel in the flow. Both bulk composition and the excess surface density measurements were achieved via a traverse of the fluorescent measurement probe perpendicular to the test surface. Two nominal bulk mass fractions $(0.2 \%$ and $0.3 \%)$ were tested each for five different Reynolds numbers between zero and 7000. Measurements for a given condition were made over a period of approximately $200 \mathrm{~h}$. The measured diesel excess surface density varied between zero and $0.02 \mathrm{~kg} / \mathrm{m}^{2}$ for the variation in the bulk mass fraction and Reynolds number of the flow. Normalized Freundlich constants were calculated for the various bulk mass fractions and Reynolds numbers.
\end{abstract}

Keywords: adsorption, contaminant, diesel, excess layer, fluorescence, measurement technique, sorption, water 


\section{LIST OF TABLES}

ABSTRACT

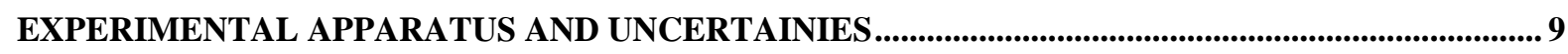

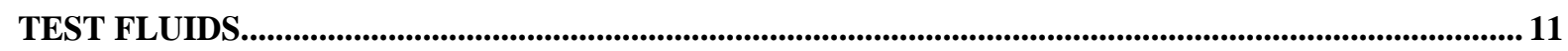

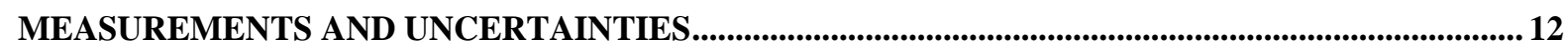

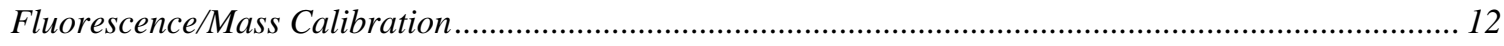

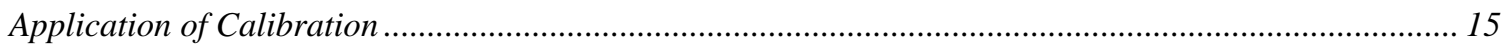

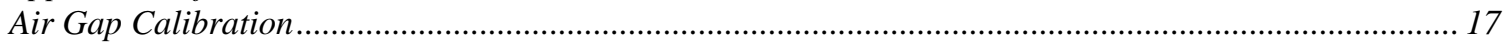

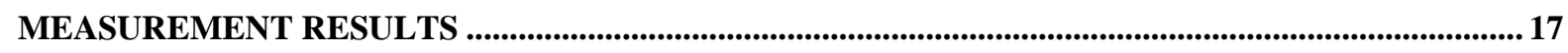

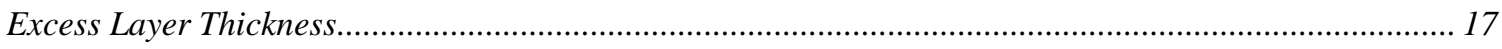

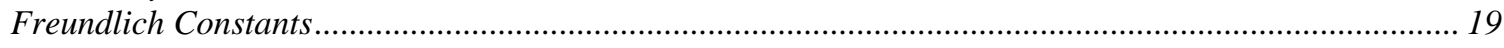

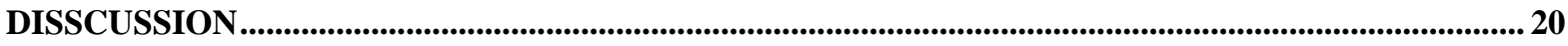

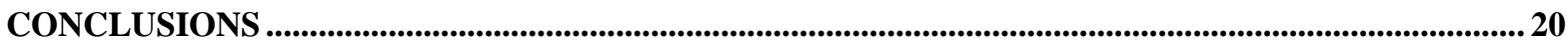

NOMENCLATURE

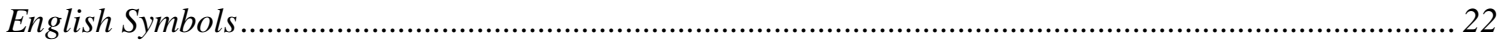

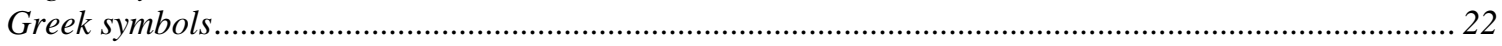

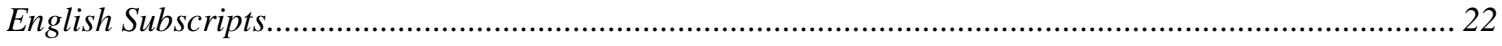

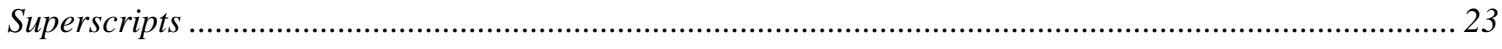

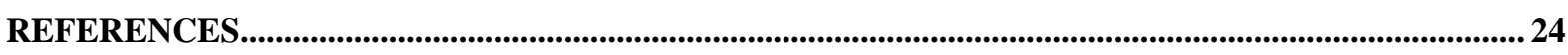

APPENDIX A: EXCITATION AND EMISSION WAVELENGTHS ........................................................ 38

APPENDIX B: DIESEL PROPERTIES ........................................................................................................... 40

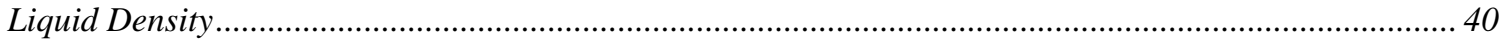

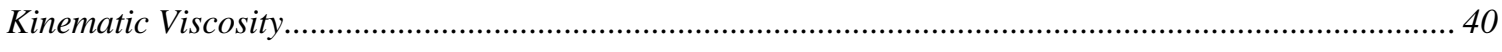

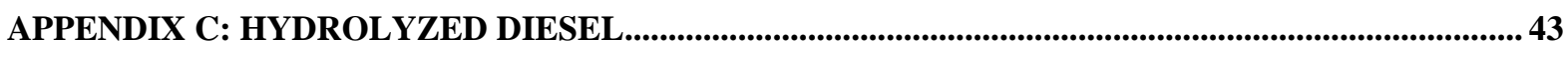

APPENDIX D: FLUORESCENCE TEMPERATURE DEPENDENCE........................................................ 45

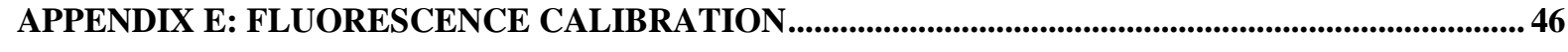

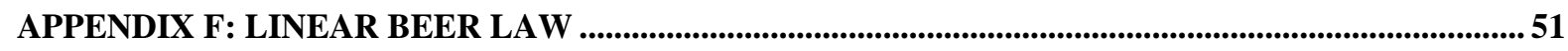

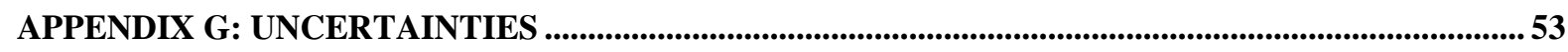

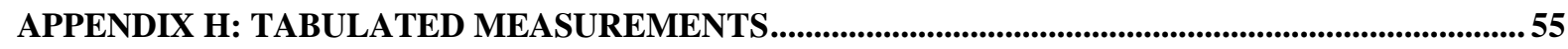

APPENDIX I: SPECTROFLUOROMETER CHECK ................................................................................ 81 


\section{LIST OF FIGURES}

Fig. 1 Schematic of test loop ........................................................................................................... 25

Fig. 2 Schematic of spectrofluorometer, test section, and linear positioning device...... 26

Fig. 3 Schematic of right angle spectrofluorometer.................................................... 27

Fig. 4 Cross-sectional illustration of test section during contamination and flushing... 28

Fig. 5 Schematic of fluorescence/composition calibration jar .................................. 27

Fig. 6 Overall calibration of Beer-Lambert Bougher law for diesel on copper disk ...... 30

Fig. 7 Demonstration of excess layer thickness measurement ......................................... 31

Fig. 8 Effect of exposure time and flow rate on thickness of the diesel excess layer for a

$0.2 \%$ bulk freestream mass fraction.................................................................................. 32

Fig. 9 Effect of exposure time and flow rate on thickness of the diesel excess layer for a

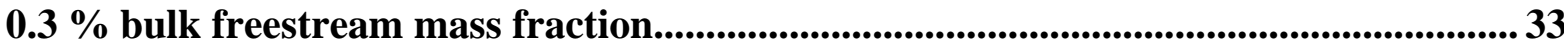

Fig. 10 Diesel excess layer thickness as a function of Re for water/diesel (99.8/0.2) ...... 34

Fig. 11 Diesel excess layer thickness as a function of Re for water/diesel (99.7/0.3) ...... 35

Fig. 12 Normalized Fruendlich constants for diesel adsorption to an oxidized Cu disk

from diesel contaminated water........................................................................... 36

Fig. A.1 Emission and excitation spectra for diesel ................................................... 38

Fig. A.2 Filtered excitation and emission spectra for diesel ....................................... 39

Fig. B.1 Measured liquid density of diesel and fit ..................................................4 41

Fig. C.1 Fluorescent emission spectra for pure diesel and hydrolyzed reservoir test

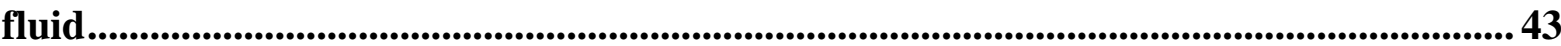

Fig. D.1 Temperature dependence of diesel fluorescence............................................ 45

Fig. E.1 Calibration of diesel fluorescence against diesel mass fraction for different runs

48

Fig. E.2 Filtered excitation and emission spectra for diesel ......................................... 49

Fig. E.3 Calibration of diesel fluorescence intensity for fixed film thickness and air gap

between quartz tube and liquid film ................................................................................. 50

Fig. F.1 Absorbance of diesel for calibration measurements as a function of mass

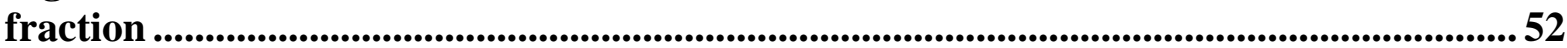

Fig. G.1 Relative uncertainty of le for $95 \%$ confidence level and $x b=0.2 \% \ldots \ldots . . . . . .55$

Fig. G.2 Relative uncertainty of le for $95 \%$ confidence level and $x b=0.3 \%$.............. 54

Fig. I.1 Verification of spectrofluorometer wavelength with Mercury standard.......... 82 


\section{LIST OF TABLES}

Table B.1 Diesel liquid density measurements (file:DieDen.dat)...................................... 42

Table B.2 Diesel \#2 liquid kinematic viscosity measurements (Simplex, 2006).............. 42

Table H.1.1 Diesel contamination on oxidized copper surface for $R e=0$ and $x_{b}=0.2 \%$

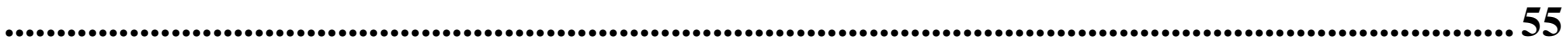

Table H.1.2 Diesel contamination on oxidized copper surface for $R e=1900$ and $x_{\mathrm{b}}=0.2 \%$

Table H.1.3 Diesel contamination on oxidized copper surface for $R e=3200$ and $x_{\mathrm{b}}=0.2 \%$

Table H.1.4 Diesel contamination on oxidized copper surface for $R e=4600$ and $x_{\mathrm{b}}=0.2 \%$................................................................................................................................ 58

Table H.1.5 Diesel contamination on oxidized copper surface for $R e=7000$ and $x_{\mathrm{b}}=0.2 \%$

Table $\mathrm{H} 1.6$ Tap water flushing after $\mathrm{Re}=4600$ contamination tests at $x_{\mathrm{b}}=0.2 \%$

Table H.1.7 Diesel contamination on oxidized copper surface for $R e=0$ and $x_{b}=0.3 \%$

Table H.1.8 Diesel contamination on oxidized copper surface for $R e=2000$ and $x_{\mathrm{b}}=0.3 \%$ 61

Table H.1.9 Diesel contamination on oxidized copper surface for $R e=4000$ and $x_{\mathrm{b}}=0.3 \%$

Table H.1.10 Diesel contamination on oxidized copper surface for $\mathrm{Re}=\mathbf{5 0 0 0}$ and $x_{\mathrm{b}}=0.3 \%$

Table H.1.11 Diesel contamination on oxidized copper surface for $R e=7000$ and $x b=$

$0.3 \%$

Table H.1.12 Tap water flushing after $R e=5000$ contamination tests at $x_{b}=0.3 \% \ldots .66$

Table H.1.13 Tap water flushing after $R e=7000$ contamination tests at $x_{b}=0.3 \% \ldots 67$

Table H.2.1 Diesel contamination on oxidized copper surface for $R e=0$ and $x_{b}=0.2 \%$

Table H.2.2 Diesel contamination on oxidized copper surface for $R e=1900$ and $x_{\mathrm{b}}=0.2 \%$

Table H.2.3 Diesel contamination on oxidized copper surface for $R e=3200$ and $x_{\mathrm{b}}=0.2 \%$

Table H.2.4 Diesel contamination on oxidized copper surface for $R e=4600$ and $x_{\mathrm{b}}=0.2 \%$

Table H.2.5 Diesel contamination on oxidized copper surface for $\mathbf{R e}=\mathbf{7 0 0 0}$ and $x_{\mathrm{b}}=0.2 \%$

Table H.2.6 Tap water flushing after $R e=4600$ contamination tests at $x_{b}=0.2 \% \ldots . . .73$

Table H.2.7 Diesel contamination on oxidized copper surface for $R e=0$ and $x_{b}=0.3 \%$

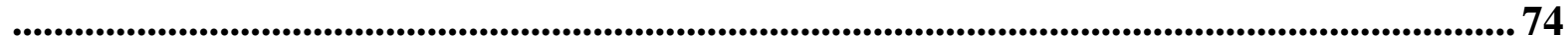

Table H.2.8 Diesel contamination on oxidized copper surface for $\mathbf{R e}=\mathbf{2 0 0 0}$ and $x_{\mathrm{b}}=0.3 \%$........................................................................................................... 75

Table H.2.9 Diesel contamination on oxidized copper surface for $R e=4000$ and $x_{\mathrm{b}}=0.3 \%$ 76

Table H.2.10 Diesel contamination on oxidized copper surface for $\mathbf{R e}=\mathbf{5 0 0 0}$ and $x_{b}=0.3 \%$ 
Table H.2.11 Diesel contamination on oxidized copper surface for $\mathbf{R e}=\mathbf{7 0 0 0}$ and

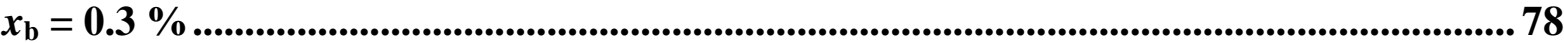

Table H.2.12 Tap water flushing after $R e=5000$ contamination tests at $x_{b}=0.3 \% \ldots .79$

Table H.2.13 Tap water flushing after $R e=7000$ contamination tests at $x_{b}=0.3 \%$

(file:flsh6c2.tb2) .........................................................................................................80

Table I.1 Calibration check of spectrofluorometer against Mercury lamp.................. 881 


\section{INTRODUCTION}

Since the signing of the Executive Order establishing the Office of Homeland Security, Federal agencies have been working on ways to improve the security of the general public. One way in which the National Institute of Standards and Technology (NIST) is doing its part is by helping the U.S. Environmental Protection Agency (EPA) devise ways to safeguard the nation's drinking water supply. EPA is conducting potable water research with NIST on six different efforts. This report describes one of those efforts designed to fundamentally understand the attachment and detachment mechanisms of contaminants to solid plumbing materials under dynamic water flow conditions. The results of this work provide EPA with an investigative tool to support the development of a response to water contamination events and a potential detection technique for timely warning of such events.

The purpose of this study is to apply a NIST fluorescence based measurement technique that was developed for measuring the mass of lubricant at the wall during boiling of refrigerants (Kedzierski, 2001) to measuring the mass of diesel on a copper pipe surface in the presence of flowing water/diesel mixture. In this way, we not only gain vital fundamental modeling information but we lay the groundwork for a possible early detection/monitoring system for sticky contaminants. Two major efforts have been focused toward the development of an in situ fluorescent measurement technique. First, a calibration technique was developed specifically for quantifying the amount of diesel on a copper pipe surface. Second, a water loop was designed and constructed consisting of a test chamber for subjecting small samples of pipe substrate materials to known concentrations of diesel/water solutions under controlled dynamic flow conditions. These two efforts have formed the foundation for future work that will focus on using the water loop and the calibration technique to measure the accumulation and removal of diesel as a function of free-stream diesel concentration and flow rate.

Commercial diesel was used rather than a chemically simpler surrogate in order to demonstrate the use of the technique with an actual potential contaminant. Diesel was also a desirable test contaminant because it has been found to exhibit a strong fluorescence. However, because of the complexity and the variability of diesel, the diesel for the project was restricted to a single batch. In this way, we can ensure the consistency of the properties of pure diesel ${ }^{1}$ such as its liquid density and fluorescence characteristics.

\section{EXPERIMENTAL APPARATUS AND UNCERTAINIES}

The standard uncertainty $\left(u_{\mathrm{i}}\right)$ is the positive square root of the estimated variance $u_{\mathrm{i}}{ }^{2}$. The individual standard uncertainties are combined to obtain the expanded uncertainty $(U)$, which is calculated from the law of propagation of uncertainty with a coverage factor. All measurement uncertainties are reported at the $95 \%$ confidence level except where specified otherwise.

Figure 1 schematically shows the flow loop for measuring diesel on pipe substrates. The primary components of the loop are the pump, the reservoir, and the test chamber with the test section. The inside surfaces of the approximately $96 \mathrm{~mm}$ x $1.6 \mathrm{~mm}$ rectangular flow cross-section of the aluminum test chamber, shown in Fig. 2, were black anodized to

\footnotetext{
1 "Pure diesel" is used here to denote that the particular batch of diesel, which will be consistently used throughout this project, is not mixed with water.
} 
minimize stray light reflections. The channel was designed to have the same flow area as a $13 \mathrm{~mm}$ diameter copper tube. The test chamber had a circular cavity to accept the solid pipe test section. The height of the channel was $1.6 \mathrm{~mm}$ so that the probe could be flush to the top of the test section while maintaining proximity to the test surface for measurement purposes without being an obstruction to the flow. A centrifugal pump delivered the contaminated water to the entrance of the rectangular test chamber at room temperature. The pump head was removable so that it could be easily replaced in order to test a different contaminant. The flow rate was controlled and varied by varying the pump speed with a frequency inverter. A heat exchanger immersed in the reservoir was supplied with brine from a temperature-controlled bath to maintain the entrance temperature to the test chamber at ambient temperature ( $293.8 \mathrm{~K}$ ). This was done to ensure that the diesel was at the same temperature as it was during the fluorescence calibration to avoid the temperature effect on fluorescence (Miller, 1981). An additional temperature-controlled bath was used to maintain the fluorescence standards at the same ambient temperature.

Residential copper pipe was used to plumb together the various components of the loop. Redundant volume flow rate measurements were made with an ultrasonic doppler and a turbine flowmeter with expanded uncertainties of $\pm 0.12 \mathrm{~m}^{3} / \mathrm{h}$ and $\pm 0.03 \mathrm{~m}^{3} / \mathrm{h}$, respectively. As shown in Fig. 1, three water pressure taps before and after the test chamber permit the measurement of the upstream absolute pressure and the pressure drops along the test section with expanded uncertainties of $\pm 0.24 \mathrm{kPa}$ and $\pm 1.5 \mathrm{kPa}$, respectively. Also, a sheathed thermocouple measured the water temperature at each end of the test chamber to within an uncertainty of $\pm 0.25 \mathrm{~K}$. The dissolved oxygen level, the conductivity, and the $\mathrm{pH}$, were monitored at the water reservoir with associated B-type uncertainties of $\pm 0.5 \%, \pm 50 \mu$ $(\Omega \mathrm{cm})^{-1}$, and \pm 0.3 , respectively.

Figure 1 also shows the inlet and exit taps that were used to flush the test section with fresh tap water. In preparation for flushing, the test section was isolated with valves from the rest of the test loop. Then the fluid was drained from the test chamber and returned to the reservoir. Next, a tap water supply was connected to a test chamber port. The other test chamber port was connected to a filter to absorb any diesel before it was sent to a drain.

Figure 2 shows a view of the spectrofluorometer that was used to make the fluorescence measurements and the test chamber with the fluorescence probe perpendicular to the flattened pipe test surface. Figure 3 shows a simplified schematic of the right angle spectrofluorometer consisting of a xenon light source, an excitation and an emission monochromator, and an emission photomultiplier tube (detector). The spectrofluorometer was designed to accept $45 \mathrm{~mm} \times 10 \mathrm{~mm} \times 10 \mathrm{~mm}$ fluorescent samples or cuvettes filled with fluorescent material. A special adapter with lenses and mirrors, which replaced the cuvette holder, was fabricated to remotely excite and measure fluorescence via a bifurcated optical bundle. Two optical bundles consisting of 84 fibers each originated from the spectrofluorometer. One of the bundles transmitted the excitation light, i.e., the incident intensity $\left(I_{0}\right)$, to the test pipe surface. The other bundle carried the emission, i.e, the fluorescence intensity $(F)$, from the test surface to the spectrofluorometer. The optical bundles originating from the spectrofluorometer merge transmitting and receiving fibers randomly into a single probe before entering the test section chamber. The sensor end of the 
fluorescence probe is sheathed with a quartz tube to protect it from reacting with the contaminant in the test fluid.

As the name suggests, right angle spectrometry was designed to limit the interference of the excitation signal on the emission signal by orientating the detector perpendicular to the beam of the emission monochromator. Considering this, the parallel configuration of the excitation and emission at the measuring end of the bifurcated optical bundle as shown in Fig. 2 is not ideal but was necessary for this application. The parallel configuration allows the reflection of the excitation from the copper surface to be transmitted through the emission fiber optics and to the detector. This can be a serious limitation given that the reflected excitation can overwhelm the emission signal even if the emission wavelength $\left(\lambda_{m}\right)$ and the excitation wavelength $\left(\lambda_{x}\right)$ differ because: (1) the excitation intensity can be several orders of magnitude greater than that of the fluorescence emission, and (2) the filtering process of the emission monochromator is not complete enough to entirely remove the reflected wave. The filtering process of the monochromator supplies the detector with an intensity that is distributed about the desired wavelength but with relatively small tails at larger and smaller wavelengths. Consequently, if the excitation intensity is very large, the tails of the excitation distribution can be greater than the peak emission intensity. A successful remedy for reducing the interference of the excitation signal was to place a $10 \mathrm{~nm}$ bandwidth bandpass interference filters at the exit of the excitation monochromator and one before the entrance to the excitation monochromator. Figure 3 schematically shows the placement of the bandpass interference filters.

The excitation wavelength and the emission wavelength were set to $434 \mathrm{~nm}$ and $485 \mathrm{~nm}$, respectively, for all tests. As Appendix A details, the choice of these wavelengths ensured that a significant and measurable emission signal was obtained with no measurable overlap of the excitation and emission spectra.

\section{TEST FLUIDS}

A $2 \%$ by mass diesel mixture was prepared with local Gaithersburg, MD tap water and the mixture was left to form a colloid for approximately 3 months to provide sufficient time for the diesel and the water to reach equilibrium. While the method of preparation may not reflect the most likely contamination scenario, the methodology does provide a consistent test fluid for examining the effect of flow rate on contamination because the flow rate is varied for fixed fluid properties. The measured dissolved oxygen level, the conductivity, and the $\mathrm{pH}$, of the water at $24^{\circ} \mathrm{C}$ before mixing with diesel were found to be, $86.4 \%$, $358 \mu \Omega / \mathrm{cm}$, and 7.04, respectively. Number 2 diesel fuel was used from a single batch throughout the experiment to avoid property variations that might be caused by batch variations due to it being a complex mixture of hydrocarbons. Appendix B provides the measured viscosity and density of the pure diesel liquid.

Because diesel is a complex mixture, its hydrolysis results in a dispersed phase of differing components that reside in separate regions of the colloid depending on the density, dispersion size, and hydrophobic nature of each component. If quiescent, the test reservoir had a stable Brownian suspension within the bulk water, which likely differed chemically from the dispersed phase that floated on top of the bulk liquid, and that which rested on the bottom of 
the reservoir. The result of and the evidence for a chemical breakdown of the diesel is given in Appendix C, which shows that the peak fluorescence emission for the emulsified water diesel mixture taken from the reservoir exists at a wavelength that is $25 \mathrm{~nm}$ greater than that of pure diesel. Because of the hydrolysis of diesel, positive and/or negative bias errors are likely to occur in the mass measurement depending on the individual spectra of the fluorescing components of the hydrolyzed diesel. For example, a positive bias error may result because nonfluorescent components that contributed to the diesel mass during the calibration may not deposit on the surface. Likewise, a negative bias error may occur because the peak intensity of the fluorescent material on the test surface has shifted from that of the calibration.

The hydrolysis of the diesel and the configuration of the inlet and the outlet of the reservoir influence the flow in the test section. As shown schematically in Fig.1, the opening of the pump suction line in the reservoir is situated approximately $10 \mathrm{~mm}$ below the liquid-air interface. This design entrains the hydrolyzed diesel floating on the water surface with that in the bulk water, and on the bottom of the reservoir into the pumped flow. The return flow entering the bottom of the reservoir ensured good flow mixing. Figure 4 depicts the colloidal flow within the test section and the fluorescent measurement probe above it for the contamination and decontamination test conditions. The size of the droplets in the dispersed flow is exaggerated for illustration purposes. Both test conditions are shown to have an excess layer thickness $\left(l_{\mathrm{e}}\right)$ of undiluted hydrolyzed diesel adsorbed to the test surface. Because the molar mass of the diesel is unknown, the surface excess density $(I)$ is defined in the work on a mass basis as (Kedzierski, 2001):

$$
\Gamma=l_{\mathrm{e}}\left(\rho_{\mathrm{d}}-\rho_{\mathrm{b}} x_{\mathrm{b}}\right)
$$

The density of liquid diesel is $\rho_{\mathrm{d}}$. The density of the bulk mixture $\rho_{\mathrm{b}}$ is evaluated at the bulk mass fraction of the mixture $\left(x_{\mathrm{b}}\right)$. The surface excess density is roughly the mass of diesel attached per surface area. The $\Gamma$ and $l_{\mathrm{e}}$ are the primary measurements of this study.

\section{MEASUREMENTS AND UNCERTAINTIES}

Fluorescence/Mass Calibration

Fluorescence as a means for detecting a contaminant has its advantages in that its absorption and fluorescence spectra are like a fingerprint that can be used in its identification.

Consequently, by isolating the wavelength of light that the contaminant fluoresces, its intensity can be used to identify its mass. This is true even when the contaminant is mixed with another fluorescent or nonfluorescent substance as long as the fluorescent substance does not absorb and emit at the same wavelengths as the contaminant. For this reason, the tap water was examined and it was not found to fluoresce at any wavelength for any excitation wavelengths between the range or $200 \mathrm{~nm}$ and $800 \mathrm{~nm}$. Consequently, interference from water is not possible via it contributing to the intensity of the fluorescence signal.

The calibration technique that was developed here for detecting the mass of diesel on a copper surface exposed to a flowing dilute mixture of diesel in water is introduced in the following. Two different calibration methods had to be combined due to the additional 
complexity caused by immiscible liquids. Both calibration techniques were used to quantify different functional aspects of the Beer-Lambert-Bougher law (Amadeo et al., 1971), which forms the basis of the calibration equation. The first method is essentially the same as the original NIST calibration method that was used to detect lubricants on boiling surfaces (Kedzierski, 2002). This methodology was used to obtain the relationship between diesel composition and fluorescence intensity for a fixed light path length (fixed probe height above the test surface). ${ }^{2}$ The second method, that was developed in this study, relies on a perpendicular traverse of the flow stream with the measurement probe. To achieve this, a linear positioning device with a graduated knob was adapted to the quartz tube as shown in Fig. 2. The second method (traverse method) is used to calibrate the effect of contaminant thickness (path length) and the proximity of incident intensity. The traverse method is essential for splitting the total measured fluorescent intensity into two components: that from the diesel on the test surface and that from the diesel in the bulk flow stream. In this way, the mass of diesel on the test surface and the composition of the fluid stream are determined.

Figure 5 shows the vessel that was used in the first method to calibrate the fluorescence intensity received from the bifurcated optical bundle against the mass fraction of diesel. The lid of the $150 \mathrm{~mL}$ black, anodized, metal jar had a port for evacuation ${ }^{3}$ and filling of the test sample and a fitting to seal around the stainless tube that pierced the lid. The stainless tube had a quartz tube and a quartz bottom welded to its end and it was the same type that was used in the test chamber of Fig. 2. A disk of copper pipe material was placed on the bottom of the jar. By using the same material and surface roughness, the disk and the test pipe had the same reflective properties. Copper from a flattened pipe was evenly oxidized by electrolysis and soldered to the top of the calibration disk that had circumferentially machined grooves for sealing in the test chamber. The same disk was used as the calibration disk and the test surface to compensate for unknown surface effects. The distance between the top of the calibration disk and the bottom of the quartz tube was set with the aid of a 1.6 mm Teflon ${ }^{4}$ gauge disk and micrometer dial indicators. This fixes the path length of the fluorescence and the mass of fluorescent liquid below the probe. During calibration, the jar and the portion of the quartz tube above the lid were covered with black insulation to prevent the optical probe from receiving ambient light. The probe rested on the inside-bottom of the quartz tube.

Three jars were used to calibrate the mass fraction of diesel to the fluorescent intensity. Two jars were used as standards to set the lower (0) and upper (100) limits of the intensity signal on the spectrofluorometer. A jar that contained only pure water was used to zero the intensity. Because light intensities are additive, the zeroing ensured that the reflected

\footnotetext{
${ }^{2}$ The first method would have been sufficient had the bulk composition of the flow remained the same as it was charged in the reservoir. Due to the immiscibility of the two fluids, the bulk composition of the flow differs from that in the reservoir.

${ }^{3}$ It has been found that weak evacuation of a vessel containing diesel does not measurably change its fluorescent characteristics.

${ }^{4}$ Certain trade names and company products are mentioned in the text or identified in an illustration in order to adequately specify the experimental procedure and equipment used. In no case does such an identification imply recommendation or endorsement by the National Institute of Standards and Technology, nor does it imply that the products are necessarily the best available for the purpose.
} 
excitation wave and other effects were not attributed to fluorescence. A second jar that contained pure diesel was used to set the intensity on the spectrofluorometer to 100 . The third jar was used to measure and record the intensity of various mixtures of diesel and nonfluorescent $n$-decane of different concentrations. N-decane was used instead of water because it was miscible with diesel and also non-fluorescent. As an additional precaution, all raw-measured intensities $\left(F_{\mathrm{r}}\right)$ were numerically normalized by the intensity from the zero-jar $\left(F_{0}\right)$ and the maximum-jar $\left(F_{100}\right)$ :

$$
F=\frac{F_{\mathrm{r}}-F_{0}}{F_{100}-F_{0}}
$$

where the intensity of the contamination data was adjusted (see Appendix D) by no more than $0.3 \%$ to account for the small (typically within $\pm 1 \mathrm{~K}$ ) difference in temperature between the test section and the bath containing the maximum- and the zero-jars. The maximum correction for the flushing data was greater $(1.5 \%)$ than for the contamination measurements due to the colder temperature of the house tap water.

Evacuation of the jar was done to prevent fluorescence quenching by oxygen (Guilbault, 1967). N-decane was used because it is miscible with diesel. Calibration measurements proceed by successively adding or removing diesel in appropriately small increments. As shown in Appendix E, the fluorescence intensity was fitted linearly with respect to the diesel mass fraction to within a residual standard deviation of $\pm 1.2 \%$.

The second calibration method involved pure diesel alone and varying the thickness of the diesel below the quartz probe to determine the effect of the proximity of the incident light $\left(I_{o}\right)$ and its path length $(l)$. For these tests, the probe was traversed through the diesel and diesel thickness below the quartz probe was synonymous with the path length. As shown in Fig. 2, a linear positioning device with a graduated knob was used to locate the quartz tube relative to the test surface and thus measure the path length of the incident light through the diesel. The measured fluorescent intensity versus the path length was non-linear as shown in Appendix E. Given that the intensity versus mass fraction followed a linear relationship, the nonlinear aspect of the intensity versus $l$ was due to the variation in the incident intensity with $l$. For this reason, further calibrations were done with fixed diesel film thickness and variable path lengths and it was observed that $\frac{1}{F} \frac{d F}{d l}$ was approximately constant for all ranges of the $F$ and $l$ traverse data for fixed diesel film thickness. This demonstrates the exponential dependence of $I_{o}$ with the proximity of the probe to the diesel $(l)$ and that this was the cause of the nonlinear calibration with respect to $l$. The $I_{o}$ path length effect is known as excitation absorbance (Herman, 1998), which results from the diesel nearest to the light source receiving more excitation than the diesel that is further away.

The linear form of the Beer-Lambert-Bougher law (Amadeo et al., 1971) was used to correlate the measured intensity of the fluorescence emission $(F)$ to the mass of diesel:

$$
F=2.3 I_{o} \varepsilon c l \Phi \rightarrow[\varepsilon c l \leq 0.05]
$$


Here $c$ is the concentration of the fluorescent diesel, which can be rewritten as a product of the bulk contaminant (diesel) mass fraction $\left(x_{\mathrm{b}}\right)$ and the bulk liquid mixture density $\left(\rho_{\mathrm{b}}\right)$ divided by the molar mass of the contaminant $\left(M_{\mathrm{c}}\right)$. Appendix F shows that the linear criteria for eq. (3) ( $\varepsilon c l \leq 0.05$ ) is satisfied for $78 \%$ of the calibration data and the absorbance ( $\varepsilon c l$ ) did not exceed 0.063. In addition, the use of the full, nonlinear Beer-Lambert-Bougher law did not reduce the residual standard deviation of the fit. Consequently, use of the linear form of the law is justified.

The mixture densities were calculated on a linear mass weighted basis. The quantum efficiency of the fluorescence $(\Phi)$, the extinction coefficient $(\varepsilon)$, the intensity of the incident radiation $\left(I_{o}\right)$, and the $M_{\mathrm{c}}$ are all unknowns that are lumped into two regression constants and an exponential term to give the regressed calibration of $F$ against $x_{\mathrm{b}}$ for diesel as:

$$
F=\frac{2.3 I_{o} \Phi \varepsilon}{M_{\mathrm{c}}} l x_{\mathrm{b}} \rho_{\mathrm{b}}=1.04735\left[\frac{\mathrm{m}^{2}}{\mathrm{~kg}}\right] l x_{\mathrm{b}} \rho_{\mathrm{b}} e^{-209.23 \mathrm{~m}^{-1} l}
$$

Equation (4) shows that $2.3 I_{o} \Phi \varepsilon M_{\mathrm{c}}^{-1}=1.04735\left[\mathrm{~m}^{2} \mathrm{~kg}^{-1}\right] e^{-209.23 \mathrm{~m}^{-1} l}$. The uncertainty of the calibration given in eq. (4) is approximately $\pm 0.2 \%$ of $F$ for the $95 \%$ confidence level.

Figure 6 shows that the resulting calibration for the flow conditions is linear. The regression of the same measurements against the Beer-Lambert-Bougher law (Amadeo et al., 1971) gave a greater fit uncertainty suggesting that the linear fit is more appropriate.

\section{Application of Calibration}

Given that $\Gamma$ and $l_{\mathrm{e}}$ are the primary measurements of this study, the main use of the calibration is to solve for these parameters. For the case where the diesel remains completely immiscible with water and has a strong affinity for metal surfaces, an excess layer of pure diesel will form on the pipe surface of thickness $l_{\mathrm{e}}$.

Equation 3 can be rearranged to solve for the diesel excess layer thickness by setting the mass fraction, and the mixture density to reflect the properties of pure diesel:

$$
l_{\mathrm{e}}=\frac{F}{2.3 I_{\mathrm{o}} \Phi \varepsilon M_{\mathrm{c}}^{-1} \rho_{\mathrm{d}}}=A_{0}+A_{1} l
$$

As shown in Fig. 7, $l_{\mathrm{e}}$ can be regressed to eq. (5) using measurements of $F$ for given values of path length $(l)$ and plotted versus $l$. The two example $F$ versus $l$ data sets shown in Fig. 7 were obtained by moving the optical bundle closer to the test surface in order to vary the path length. As illustrated by the open circles, most of the resulting values of $l_{\mathrm{e}}$ for a given data set were directly proportional to $l$; hence, a linear relationship with respect to $l$ including fitting constants $A_{0}$ and $A_{1}$ is shown on the rightmost side of eq. (5). Although, eq. (5) can be used to calculate as many values of $l_{\mathrm{e}}$ as long values of $F$ and $l$ can be supplied, it is valid only for when the path length and the excess layer thickness coincide (for non-zero bulk 
compositions) because it has been derived for pure diesel. This condition can be met by setting $l$ to $l_{\mathrm{e}}$ in the rightmost portion of the eq. (5) and solving for $l_{\mathrm{e}}$ :

$$
l_{\mathrm{e}}=\frac{A_{0}}{1-A_{1}}
$$

For traverse data sets that are not linear for the full range of $l$, as illustrated by the open square symbols in Fig. 7, only the data that is approximately linear near the wall was used to generate constants $A_{0}$ and $A_{1}$.

Equation 6 is necessary only for a non-zero bulk mass fraction $\left(x_{\mathrm{b}}\right)$. For flushing tests, where $x_{\mathrm{b}}=0$, eq. (5) is valid for all $l \geq l_{\mathrm{e}}$. Consequently, the excess surface density of diesel for flushing tests is obtained from an average of the $l_{\mathrm{e}}$ obtained from eq. (5) and the traverse measurements.

As shown in Appendix G, roughly $85 \%$ of the $l_{\mathrm{e}}$ measurements have a relative uncertainty of less than $25 \%$ for the $95 \%$ confidence level. For these measurements the average uncertainty of $l_{\mathrm{e}}$ is approximately $\pm 7 \%$ of $l_{\mathrm{e}}$. Overall, the average uncertainty of $l_{\mathrm{e}}$ was approximately $\pm 0.2 \mu \mathrm{m}$.

The bulk mass fraction can be obtained by dividing the total fluorescence signal $(F)$ into its components along the path length while assuming a uniform bulk mass fraction. The total intensity is the sum of that contributed by the bulk concentration $\left(F_{l}\left(x_{\mathrm{m}}=x_{\mathrm{b}}\right)\right)$ for the entire path length and that in the diesel excess layer $\left(F_{l \mathrm{e}}\left(x_{\mathrm{m}}=1\right)\right)$ minus the intensity that would have been due to the bulk concentration but did not occur because it was displaced by the excess layer $\left(F_{l e}\left(x_{\mathrm{m}}=x_{\mathrm{b}}\right)\right)$

$$
F=F_{l}\left(x_{m}=x_{b}\right)-F_{l_{\mathrm{e}}}\left(x_{m}=x_{b}\right)+F_{l_{\mathrm{e}}}\left(x_{m}=1\right)
$$

Substitution of eq. (4) into the components of the above equation and grouping like terms gives:

$$
F=2.3 I_{o} \Phi \varepsilon M_{\mathrm{c}}^{-1}\left[l x_{b} \rho_{b}-l_{e} x_{b} \rho_{b}+l_{e} \rho_{d}\right]
$$

Here $\rho_{\mathrm{d}}$ is the density of liquid diesel.

When the expression for the linearly mass fraction weighted $\rho_{\mathrm{b}}$ is subsitituted into eq. (8), its solution is quadratic in $x_{\mathrm{b}}$ with only one root that is less than or equal to 1 .

$$
x_{\mathrm{b}}=\frac{1}{2}\left(\frac{1}{1-\frac{\rho_{\mathrm{d}}}{\rho_{\mathrm{w}}}}\right)-\frac{1}{2} \sqrt{\left(\frac{1}{1-\frac{\rho_{\mathrm{d}}}{\rho_{\mathrm{w}}}}\right)^{2}-\left(\frac{4\left(\frac{F}{2.3 I_{o} \Phi \varepsilon M_{\mathrm{c}}^{-1}}-l_{e} \rho_{d}\right)}{\left(l-l_{\mathrm{e}}\right)\left(\rho_{\mathrm{w}}-\rho_{\mathrm{d}}\right)}\right)}
$$


where $\rho_{\mathrm{w}}$ is the density of liquid tap water. The average uncertainty of $x_{\mathrm{b}}$ was approximately \pm 0.002 .

Air Gap Calibration

A secondary methodology was developed that relies on the gradient of $F$ rather than its absolute value in order to confirm the measurement of $l_{\mathrm{e}}$ as obtained from eq. (5) or eq. (6). The advantage of a gradient approach would be the elimination of a bias error on the measurement of $F$ if it existed. As shown in Fig. 4, part of the excitation is reflected from the diesel-air interface and is not available to enduce flourescence. Consequently, the calibration must be adjusted to account for the air gap during the drained test chamber measurements. Appendix E provides the derivation of the air-gap $l_{\mathrm{e}}$ and the result is given here as:

$$
l_{\mathrm{e}}=\frac{-0.0121 \mathrm{~m} \frac{d F_{\mathrm{ag}}}{d l} M_{\mathrm{c}}}{2.3 I_{o} \Phi \varepsilon x_{\mathrm{m}} \rho_{\mathrm{m}}}=-0.01156 \frac{\mathrm{kg}}{\mathrm{m}} \frac{\frac{d F_{\mathrm{ag}}}{d l} e^{209.23 \mathrm{~m}^{-1} l}}{x_{\mathrm{m}} \rho_{\mathrm{m}}}
$$

\section{MEASUREMENT RESULTS}

\section{Excess Layer Thickness}

The test apparatus shown in Fig. 1 was used to submit an oxidized copper disk to exposure tests with two different bulk concentrations of diesel in tap water under varying flow conditions. More specifically, contamination measurements over an approximate $200 \mathrm{~h}$ time period were made for five different Reynolds numbers varying from 0 to 7000:

$$
\operatorname{Re}=\frac{4 \dot{m}}{\mu_{\mathrm{b}} p_{w}}
$$

where the wetted perimeter of the channel was $195 \mathrm{~mm}$, the viscosity of the mixed bulk flow $\left(\mu_{\mathrm{b}}\right)$ was calculated using a nonlinear mixture equation, and the mass flow rate $(\dot{m})$ was obtained from the turbine meter. Flushing measurements were done for a fixed Re of approximately 5000. The range of Reynolds numbers result from using a range of volume flow rates that a half-inch diameter tube would experience in typical buildings. After each contamination tests, the test surface was cleaned with acetone and clean tap water. Appendix $\mathrm{H}$ provides tabulated measurements for both the raw and reduced data.

Figures 8 provides the measured diesel layer thickness as caused by an exposure to a flowing water/diesel (99.8/0.2) mixture, i.e., diesel at approximately $0.2 \%$ bulk mass fraction (2000 ppm). The exposure time is the duration of exposure of the test surface to the flow starting from when the clean surface was first exposed to a particular flow condition. For all flow rates and exposure times, the average $l_{\mathrm{e}}$ for $x_{\mathrm{b}}=0.2 \%$ obtained from the eq. (6) methodology was approximately $2.3 \mu \mathrm{m}$. 
Figures 9 provides the measured diesel layer thickness as caused by an exposure to a flowing water/diesel (99.7/0.3) mixture, i.e., diesel at approximately $0.3 \%$ bulk mass fraction (3000 ppm). A much larger variability in the measurements is evident for the $0.3 \%$ mass fraction than for the $0.2 \%$ mass fraction condition. For all exposure times and Re, the average $l_{\mathrm{e}}$ for $x_{\mathrm{b}}=0.3 \%$ was approximately $7.4 \mu \mathrm{m}$, which is $5.1 \mu \mathrm{m}(222 \%)$ thicker than the average thickness observed for the $0.2 \%$ mass fraction tests.

Figure 10 crossplots all of the excess layer measurements of Fig. 8 as a function of Re. Figure 10 shows that the maximum diesel excess layer thickness of approximately $8 \mu \mathrm{m}$ occurred at a Re near 4800. For Re larger and smaller than 4800, the diesel excess layer was thinner. For example, the $l_{\mathrm{e}}$ for Re near 1900 and 3800 was approximately $1 \mu \mathrm{m}$, which is nearly eight times less than the maximum $l_{\mathrm{e}}$. The $l_{\mathrm{e}}$ for Re greater than 6000 was approximately $3 \mu \mathrm{m}$. Figure 11 crossplots all of the excess layer measurements of Fig. 9 (the $0.3 \%$ mass fraction tests) as a function of Re. Figure 11 shows that the maximum film thickness of approximately $26 \mathrm{~nm}$ occurred at a Re of approximately 4000. Consequently, a maximum for the diesel adsorption exists near a Re of 4000 for both freestream concentrations. The dashed lines given in Figs. 10 and 11 represent the maximum measured excess layer for each range of Re tests. The variation in Re for a given set of tests for "fixed" Re was caused by an approximate $1 \%$ variation in the water temperature during startup and the an approximate $15 \%$ variation in the water flow during the nearly $200 \mathrm{~h}$ test duration.

Filled symbols in Figs. 8 and 10, shown between $150 \mathrm{~h}$ and $200 \mathrm{~h}$, represent $l_{\mathrm{e}}$ measurements that were made at the end of the exposure tests after the test section was drained using the air-gap technique as a secondary measurement technique. For the water/diesel (99.8/0.2), mixture all three of the drain checks were within $\pm 1.5 \mu \mathrm{m}$ of the measurements that were made while the test fluid was flowing. For example, for the air-gap check obtained using eq. (10) for the Re near 7000 condition produced a $l_{\mathrm{e}}$ near $3 \mu \mathrm{m}$, while the last measurement made with eq. (6) produced a $l_{\mathrm{e}}$ near $3.7 \mu \mathrm{m}$. Similarly, eq. (6) produced a $l_{\mathrm{e}}$ of approximately $2 \mu \mathrm{m}$ for both the no-flow and the 3200-Re tests, while the eq. (10) check resulted in $3.5 \mu \mathrm{m}$ and $1 \mu \mathrm{m}$ for $l_{\mathrm{e}}$, respectively. For the water/diesel (99.7/0.3) mixture, all three of the drain checks for the flushing data were also within $\pm 1.5 \mu \mathrm{m}$ of the measurements that were made while the test fluid was flowing giving $0.5 \mu \mathrm{m}$ and $-0.5 \mu \mathrm{m}$, respectively. However, the agreement between the empty and filled test chamber tests was not as good for the water/diesel (99.7/0.3) mixture for the 7000-Re contamination measurements. For example, the air-gap check for the Re near 7000 condition produced a $l_{\mathrm{e}}$ near $5 \mu \mathrm{m}$, while the last measurement made with eq. (4) produced a $l_{\mathrm{e}}$ near $2 \mu \mathrm{m}$.

Flushing tests done after the water/diesel (99.8/0.2) 4800-Re contamination tests are shown in Fig. 8. The flushing measurements start at an $l_{\mathrm{e}}$ near $6.5 \mu \mathrm{m}$, which agrees with the value of $l_{\mathrm{e}}$ at the end of the 4600-Re contamination tests, thus, confirming the repeatability of the measurement technique. The $l_{\mathrm{e}}$ decreased from approximately $6.5 \mu \mathrm{m}$ to approximately $1.5 \mu \mathrm{m}$ after flushing for approximately $55 \mathrm{~h}$. This corresponds roughly to a $0.09 \mu \mathrm{m} / \mathrm{h}$ removal rate and a $77 \%$ reduction of the total diesel thickness over $55 \mathrm{~h}$. 
The flushing tests shown in Fig. 10 performed after the 5000-Re water/diesel (99.7/0.3) contamination tests, likewise start at approximately the same $l_{\mathrm{e}}(1.5 \mu \mathrm{m})$ as where the previous contamination test ended, again demonstrating good repeatability. After approximately $20 \mathrm{~h}$ of flushing, the 5000-Re contaminant thickness was reduced to approximately $-0.5 \mathrm{~nm}$. Given the uncertainty of the measurement, most all of the diesel has been removed by flushing with clean tap water. The removal rate achieved after the 5000Re, $0.3 \%$ mass fraction $(3000 \mathrm{ppm})$ contamination tests $(0.1 \mu \mathrm{m} / \mathrm{h})$ agrees closely with that achieved for the flushing tests done after the 4600-Re, $0.2 \%$ mass fraction (2000 ppm) contamination tests. This suggests a constant removal rate of approximately $0.1 \mu \mathrm{m} / \mathrm{h}$ of diesel from a copper surface for a flushing Re of 5000 that is independent of initial thickness and original contamination concentration. No removal rate could be calculated for the flushing tests done after the 7000-Re water/diesel (99.7/0.3) because the tests produced an $l_{\mathrm{e}}$ near $-0.5 \mathrm{~nm}$ for nearly all measurement times.

\section{Freundlich Constants}

For sorption systems, the Freundlich constant $(K)$ relates the equilibrium solid-phase concentration $\left(c_{\mathrm{s}}\right)$ to the equilibrium concentration of the bulk liquid-phase $\left(c_{1}\right)$ as (Schwarzenbach et al., 2003):

$$
c_{\mathrm{s}}=K c_{1}^{\mathrm{n}}
$$

where the Freundlich exponent (n) determines the rate of sorption to the solid surface.

Equation 12 can be rewritten in terms of the excess surface density and the mass fraction of the bulk liquid as:

$$
\Gamma=\frac{K}{a_{\mathrm{s}}}\left(\frac{x_{\mathrm{b}} \rho_{\mathrm{b}}}{M_{\mathrm{c}}}\right)^{\mathrm{n}}=\hat{K}\left(x_{\mathrm{b}} \rho_{\mathrm{b}}\right)^{\mathrm{n}}
$$

where the constant $a_{\mathrm{s}}$ is the specific surface area of the solid defined as the surface area per mass of solid. Here $K$ is normalized by the constant $a_{\mathrm{s}}$ and the molar mass of the contaminant raised to the $\mathrm{n}^{-1}$ power $\left(M_{\mathrm{c}}^{\mathrm{n}^{-1}}\right)$ to give $\hat{K}$.

Because the present measurements do not sufficiently span the free stream concentration variable to accurately determine the Freundlich exponent, it was assumed that the dieselwater-copper system behaved as one with constant sorption free energies giving a linear isotherm with $n=1$. Using this assumption, the normalized "Freundlich constants" were obtained by averaging measurements for a particular Re test for exposure times greater than $50 \mathrm{~h}$ in order to approach an equilibrium or steady state value for $\hat{K}$. All of the $l_{\mathrm{e}}$ measurements, for a given Re test, appeared to be nearly constant after $50 \mathrm{~h}$ of exposure. Consequently, it was assumed that a balance between diesel deposition and removal had been achieved. 
Figure 12 shows the normalized Freundlich constant as a function of Re for the two different bulk concentrations of this study. The figure shows that $\hat{K}$ varies between near zero to values approaching $0.015 \mathrm{~m}$. For Re less than 4000, the $\hat{K}$ behaves as expected with values for the $0.3 \%$ bulk mass fraction being larger than those for the $0.2 \%$ bulk mass fraction. However, for Re greater than 4000, the opposite behavior is observed, with $\hat{K}$ 's for the $0.2 \%$ mass fraction being larger than those for the $0.3 \%$ mass fraction. Considering that a Re of 4000 is beyond the transition Re (from laminar to turbulent flow) ${ }^{5}$ and within the transition region, no explanation can be offered at this time for the crossover phenomenon.

\section{DISCUSSION}

Because of its derivation from thermodynamics, the Freundlich constant given in eq. (12) is associated with chemical and/or physical equilibrium between the liquid-phase and the solidphase concentrations. The measured phenomenon of the present study cannot be expressed in terms of a solid-phase concentration. The solid is not soluble with respect to the contaminant. Rather, the contaminant is located at the solid surface. As a result, the normalized "Freundlich constant" given by eq. (13) $(\hat{K})$ may not necessarily represent thermodynamic equilibrium. The $\hat{K}$ may be influenced by physical forces other than Van der Waals like flow and pressure forces. For this reason, the variation of the normalized Freundlich constant with respect to Re for fixed liquid-phase concentration is not prohibited by thermodynamics.

It is difficult to estimate the effect of the hydrolyzed diesel on the measurements, but it has likely introduced an unknown bias error to measurements. Future test with fresh diesel and water mixtures that have not had time to hydrolyze would reduce or eliminate the bias error.

\section{CONCLUSIONS}

A detailed account of the development of a fluorescence based measurement technique for measuring the mass of contaminant on solid surfaces in the presence of water flow has been provided. A test apparatus was designed and developed to use the fluorescent properties of diesel for the purpose of studying its adsorption and desorption to and from an oxidized copper test surface. A calibration technique was developed to measure both the mass of diesel adsorbed per unit surface area (the excess surface density) and the bulk concentration of the hydrolyzed diesel in the flow.

The measured diesel excess surface density that was adsorbed to the surface varied between zero and $0.02 \mathrm{~kg} / \mathrm{m}^{2}$ for Reynolds numbers between zero and 7000 . A maximum for the diesel adsorption was observed near a Re of 4000 for both nominal bulk mass fractions of $0.2 \%$ and $0.3 \%$ diesel. For the most part, the thickness of the diesel excess surface density remained less than $10 \mu \mathrm{m}$. The exception to these excess layer measurements was the $0.3 \%$ bulk mass fraction with $\mathrm{Re}=4000$ measurements that peaked near $25 \mu \mathrm{m}$.

In an effort to model the adsorption of diesel to copper, normalized Freundlich constants were calculated based on a linear isotherm and found to vary between near zero and $0.015 \mathrm{~m}$.

\footnotetext{
${ }^{5}$ The transition Re would be 2300 if the hydraulic diameter concept prevails, and 3000 (Schlichting, 1979) if the flow is considered to be one between infinite flat plates.
} 
Most of the Freundlich constants were less than $0.005 \mathrm{~m}$. In addition, flushing tests suggest a constant removal rate of approximately $0.1 \mu \mathrm{m} / \mathrm{h}$ of diesel from a copper surface that is independent of initial thickness and original contamination concentration. The measurements show that most all of the diesel has been removed to within the uncertainty of the measurement procedure by flushing with clean tap water.

\section{ACKNOWLEDGEMENTS}

This work was funded by the U.S. Environmental Protection Agency (EPA) under contract \#DW-13-92167701-0, with the guidance of project manager Mr. V. Gallardo. Thanks go to the following NIST personnel for their constructive criticism of the first draft of the manuscript: Dr. S. Treado, and Dr. P. Domanski. Thanks goes to the Mr. V. Gallardo of EPA for his constructive criticism of the second draft of the manuscript. Furthermore, the author extends appreciation to Mr. D. Wilmering for taking the measurements and conquering the difficult machining and design problems encountered in the project. 


\section{NOMENCLATURE}

\section{English Symbols}
A regression constants in eq. (5)
$a_{\mathrm{s}} \quad$ specific surface area, $\mathrm{m}^{2} \mathrm{~kg}^{-1}$
c concentration, $\mathrm{mol} \mathrm{m}^{-3}$
$F \quad$ fluorescence intensity
$F_{\mathrm{r}} \quad$ raw fluorescence intensity measurement
$I_{0} \quad$ incident intensity, $\mathrm{V}$
$K \quad$ Freundlich constant, $\mathrm{mol} \cdot \mathrm{kg}^{-1}$
$\hat{K} \quad$ normalized Freundlich constant, $m$
$l$ path length, $\mathrm{m}$
$l_{e} \quad$ thickness of excess layer, $\mathrm{m}$
$M_{\mathrm{c}} \quad$ molar mass of contaminant, $\mathrm{kg} \mathrm{mol}^{-1}$
$\dot{m} \quad$ mass flow rate, $\mathrm{kg} \mathrm{s}^{-1}$
Re Reynolds number
n $\quad$ Freundlich exponent
$p_{\mathrm{w}} \quad$ wetted perimeter of channel, $\mathrm{m}$
$T$ temperature, $\mathrm{K}$
$U$ expanded uncertainty
$u_{\mathrm{i}} \quad$ standard uncertainty
$x \quad$ mass fraction of diesel

\section{Greek symbols}

$\beta \quad$ coefficient of temperature dependence, $\mathrm{K}^{-1}$

$\Gamma \quad$ surface excess density, $\mathrm{kg} \mathrm{m}^{-2}$

$\varepsilon \quad$ extinction coefficient

$\lambda$ wavelength, $m$

$\mu \quad$ dynamic viscosity, $\mathrm{kg} \mathrm{m}^{-1} \mathrm{~s}^{-1}$

$v \quad$ kinematic viscosity, $\mathrm{m}^{2} \mathrm{~s}^{-1}$

$\rho \quad$ mass density of liquid, $\mathrm{kg} \mathrm{m}^{-3}$

$\Phi \quad$ quantum efficiency of fluorescence

\section{English Subscripts}

\begin{tabular}{ll}
\hline 0 & zero reference jar \\
100 & maximum reference jar \\
a & ambient \\
ag & air gap \\
$\mathrm{b}$ & bulk \\
d & pure diesel \\
$\mathrm{e}$ & excess layer \\
$\mathrm{i}$ & inlet \\
$\mathrm{l}$ & liquid \\
$l_{\mathrm{e}}$ & excess layer \\
$\mathrm{m}$ & emission, mixture \\
$\mathrm{ng}$ & no air gap \\
$\mathrm{o}$ & outlet or exit
\end{tabular}


$\mathrm{s} \quad$ solid surface

$\mathrm{T}_{\mathrm{b}} \quad$ reference bath temperature

$\mathrm{T}_{\mathrm{T}}$ test section temperature

$\mathrm{x}$ excitation

w tap water

\section{$\underline{\text { Superscripts }}$}

- $\quad$ average 


\section{REFERENCES}

Amadeo, J. P., Rosén C., and Pasby, T. L., 1971, Fluorescence Spectroscopy An Introduction for Biology and Medicine, Marcel Dekker, Inc., New York, p. 153.

Guilbault, G. G., 1967, Fluorescence: Theory, Instrumentation, and Practice, Edward Arnold LTD., London, pp. 91-95.

Herman, B., 1998, Fluorescence Microscopy, $2^{\text {nd }}$ ed., Springer-Verlag New York, Inc., pp. $69-71$.

Kedzierski, M. A., 2003, ”Effect of Bulk Lubricant Concentration on the Excess Surface Density During R134a Pool Boiling with Extensive Measurement and Analysis Details,” NISTIR 7051, U.S. Department of Commerce, Washington, D.C.

Kedzierski, M. A., 2002, ”Use of Fluorescence to Measure the Lubricant Excess Surface Density During Pool Boiling,” Int. J. Refrigeration, Vol. 25, pp.1110-1122.

Kedzierski, M. A., 2001, ”Use of Fluorescence to Measure the Lubricant Excess Surface Density During Pool Boiling,” NISTIR 6727, U.S. Department of Commerce, Washington, D.C.

Miller, J. N., 1981, Volume Two Standards in Fluorescence Spectrometry, Chapman and Hall, London, pp. 44-67.

Reader, J, Corliss, C. H., Wiese, W. L., and Martin, G. A., 1980, "Wavelengths and Transition Probabilities of Atoms and Atomic Ions", NSRDS-National Bureau of Standards \#68, U.S. Department of Commerce, Washington.

Schlichting, H., 1979, Boundary-Layer Theory, McGraw-Hill, New York, pg. 591.

Schwarzenbach, R. P., Gschwend, P. M., and Imboden, D., M., 2003, Environmental Organic Chemistry, $2^{\text {nd }}$ ed., Wiley, NJ, pp 281-283.

Simplex, 2006, http://www.simplexdirect.com/FuelSupply/mainten04.html 


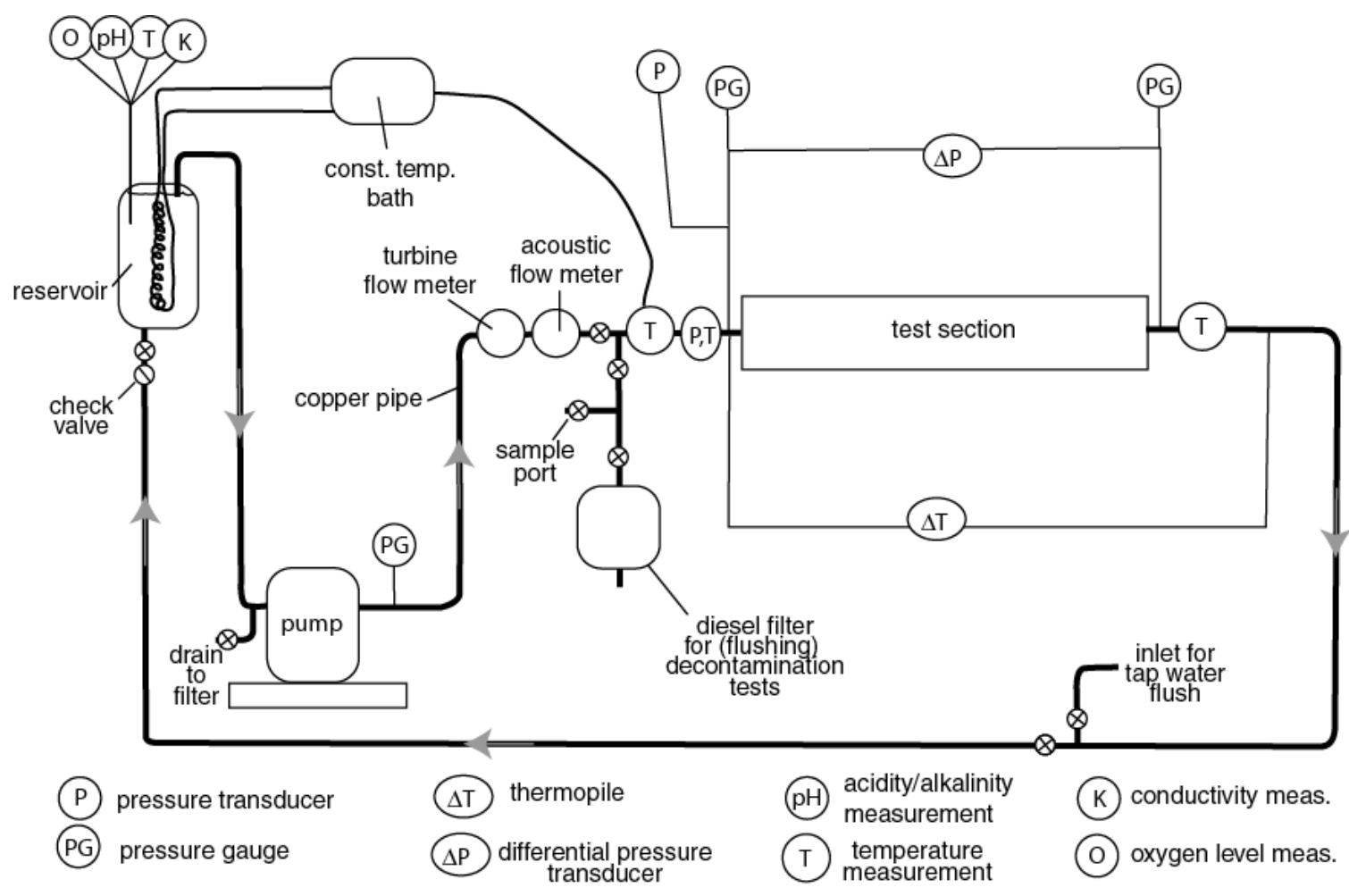

Fig. 1 Schematic of test loop 


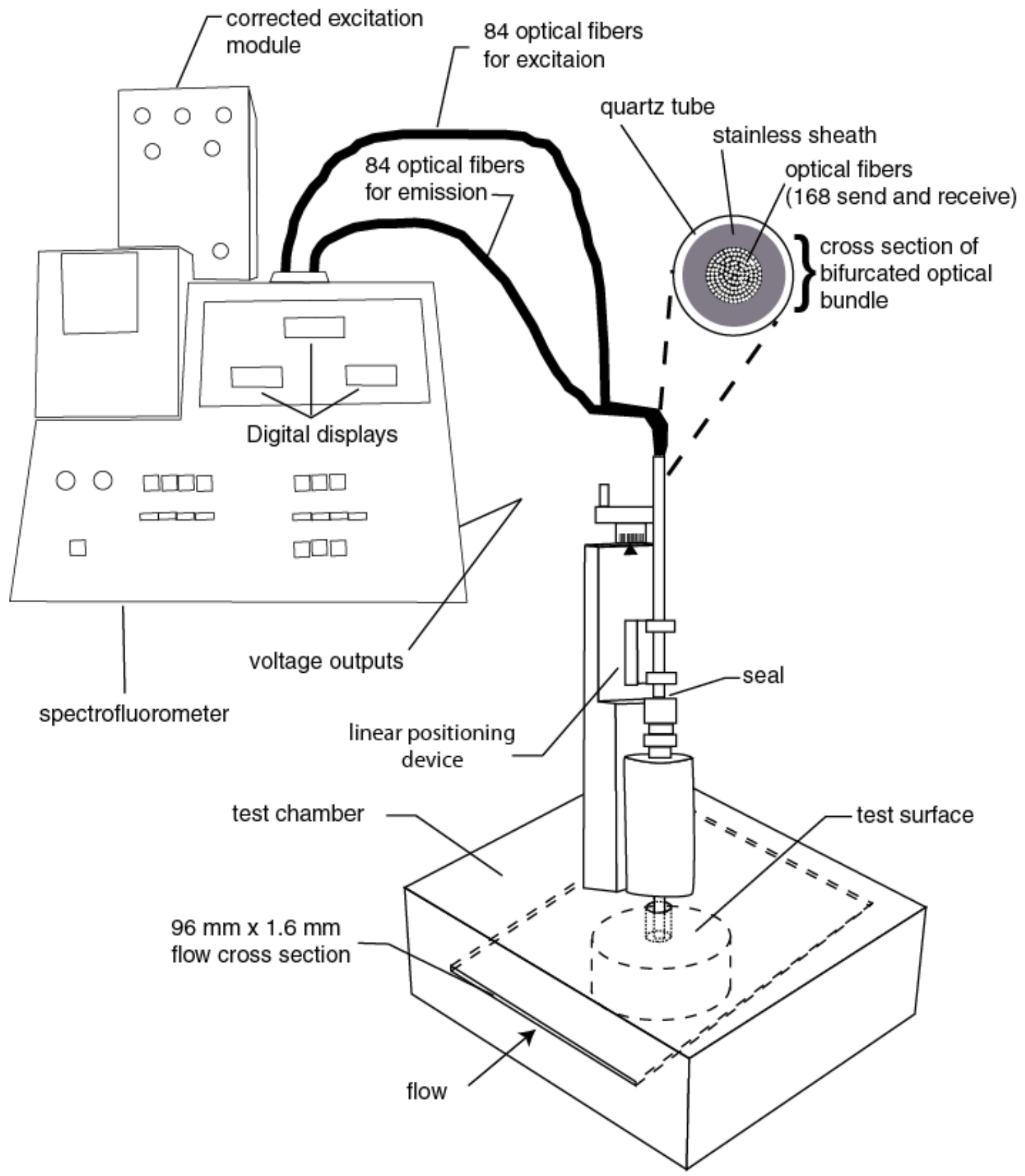

Fig. 2 Schematic of spectrofluorometer, test section, and linear positioning device 


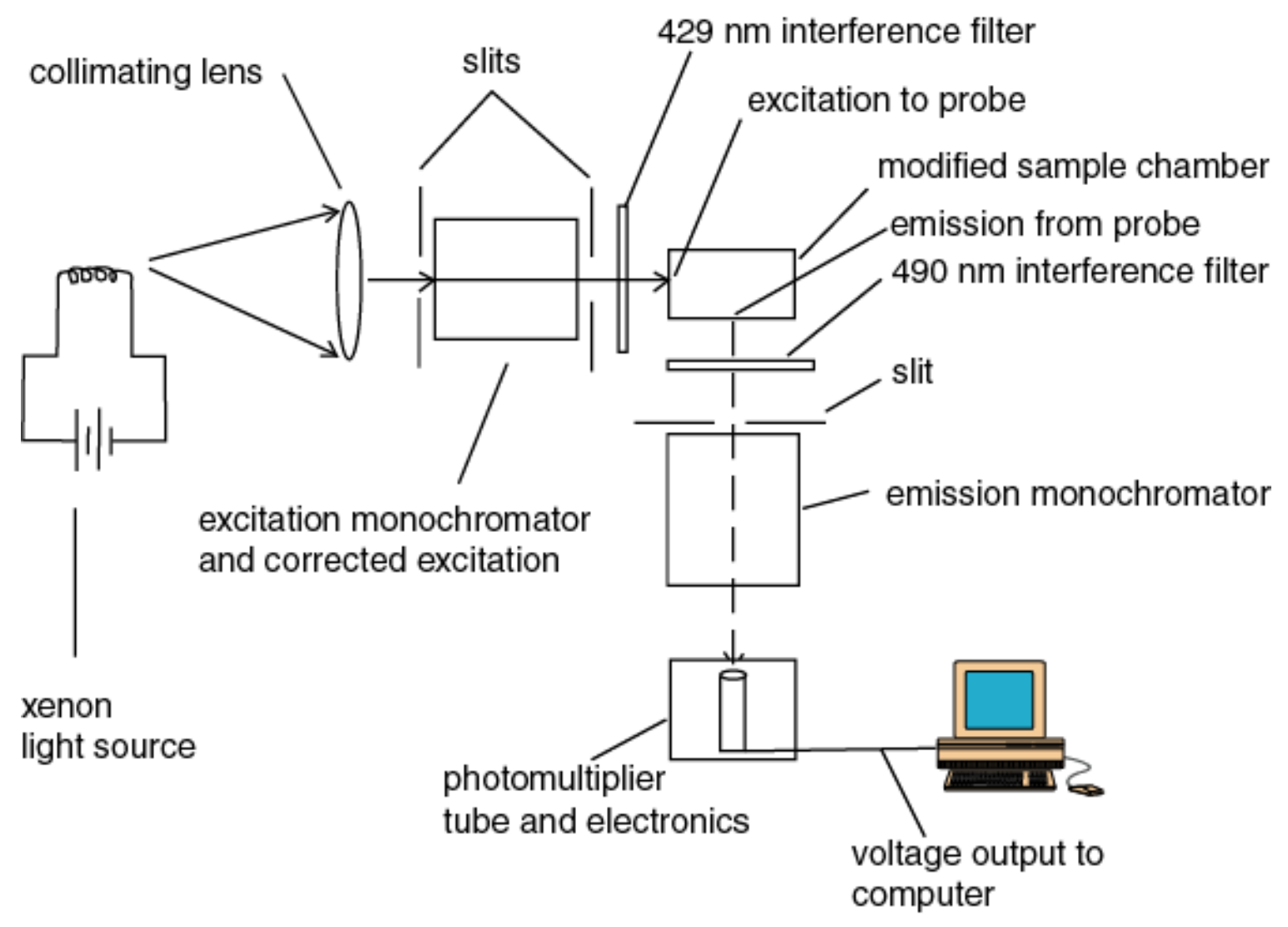

Fig. 3 Schematic of right angle spectrofluorometer 
contaminant deposition:

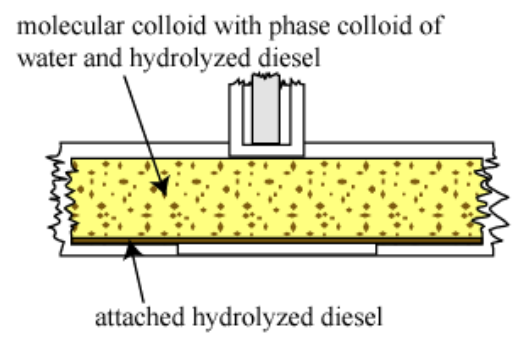

contaminant attachment:

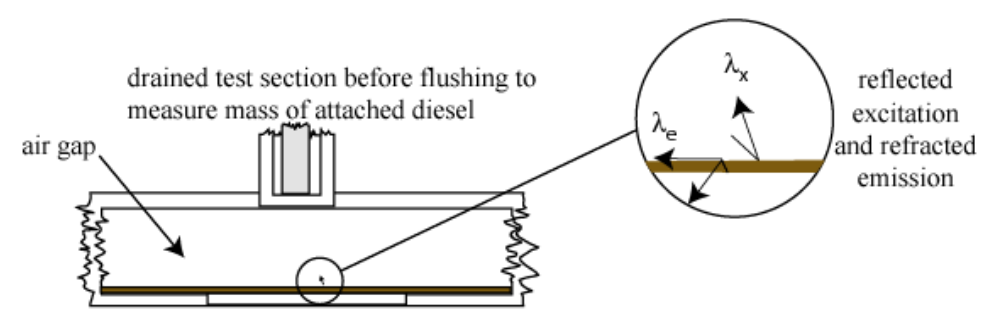

contaminant flush:

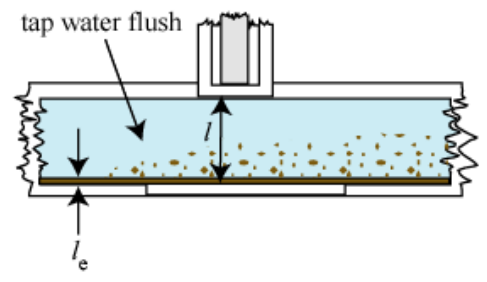

Fig. 4 Cross-sectional illustration of test section during contamination and flushing 


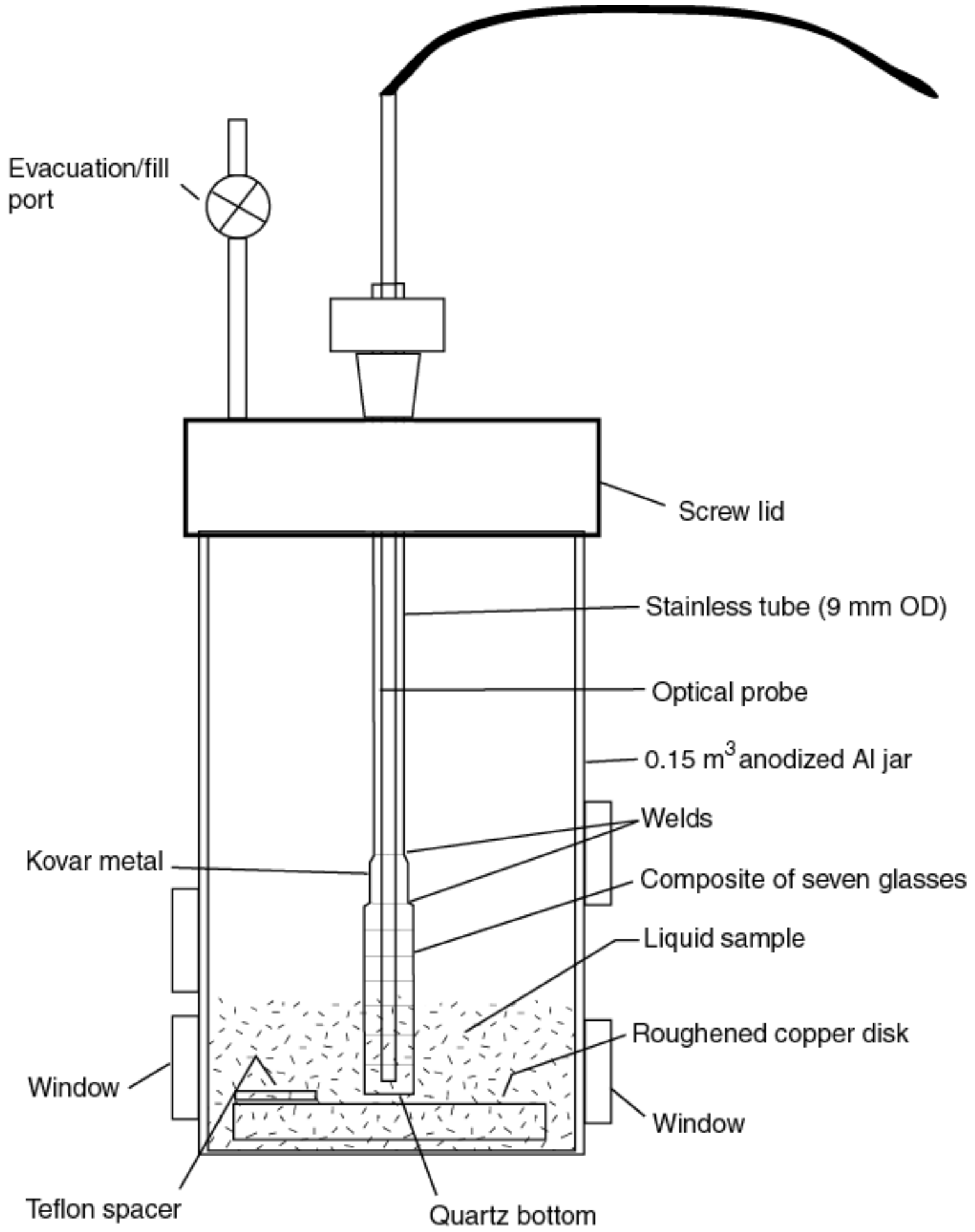

Fig. 5 Schematic of fluorescence/composition calibration jar 


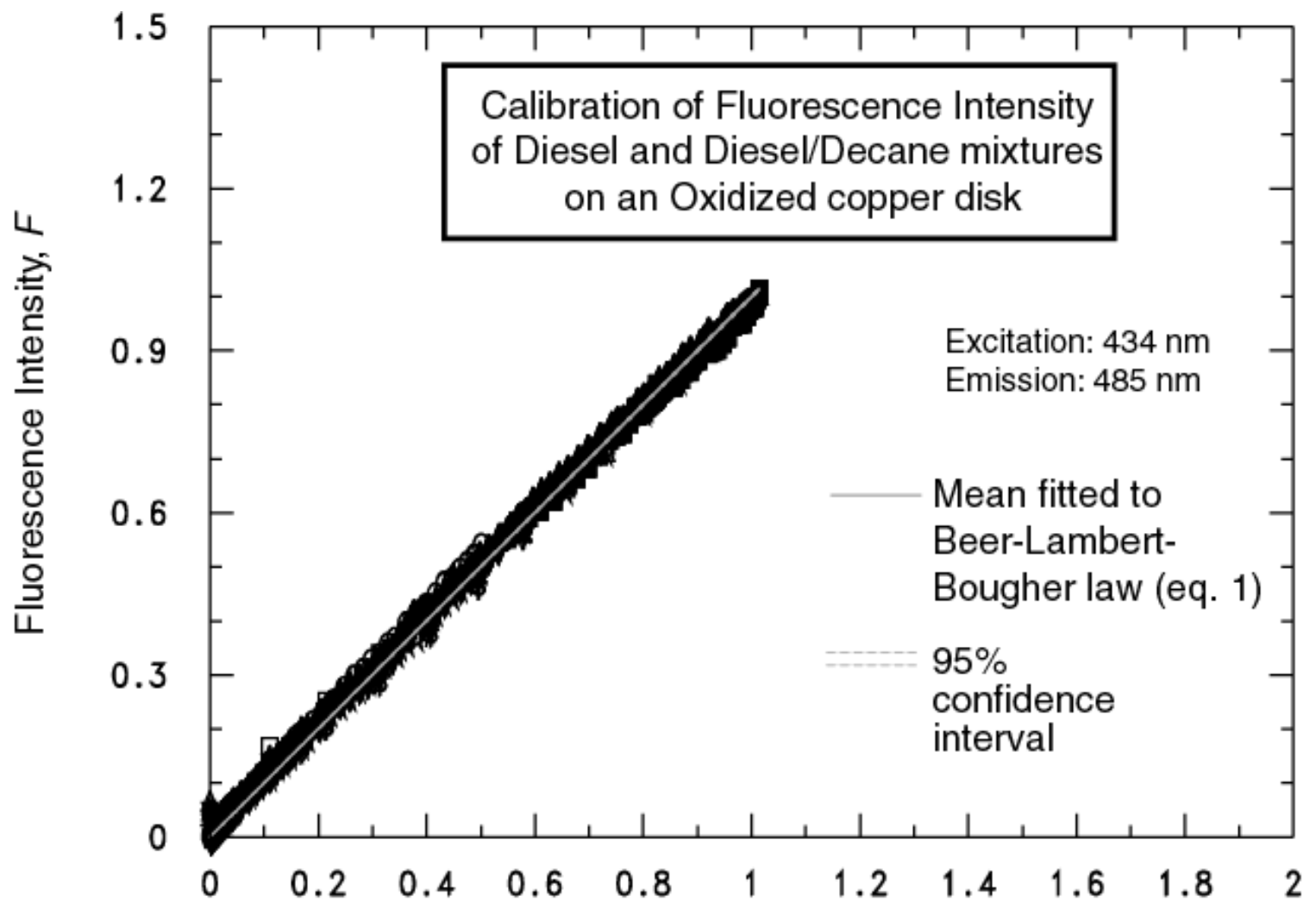

Predicted Fluorescence Intensity, $F$

Fig. 6 Overall calibration of Beer-Lambert Bougher law for diesel on copper disk 


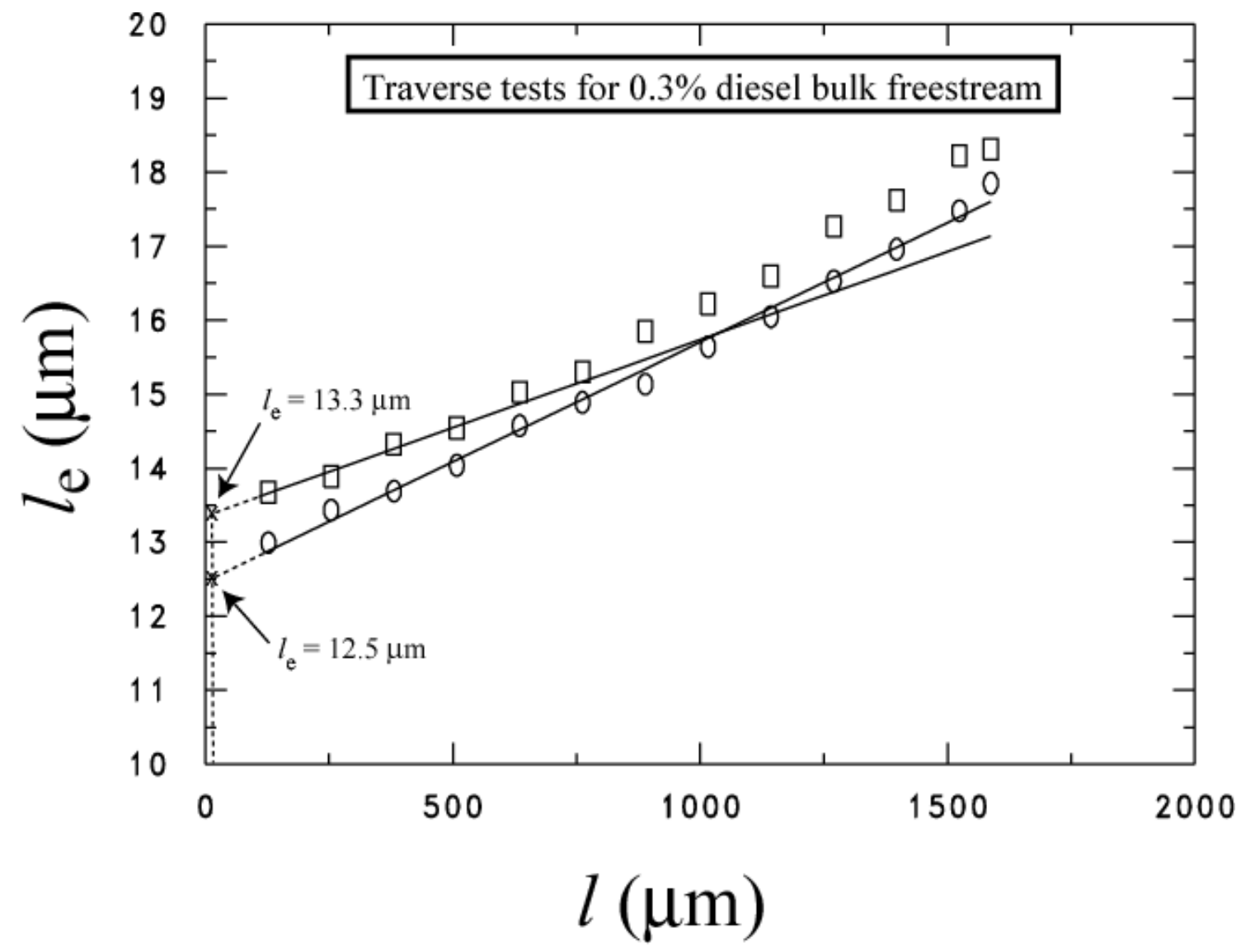

Fig. 7 Demonstration of excess layer thickness measurement 


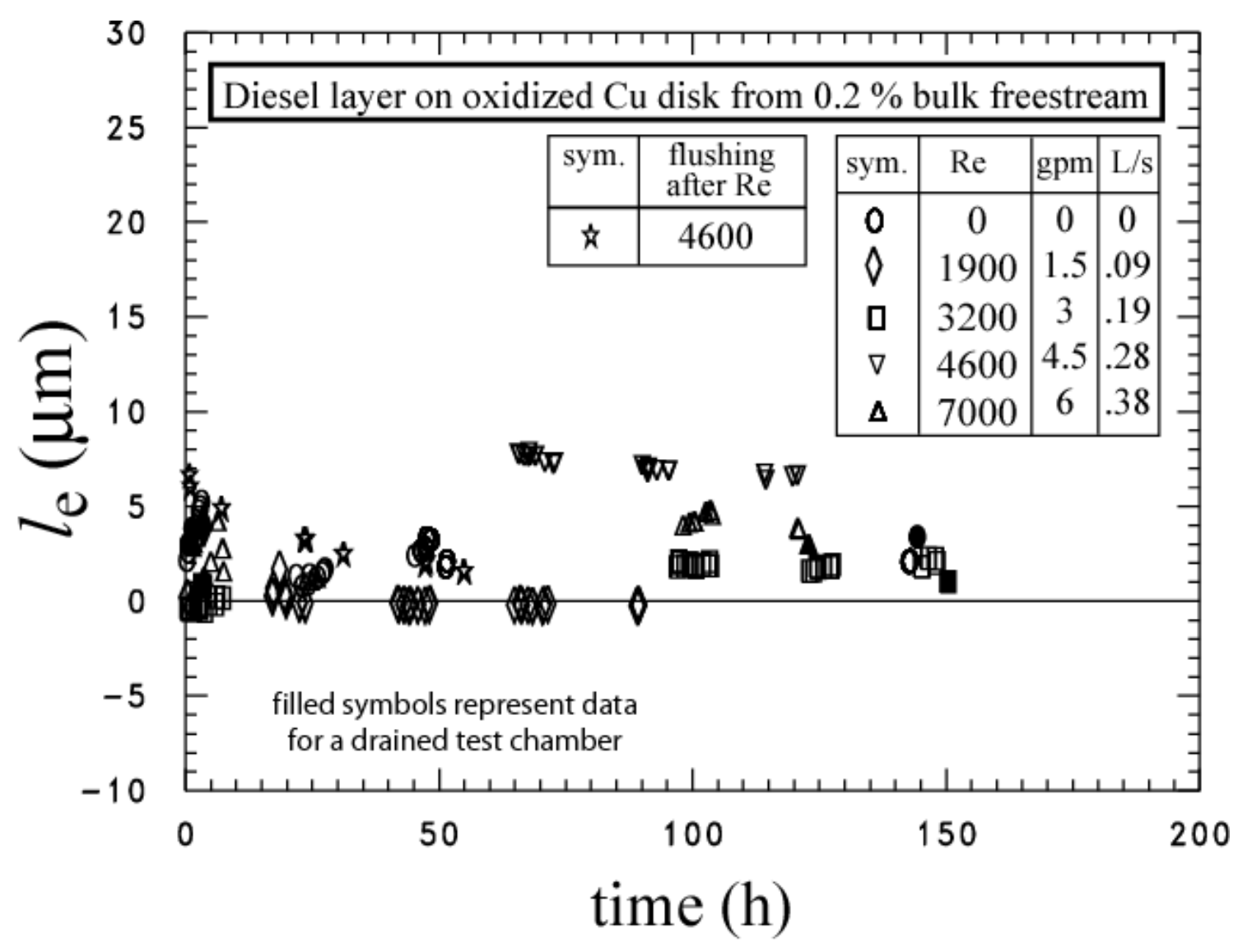

Fig. 8 Effect of exposure time and flow rate on thickness of the diesel excess layer for a $0.2 \%$ bulk freestream mass fraction 


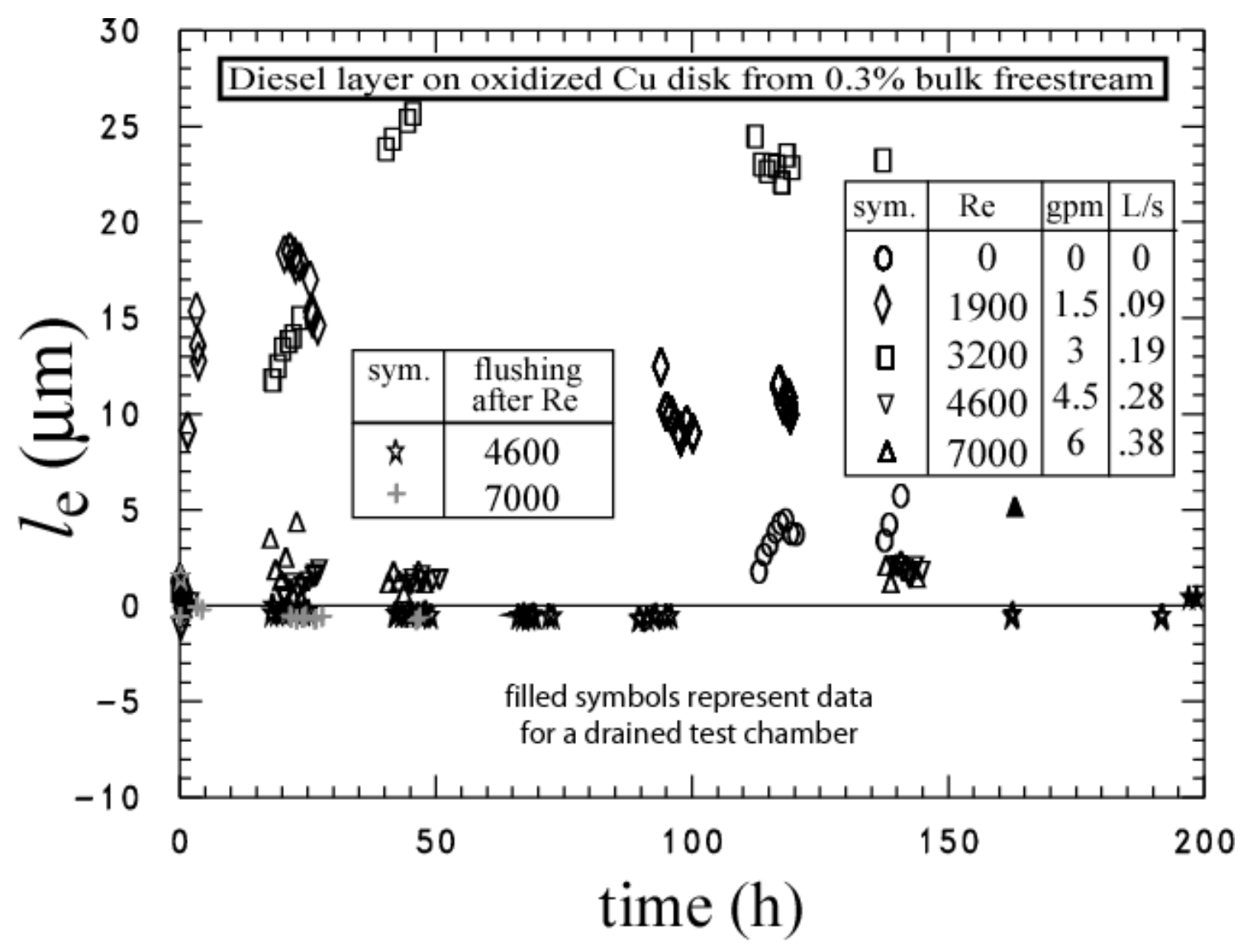

Fig. 9 Effect of exposure time and flow rate on thickness of the diesel excess layer for a $0.3 \%$ bulk freestream mass fraction 


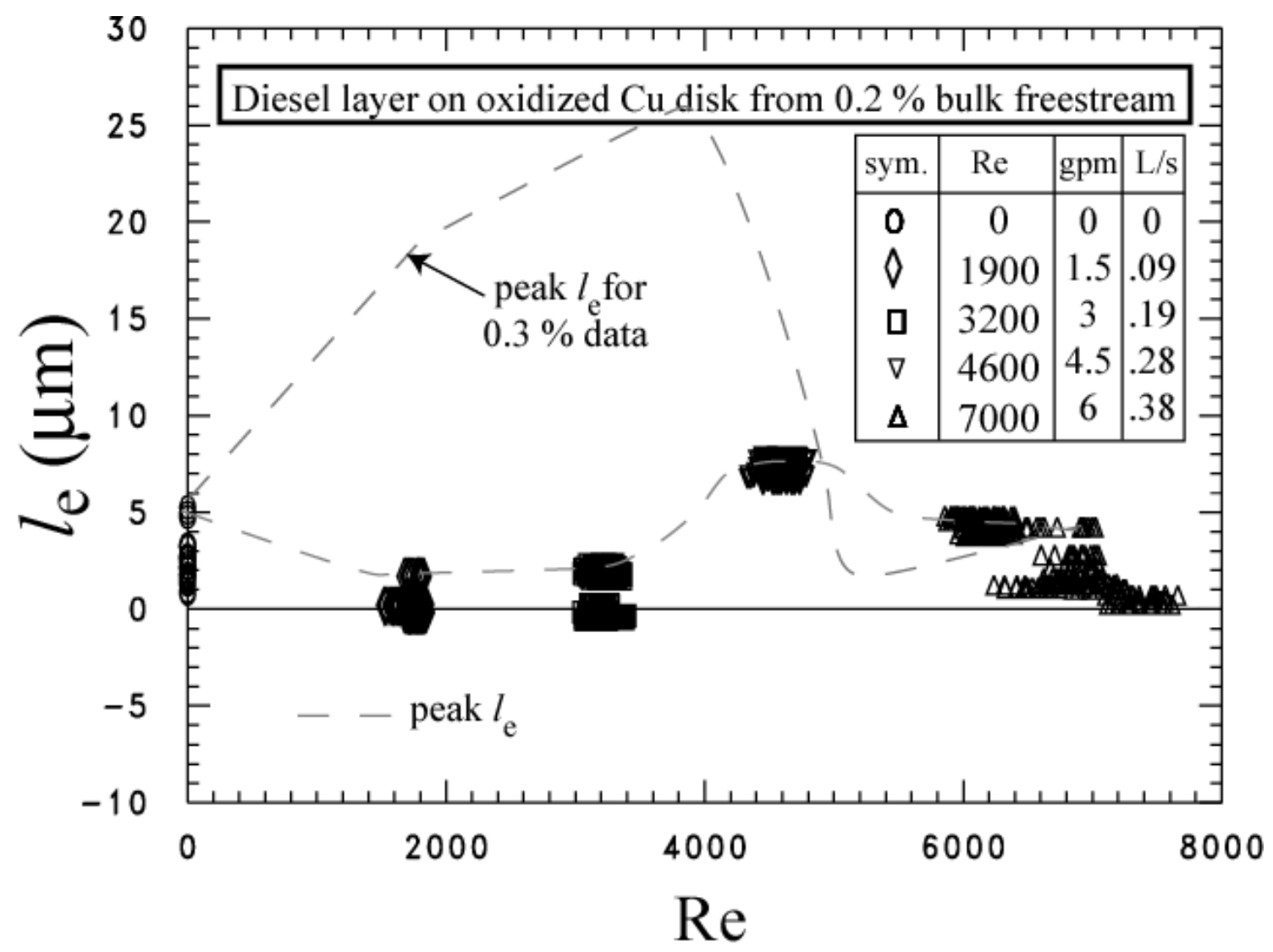

Fig. 10 Diesel excess layer thickness as a function of Re for water/diesel (99.8/0.2) 


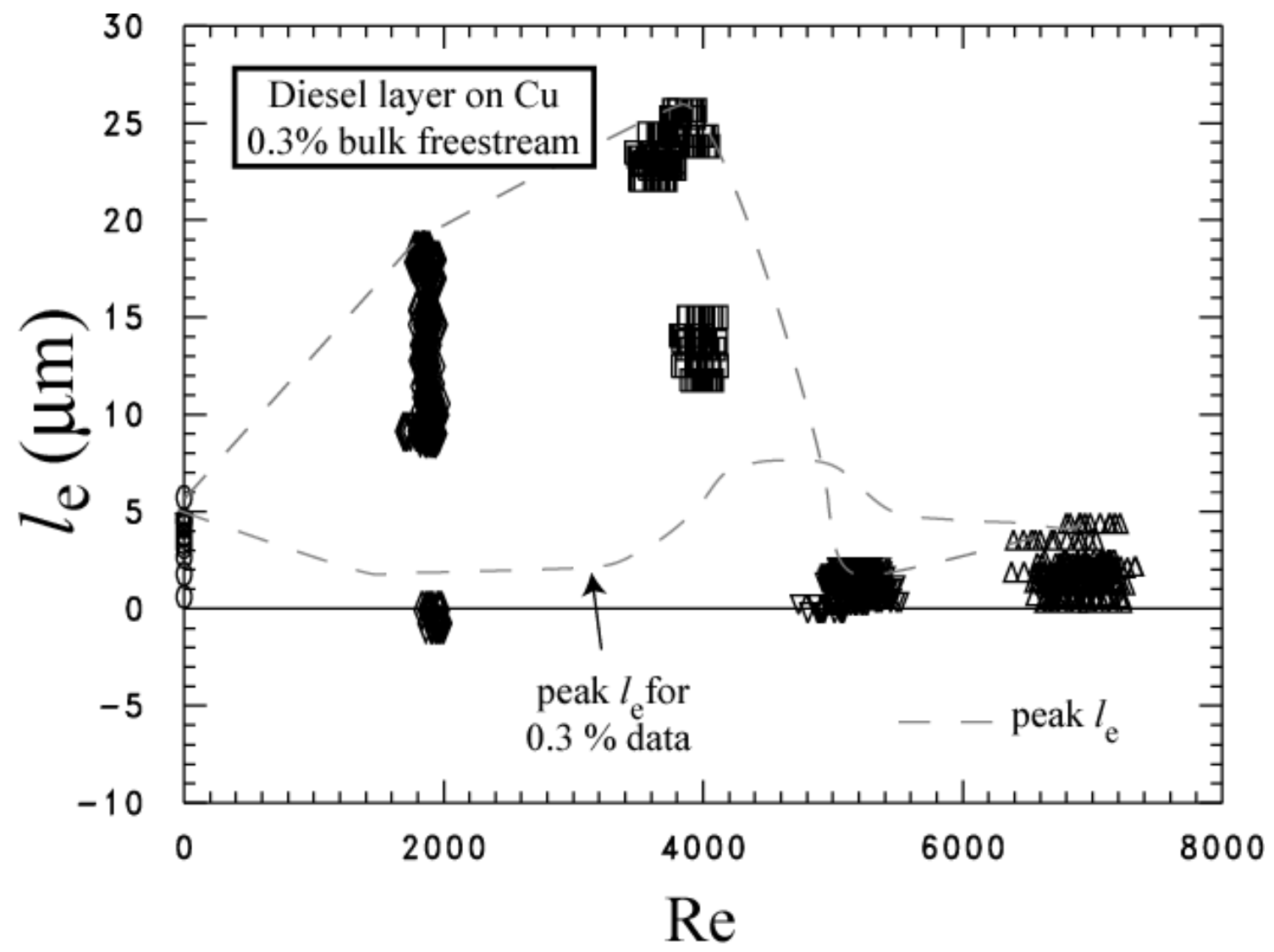

Fig. 11 Diesel excess layer thickness as a function of Re for water/diesel (99.7/0.3) 


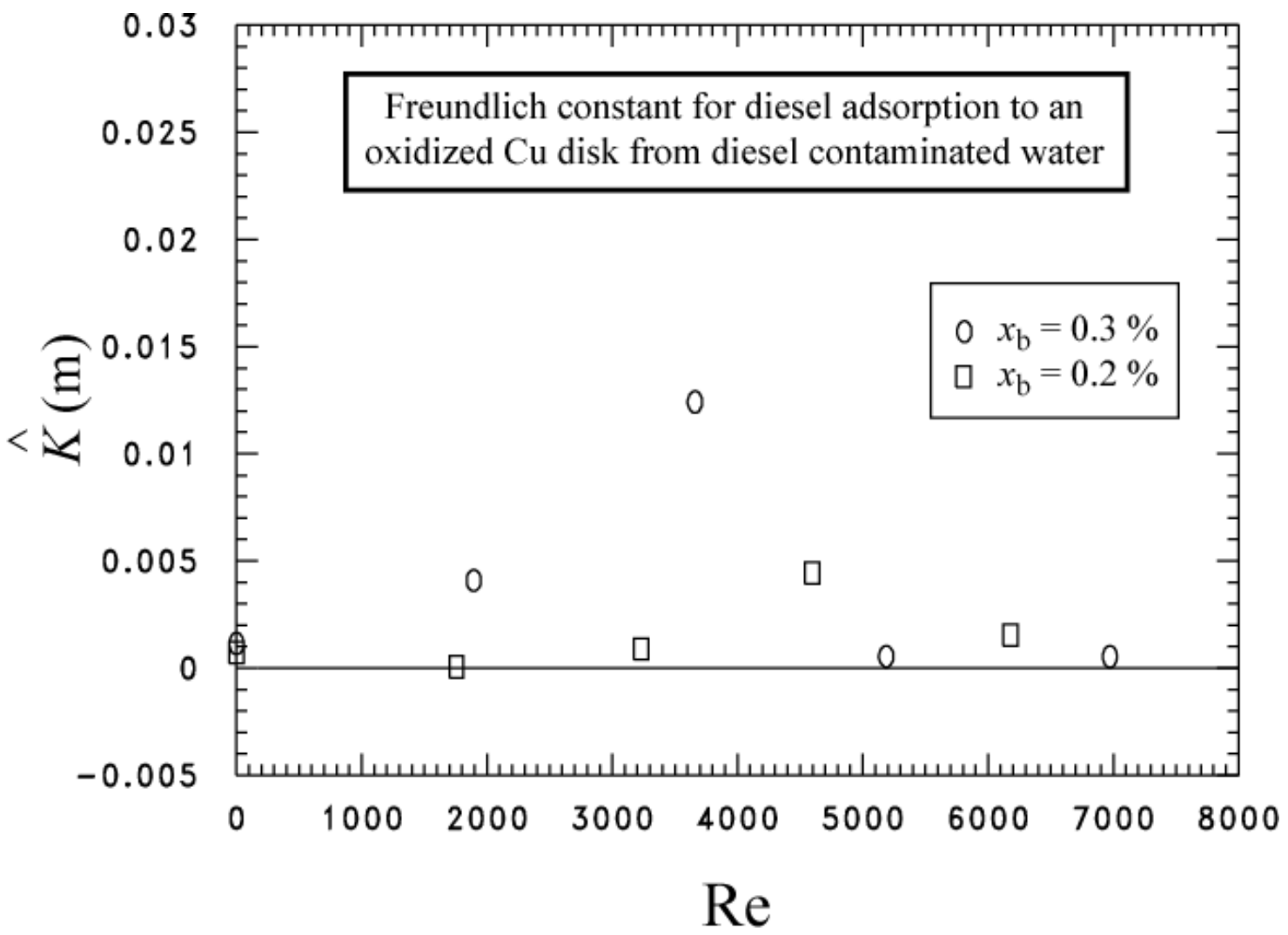

Fig. 12 Normalized Fruendlich constants for diesel adsorption to an oxidized $\mathrm{Cu}$ disk from diesel contaminated water 


\section{APPENDIX A: EXCITATION AND EMISSION WAVELENGTHS}

Appendix A describes the methodology used to select the excitation and emission wavelengths to ensure that a significant and measurable emission signal was obtained with no measurable overlap of the excitation and emission spectra.

The wavelengths for the excitation and emission light that gave the best compromise between emission intensity strength with minimal interference from the excitation were chosen based on the following analysis. Figure A.1 shows the analysis of the emission and excitation spectra of pure diesel in a cuvette. The test sample was placed directly in the sample chamber of the right angle spectrofluorometer. The excitation wavelength that produced the maximum fluorescence emission was iteratively found by scanning through both excitation and emission wavelengths. The excitation and emission wavelengths for diesel that produced the largest intensities were located approximately at $451 \mathrm{~nm}$ and $484 \mathrm{~nm}$, respectively.

It is immediately apparent that filtering of both the excitation and the emission is required to reduce the overlap of the two spectra. Because of the parallel light configuration of the probe incident to the test surface, both excitation and emission light will be introduced to the detector if the light is not filtered. A $429 \mathrm{~nm}$ bandpass filter was chosen to filter the excitation before it got to the test surface. And a $490 \mathrm{~nm}$ bandpass filter was used to filter the emission intensity before it was sent to the detector. Figure A.2 shows the filtered spectra from the optical probe in a jar of diesel. The filtering process as successfully separated the emission and excitation peaks ( $485 \mathrm{~nm}$ and $435 \mathrm{~nm}$, respectively) and removed the overlap of the two spectra. Because the interference filters have a finite bandwidth, an excitation filter centered at $450 \mathrm{~nm}$ would have resulted in some overlapping of the spectra. For this reason, a smaller wavelength was chosen for the excitation signal. 


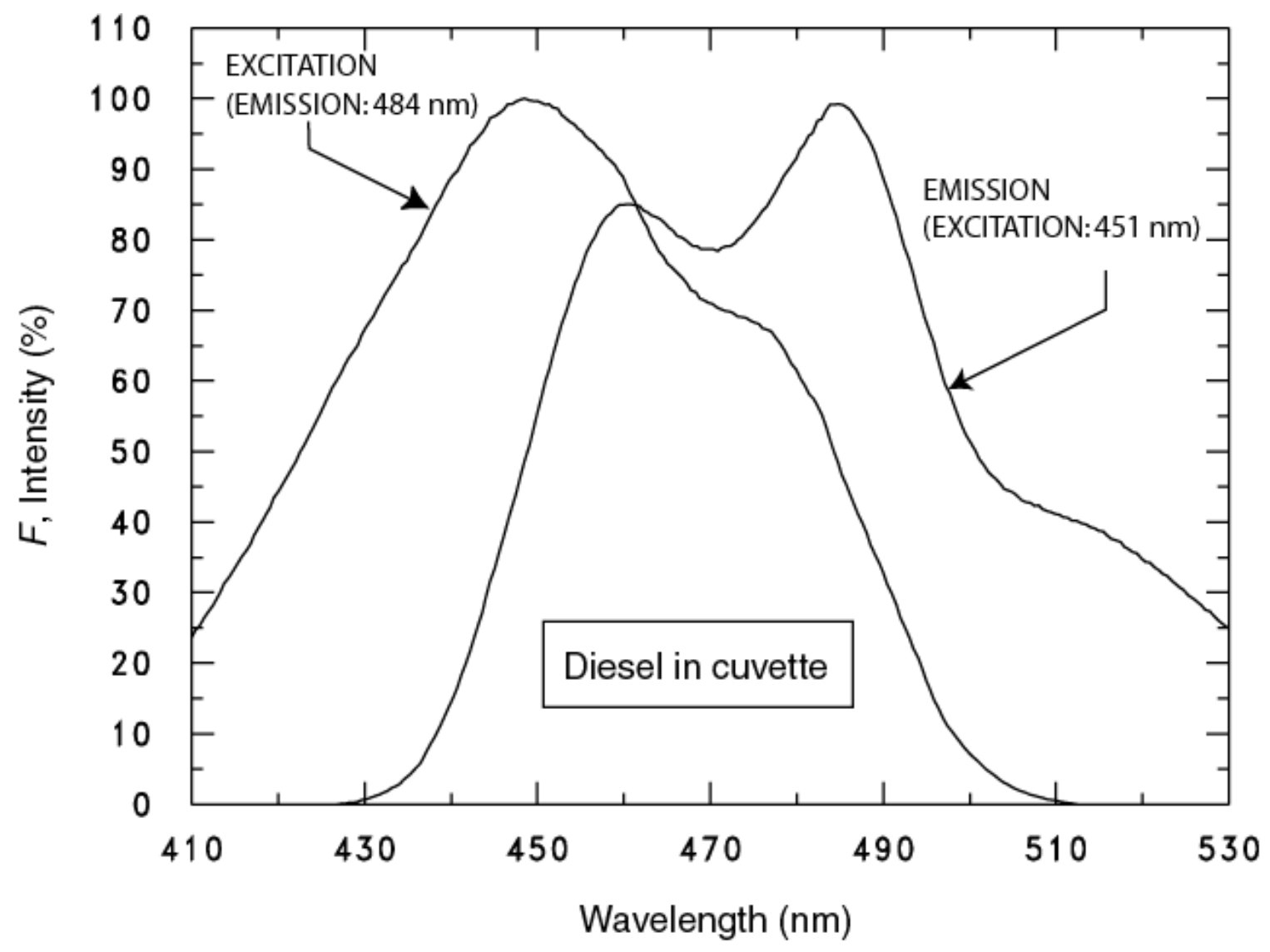

Fig. A.1 Emission and excitation spectra for diesel 


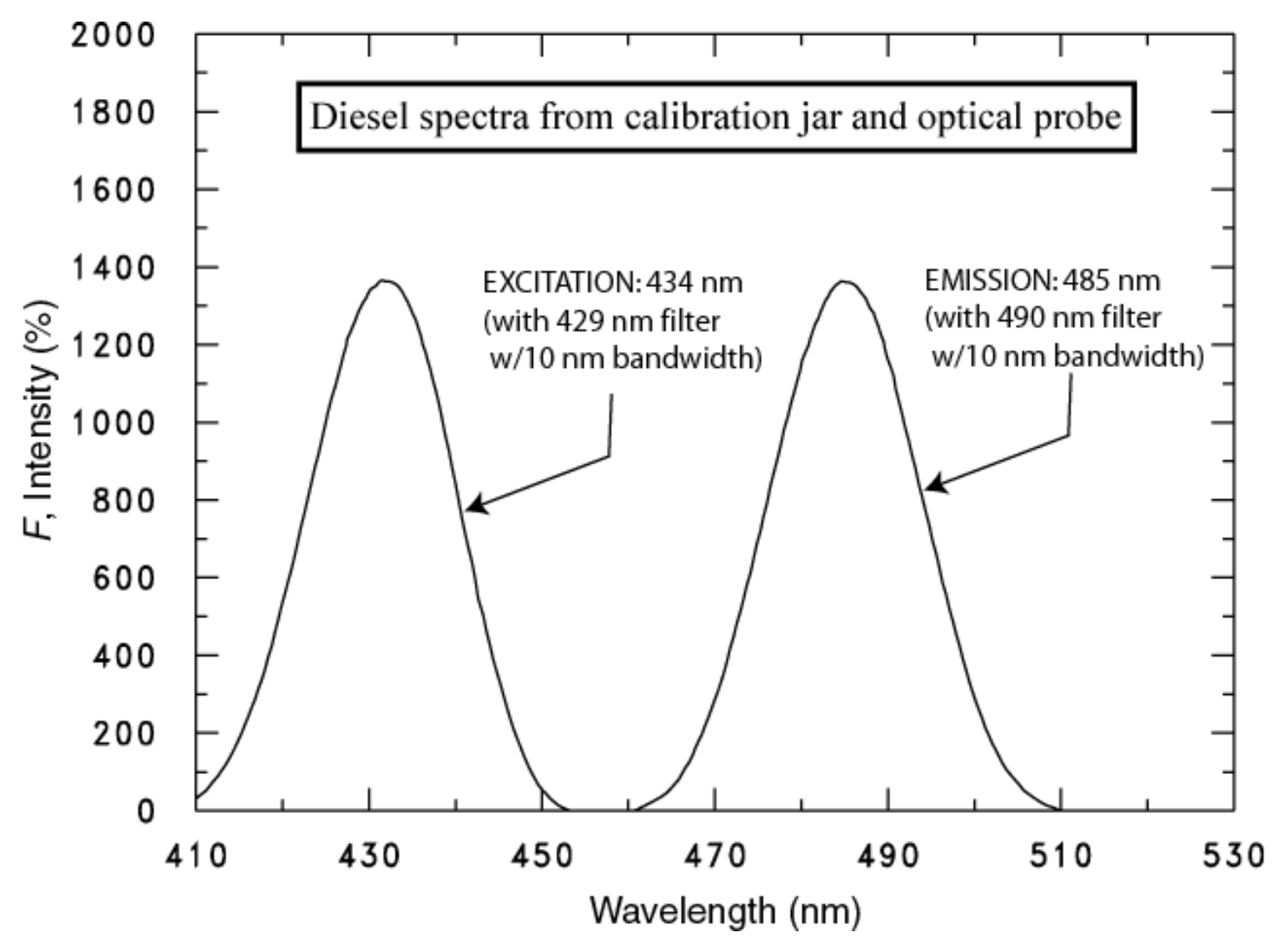

Fig. A.2 Filtered excitation and emission spectra for diesel 


\section{APPENDIX B: DIESEL PROPERTIES}

This appendix presents the measurements and the correlation of the density $\left(\rho_{\mathrm{d}}\right)$ and the kinematic viscosity $\left(v_{\mathrm{d}}\right)$ for the liquid \#2 diesel fuel used in this experiment.

\section{Liquid Density}

The density of the liquid diesel was measured as a function of temperature with a glass pycnometer. The pycnometer was factory instrumented with a glass mercury thermometer with a range of $14^{\circ} \mathrm{C}$ to $38^{\circ} \mathrm{C}$ in $0.2^{\circ}$ graduations, accurate to within $\pm 0.2 \mathrm{~K}$. The pycnometer was filled with distilled water and its volume was calculated from the known density of water. The volume was found over five trails to be $9.84 \mathrm{~mL}$ with a standard uncertainty of $0.01 \mathrm{~mL}$.

The pycnometer containing diesel was cooled in an ice bath and then removed from the bath and allowed to warm on the balance to room temperature over approximately one hour. The standard uncertainty of the balance was approximately $1 \mathrm{mg}$. The outside of the pycnometer was wiped clean before each measurement to remove the diesel that was expelled through the pipette due to volume expansion with temperature increase.

The Biot number for the warming pycnometer was estimated to be approximately 0.5 , which is greater than the recommended limit of 0.1 (Incropera and Dewitt, 1985) for a uniform temperature in fluid. It is difficult to estimate the error introduced in the measurements due to temperature gradients that existed in the diesel. However, the data regression shows that the residuals are independent of temperature, which suggests that the error due to temperature gradients in the liquid had a negligible effect on the density measurements.

Table B.1 shows the recorded measurements for two days. Equation B.1 gives the fit of the liquid diesel density $\left(\rho_{\mathrm{d}}\right)$ in $\mathrm{kg} / \mathrm{m}^{3}$ versus temperature $(T)$ in Kelvin:

$$
\rho_{\mathrm{d}}=1056.29-0.700 T
$$

The expanded uncertainty of the fit was approximately $\pm 0.2 \mathrm{~kg} / \mathrm{m}^{3}$ for $95 \%$ confidence.

\section{Kinematic Viscosity}

The kinematic viscosity of the liquid diesel $\left(v_{\mathrm{d}}\right)$ was measured at room temperature (approximately $297.6 \mathrm{~K}$ ) with a glass viscometer and found to be $3.93 \mu \mathrm{m}^{2} / \mathrm{s} \pm 0.024 \mu \mathrm{m}^{2} / \mathrm{s}$. Kinematic viscosity measurements from Simplex (2006) for \#2 diesel fuel shown in Table B.2 were used to obtain the trend of viscosity with respect to temperature. This was done by using the same slope of the linear $\ln v$ versus $T^{-1}$ fit for the Simplex (2006) data with an intercept that reproduced our single viscosity measurement. The following correlation reproduces the single room temperature value for our batch of diesel and temperature dependence of the Simplex (2006) diesel data:

$$
v_{\mathrm{d}}\left(\mathrm{m}^{2} / \mathrm{s}\right)=4.434 \times 10^{-9} e^{2020.17 / T(K)}
$$




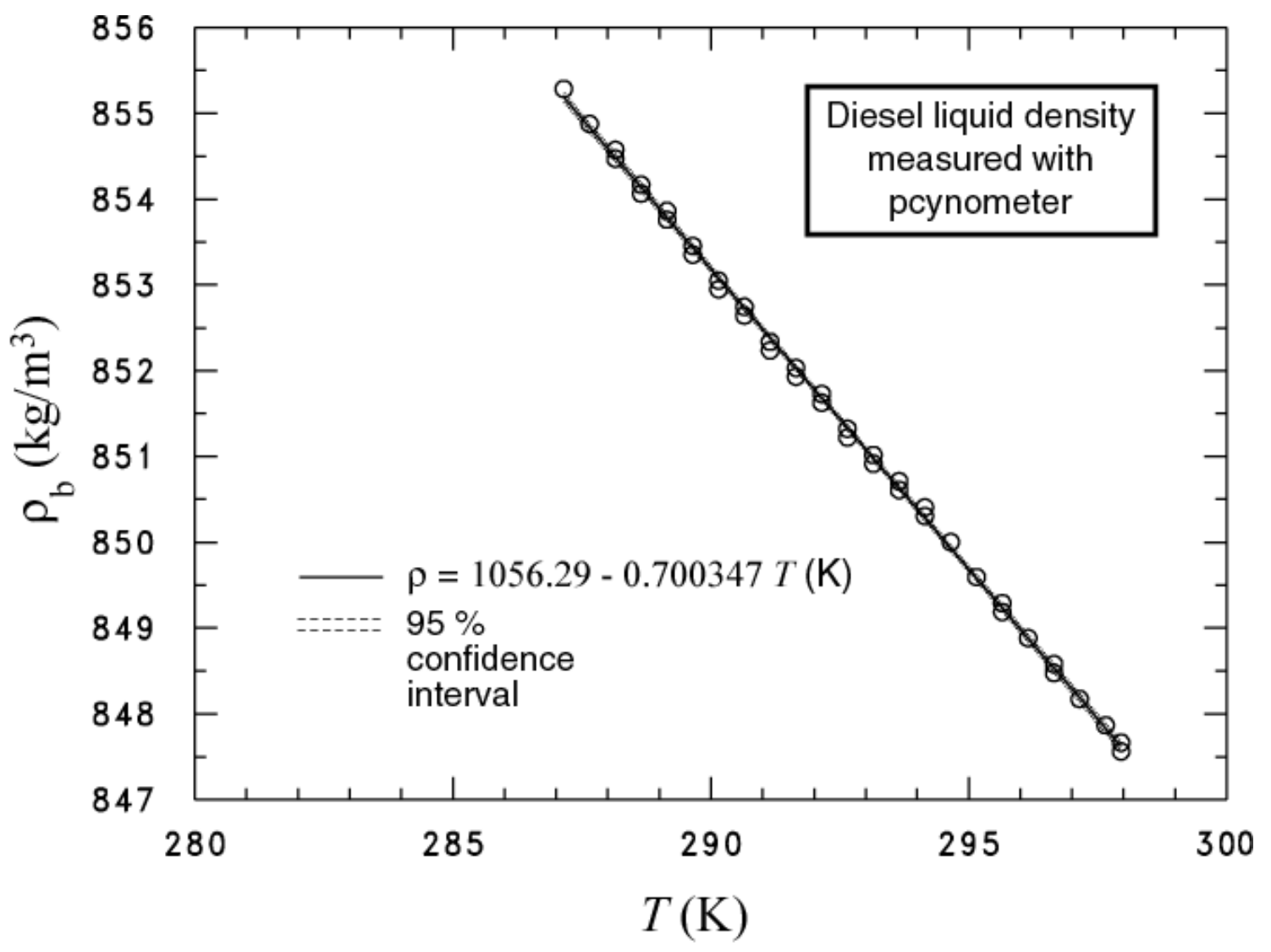

Fig. B.1 Measured liquid density of diesel and fit 
Table B.1 Diesel liquid density measurements (file:DieDen.dat)

\begin{tabular}{|c|c|c|c|}
\hline$T\left({ }^{\circ} \mathrm{C}\right)$ & $T(\mathrm{~K})$ & $\begin{array}{c}\text { diesel } \\
\text { mass } \\
\text { (g) }\end{array}$ & $\rho_{\mathrm{d}}\left(\mathrm{kg} / \mathrm{m}^{3}\right)$ \\
\hline 14.00 & 287.15 & 8.42 & 855.28 \\
\hline 14.50 & 287.65 & 8.41 & 854.88 \\
\hline 15.00 & 288.15 & 8.41 & 854.57 \\
\hline 15.50 & 288.65 & 8.40 & 854.17 \\
\hline 16.00 & 289.15 & 8.40 & 853.86 \\
\hline 16.50 & 289.65 & 8.40 & 853.46 \\
\hline 17.00 & 290.15 & 8.39 & 853.05 \\
\hline 17.50 & 290.65 & 8.39 & 852.74 \\
\hline 18.00 & 291.15 & 8.39 & 852.34 \\
\hline 18.50 & 291.65 & 8.38 & 852.03 \\
\hline 19.00 & 292.15 & 8.38 & 851.73 \\
\hline 19.50 & 292.65 & 8.38 & 851.32 \\
\hline 20.00 & 293.15 & 8.37 & 851.02 \\
\hline 20.50 & 293.65 & 8.37 & 850.71 \\
\hline 21.00 & 294.15 & 8.37 & 850.41 \\
\hline 21.50 & 294.65 & 8.36 & 850.00 \\
\hline 22.00 & 295.15 & 8.36 & 849.59 \\
\hline 22.50 & 295.65 & 8.36 & 849.29 \\
\hline 23.00 & 296.15 & 8.35 & 848.88 \\
\hline 23.50 & 296.65 & 8.35 & 848.48 \\
\hline 24.00 & 297.15 & 8.35 & 848.17 \\
\hline 24.50 & 297.65 & 8.34 & 847.87 \\
\hline 24.80 & 297.95 & 8.34 & 847.66 \\
\hline 14.00 & 287.15 & 8.42 & 855.28 \\
\hline 14.50 & 287.65 & 8.41 & 854.88 \\
\hline 15.00 & 288.15 & 8.41 & 854.47 \\
\hline 15.50 & 288.65 & 8.40 & 854.06 \\
\hline 16.00 & 289.15 & 8.40 & 853.76 \\
\hline 16.50 & 289.65 & 8.40 & 853.35 \\
\hline 17.00 & 290.15 & 8.39 & 852.95 \\
\hline 17.50 & 290.65 & 8.39 & 852.64 \\
\hline 18.00 & 291.15 & 8.39 & 852.24 \\
\hline 18.50 & 291.65 & 8.38 & 851.93 \\
\hline 19.00 & 292.15 & 8.38 & 851.63 \\
\hline 19.50 & 292.65 & 8.38 & 851.22 \\
\hline 20.00 & 293.15 & 8.37 & 850.91 \\
\hline 20.50 & 293.65 & 8.37 & 850.61 \\
\hline 21.00 & 294.15 & 8.37 & 850.30 \\
\hline 21.50 & 294.65 & 8.36 & 850.00 \\
\hline 22.00 & 295.15 & 8.36 & 849.59 \\
\hline 22.50 & 295.65 & 8.36 & 849.19 \\
\hline 23.00 & 296.15 & 8.35 & 848.88 \\
\hline 23.50 & 296.65 & 8.35 & 848.58 \\
\hline 24.00 & 297.15 & 8.35 & 848.17 \\
\hline 24.50 & 297.65 & 8.34 & 847.87 \\
\hline 24.80 & 297.95 & 8.34 & 847.56 \\
\hline
\end{tabular}

Table B.2 Diesel \#2 liquid kinematic viscosity measurements (Simplex, 2006)

\begin{tabular}{|c|c|c|c|}
\hline$T\left({ }^{\circ} \mathrm{F}\right)$ & $T(\mathrm{~K})$ & $v_{\mathrm{d}}(\mathrm{SUS})$ & $v_{\mathrm{d}}\left(\mu \mathrm{m}^{2} / \mathrm{s}\right)$ \\
\hline 30 & 272.04 & 138 & 27.6 \\
\hline 60 & 288.71 & 70 & 14 \\
\hline 80 & 299.82 & 53.6 & 10.72 \\
\hline 100 & 310.93 & 45.5 & 9.1 \\
\hline 130 & 327.59 & 39 & 7.8 \\
\hline
\end{tabular}




\section{APPENDIX C: HYDROLYZED DIESEL}

Figure C.1 compares the fluorescent emission spectrum for pure diesel to that of the hydrolyzed diesel in water mixture as taken from the reservoir of the test chamber. Both fluids where excited in quartz cuvettes at a wavelength of $451 \mathrm{~nm}$ with $2.5 \mathrm{~nm}$ slits in the spectrofluorometer. The fluid from the test reservoir was mostly the bulk phase of the water where the hydrolyzed diesel was stably suspension within the bulk water and at approximately $0.15 \%$ mass fraction of diesel. The mass fraction was determined from the relative peak intensities of the reservoir fluid to that of the pure diesel for these cuvette tests. No interference filters were used in the sample chamber of the spectrofluorometer. Evidence for a chemical breakdown of the diesel is based on the fact that the peak fluorescence emission for the emulsified water diesel exists at a wavelength that is $25 \mathrm{~nm}$ greater than that of pure diesel.

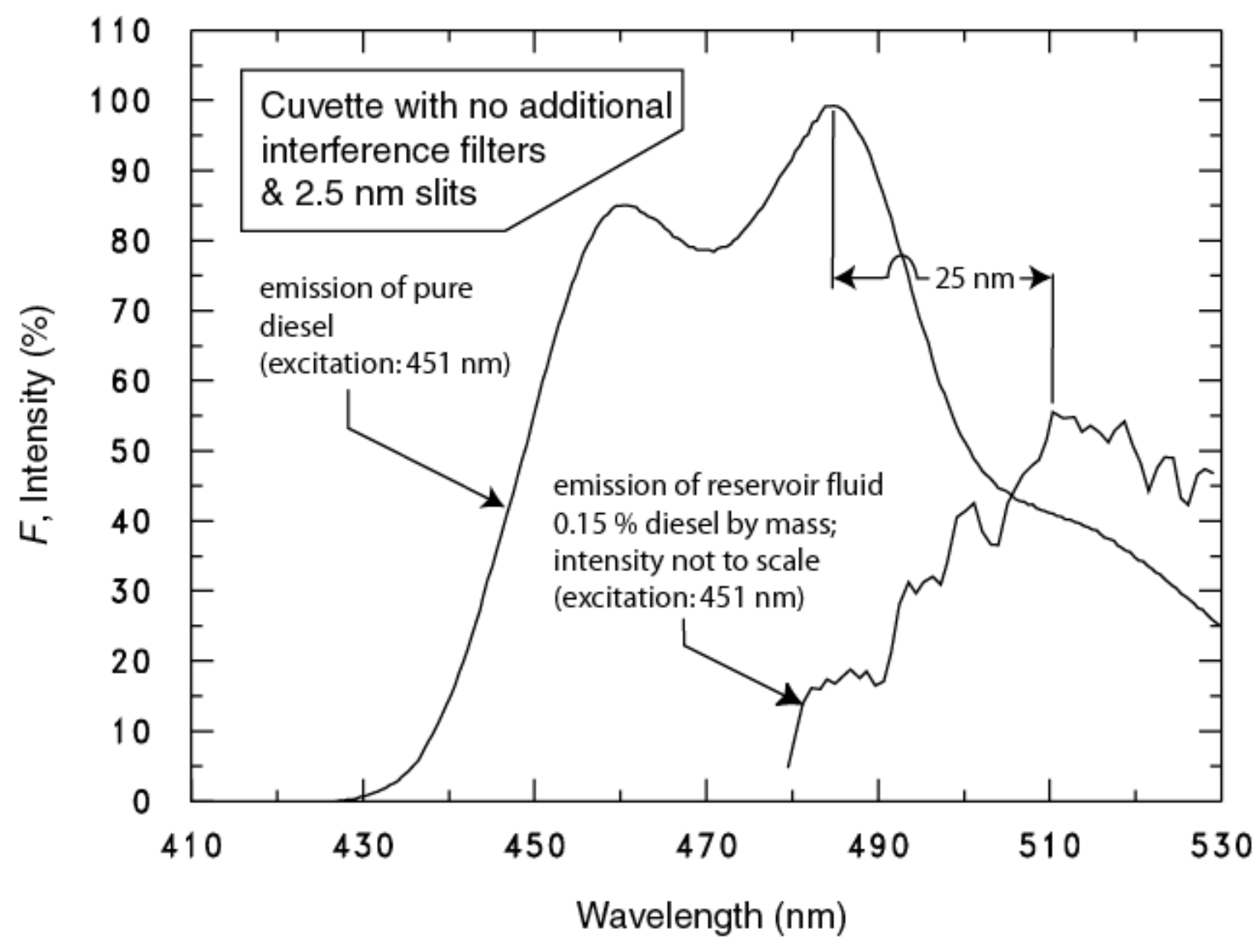

Fig. C.1 Fluorescent emission spectra for pure diesel and hydrolyzed reservoir test fluid 


\section{APPENDIX D: FLUORESCENT TEMPERATURE DEPENDENCE}

This appendix presents the measurements and the methodology that were used to determine the coefficient of temperature dependence ( $\beta$ ) for the fluorescent intensity of diesel (Kedzierski, 2003). All of the measurements and settings were made with the excitation set to $434 \mathrm{~nm}$ and the emission measured at $485 \mathrm{~nm}$ with the additional spectrofluorometer filters in place as described in Appendix A. Pure diesel was cooled from nominally $303 \mathrm{~K}$ to $279 \mathrm{~K}$ for two sets of 500 measurements in a temperature controlled liquid bath. The temperature of the mixture was measured with a sheathed thermocouple that was in the controlled temperature bath with the maximum- and zero-jars. The fluorescence intensity of the maximum-jar as a function of bath temperature is given in Fig. D.1.

The temperature dependence of the fluorescence can be expressed as the ratio of the fluorescence intensity at the bath temperature $\left(F_{T_{\mathrm{b}}}\right)$ to the intensity evaluated at the test section temperature $\left(F_{T_{\mathrm{T}}}\right)$ for all other variables held constant (Kedzierski, 2003):

$$
\frac{F_{T_{\mathrm{b}}}}{F_{T_{\mathrm{T}}}}=e^{\beta\left(T_{\mathrm{b}}-T_{\mathrm{T}}\right)}
$$

where the coefficient of temperature dependence of the fluorescence $(\beta)$ was found to be approximately 0.00156 for the diesel data set shown in Fig. D.1.

Equation D.1 is used to account for any difference between the temperature of the bath that holds the fluorescent standard jars and the temperature of the test section. The target test section fluid temperature for the contamination tests is the same as the fluorescent standard bath temperature. As a result, for approximately $85 \%$ of the contamination measurements, the test section temperature differed no more than $\pm 1 \mathrm{~K}$ from the bath temperature, which would have resulted in an adjustment in the fluorescence with eq. (D.1) by no more than $\pm 0.2 \%$. The largest difference between the temperature of the jar bath and the average temperature of the test section for the contamination tests was approximately $4.5 \mathrm{~K}$, which corresponds to a $0.3 \%$ correction of the fluorescence intensity. Flushing tests required larger corrections via eq. (D.1). Flushing was done with house tap water that was as much as $10 \mathrm{~K}$ colder than the jar bath temperature, which resulted in a maximum correction of $1.5 \%$ of the fluorescence intensity. 


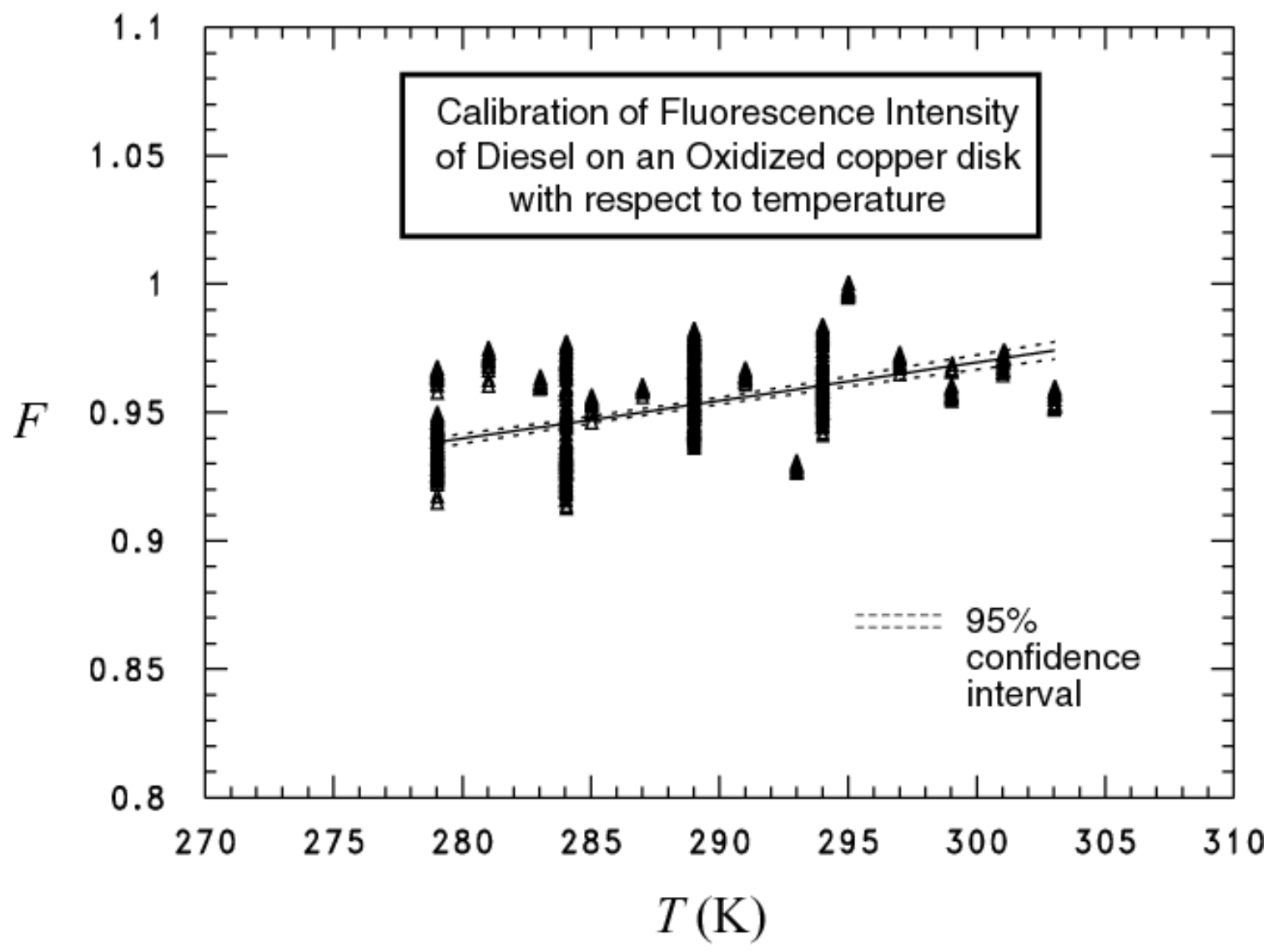

Fig. D.1 Temperature dependence of diesel fluorescence 


\section{APPENDIX E: FLUORESCENCE CALIBRATION}

This appendix provides more detail on the procedure that was developed and used to calibrate the fluorescence intensity of diesel against mass fraction, path length, air gap, and fluid properties. All calibration measurements were done on a copper disk that was flattened from a pipe and evenly oxidized by electrolysis. Appendix I shows how the functionality of the spectrofluorometer was verified.

Figure E.1 shows the calibration of the fluorescence intensity of diesel against diesel mass fraction for fixed path length. The temperature of the diesel in the $150 \mathrm{~mL}$ calibration jar (shown in Fig. 6) was held constant at approximately $294 \mathrm{~K}$. The diesel was mixed with nonfluorescent n-decane in order to dilute the diesel to the desired mass fraction. Unlike water, n-decane was miscible with diesel. The distance between the top of the calibration disk and the bottom of the quartz tube (the path length) was set and fixed with the aid of a $1.6 \mathrm{~mm}$ gauge block. For these conditions, the fluorescence intensity was fitted linearly with respect to the diesel mass fraction to within a residual standard deviation of $\pm 1.2 \%$.

Figure E.2 shows the calibration of the fluorescence intensity of diesel against the path length through the diesel for neat diesel (for fixed $x_{\mathrm{b}}=1$ ). As in the previous mass fraction calibration described above, the temperature of the diesel was held constant at approximately $294 \mathrm{~K}$. This second calibration method was used to determine the effect of the proximity of the incident light $\left(I_{o}\right)$ via changing its path length $(l)$. As shown in Fig. 2, a linear positioning device with a graduated knob was used to locate the quartz tube relative to the test surface and thus measure and set the path length of the incident light through the diesel. The measured fluorescent intensity versus the path length was non-linear as shown in Fig. E.2. The calibrations given in Figs. E.1 and E.2 were combined with an exponential representation of $I_{0}$ as a function of $l$ to give the total calibration as given in eq. (4).

Figure E.3 shows the calibration measurements that were used to determine the effect of an air gap above the test surface. This method served as a secondary measurement check because it was desired to have a technique that did not require the test section to be filled with test fluid. Because of the mismatch in the index of refraction between the quartz, the air, and the diesel film, the intensity incident to the diesel for when an air-gap existed differed from that for when fluid filled the space between the test surface and the bottom of the quartz probe. To determine the effect of incident intensity reflections from interfaces exposed by the air gap, the probe was traversed above a diesel film of fixed thickness. Because the amount of fluorescent material remained fixed for these tests, the magnitude of the measured intensity was attributed to change in the magnitude of the incident intensity due to it proximity to the diesel film.

Figure E.3. shows measurements with and with out air gaps below the quartz probe. As expected, measurements with no air gap are shown to lie on the eq. (4) calibration.

Measurements taken with an air gap reside to the right of the eq. (4) calibration in a stratum of nearly linear data grouped by different diesel film thickness. For these measurements, the intensity is shown to increase slightly has the probe approaches the diesel film. From the airgap data, it was observed that $\frac{1}{F} \frac{d F}{d l}$ was approximately constant for all ranges of the $F$ and $l$ 
traverse data for each group of fixed diesel film thickness. The value of $F$ used in this product for each group was extrapolated to the calibration line for no-air-gap $\left(F_{\mathrm{ng}}\right)$. The gradient was calculated using the air-gap measurements, i.e., $\frac{d F_{a g}}{d l}$. The average ratio between $F_{\text {ng }}$ and the air-gap gradient for all the groups was:

$$
\frac{1}{F_{n g}} \frac{d F_{a g}}{d l}=-82.57 \mathrm{~m}^{-1}
$$

From the integration of eq. (E.1) it can be seen that the dependency of the intensity is exponential with path length. Considering that this dependency represents how $I_{0}$ changes with proximity to the test surface, an exponential term with respect to path length was used to represent $I_{0}$ in the full calibration eq. (4).

The diesel film thickness using the air-gap fluorescent gradient can be solved for by substituting eq. (4) into eq. (E.1) for the $F_{\text {ng: }}$ :

$$
l_{e}=\frac{-0.0121 \mathrm{~m} \frac{d F_{\mathrm{ag}}}{d l}}{2.3 I_{o} \Phi \varepsilon M_{\mathrm{c}}^{-1}}=\frac{-0.0115 \mathrm{~m}^{-1} \mathrm{~kg} \frac{d F_{\mathrm{ag}}}{d l} e^{209.23 \mathrm{~m}^{-1} l}}{x_{\mathrm{b}} \rho_{\mathrm{b}}}
$$




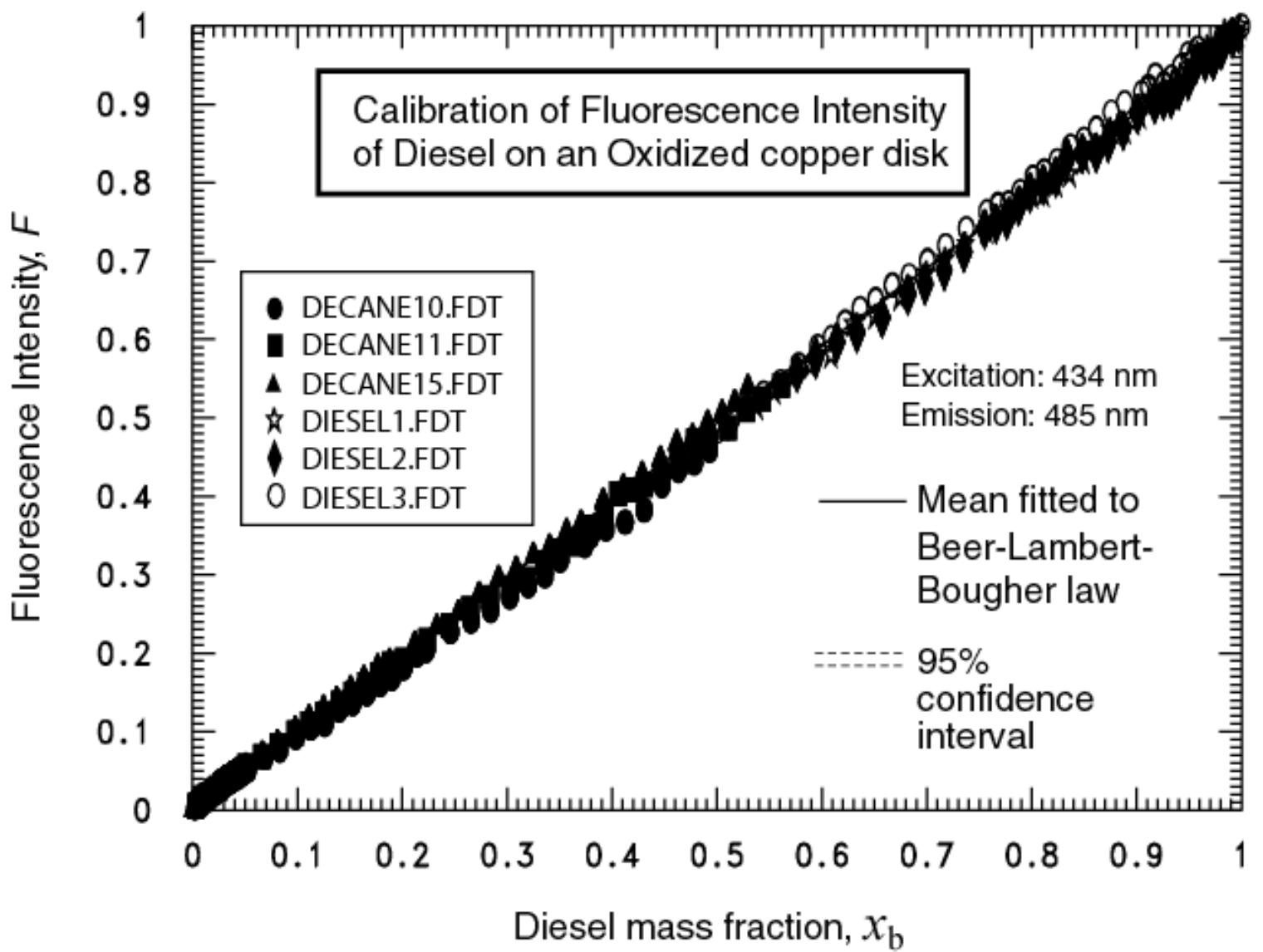

Fig. E.1 Calibration of diesel fluorescence against diesel mass fraction for different runs 


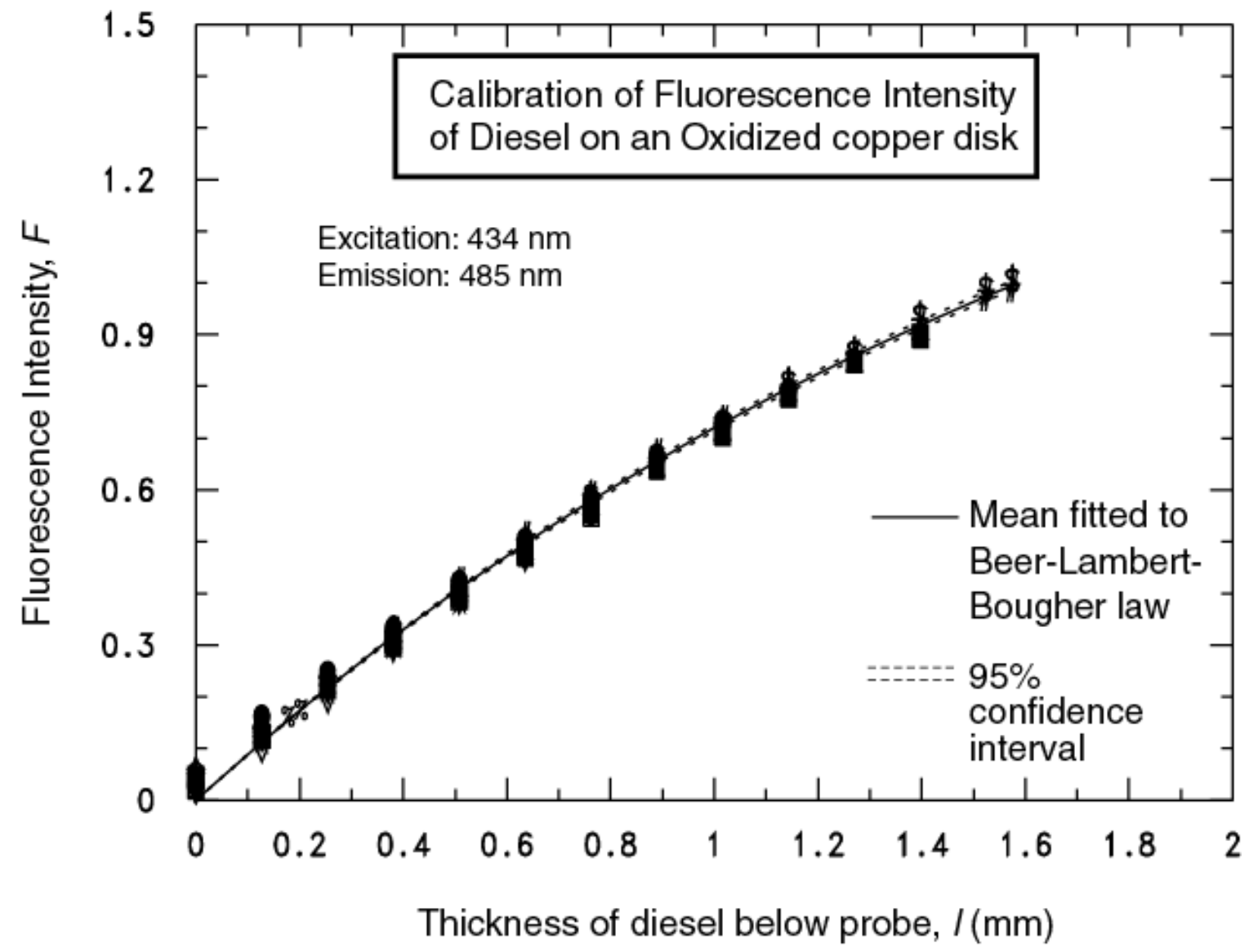

Fig. E.2 Calibration of fluorescence of diesel against path length for different runs 


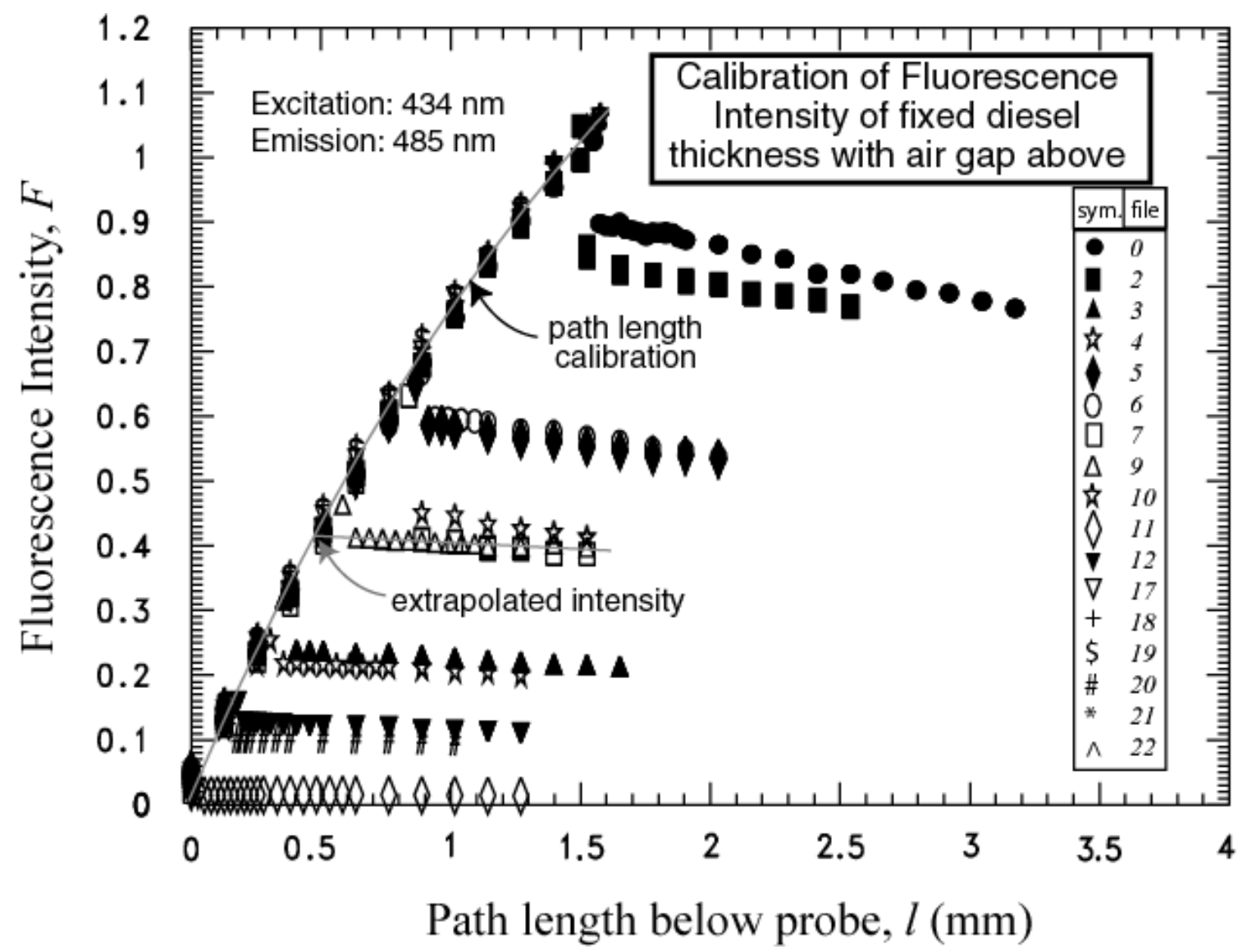

Fig. E.3 Calibration of diesel fluorescence intensity for fixed film thickness and air gap between quartz tube and liquid film 


\section{APPENDIX F: LINEAR BEER LAW}

This appendix justifies the use of the linear form of the Beer-Lambert-Bougher law (Amadeo et al., 1971) for the fluorescence calibration of the diesel mass. Regression of the calibration varied mass fraction measurements to the exponential and complete form of the BeerLambert-Bougher law:

$$
F=I_{o} \Phi\left(1-10^{-\varepsilon c l}\right)
$$

resulted in a residual standard deviation between the measurements and the fit of 0.0156 . Considering that the residual standard deviation of the linear fit (eq. 3) was marginally less (0.0151) than that of eq. (F.1), the linear model represents the calibration data just as well as the complete model.

Further justification for the use of eq. (F.1) can be obtained from the general knowledge of fluorescence characteristics. According to Herman (1998), fluorescence remains directly proportional to absorbance $(\varepsilon c l)$ as long as it is small, i.e., $\varepsilon c l<0.05$. Figure F.1 plots the absorbance against the mass fraction for both the mass fraction and the path length calibration measurements. The figure shows that mass fraction calibration measurements satisfy the linear criteria for mass fractions less than approximately 0.8 . Similarly, the path length calibration measurements follow the linear Beer-Lambert-Bougher law for absorption thicknesses $(l)$ less than approximately $1.3 \mathrm{~mm}$. Overall, approximately $78 \%$ of the 936 calibration measurements fall within the linear criteria and none of the measurements exceeded an absorbance of 0.064 . 


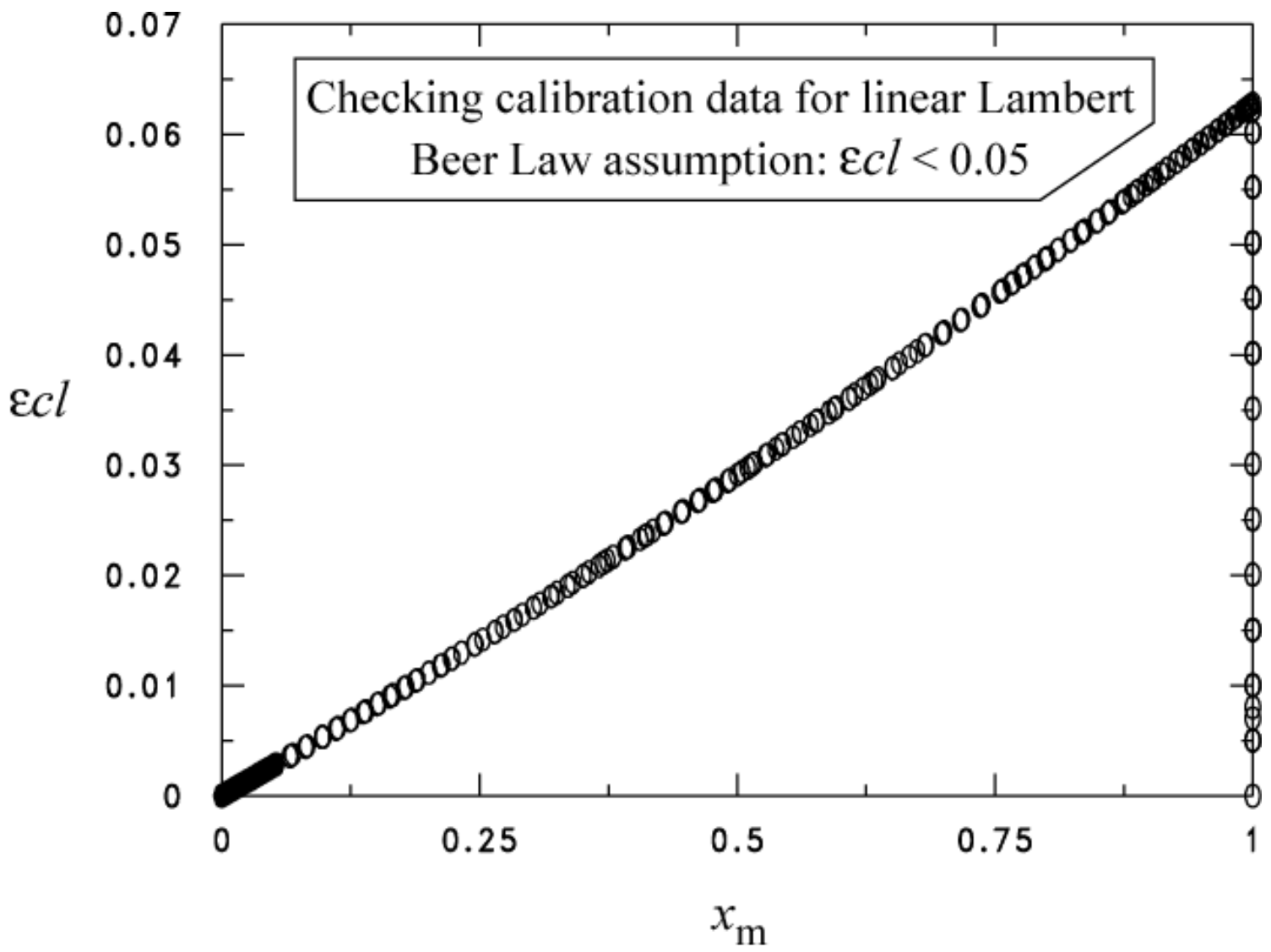

Fig. F.1 Absorbance of diesel for calibration measurements as a function of mass fraction 


\section{APPENDIX G: UNCERTAINTIES}

Figure G.1 shows the relative (percent) uncertainty of the diesel excess layer thickness $\left(U_{l e}\right)$ as a function of $l_{\mathrm{e}}$ for a bulk mass fraction of nominally $0.2 \%$. Roughly $80 \%$ of the $l_{\mathrm{e}}$ measurements for the $0.2 \%$ bulk mass fraction have a relative uncertainty of less than $25 \%$. For measurements with an relative uncertainties less than $25 \%$, the average uncertainty of $l_{\mathrm{e}}$ is approximately $\pm 6 \%$ of $l_{\mathrm{e}}$. Overall, the average uncertainty of $l_{\mathrm{e}}$ on an absolute basis was approximately $\pm 0.1 \mu \mathrm{m}$.

Similarly, Fig. G.2 shows the relative (percent) uncertainty of the diesel excess layer thickness $\left(U_{l e}\right)$ as a function of $l_{\mathrm{e}}$ for a bulk mass fraction of nominally $0.3 \%$. Roughly $92 \%$ of the $l_{\mathrm{e}}$ measurements have a relative uncertainty of less than $25 \%$. For these measurements the average uncertainty of $l_{\mathrm{e}}$ is approximately $\pm 8 \%$ of $l_{\mathrm{e}}$. Overall, the average uncertainty of $l_{\mathrm{e}}$ for the measurements with the $0.3 \%$ bulk mass fraction was approximately $\pm 0.4 \mu \mathrm{m}$.

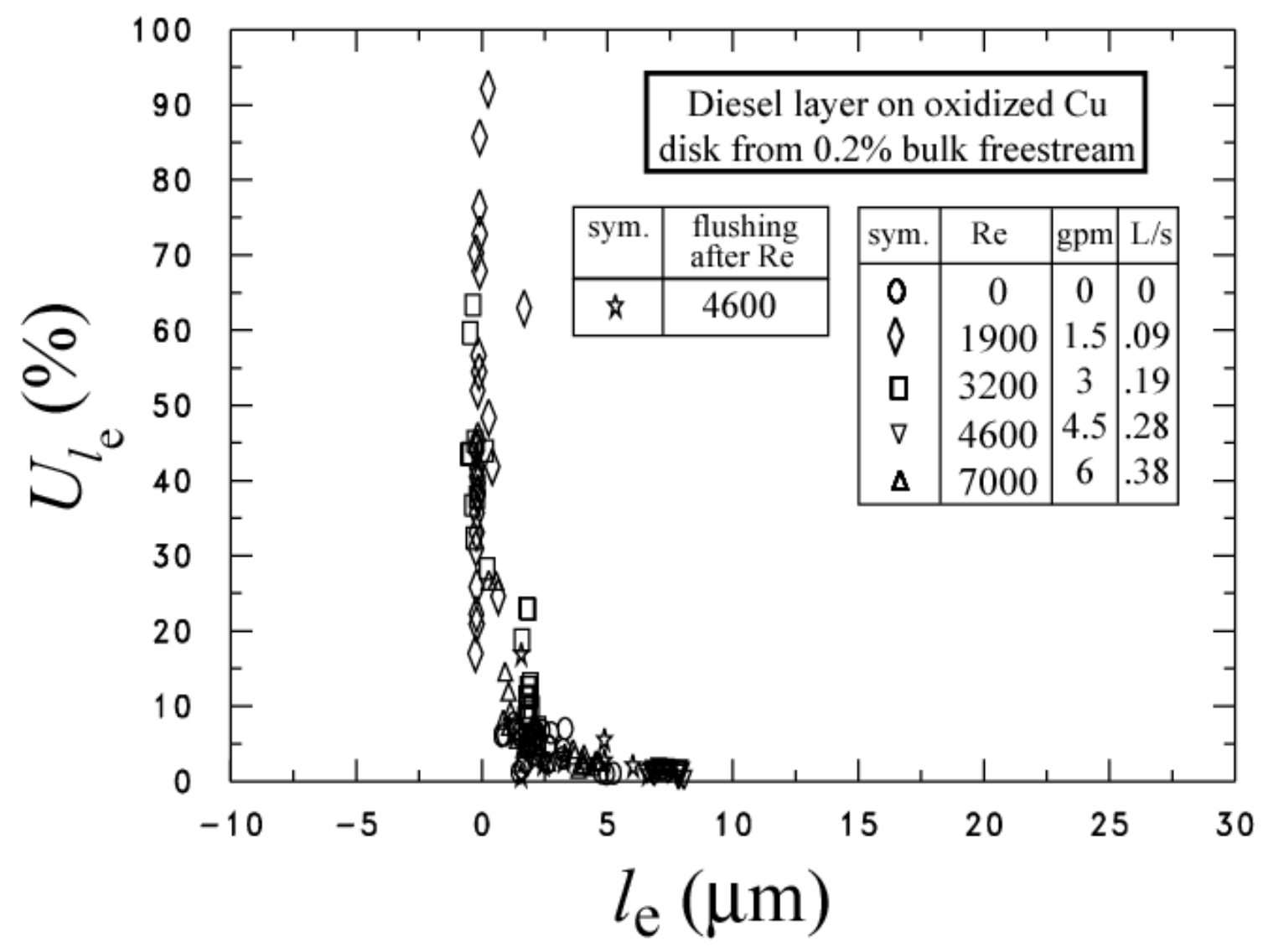

Fig. G.1 Relative uncertainty of $l_{\mathrm{e}}$ for $95 \%$ confidence level and $x_{\mathrm{b}}=0.2 \%$ 


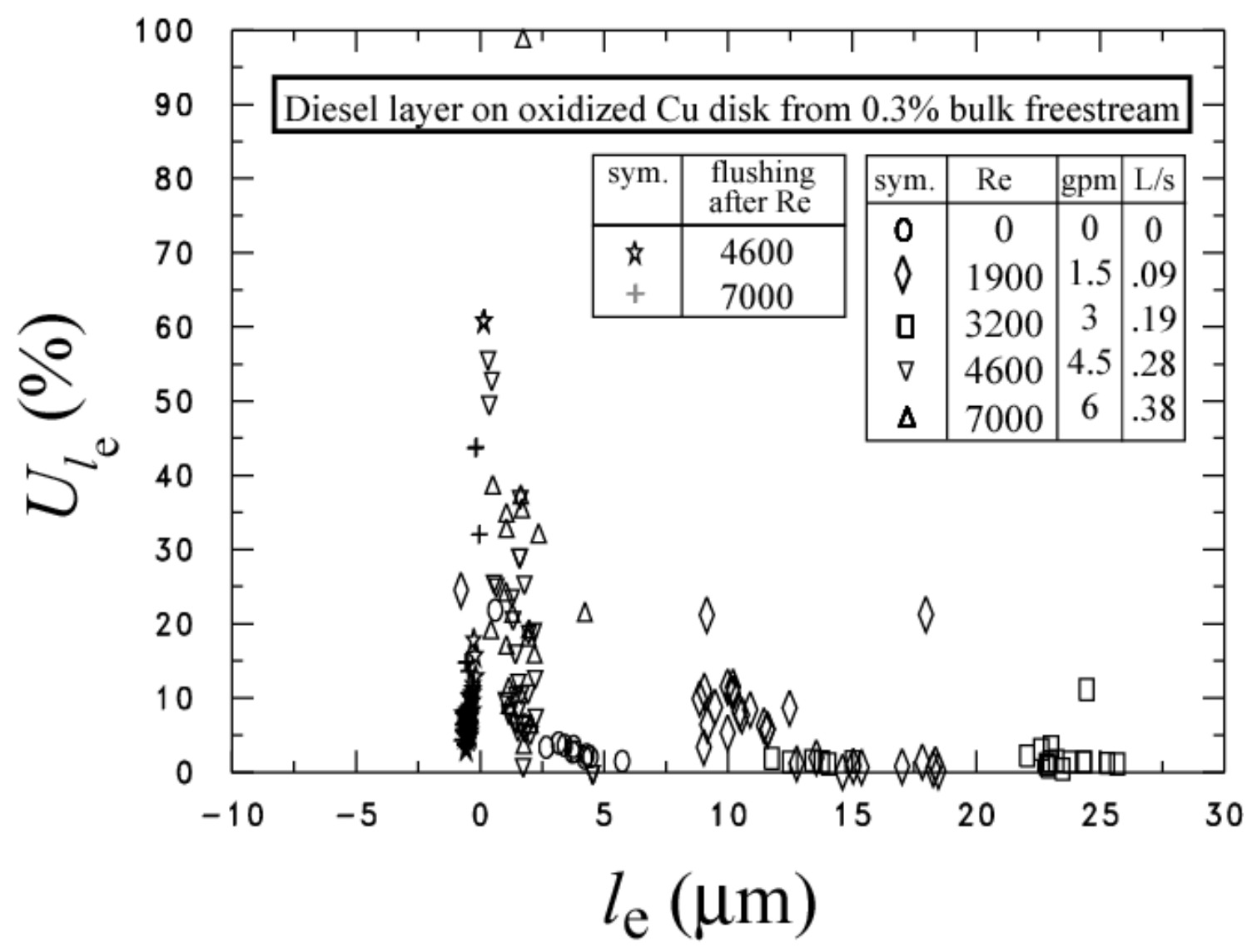

Fig. G.2 Relative uncertainty of $l_{\mathrm{e}}$ for $95 \%$ confidence level and $x_{\mathrm{b}}=0.3 \%$ 


\section{APPENDIX H: TABULATED MEASUREMENTS}

This appendix provides both raw and reduced tabulated traverse measurements. The raw measurements for the fluorescent intensities used in eq. (2), the salient measured temperatures, the varied path length $(l)$, the exposure time and the water/diesel mixture flow rate are presented in Tables H.1. The data is presented sequentially as blocks of traverse measurements (traverse of typically 13 measurements of $l$, and intensity while maintaining the flow rate and temperatures) which typically required approximately 10 min to complete. Each block or group of measurements was used to obtain a single value for the diesel excess surface density following the procedure illustrated in Fig. 7.

Reduced measurements including the excess layer thickness following the procedure demonstrated in Fig. 7 are given in Tables H.2. In addition, the excess surface density as calculated from eq. (1), the diesel mass fraction as calculated from eq. (9), the average test section fluid temperature, and the effect of temperature on fluorescence as calculated from eq. (D.1) are provided. The exposure time is the real time measured from the time starting when the clean surface was first exposed to the particular flow rate.

All tables present only a fraction of the measures and are given to provide only an example. Complete data files are available upon request.

Table H.1.1 Diesel contamination on oxidized copper surface for $\mathrm{Re}=0$ and $x_{\mathrm{b}}=0.2 \%$

\begin{tabular}{|c|c|c|c|c|c|c|c|c|c|c|c|}
\hline \multicolumn{12}{|c|}{ Diesel contamination on oxidized copper surface for $\mathrm{Re}=0$ and $x_{\mathrm{b}}=0.2 \%$ (file: trv0con $1 . t b l$ ) } \\
\hline $\begin{array}{c}F \\
(\mathrm{v})\end{array}$ & $\begin{array}{c}F_{\mathrm{r}} \\
(\mathrm{v})\end{array}$ & $\begin{array}{c}F_{100} \\
(\mathrm{v})\end{array}$ & $\begin{array}{l}F_{0} \\
(\mathrm{v})\end{array}$ & $\begin{array}{l}T_{\mathrm{Ti}} \\
(\mathrm{K})\end{array}$ & $\begin{array}{l}T_{\mathrm{To}} \\
(\mathrm{K})\end{array}$ & $\begin{array}{c}T_{\mathrm{a}} \\
(\mathrm{K})\end{array}$ & $\begin{array}{c}T_{\mathrm{b}} \\
(\mathrm{K})\end{array}$ & $\begin{array}{c}l \\
(\mathrm{~mm} \\
)\end{array}$ & $\begin{array}{l}\text { Exposu } \\
\text { re time } \\
\text { (s) }\end{array}$ & $\begin{array}{c}\text { Turbine } \\
\text { meter } \\
\dot{m}_{\mathrm{w}}(\mathrm{kg} / \mathrm{s} \\
\text { ) }\end{array}$ & $\begin{array}{c}\text { Doppler } \\
\text { meter } \\
\dot{m}_{\mathrm{w}}(\mathrm{kg} / \mathrm{s} \\
)\end{array}$ \\
\hline 0.001242 & 0.001180 & 0.942909 & 0.000008 & 294.3 & 294.4 & 297.1 & 293.6 & 1.59 & 792. & 0.0000 & 0.0625 \\
\hline 0.001207 & 0.001147 & 0.942909 & 0.000008 & 294.3 & 294.5 & 296.7 & 293.6 & 1.52 & 842. & 0.0000 & 0.0625 \\
\hline 0.001249 & 0.001187 & 0.942909 & 0.000008 & 294.4 & 294.5 & 297.1 & 293.6 & 1.40 & 894. & 0.0000 & 0.0625 \\
\hline 0.001400 & 0.001330 & 0.942909 & 0.000008 & 294.4 & 294.6 & 297.1 & 293.6 & 1.27 & 945. & 0.0000 & 0.0625 \\
\hline 0.001449 & 0.001376 & 0.942909 & 0.000008 & 294.4 & 294.6 & 296.9 & 293.6 & 1.14 & 995. & 0.0000 & 0.0625 \\
\hline 0.001484 & 0.001409 & 0.942909 & 0.000008 & 294.5 & 294.7 & 297.2 & 293.6 & 1.02 & 1052. & 0.0000 & 0.0625 \\
\hline 0.001590 & 0.001510 & 0.942909 & 0.000008 & 294.5 & 294.7 & 296.9 & 293.6 & 0.89 & 1104. & 0.0000 & 0.0625 \\
\hline 0.001641 & 0.001558 & 0.942909 & 0.000008 & 294.5 & 294.7 & 296.9 & 293.6 & 0.76 & 1156. & 0.0000 & 0.0625 \\
\hline 0.001687 & 0.001602 & 0.942909 & 0.000008 & 294.6 & 294.8 & 297.0 & 293.6 & 0.64 & 1216. & 0.0000 & 0.0697 \\
\hline 0.001770 & 0.001679 & 0.942909 & 0.000008 & 294.6 & 294.8 & 296.8 & 293.6 & 0.51 & 1268. & 0.0000 & 0.0738 \\
\hline 0.001816 & 0.001724 & 0.942909 & 0.000008 & 294.6 & 294.9 & 297.1 & 293.6 & 0.38 & 1322. & 0.0000 & 0.0697 \\
\hline 0.001890 & 0.001794 & 0.942909 & 0.000008 & 294.6 & 294.9 & 297.0 & 293.6 & 0.25 & 1377. & 0.0000 & 0.0800 \\
\hline 0.001855 & 0.001760 & 0.942909 & 0.000008 & 294.7 & 294.9 & 296.9 & 293.6 & 0.13 & 1429. & 0.0000 & 0.0853 \\
\hline 0.002019 & 0.001910 & 0.935436 & 0.000017 & 294.8 & 295.1 & 297.1 & 293.6 & 1.59 & 1620. & 0.0000 & 0.0625 \\
\hline 0.001928 & 0.001825 & 0.935436 & 0.000017 & 294.8 & 295.1 & 297.0 & 293.6 & 1.52 & 1672. & 0.0000 & 0.0624 \\
\hline 0.002032 & 0.001922 & 0.935436 & 0.000017 & 294.8 & 295.1 & 297.3 & 293.6 & 1.40 & 1726. & 0.0000 & 0.0625 \\
\hline 0.002071 & 0.001959 & 0.935436 & 0.000017 & 294.8 & 295.1 & 297.0 & 293.6 & 1.27 & 1786. & 0.0000 & 0.0625 \\
\hline 0.002037 & 0.001927 & 0.935436 & 0.000017 & 294.8 & 295.1 & 297.1 & 293.6 & 1.14 & 1838. & 0.0000 & 0.0624 \\
\hline 0.002120 & 0.002005 & 0.935436 & 0.000017 & 294.9 & 295.2 & 297.0 & 293.6 & 1.02 & 1895. & 0.0000 & 0.0625 \\
\hline 0.002135 & 0.002019 & 0.935436 & 0.000017 & 294.9 & 295.2 & 297.1 & 293.6 & 0.89 & 1948. & 0.0000 & 0.0625 \\
\hline 0.002159 & 0.002042 & 0.935436 & 0.000017 & 294.9 & 295.2 & 297.3 & 293.6 & 0.76 & 2017. & 0.0000 & 0.0739 \\
\hline 0.002213 & 0.002093 & 0.935436 & 0.000017 & 294.9 & 295.2 & 297.0 & 293.6 & 0.64 & 2072. & 0.0000 & 0.0781 \\
\hline 0.002188 & 0.002069 & 0.935436 & 0.000017 & 294.9 & 295.3 & 297.2 & 293.6 & 0.51 & 2124. & 0.0000 & 0.0745 \\
\hline 0.002277 & 0.002152 & 0.935436 & 0.000017 & 295.0 & 295.3 & 297.2 & 293.6 & 0.38 & 2177. & 0.0000 & 0.0625 \\
\hline 0.002300 & 0.002175 & 0.935436 & 0.000017 & 295.0 & 295.3 & 297.1 & 293.6 & 0.25 & 2231. & 0.0000 & 0.0625 \\
\hline 0.002257 & 0.002134 & 0.935436 & 0.000017 & 295.0 & 295.3 & 297.0 & 293.6 & 0.13 & 2283. & 0.0000 & 0.0709 \\
\hline 0.001635 & 0.001631 & 0.985852 & 0.000013 & 295.5 & 296.0 & 297.2 & 293.6 & 1.59 & 6047. & 0.0000 & 0.0625 \\
\hline 0.001660 & 0.001655 & 0.985852 & 0.000013 & 295.5 & 296.0 & 296.9 & 293.6 & 1.52 & 6098. & 0.0000 & 0.0624 \\
\hline 0.001684 & 0.001679 & 0.985852 & 0.000013 & 295.5 & 296.0 & 297.0 & 293.6 & 1.40 & 6150. & 0.0000 & 0.0625 \\
\hline
\end{tabular}


Table H.1.2 Diesel contamination on oxidized copper surface for $\mathrm{Re}=1900$ and $x_{\mathrm{b}}=0.2 \%$

\begin{tabular}{|c|c|c|c|c|c|c|c|c|c|c|c|}
\hline \multicolumn{12}{|c|}{ Diesel contamination on oxidized copper surface for $\mathrm{Re}=1900$ and $x_{\mathrm{b}}=0.2 \%$ (file: trv15con1.tbl) } \\
\hline $\begin{array}{c}F \\
(\mathrm{v})\end{array}$ & $\begin{array}{l}F_{\mathrm{r}} \\
(\mathrm{v})\end{array}$ & $\begin{array}{c}F_{100} \\
(\mathrm{v})\end{array}$ & $\begin{array}{l}F_{0} \\
(\mathrm{v})\end{array}$ & $\begin{array}{l}T_{\mathrm{Ti}} \\
(\mathrm{K})\end{array}$ & $\begin{array}{l}T_{\mathrm{To}} \\
(\mathrm{K})\end{array}$ & $\begin{array}{c}T_{\mathrm{a}} \\
(\mathrm{K})\end{array}$ & $\begin{array}{c}T_{\mathrm{b}} \\
(\mathrm{K})\end{array}$ & $\begin{array}{c}l \\
(\mathrm{~mm})\end{array}$ & $\begin{array}{l}\text { Exposure } \\
\text { time (s) }\end{array}$ & $\begin{array}{l}\text { Turbine } \\
\text { meter } \\
\dot{m}_{\mathrm{w}}(\mathrm{kg} / \mathrm{s}\end{array}$ & $\begin{array}{l}\text { Doppler } \\
\text { meter } \\
\dot{m}_{\mathrm{w}}(\mathrm{kg} / \mathrm{s}\end{array}$ \\
\hline 0.003617 & 0.003772 & 1.010201 & 0.000107 & 295.5 & 295.5 & 297.6 & 293.6 & 1.59 & 1008. & 0.0763 & 0.1093 \\
\hline 0.003411 & 0.003563 & 1.010201 & 0.000107 & 295.5 & 295.4 & 297.7 & 293.6 & 1.52 & 1065. & 0.0737 & 0.1058 \\
\hline 0.003222 & 0.003372 & 1.010201 & 0.000107 & 295.4 & 295.4 & 297.5 & 293.6 & 1.40 & 1135. & 0.0759 & 0.1042 \\
\hline 0.003004 & 0.003151 & 1.010201 & 0.000107 & 295.4 & 295.4 & 297.4 & 293.6 & $\begin{array}{l}1.40 \\
1.27\end{array}$ & 1179. & 0.0742 & 0.1106 \\
\hline 0.002763 & 0.002906 & 1.010201 & 0.000107 & 295.4 & 295.4 & 297.8 & 293.6 & 1.14 & 1258. & 0.0750 & 0.1004 \\
\hline 0.002261 & 0.002398 & 1.010201 & 0.000107 & 295.4 & 295.4 & 297.6 & 293.6 & $\begin{array}{l}1.14 \\
1.02\end{array}$ & 1321. & 0.0755 & 0.1085 \\
\hline 0.002154 & 0.002289 & 1.010201 & 0.000107 & 295.3 & 295.3 & 297.4 & 293.6 & $\begin{array}{l}1.02 \\
0.89\end{array}$ & 1392. & 0.0712 & 0.1106 \\
\hline 0.001791 & 0.001921 & $\begin{array}{l}1.010201 \\
1.010201\end{array}$ & 0.000107 & 295.3 & 295.3 & 297.6 & 293.6 & 0.76 & 1492. & 0.0732 & 0.1222 \\
\hline 0.001876 & 0.002007 & 1.010201 & 0.000107 & 295.3 & 295.3 & 297.5 & 293.6 & 0.64 & 1536. & 0.0742 & 0.1033 \\
\hline 0.001588 & 0.001716 & 1.010201 & 0.000107 & 295.2 & 295.3 & 297.6 & 293.6 & 0.51 & 1574. & 0.0745 & 0.1069 \\
\hline 0.001351 & 0.001475 & $\begin{array}{l}1.010201 \\
1.010201\end{array}$ & 0.000107 & 295.2 & 295.2 & 297.5 & 293.6 & 0.38 & 1617. & 0.0710 & 0.1023 \\
\hline 0.000918 & 0.001038 & 1.010201 & 0.000107 & 295.2 & 295.2 & 297.5 & 293.6 & 0.25 & 1661. & 0.0720 & 0.1124 \\
\hline 0.000574 & 0.000688 & 1.010201 & 0.000107 & 295.2 & 295.2 & 297.6 & 293.6 & 0.13 & 1705. & 0.0747 & 0.1087 \\
\hline 0.002523 & 0.002562 & $\begin{array}{l}1.010 \angle 01 \\
1.008425\end{array}$ & 0.000018 & 293.5 & 293.5 & 297.6 & 293.6 & $\begin{array}{l}0.15 \\
1.59\end{array}$ & 61523. & 0.0844 & 0.1042 \\
\hline 0.002449 & 0.002488 & 1.008425 & 0.000018 & 293.5 & 293.5 & 297.2 & 293.6 & 1.52 & 61568. & 0.0874 & 0.1111 \\
\hline 0.002293 & 0.002330 & 1.008425 & 0.000018 & 293.5 & 293.5 & 297.6 & 293.6 & $\begin{array}{l}1.02 \\
1.40\end{array}$ & 61614. & 0.0864 & 0.1151 \\
\hline 0.002095 & 0.002131 & 1.008425 & 0.000018 & 293.4 & 293.4 & 297.5 & 293.6 & $\begin{array}{l}1.40 \\
1.27\end{array}$ & 61656. & 0.0869 & 0.1167 \\
\hline 0.001901 & 0.001935 & 1.008425 & 0.000018 & 293.4 & 293.4 & 297.6 & 293.6 & 1.14 & 61697. & 0.0870 & 0.1146 \\
\hline 0.001768 & 0.001801 & 1.008425 & 0.000018 & 293.4 & 293.4 & 297.3 & 293.6 & $\begin{array}{l}1.44 \\
1.02\end{array}$ & 61743. & 0.0877 & 0.1175 \\
\hline 0.001607 & 0.001638 & 1.008425 & 0.000018 & 293.4 & 293.4 & 297.3 & 293.6 & $\begin{array}{l}1.02 \\
0.89\end{array}$ & 61782. & 0.0833 & 0.1163 \\
\hline 0.001453 & 0.001483 & 1.008425 & 0.000018 & 293.4 & 293.4 & 297.4 & 293.6 & 0.76 & 61823. & 0.0866 & 0.1192 \\
\hline 0.001238 & 0.001266 & 1.008425 & 0.000018 & 293.4 & 293.4 & 297.2 & 293.6 & 0.64 & 61865. & 0.0853 & 0.1168 \\
\hline 0.001073 & 0.001100 & 1.008425 & 0.000018 & 293.3 & 293.4 & 297.1 & 293.6 & 0.51 & 61905. & 0.0827 & 0.1161 \\
\hline 0.000963 & 0.000989 & 1.008425 & 0.000018 & 293.3 & 293.3 & 297.4 & 293.6 & 0.38 & 61946. & 0.0866 & 0.1187 \\
\hline 0.000763 & 0.000788 & 1.008425 & 0.000018 & 293.3 & 293.3 & 297.1 & 293.6 & 0.25 & 61987. & 0.0858 & 0.1168 \\
\hline 0.000609 & 0.000632 & 1.008425 & 0.000018 & 293.3 & 293.3 & 297.3 & 293.6 & 0.13 & 62026. & 0.0875 & 0.1157 \\
\hline 0.002626 & 0.002759 & 1.007386 & 0.000116 & 293.3 & 293.3 & 297.5 & 293.6 & $\begin{array}{l}0.15 \\
1.59\end{array}$ & 62198. & 0.0833 & 0.1161 \\
\hline 0.002493 & 0.002625 & 1.007386 & 0.000116 & 293.3 & 293.3 & 297.7 & 293.6 & $\begin{array}{l}1.05 \\
1.52\end{array}$ & 62241. & 0.0848 & 0.1130 \\
\hline 0.002345 & 0.002476 & 1.007386 & 0.000116 & 293.2 & 293.2 & 297.5 & 293.6 & $\begin{array}{l}1.02 \\
1.40\end{array}$ & 62282. & 0.0870 & 0.1108 \\
\hline 0.002184 & 0.002314 & 1.007386 & 0.000116 & 293.2 & 293.2 & 297.3 & 293.6 & $\begin{array}{l}1.40 \\
1.27\end{array}$ & 62324. & 0.0853 & 0.1185 \\
\hline 0.002013 & $\begin{array}{l}0.002014 \\
0.002142\end{array}$ & 1.007386 & 0.000116 & 293.2 & 293.2 & 297.2 & 293.6 & 1.14 & 62367. & 0.0833 & 0.1127 \\
\hline 0.001872 & 0.002001 & 1.007386 & 0.000116 & 293.2 & 293.2 & 296.9 & 293.6 & $\begin{array}{l}1.14 \\
1.02\end{array}$ & 62409. & 0.0872 & 0.1105 \\
\hline 0.001720 & 0.001847 & 1.007386 & 0.000116 & 293.2 & 293.2 & 297.0 & 293.6 & $\begin{array}{l}1.02 \\
0.89\end{array}$ & 62454. & 0.0852 & 0.1062 \\
\hline 0.001540 & 0.001666 & 1.007386 & 0.000116 & 293.2 & 293.2 & 297.3 & 293.6 & 0.76 & 62498. & $\begin{array}{l}0.0052 \\
0.0858\end{array}$ & 0.1119 \\
\hline 0.001507 & 0.001633 & 1.007386 & 0.000116 & 293.2 & 293.2 & 297.3 & 293.6 & 0.64 & 62539. & 0.0829 & 0.1194 \\
\hline 0.001393 & 0.001518 & 1.007386 & 0.000116 & 293.2 & 293.2 & 297.1 & 293.6 & 0.51 & 62581. & 0.0862 & 0.1241 \\
\hline 0.001258 & 0.001382 & 1.007386 & 0.000116 & 293.2 & 293.2 & 297.2 & 293.6 & 0.38 & 62622. & 0.0863 & $\begin{array}{l}0.1241 \\
0.1229\end{array}$ \\
\hline 0.001078 & 0.001201 & 1.007386 & 0.000116 & 293.2 & 293.2 & 297.1 & 293.6 & 0.25 & 62664. & 0.0868 & 0.1238 \\
\hline 0.000830 & 0.000951 & 1.007386 & 0.000116 & 293.2 & 293.2 & 297.2 & 293.6 & 0.13 & 62707. & 0.0843 & 0.1239 \\
\hline 0.002796 & 0.002894 & 1.012094 & 0.000064 & 293.7 & 293.7 & 297.5 & 293.6 & $\begin{array}{l}0.15 \\
1.59\end{array}$ & 66387. & 0.0870 & 0.1184 \\
\hline 0.002666 & 0.002762 & 1.012094 & 0.000064 & 293.7 & 293.7 & 297.6 & 293.6 & $\begin{array}{l}1.05 \\
1.52\end{array}$ & 66427. & 0.0862 & 0.1222 \\
\hline 0.002592 & 0.002687 & 1.012094 & 0.000064 & 293.6 & 293.6 & 297.5 & 293.6 & $\begin{array}{l}1.02 \\
1.40\end{array}$ & 66469. & 0.0845 & 0.1167 \\
\hline 0.002529 & 0.002624 & 1.012094 & 0.000064 & 293.6 & 293.6 & 297.6 & 293.6 & $\begin{array}{l}1.40 \\
1.27\end{array}$ & 66509. & 0.0883 & 0.1163 \\
\hline 0.003842 & 0.003952 & 1.012094 & 0.000064 & 293.6 & 293.6 & 297.4 & 293.6 & 1.14 & 66551. & 0.0877 & 0.1179 \\
\hline 0.003831 & 0.003941 & 1.012094 & 0.000064 & 293.6 & 293.6 & 297.2 & 293.6 & $\begin{array}{l}1.14 \\
1.02\end{array}$ & 66592. & 0.0877 & 0.1143 \\
\hline 0.003148 & 0.003250 & 1.012094 & 0.000064 & 293.5 & 293.6 & 297.4 & 293.6 & $\begin{array}{l}1.02 \\
0.89\end{array}$ & 66632. & 0.0865 & 0.1092 \\
\hline 0.002608 & 0.002703 & 1.012094 & 0.000064 & 293.5 & 293.5 & 297.4 & 293.6 & 0.76 & 66709. & 0.0816 & 0.1067 \\
\hline 0.002231 & 0.002321 & 1.012094 & 0.000064 & 293.5 & 293.5 & 297.5 & 293.6 & 0.64 & 66749. & 0.0836 & 0.1163 \\
\hline 0.001971 & 0.002059 & 1.012094 & 0.000064 & 293.4 & 293.5 & 297.7 & 293.6 & 0.51 & 66788. & 0.0864 & 0.1092 \\
\hline 0.001619 & 0.001702 & 1.012094 & 0.000064 & 293.4 & 293.4 & 297.5 & 293.6 & 0.38 & 66829. & 0.0829 & 0.1110 \\
\hline 0.001524 & 0.001606 & 1.012094 & 0.000064 & 293.4 & 293.4 & 297.2 & 293.6 & 0.25 & 66869. & 0.0872 & 0.1138 \\
\hline 0.001461 & 0.001542 & 1.012094 & 0.000064 & 293.4 & 293.4 & 297.1 & 293.6 & 0.13 & 66910. & 0.0850 & 0.1169 \\
\hline 0.003188 & 0.003266 & 1.009252 & 0.000047 & 294.0 & 293.9 & 296.5 & 293.6 & $\begin{array}{l}0.10 \\
1.59\end{array}$ & 69847. & 0.0853 & 0.1064 \\
\hline 0.003181 & 0.003259 & $\begin{array}{l}1.000252 \\
1.009252\end{array}$ & 0.000047 & 294.0 & 293.9 & 296.3 & 293.6 & $\begin{array}{l}1.03 \\
1.52\end{array}$ & 69884. & 0.0834 & 0.1082 \\
\hline 0.003045 & 0.003122 & 1.009252 & 0.000047 & 294.0 & 293.9 & 296.5 & 293.6 & $\begin{array}{l}1.02 \\
1.40\end{array}$ & 69926. & 0.0857 & 0.1098 \\
\hline 0.002962 & 0.003038 & 1.009252 & 0.000047 & 294.0 & 294.0 & 296.5 & 293.6 & 1.27 & 69967. & 0.0859 & 0.1106 \\
\hline 0.002824 & 0.002898 & $\begin{array}{l}1.000252 \\
1.009252\end{array}$ & 0.000047 & 294.0 & 294.0 & 296.3 & 293.6 & 1.14 & 70009 . & 0.0862 & 0.1147 \\
\hline
\end{tabular}


Table H.1.3 Diesel contamination on oxidized copper surface for $\operatorname{Re}=3200$ and $x_{\mathrm{b}}=0.2 \%$

\begin{tabular}{|c|c|c|c|c|c|c|c|c|c|c|c|}
\hline \multicolumn{12}{|c|}{ Diesel contamination on oxidized copper surface for $\mathrm{Re}=3200$ and $x_{\mathrm{b}}=0.2 \%$ (file: trv3con1.tbl) } \\
\hline $\begin{array}{c}F \\
(\mathrm{v})\end{array}$ & $\begin{array}{l}F_{\mathrm{r}} \\
(\mathrm{v})\end{array}$ & $\begin{array}{c}F_{100} \\
(\mathrm{v})\end{array}$ & $\begin{array}{l}F_{0} \\
(\mathrm{v})\end{array}$ & $\begin{array}{l}T_{\mathrm{Ti}} \\
(\mathrm{K})\end{array}$ & $\begin{array}{l}T_{\mathrm{To}} \\
(\mathrm{K})\end{array}$ & $\begin{array}{c}T_{\mathrm{a}} \\
(\mathrm{K})\end{array}$ & $\begin{array}{c}T_{\mathrm{b}} \\
(\mathrm{K})\end{array}$ & $\begin{array}{c}l \\
(\mathrm{~mm})\end{array}$ & $\begin{array}{c}\text { Exposure } \\
\text { time (s) }\end{array}$ & $\begin{array}{c}\text { Turbine } \\
\text { meter } \\
\dot{m}_{\mathrm{w}}(\mathrm{kg} / \mathrm{s})\end{array}$ & $\begin{array}{c}\text { Doppler } \\
\text { meter } \\
\dot{m}_{\mathrm{w}}(\mathrm{kg} / \mathrm{s})\end{array}$ \\
\hline 0.003391 & 0.003385 & 1.002145 & -0.0000260 & 296.2 & 296.1 & 296.1 & 293.6 & 1.59 & 1521. & 0.1558 & 0.1596 \\
\hline 0.003188 & 0.003182 & 1.002145 & -0.000026 & 296.2 & 296.1 & 296.0 & 293.6 & 1.52 & 1563. & 0.1512 & 0.1566 \\
\hline 0.003292 & 0.003286 & 1.002145 & -0.000026 & 296.2 & 296.1 & 296.0 & 293.6 & 1.40 & 1607. & 0.1550 & 0.1613 \\
\hline 0.003003 & 0.002996 & 1.002145 & -0.000026 & 296.2 & 296.2 & 296.0 & 293.6 & 1.27 & 1706. & 0.1531 & 0.1577 \\
\hline 0.002738 & 0.002728 & 1.002145 & -0.000026 & 296.2 & 296.2 & 295.9 & 293.6 & 1.14 & 1749. & 0.1509 & 0.1601 \\
\hline 0.002347 & 0.002335 & 1.002145 & -0.000026 & 296.2 & 296.2 & 295.7 & 293.6 & 1.02 & 1789. & 0.1476 & 0.1620 \\
\hline 0.001922 & 0.001908 & 1.002145 & -0.000026 & 296.2 & 296.2 & 295.3 & 293.6 & 0.89 & 1831. & 0.1550 & 0.1634 \\
\hline 0.001679 & 0.001663 & 1.002145 & -0.000026 & 296.2 & 296.2 & 296.5 & 293.6 & 0.76 & 1873. & 0.1453 & 0.1589 \\
\hline 0.001387 & 0.001369 & 1.002145 & -0.000026 & 296.2 & 296.2 & 296.2 & 293.6 & 0.64 & 1920. & 0.1557 & 0.1587 \\
\hline 0.001198 & 0.001179 & 1.002145 & -0.000026 & 296.2 & 296.2 & 295.6 & 293.6 & 0.51 & 1966. & 0.1535 & 0.1517 \\
\hline 0.000929 & 0.000908 & 1.002145 & -0.000026 & 296.2 & 296.2 & 295.8 & 293.6 & 0.38 & 2009. & 0.1550 & 0.1509 \\
\hline 0.000584 & 0.000561 & 1.002145 & -0.000026 & 296.2 & 296.2 & 296.1 & 293.6 & 0.25 & 2051. & 0.1542 & 0.1502 \\
\hline 0.000082 & 0.000056 & 1.002145 & -0.000026 & 296.2 & 296.2 & 296.0 & 293.6 & 0.13 & 2092. & 0.1501 & 0.1581 \\
\hline 0.003134 & 0.003115 & 1.006735 & -0.000052 & 296.2 & 296.2 & 295.3 & 293.6 & 1.59 & 2224. & 0.1470 & 0.1501 \\
\hline 0.002885 & 0.002864 & 1.006735 & -0.000052 & 296.2 & 296.2 & 295.2 & 293.6 & 1.52 & 2267. & 0.1509 & 0.1669 \\
\hline 0.002841 & 0.002819 & 1.006735 & -0.000052 & 296.2 & 296.1 & 295.4 & 293.7 & 1.40 & 2310. & 0.1550 & 0.1654 \\
\hline 0.002923 & 0.002902 & 1.006735 & -0.000052 & 296.2 & 296.1 & 295.9 & 293.6 & 1.27 & 2354. & 0.1535 & 0.1563 \\
\hline 0.002583 & 0.002558 & 1.006735 & -0.000052 & 296.1 & 296.1 & 295.5 & 293.6 & 1.14 & 2401. & 0.1480 & 0.1649 \\
\hline 0.002263 & 0.002235 & 1.006735 & -0.000052 & 296.1 & 296.1 & 296.0 & 293.6 & 1.02 & 2442. & 0.1498 & 0.1600 \\
\hline 0.001828 & 0.001795 & 1.006735 & -0.000052 & 296.1 & 296.1 & 295.8 & 293.6 & 0.89 & 2482. & 0.1546 & 0.1587 \\
\hline 0.001410 & 0.001373 & 1.006735 & -0.000052 & 296.1 & 296.1 & 295.4 & 293.6 & 0.76 & 2524. & 0.1523 & 0.1517 \\
\hline 0.001121 & 0.001081 & 1.006735 & -0.000052 & 296.1 & 296.1 & 296.1 & 293.6 & 0.64 & 2569. & 0.1516 & 0.1553 \\
\hline 0.000973 & 0.000931 & 1.006735 & -0.000052 & 296.1 & 296.0 & 295.6 & 293.6 & 0.51 & 2775. & 0.1487 & 0.1572 \\
\hline 0.000673 & 0.000628 & 1.006735 & -0.000052 & 296.0 & 296.0 & 295.7 & 293.6 & 0.38 & 2819. & 0.1487 & 0.1486 \\
\hline 0.000397 & 0.000349 & 1.006735 & -0.000052 & 296.0 & 296.0 & 295.8 & 293.6 & 0.25 & 2862. & 0.1531 & 0.1543 \\
\hline 0.000080 & 0.000029 & 1.006735 & -0.000052 & 296.0 & 296.0 & 295.8 & 293.6 & 0.13 & 2903. & 0.1466 & 0.1421 \\
\hline 0.002741 & 0.002780 & 1.007206 & 0.000016 & 294.4 & 294.4 & 296.2 & 293.6 & 1.59 & 5441. & 0.1543 & 0.1350 \\
\hline 0.002670 & 0.002709 & 1.007206 & 0.000016 & 294.4 & 294.4 & 296.6 & 293.6 & 1.52 & 5486. & 0.1535 & 0.1459 \\
\hline 0.002594 & 0.002632 & 1.007206 & 0.000016 & 294.4 & 294.3 & 296.6 & 293.6 & 1.40 & 5531. & 0.1550 & 0.1662 \\
\hline 0.002476 & 0.002512 & 1.007206 & 0.000016 & 294.3 & 294.3 & 296.6 & 293.6 & 1.27 & 5572. & 0.1562 & 0.1562 \\
\hline 0.002236 & 0.002270 & 1.007206 & 0.000016 & 294.3 & 294.3 & 296.5 & 293.6 & 1.14 & 5619. & 0.1509 & 0.1455 \\
\hline 0.001912 & 0.001944 & 1.007206 & 0.000016 & 294.3 & 294.3 & 296.8 & 293.6 & 1.02 & 5660. & 0.1532 & 0.1485 \\
\hline 0.001566 & 0.001595 & 1.007206 & 0.000016 & 294.2 & 294.2 & 296.9 & 293.6 & 0.89 & 5702. & 0.1488 & 0.1517 \\
\hline 0.001214 & 0.001240 & 1.007206 & 0.000016 & 294.2 & 294.2 & 296.4 & 293.6 & 0.76 & 5744. & 0.1528 & 0.1430 \\
\hline 0.000993 & 0.001017 & 1.007206 & 0.000016 & 294.2 & 294.2 & 296.4 & 293.6 & 0.64 & 5783. & 0.1488 & 0.1453 \\
\hline 0.000791 & 0.000813 & 1.007206 & 0.000016 & 294.1 & 294.1 & 296.0 & 293.6 & 0.51 & 5824. & 0.1477 & 0.1454 \\
\hline 0.000601 & 0.000622 & 1.007206 & 0.000016 & 294.1 & 294.1 & 296.5 & 293.6 & 0.38 & 5866. & 0.1554 & 0.1476 \\
\hline 0.000331 & 0.000350 & 1.007206 & 0.000016 & 294.1 & 294.1 & 295.8 & 293.6 & 0.25 & 5909. & 0.1539 & 0.1425 \\
\hline 0.000083 & 0.000100 & 1.007206 & 0.000016 & 294.0 & 294.0 & 295.7 & 293.6 & 0.13 & 5957. & 0.1481 & 0.1432 \\
\hline 0.002855 & 0.002877 & 0.999473 & 0.000026 & 293.2 & 293.2 & 296.4 & 293.7 & 1.59 & 8736. & 0.1562 & 0.1391 \\
\hline 0.002723 & 0.002746 & 0.999473 & 0.000026 & 293.2 & 293.2 & 296.1 & 293.7 & 1.52 & 8776. & 0.1562 & 0.1290 \\
\hline 0.002447 & 0.002470 & 0.999473 & 0.000026 & 293.2 & 293.2 & 296.3 & 293.6 & 1.40 & 8821. & 0.1570 & 0.1397 \\
\hline 0.002283 & 0.002307 & 0.999473 & 0.000026 & 293.3 & 293.2 & 296.1 & 293.6 & 1.27 & 8865. & 0.1524 & 0.1531 \\
\hline 0.002136 & 0.002160 & 0.999473 & 0.000026 & 293.3 & 293.3 & 295.9 & 293.7 & 1.14 & 8906. & 0.1502 & 0.1457 \\
\hline 0.001921 & 0.001946 & 0.999473 & 0.000026 & 293.3 & 293.3 & 295.9 & 293.6 & 1.02 & 8946. & 0.1547 & 0.1348 \\
\hline 0.001659 & 0.001683 & 0.999473 & 0.000026 & 293.3 & 293.3 & 296.0 & 293.6 & 0.89 & 8988. & 0.1513 & 0.1328 \\
\hline 0.001420 & 0.001445 & 0.999473 & 0.000026 & 293.3 & 293.3 & 296.7 & 293.6 & 0.76 & 9028. & 0.1521 & 0.1226 \\
\hline 0.001140 & 0.001165 & 0.999473 & 0.000026 & 293.3 & 293.3 & 296.6 & 293.7 & 0.64 & 9070. & 0.1528 & 0.1392 \\
\hline 0.000918 & 0.000943 & 0.999473 & 0.000026 & 293.4 & 293.3 & 296.3 & 293.6 & 0.51 & 9116. & 0.1578 & 0.1413 \\
\hline 0.000610 & 0.000636 & 0.999473 & 0.000026 & 293.4 & 293.3 & 296.2 & 293.6 & 0.38 & 9162. & 0.1547 & 0.1497 \\
\hline 0.000373 & 0.000399 & 0.999473 & 0.000026 & 293.4 & 293.4 & 296.1 & 293.6 & 0.25 & 9204. & 0.1502 & 0.1482 \\
\hline 0.000174 & 0.000200 & 0.999473 & 0.000026 & 293.4 & 293.4 & 296.0 & 293.6 & 0.13 & 9244. & 0.1506 & 0.1494 \\
\hline 0.002669 & 0.002642 & 0.986360 & 0.000010 & 293.5 & 293.5 & 296.1 & 293.7 & 1.59 & 9383. & 0.1559 & 0.1608 \\
\hline 0.002560 & 0.002534 & 0.986360 & 0.000010 & 293.5 & 293.5 & 296.7 & 293.7 & 1.52 & 9424. & 0.1510 & 0.1470 \\
\hline 0.002486 & 0.002462 & 0.986360 & 0.000010 & 293.5 & 293.5 & 296.8 & 293.6 & 1.40 & 9465. & 0.1543 & 0.1328 \\
\hline 0.002299 & 0.002278 & 0.986360 & 0.000010 & 293.5 & 293.5 & 296.8 & 293.6 & 1.27 & 9506. & 0.1543 & 0.1232 \\
\hline 0.002114 & 0.002095 & 0.986360 & 0.000010 & 293.6 & 293.5 & 296.9 & 293.6 & 1.14 & 9548. & 0.1562 & 0.1415 \\
\hline 0.001922 & 0.001905 & 0.986360 & 0.000010 & 293.6 & 293.6 & 296.5 & 293.6 & 1.02 & 9592. & 0.1558 & 0.1516 \\
\hline
\end{tabular}


Table H.1.4 Diesel contamination on oxidized copper surface for $\mathrm{Re}=4600$ and $x_{\mathrm{b}}=0.2 \%$

\begin{tabular}{|c|c|c|c|c|c|c|c|c|c|c|c|}
\hline \multicolumn{12}{|c|}{ Diesel contamination on oxidized copper surface for $\mathrm{Re}=4600$ and $x_{\mathrm{b}}=0.2 \%$ (file: trv45con1.tbl) } \\
\hline $\begin{array}{c}F \\
(\mathrm{v})\end{array}$ & $\begin{array}{l}F_{\mathrm{r}} \\
(\mathrm{v})\end{array}$ & $\begin{array}{c}F_{100} \\
(\mathrm{v})\end{array}$ & $\begin{array}{l}F_{0} \\
(\mathrm{v})\end{array}$ & $\begin{array}{c}T_{\mathrm{Ti}} \\
(\mathrm{K})\end{array}$ & $\begin{array}{l}T_{\text {To }} \\
(\mathrm{K})\end{array}$ & $\begin{array}{c}T_{\mathrm{a}} \\
(\mathrm{K})\end{array}$ & $\begin{array}{c}T_{\mathrm{b}} \\
(\mathrm{K})\end{array}$ & $\begin{array}{c}l \\
(\mathrm{~mm})\end{array}$ & $\begin{array}{c}\text { Exposure } \\
\text { time (s) }\end{array}$ & $\begin{array}{c}\text { Turbine } \\
\text { meter } \\
\dot{m}_{\mathrm{w}}(\mathrm{k} \\
\mathrm{g} / \mathrm{s})\end{array}$ & $\begin{array}{c}\text { Doppler } \\
\text { meter } \\
\dot{m}_{\mathrm{w}}(\mathrm{k} \\
\mathrm{g} / \mathrm{s})\end{array}$ \\
\hline 0.0071260 & 0.007198 & 1.006097 & 0.000032 & 293.4 & 293.4 & 294.7 & 293.6 & 1.59 & 236041. & 0.2280 & 0.2502 \\
\hline 0.007096 & 0.007168 & 1.006097 & 0.000032 & 293.4 & 293.4 & 294.5 & 293.6 & 1.52 & 236081. & 0.2305 & 0.2517 \\
\hline 0.007047 & 0.007119 & 1.006097 & 0.000032 & 293.4 & 293.4 & 294.6 & $\begin{array}{l}293.0 \\
293.6\end{array}$ & $\begin{array}{l}1.02 \\
1.40\end{array}$ & 236122 . & 0.2191 & 0.2518 \\
\hline 0.007094 & 0.007166 & 1.006097 & 0.000032 & 293.4 & 293.4 & 294.7 & 293.6 & $\begin{array}{l}1.40 \\
1.27\end{array}$ & 236166. & 0.2280 & 0.2526 \\
\hline 0.007073 & 0.007145 & 1.006097 & 0.000032 & 293.4 & 293.4 & 294.6 & 293.6 & 1.14 & 236207. & 0.2264 & 0.2534 \\
\hline 0.007054 & 0.007126 & 1.006097 & 0.000032 & 293.4 & 293.4 & 294.7 & 293.6 & $\begin{array}{l}1.14 \\
1.02\end{array}$ & 236249. & 0.2238 & 0.2539 \\
\hline 0.007076 & 0.007148 & 1.006097 & 0.000032 & 293.4 & 293.4 & 294.7 & 293.6 & 0.89 & 236293. & 0.2255 & 0.2511 \\
\hline 0.007170 & 0.007242 & 1.006097 & 0.000032 & 293.4 & 293.4 & 294.7 & 293.6 & 0.76 & 236338. & 0.2169 & 0.2425 \\
\hline 0.007069 & 0.007141 & 1.006097 & 0.000032 & 293.4 & 293.4 & 295.0 & 293.6 & 0.64 & 236382. & 0.2264 & 0.2245 \\
\hline 0.007079 & 0.007151 & 1.006097 & 0.000032 & 293.4 & 293.4 & 294.9 & 293.6 & 0.51 & 236423. & 0.2199 & 0.2101 \\
\hline 0.007225 & 0.007299 & 1.006097 & 0.000032 & 293.4 & 293.4 & 294.9 & 293.6 & 0.38 & 236464. & 0.2222 & 0.1988 \\
\hline 0.007117 & 0.007190 & 1.006097 & 0.000032 & 293.4 & 293.4 & 294.6 & 293.6 & 0.25 & 236505. & 0.2176 & 0.1980 \\
\hline 0.007247 & 0.007321 & 1.006097 & 0.000032 & 293.5 & 293.4 & 294.6 & 293.6 & 0.13 & 236554. & 0.2246 & 0.1958 \\
\hline 0.007107 & 0.007254 & 1.010847 & 0.000072 & 293.5 & $\begin{array}{l}293.4 \\
293.5\end{array}$ & 294.7 & $\begin{array}{l}293.0 \\
293.6\end{array}$ & $\begin{array}{l}0.15 \\
1.59\end{array}$ & 236711. & 0.2215 & 0.2049 \\
\hline 0.007058 & 0.007205 & 1.010847 & 0.000072 & 293.6 & 293.5 & 294.5 & 293.6 & 1.52 & 236754. & 0.2246 & 0.2016 \\
\hline 0.006926 & 0.007071 & 1.010847 & 0.000072 & 293.6 & 293.5 & 294.4 & 293.6 & 1.40 & 236797. & 0.2215 & 0.2097 \\
\hline 0.006983 & 0.007130 & $\begin{array}{l}1.010047 \\
1.010847\end{array}$ & 0.000072 & 293.6 & 293.6 & 294.6 & $\begin{array}{l}293.0 \\
293.6\end{array}$ & 1.27 & 236838. & 0.2215 & 0.2197 \\
\hline 0.007073 & 0.007221 & 1.010847 & 0.000072 & 293.6 & 293.6 & 294.7 & 293.6 & 1.14 & 236883. & 0.2223 & 0.2426 \\
\hline 0.006951 & 0.007097 & 1.010847 & 0.000072 & 293.6 & 293.6 & 294.0 & 293.6 & 1.02 & 236931. & 0.2184 & 0.2300 \\
\hline 0.007007 & 0.007155 & 1.010847 & 0.000072 & 293.6 & 293.6 & 294.4 & 293.6 & 0.89 & 236982. & 0.2176 & 0.2184 \\
\hline 0.007060 & 0.007208 & 1.010847 & 0.000072 & 293.7 & 293.6 & 294.6 & 293.6 & 0.76 & 237026. & 0.2146 & 0.2313 \\
\hline 0.006998 & 0.007146 & 1.010847 & 0.000072 & 293.7 & 293.7 & 294.7 & 293.6 & 0.64 & 237070. & 0.2288 & 0.2428 \\
\hline 0.007111 & 0.007260 & 1.010847 & 0.000072 & 293.7 & 293.7 & 294.8 & 293.6 & 0.51 & 237111. & 0.2161 & 0.2415 \\
\hline 0.007065 & 0.007214 & 1.010847 & 0.000072 & 293.8 & 293.7 & 294.7 & 293.6 & 0.38 & 237152. & 0.2154 & 0.2009 \\
\hline 0.007105 & 0.007255 & 1.010847 & 0.000072 & 293.8 & 293.8 & 294.6 & 293.6 & 0.25 & 237198. & 0.2231 & 0.2004 \\
\hline 0.007172 & 0.007323 & 1.010847 & 0.000072 & 293.8 & 293.8 & 294.6 & 293.6 & 0.13 & 237241. & 0.2314 & 0.1928 \\
\hline 0.006849 & 0.007022 & 1.014358 & 0.000078 & 293.4 & 293.4 & 294.6 & 293.7 & 1.59 & 240174. & 0.2232 & 0.2063 \\
\hline 0.006815 & 0.006988 & 1.014358 & 0.000078 & 293.4 & 293.4 & 294.8 & 293.6 & 1.52 & 240224. & 0.2185 & 0.2267 \\
\hline 0.006847 & 0.007020 & 1.014358 & 0.000078 & $\begin{array}{l}293.4 \\
293.4\end{array}$ & 293.4 & 294.6 & 293.6 & 1.40 & 240267. & 0.2184 & 0.2373 \\
\hline 0.006850 & 0.007023 & 1.014358 & 0.000078 & 293.4 & 293.4 & 294.6 & 293.6 & 1.27 & 240313. & 0.2200 & 0.2540 \\
\hline 0.006814 & 0.006986 & $\begin{array}{l}1.0145350 \\
1.014358\end{array}$ & 0.000078 & 293.4 & 293.4 & 294.6 & $\begin{array}{l}293.0 \\
293.6\end{array}$ & 1.14 & 240355. & 0.2192 & 0.2564 \\
\hline 0.006853 & 0.007025 & 1.014358 & 0.000078 & 293.4 & 293.4 & 294.6 & 293.6 & 1.02 & 240396. & 0.2185 & 0.2566 \\
\hline 0.006883 & 0.007056 & 1.014358 & 0.000078 & 293.4 & 293.4 & 294.8 & 293.6 & 0.89 & 240460. & 0.2263 & 0.2379 \\
\hline 0.006854 & 0.007027 & $\begin{array}{l}1.014530 \\
1.014358\end{array}$ & 0.000078 & 293.4 & 293.4 & 294.9 & $\begin{array}{l}293.0 \\
293.6\end{array}$ & 0.76 & 240502. & 0.2264 & 0.2355 \\
\hline 0.006924 & 0.007098 & 1.014358 & 0.000078 & $\begin{array}{l}293.4 \\
293.4\end{array}$ & 293.4 & 294.9 & 293.6 & 0.64 & 240546. & 0.2192 & 0.2505 \\
\hline 0.006944 & 0.007118 & 1.014358 & 0.000078 & 293.4 & 293.4 & 295.1 & 293.6 & 0.51 & 240591. & 0.2255 & 0.2512 \\
\hline 0.007005 & 0.007180 & $\begin{array}{l}1.014500 \\
1.014358\end{array}$ & 0.000078 & 293.4 & 293.4 & $\begin{array}{l}290.1 \\
294.9\end{array}$ & $\begin{array}{l}293.0 \\
293.6\end{array}$ & 0.38 & 240635. & 0.2169 & 0.2541 \\
\hline 0.007047 & 0.007223 & 1.014358 & 0.000078 & $\begin{array}{l}293.4 \\
293.4\end{array}$ & 293.4 & 294.8 & 293.6 & 0.25 & 240679. & 0.2232 & 0.2533 \\
\hline 0.007078 & 0.007254 & 1.014358 & 0.000078 & 293.4 & 293.4 & 294.8 & 293.6 & 0.13 & 240723. & 0.2154 & 0.2519 \\
\hline 0.006790 & 0.006826 & $\begin{array}{l}1.0145000 \\
1.005077\end{array}$ & -0.000003 & 294.1 & 294.0 & 295.3 & $\begin{array}{l}293.0 \\
293.6\end{array}$ & 1.59 & 243116. & 0.2231 & 0.2427 \\
\hline 0.006778 & 0.006814 & 1.005077 & -0.000003 & 294.0 & 294.0 & 295.5 & 293.6 & 1.52 & 243163. & 0.2296 & 0.2392 \\
\hline 0.006690 & 0.006725 & 1.005077 & -0.000003 & 294.0 & 294.0 & 295.7 & 293.6 & 1.40 & 243213. & 0.2255 & 0.2352 \\
\hline 0.006790 & 0.006825 & 1.005077 & -0.000003 & 294.0 & 294.0 & 295.4 & $\begin{array}{l}293.0 \\
293.6\end{array}$ & 1.40 & 243257. & 0.2215 & 0.2321 \\
\hline 0.006798 & 0.006833 & 1.005077 & -0.000003 & 294.0 & 294.0 & $\begin{array}{l}295.4 \\
295.1 \\
\end{array}$ & 293.6 & 1.14 & 243301. & 0.2131 & 0.2323 \\
\hline 0.006825 & 0.006860 & 1.005077 & -0.000003 & 294.0 & 293.9 & $\begin{array}{l}295.1 \\
295.1\end{array}$ & $\begin{array}{l}293.0 \\
293.6\end{array}$ & $\begin{array}{l}1.14 \\
1.02\end{array}$ & 243345. & 0.2231 & 0.2397 \\
\hline 0.006816 & 0.006851 & 1.005077 & -0.000003 & 294.0 & 293.9 & $\begin{array}{l}295.1 \\
295.0\end{array}$ & 293.6 & 0.89 & 243390. & 0.2138 & 0.2493 \\
\hline 0.006828 & 0.006863 & 1.005077 & -0.000003 & 293.9 & 293.9 & 295.4 & 293.6 & 0.76 & 243433. & 0.2296 & 0.2424 \\
\hline $\begin{array}{l}0.0000200 \\
0.006875\end{array}$ & 0.006910 & 1.005077 & -0.000003 & 293.9 & 293.9 & $\begin{array}{l}295.4 \\
295.3\end{array}$ & $\begin{array}{l}293.0 \\
293.6\end{array}$ & 0.64 & 243474. & 0.2263 & 0.2424 \\
\hline 0.006892 & 0.006927 & 1.005077 & -0.000003 & 293.9 & 293.9 & 295.1 & $\begin{array}{l}293.0 \\
293.6\end{array}$ & 0.51 & 243515. & 0.2255 & 0.2316 \\
\hline 0.006982 & 0.007017 & 1.005077 & -0.000003 & 293.9 & 293.9 & 295.0 & 293.6 & 0.38 & 243556. & 0.2223 & 0.2139 \\
\hline $\begin{array}{l}0.006975 \\
0.00975\end{array}$ & 0.007010 & 1.005077 & -0.000003 & $\begin{array}{l}293.5 \\
293.9\end{array}$ & 293.9 & 294.8 & $\begin{array}{l}293.0 \\
293.6\end{array}$ & 0.25 & 243596. & 0.2177 & 0.2067 \\
\hline 0.006999 & 0.007034 & 1.005077 & -0.000003 & 293.9 & 293.9 & 294.8 & 293.6 & 0.13 & 243639. & 0.2239 & 0.2073 \\
\hline 0.006855 & 0.006929 & 1.005073 & 0.000038 & 293.8 & 293.8 & 295.2 & 293.6 & 1.59 & 243799. & 0.2247 & 0.1886 \\
\hline 0.006817 & 0.006890 & 1.005073 & 0.000038 & 293.8 & 293.8 & 295.1 & 293.6 & 1.52 & 243844. & 0.2223 & 0.1999 \\
\hline 0.006834 & 0.006907 & 1.005073 & 0.000038 & 293.8 & 293.8 & 295.0 & 293.6 & 1.40 & 243886. & 0.2247 & 0.2027 \\
\hline 0.006828 & 0.006901 & 1.005073 & 0.000038 & 293.8 & 293.7 & 294.9 & 293.6 & 1.27 & 243929. & 0.2200 & 0.2222 \\
\hline 0.006852 & 0.006925 & 1.005073 & 0.000038 & 293.7 & 293.7 & 294.9 & 293.6 & 1.14 & 243972. & 0.2247 & 0.2475 \\
\hline
\end{tabular}


Table H.1.5 Diesel contamination on oxidized copper surface for $\operatorname{Re}=7000$ and $x_{\mathrm{b}}=0.2 \%$

\begin{tabular}{|c|c|c|c|c|c|c|c|c|c|c|c|}
\hline $\begin{array}{c}F \\
(\mathrm{v})\end{array}$ & $\begin{array}{l}F_{\mathrm{r}} \\
(\mathrm{v})\end{array}$ & $\begin{array}{c}F_{100} \\
(\mathrm{v})\end{array}$ & $\begin{array}{l}F_{0} \\
(\mathrm{v})\end{array}$ & $\begin{array}{l}T_{\mathrm{Ti}} \\
(\mathrm{K})\end{array}$ & $\begin{array}{l}T_{\mathrm{To}} \\
(\mathrm{K})\end{array}$ & $\begin{array}{c}T_{\mathrm{a}} \\
(\mathrm{K})\end{array}$ & $\begin{array}{c}T_{\mathrm{b}} \\
(\mathrm{K})\end{array}$ & $\begin{array}{c}l \\
(\mathrm{~mm})\end{array}$ & $\begin{array}{c}\text { Exposure } \\
\text { time (s) }\end{array}$ & $\begin{array}{c}\text { Turbine } \\
\text { meter } \\
\dot{m}_{\mathrm{w}}(\mathrm{kg} / \mathrm{s})\end{array}$ & $\begin{array}{c}\text { Doppler } \\
\text { meter } \\
\dot{m}_{\mathrm{w}}(\mathrm{kg} / \mathrm{s})\end{array}$ \\
\hline 0.002504 & 0.002483 & 1.005823 & -0.00005 & 297.5 & 297.4 & 296.8 & 293.7 & 1.59 & 4639. & 0.3299 & 0.3423 \\
\hline 0.002400 & 0.002378 & 1.005823 & -0.000050 & 297.5 & 297.5 & 296.6 & 293.7 & 1.52 & 4684. & 0.3265 & 0.3417 \\
\hline 0.02268 & 0.002245 & 1.005823 & -0.000050 & 297.6 & 297.5 & 297.0 & 293.7 & 1.40 & 4728. & 0.3215 & 0.3391 \\
\hline 0.002026 & 0.002000 & 1.005823 & -0.000050 & 297.6 & 297.6 & 297.1 & 293.7 & 1.27 & 4771. & 0.3167 & 0.3415 \\
\hline 0.001853 & 0.001825 & 1.005823 & -0.000050 & 297.6 & 297.6 & 296.9 & 293.7 & 1.14 & 4813. & 0.3300 & 0.3453 \\
\hline 0.001738 & 0.001709 & 1.005823 & -0.000050 & 297.7 & 297.6 & 296.9 & 293.7 & 1.02 & 4857. & 0.3181 & 0.3406 \\
\hline 0.001519 & 0.001487 & 1.005823 & -0.000050 & 297.7 & 297.7 & 296.8 & 293.7 & 0.89 & 4900. & 0.3333 & 0.3420 \\
\hline 0.001320 & 0.001285 & 1.005823 & -0.000050 & 297.7 & 297.7 & 296.8 & 293.7 & 0.76 & 4943. & 0.3214 & 0.3377 \\
\hline 0.001150 & 0.001114 & 1.005823 & -0.000050 & 297.8 & 297.7 & 296.9 & 293.6 & 0.64 & 4987. & 0.3249 & 0.3425 \\
\hline 0.000987 & 0.000949 & 1.005823 & -0.000050 & 297.8 & 297.8 & 297.0 & 293.6 & 0.51 & 5029. & 0.3282 & 0.3401 \\
\hline 0.000803 & 0.000762 & 1.005823 & -0.000050 & 297.8 & 297.8 & 297.1 & 293.7 & 0.38 & 5075. & 0.3214 & 0.3392 \\
\hline 0.000611 & 0.000568 & 1.005823 & -0.000050 & 297.8 & 297.8 & 296.9 & 293.7 & 0.25 & 5122. & 0.3133 & 0.3435 \\
\hline 0.000479 & 0.000434 & 1.005823 & -0.000050 & 297.9 & 297.8 & 297.1 & 293.7 & 0.13 & 5163. & 0.3352 & 0.3391 \\
\hline 0.002624 & 0.002647 & 1.006991 & -0.000013 & 297.9 & 297.9 & 296.8 & 293.7 & 1.59 & 5325. & 0.3232 & 0.3398 \\
\hline 0.002535 & 0.002557 & 1.006991 & -0.000013 & 297.9 & 297.9 & 297.0 & 293.7 & 1.52 & 5366. & 0.3182 & 0.3404 \\
\hline 0.002339 & 0.002358 & 1.006991 & -0.000013 & 298.0 & 297.9 & 296.9 & 293.7 & 1.40 & 5412. & 0.3181 & 0.3514 \\
\hline 0.002156 & 0.002172 & 1.006991 & -0.000013 & 298.0 & 297.9 & 297.0 & 293.7 & 1.27 & 5459. & 0.3197 & 0.3590 \\
\hline 0.001968 & 0.001982 & 1.006991 & -0.000013 & 298.0 & 297.9 & 297.2 & 293.7 & 1.14 & 5511. & 0.3231 & 0.3589 \\
\hline 0.001751 & 0.001762 & 1.006991 & -0.000013 & 298.0 & 297.9 & 297.0 & 293.7 & 1.02 & 5554. & 0.3299 & 0.3511 \\
\hline 0.001554 & 0.001562 & 1.006991 & -0.000013 & 298.0 & 298.0 & 297.0 & 293.7 & 0.89 & 5600. & 0.3317 & 0.3442 \\
\hline 0.001390 & 0.001396 & 1.006991 & -0.000013 & 298.0 & 298.0 & 296.8 & 293.7 & 0.76 & 5642. & 0.3282 & 0.3571 \\
\hline 0.001155 & 0.001158 & 1.006991 & -0.000013 & 298.0 & 298.0 & 296.9 & 293.7 & 0.64 & 5685. & 0.3165 & 0.3454 \\
\hline 0.000955 & 0.000955 & 1.006991 & -0.000013 & 298.0 & 298.0 & 297.0 & 293.7 & 0.51 & 5726. & 0.3317 & 0.3504 \\
\hline 0.000762 & 0.000759 & 1.006991 & -0.000013 & 298.0 & 298.0 & 297.0 & 293.7 & 0.38 & 5771. & 0.3299 & 0.3401 \\
\hline 0.000603 & 0.000598 & 1.006991 & -0.000013 & 298.0 & 298.0 & 296.9 & 293.7 & 0.25 & 5818. & 0.3317 & 0.3394 \\
\hline .000443 & 0.000436 & 1.006991 & -0.000013 & 298.0 & 298.0 & 297.0 & 293.7 & 0.13 & 5865. & 0.3214 & 0.3467 \\
\hline .002757 & 0.002840 & 1.004241 & 0.000055 & 297.4 & 297.3 & 297.3 & 293.7 & 1.59 & 7893. & 0.3316 & 0.3527 \\
\hline .002633 & 0.002714 & 1.004241 & 0.000055 & 297.3 & 297.3 & 297.5 & 293.7 & 1.52 & 7942. & 0.3408 & 0.3492 \\
\hline .002596 & 0.002676 & 1.004241 & 0.000055 & 297.3 & 297.3 & 297.7 & 293.7 & 1.40 & 7988. & 0.3249 & 0.3486 \\
\hline .002495 & 0.002574 & 1.004241 & 0.000055 & 297.3 & 297.3 & 297.4 & 293.7 & 1.27 & 8031. & 0.3335 & 0.3486 \\
\hline .002276 & 0.002354 & 1.004241 & 0.000055 & 297.3 & 297.3 & 297.4 & 293.7 & 1.14 & 8072. & 0.3370 & 0.3499 \\
\hline .002094 & 0.002169 & 1.004241 & 0.000055 & 297.2 & 297.2 & 297.3 & 293.7 & 1.02 & 8115. & 0.3166 & 0.3528 \\
\hline .001767 & 0.001840 & 1.004241 & 0.000055 & 297.2 & 297.2 & 297.6 & 293.7 & 0.89 & 8159. & 0.3282 & 0.3562 \\
\hline .001538 & 0.001608 & 1.004241 & 0.000055 & 297.2 & 297.2 & 297.5 & 293.7 & 0.76 & 8202. & 0.3197 & 0.3500 \\
\hline .001401 & 0.001469 & 1.004241 & 0.000055 & 297.2 & 297.2 & 297.4 & 293.7 & 0.64 & 8245. & 0.3264 & 0.3524 \\
\hline .001210 & 0.001276 & 1.004241 & 0.000055 & 297.2 & 297.1 & 297.5 & 293.7 & 0.51 & 8296. & 0.3335 & 0.3529 \\
\hline .001021 & 0.001086 & 1.004241 & 0.000055 & 297.1 & 297.1 & 297.4 & 293.7 & 0.38 & 8341. & 0.3266 & 0.3500 \\
\hline .000822 & 0.000884 & 1.004241 & 0.000055 & 297.1 & 297.1 & 297.5 & 293.7 & 0.25 & 8392. & 0.3248 & 0.3483 \\
\hline .000718 & 0.000780 & 1.004241 & 0.000055 & 297.1 & 297.1 & 297.6 & 293.7 & 0.13 & 8449. & 0.3335 & 0.3437 \\
\hline .003012 & 0.003122 & 1.010220 & 0.000071 & 295.4 & 295.4 & 297.5 & 293.7 & 1.59 & 11766. & 0.3337 & 0.3416 \\
\hline .002916 & 0.003025 & 1.010220 & 0.000071 & 295.4 & 295.4 & 297.5 & 293.7 & 1.52 & 11806. & 0.3319 & 0.3414 \\
\hline .002828 & 0.002936 & 1.010220 & 0.000071 & 295.4 & 295.4 & 297.6 & 293.7 & 1.40 & 11848. & 0.3372 & 0.3358 \\
\hline .002715 & 0.002821 & 1.010220 & 0.000071 & 295.4 & 295.4 & 297.4 & 293.7 & 1.27 & 11894. & 0.3372 & 0.3367 \\
\hline .002608 & 0.002713 & 1.010220 & 0.000071 & 295.3 & 295.3 & 297.5 & 293.7 & 1.14 & 11937. & 0.3184 & 0.3550 \\
\hline .002429 & 0.002531 & 1.010220 & 0.000071 & 295.3 & 295.3 & 297.6 & 293.7 & 1.02 & 11984. & 0.3266 & 0.3483 \\
\hline .002213 & 0.002313 & 1.010220 & 0.000071 & 295.3 & 295.3 & 297.6 & 293.7 & 0.89 & 12032. & 0.3250 & 0.3441 \\
\hline .002003 & 0.002100 & 1.010220 & 0.000071 & 295.3 & 295.3 & 297.5 & 293.7 & 0.76 & 12082. & 0.3354 & 0.3192 \\
\hline .001850 & 0.001945 & 1.010220 & 0.000071 & 295.3 & 295.3 & 297.4 & 293.7 & 0.64 & 12124. & 0.3374 & 0.3216 \\
\hline .001622 & 0.001714 & 1.010220 & 0.000071 & 295.2 & 295.2 & 297.5 & 293.7 & 0.51 & 12168. & 0.3337 & 0.3134 \\
\hline .001473 & 0.001564 & 1.010220 & 0.000071 & 295.2 & 295.2 & 297.7 & 293.7 & 0.38 & 12208. & 0.3216 & 0.3164 \\
\hline .001245 & 0.001333 & 1.010220 & 0.000071 & 295.2 & 295.2 & 297.4 & 293.7 & 0.25 & 12252. & 0.3284 & 0.3298 \\
\hline .001071 & 0.001156 & 1.010220 & 0.000071 & 295.2 & 295.2 & 297.6 & 293.7 & 0.13 & 12298. & 0.3284 & 0.3386 \\
\hline .003126 & 0.003182 & 1.005162 & 0.000033 & 295.1 & 295.1 & 297.4 & 293.7 & 1.59 & 12461. & 0.3266 & 0.3588 \\
\hline .003037 & 0.003092 & 1.005162 & 0.000033 & 295.1 & 295.1 & 297.4 & 293.7 & 1.52 & 12506. & 0.3216 & 0.3555 \\
\hline .002849 & 0.002903 & 1.005162 & 0.000033 & 295.1 & 295.1 & 297.5 & 293.7 & 1.40 & 12550. & 0.3354 & 0.3592 \\
\hline .002710 & 0.002763 & 1.005162 & 0.000033 & 295.0 & 295.0 & 297.3 & 293.7 & 1.27 & 12595. & 0.3185 & 0.3491 \\
\hline .002642 & 0.002694 & 1.005162 & 0.000033 & 295.0 & 295.0 & 297.6 & 293.7 & 1.14 & 12637. & 0.3267 & 0.3426 \\
\hline
\end{tabular}


Table H.1.6 Tap water flushing after $\mathrm{Re}=4600$ contamination tests at $x_{\mathrm{b}}=0.2 \%$

\begin{tabular}{|c|c|c|c|c|c|c|c|c|c|c|c|}
\hline \multicolumn{12}{|c|}{ Tap water flushing after $\mathrm{Re}=4600$ contamination tests at $x_{\mathrm{b}}=0.2 \%$ (file:flsh45c1.tbl) } \\
\hline $\begin{array}{c}F \\
(\mathrm{v})\end{array}$ & $\begin{array}{c}F_{\mathrm{r}} \\
(\mathrm{v})\end{array}$ & $\begin{array}{c}F_{100} \\
(\mathrm{v})\end{array}$ & $\begin{array}{l}F_{0} \\
(\mathrm{v})\end{array}$ & $\begin{array}{l}T_{\mathrm{Ti}} \\
(\mathrm{K})\end{array}$ & $\begin{array}{l}T_{\mathrm{To}} \\
(\mathrm{K})\end{array}$ & $\begin{array}{c}T_{\mathrm{a}} \\
(\mathrm{K})\end{array}$ & $\begin{array}{c}T_{\mathrm{b}} \\
(\mathrm{K})\end{array}$ & $\begin{array}{c}l \\
(\mathrm{~mm})\end{array}$ & $\begin{array}{l}\text { Exposure } \\
\text { time (s) }\end{array}$ & $\begin{array}{l}\text { Turbine } \\
\text { meter } \\
\dot{m}_{\mathrm{w}}(\mathrm{kg} / \mathrm{s})\end{array}$ & $\begin{array}{l}\text { Doppler } \\
\text { meter }\end{array}$ \\
\hline 0.004939 & 0.004940 & 0.996651 & -.000033 & 300.0 & 300.1 & 295.2 & 293.6 & 1.59 & 2413. & N/A & N/A \\
\hline 0.004815 & 0.004814 & 0.996651 & -.000033 & 299.9 & 300.0 & 295.5 & 293.6 & 1.52 & 2467. & N/A & N/A \\
\hline 0.004740 & 0.004738 & 0.996651 & -.000033 & 299.9 & 299.9 & 295.3 & 293.6 & 1.40 & 2522. & N/A & N/A \\
\hline 0.004705 & 0.004703 & 0.996651 & -.000033 & 299.9 & 299.9 & 295.6 & 293.6 & 1.27 & 2574. & N/A & N/A \\
\hline 0.004696 & 0.004693 & 0.996651 & -.000033 & 299.8 & 299.8 & 295.6 & 293.6 & 1.14 & 2634. & N/A & N/A \\
\hline 0.004677 & 0.004674 & 0.996651 & -.000033 & 299.8 & 299.8 & 295.5 & 293.6 & 1.02 & 2686. & N/A & N/A \\
\hline 0.004686 & 0.004683 & 0.996651 & -.000033 & 299.7 & 299.7 & 295.4 & 293.6 & 0.89 & 2744. & N/A & N/A \\
\hline 0.004756 & 0.004753 & 0.996651 & -.000033 & 299.7 & 299.6 & 295.6 & 293.6 & 0.76 & 2796. & N/A & N/A \\
\hline 0.004820 & 0.004817 & 0.996651 & -.000033 & 299.6 & 299.6 & 295.3 & 293.5 & 0.64 & 2849. & N/A & N/A \\
\hline 0.004874 & 0.004870 & 0.996651 & -.000033 & 299.5 & 299.5 & 295.2 & 293.6 & 0.51 & 2898. & N/A & N/A \\
\hline 0.004914 & 0.004909 & 0.996651 & -.000033 & 299.4 & 299.4 & 295.3 & 293.6 & 0.38 & 2953. & N/A & N/A \\
\hline 0.004973 & 0.004968 & 0.996651 & -.000033 & 299.4 & 299.3 & 295.4 & 293.6 & 0.25 & 3000. & N/A & N/A \\
\hline 0.004295 & 0.004363 & 0.994784 & 0.000053 & 299.1 & 299.1 & 295.7 & 293.6 & 1.59 & 3214. & N/A & N/A \\
\hline 0.004260 & 0.004327 & 0.994784 & 0.000053 & 299.0 & 299.0 & 295.7 & 293.6 & 1.40 & 3271. & N/A & N/A \\
\hline 0.004307 & 0.004374 & 0.994784 & 0.000053 & 299.0 & 298.9 & 295.7 & 293.6 & 1.14 & 3326. & N/A & N/A \\
\hline 0.004450 & 0.004518 & 0.994784 & 0.000053 & 298.9 & 298.9 & 295.8 & 293.6 & 0.89 & 3380. & N/A & N/A \\
\hline 0.004456 & 0.004524 & 0.994784 & 0.000053 & 298.9 & 298.8 & 295.8 & 293.6 & 0.76 & 3430. & N/A & N/A \\
\hline 0.004555 & 0.004622 & 0.994784 & 0.000053 & 298.8 & 298.8 & 295.7 & 293.6 & 0.64 & 3481. & N/A & N/A \\
\hline 0.004656 & 0.004723 & 0.994784 & 0.000053 & 298.8 & 298.7 & 295.7 & 293.6 & 0.51 & 3530. & N/A & N/A \\
\hline 0.004733 & 0.004799 & 0.994784 & 0.000053 & 298.7 & 298.7 & 295.8 & 293.6 & 0.38 & 3581. & N/A & N/A \\
\hline 0.004788 & 0.004854 & 0.994784 & 0.000053 & 298.7 & 298.6 & 296.0 & 293.6 & 0.25 & 3631. & N/A & N/A \\
\hline 0.004939 & 0.005006 & 0.994784 & 0.000053 & 298.6 & 298.6 & 296.0 & 293.6 & 0.13 & 3684. & N/A & N/A \\
\hline 0.003415 & 0.003450 & 1.009424 & 0.000007 & 292.8 & 292.7 & 295.3 & 293.6 & 1.59 & 25131. & N/A & N/A \\
\hline 0.003385 & 0.003419 & 1.009424 & 0.000007 & 292.8 & 292.7 & 295.4 & 293.6 & 1.40 & 25189. & N/A & N/A \\
\hline 0.003506 & 0.003542 & 1.009424 & 0.000007 & 292.8 & 292.7 & 295.3 & 293.6 & 1.14 & 25244. & N/A & N/A \\
\hline 0.003575 & 0.003611 & 1.009424 & 0.000007 & 292.8 & 292.7 & 295.3 & 293.6 & 0.89 & 25297. & N/A & N/A \\
\hline 0.003776 & 0.003814 & 1.009424 & 0.000007 & 292.8 & 292.7 & 295.4 & 293.6 & 0.64 & 25351. & N/A & N/A \\
\hline 0.003805 & 0.003843 & 1.009424 & 0.000007 & 292.8 & 292.7 & 295.3 & 293.6 & 0.51 & 25403. & N/A & N/A \\
\hline 0.003859 & 0.003897 & 1.009424 & 0.000007 & 292.8 & 292.7 & 295.4 & 293.6 & 0.38 & 25455. & N/A & N/A \\
\hline 0.004004 & 0.004044 & 1.009424 & 0.000007 & 292.8 & 292.7 & 295.5 & 293.6 & 0.25 & 25506. & N/A & N/A \\
\hline 0.004149 & 0.004189 & 1.009424 & 0.000007 & 292.8 & 292.7 & 295.5 & 293.6 & 0.13 & 25557. & N/A & N/A \\
\hline 0.003426 & 0.003475 & 1.007668 & 0.000027 & 292.9 & 292.8 & 295.5 & 293.6 & 1.59 & 25789. & N/A & N/A \\
\hline 0.003328 & 0.003377 & 1.007668 & 0.000027 & 292.9 & 292.8 & 295.4 & 293.6 & 1.40 & 25844. & N/A & N/A \\
\hline 0.003477 & 0.003527 & 1.007668 & 0.000027 & 292.9 & 292.8 & 295.6 & 293.6 & 1.14 & 25894. & N/A & N/A \\
\hline 0.003526 & 0.003576 & 1.007668 & 0.000027 & 292.9 & 292.8 & 295.5 & 293.6 & 0.89 & 25951. & N/A & N/A \\
\hline 0.003649 & 0.003700 & 1.007668 & 0.000027 & 292.9 & 292.8 & 295.3 & 293.6 & 0.64 & 26010. & N/A & N/A \\
\hline 0.003825 & 0.003878 & 1.007668 & 0.000027 & 292.9 & 292.9 & 295.5 & 293.6 & 0.38 & 26058. & N/A & N/A \\
\hline 0.004136 & 0.004190 & 1.007668 & 0.000027 & 292.9 & 292.9 & 295.4 & 293.6 & 0.13 & 26106. & N/A & N/A \\
\hline 0.002337 & 0.003209 & 1.001896 & 0.000868 & 293.9 & 293.9 & 295.2 & 293.6 & 1.59 & 83821. & N/A & N/A \\
\hline 0.002272 & 0.002414 & 1.002567 & 0.000135 & 294.0 & 293.9 & 295.4 & 293.6 & 1.52 & 84083. & N/A & N/A \\
\hline 0.002263 & 0.002405 & 1.002567 & 0.000135 & 293.9 & 293.9 & 295.4 & 293.6 & 1.40 & 84145. & N/A & N/A \\
\hline 0.002284 & 0.002426 & 1.002567 & 0.000135 & 294.0 & 293.9 & 295.6 & 293.6 & 1.27 & 84204. & N/A & N/A \\
\hline 0.002328 & 0.002470 & 1.002567 & 0.000135 & 294.0 & 293.9 & 295.5 & 293.6 & 1.14 & 84256. & N/A & N/A \\
\hline 0.002313 & 0.002455 & 1.002567 & 0.000135 & 294.0 & 293.9 & 295.4 & 293.6 & 1.02 & 84311. & N/A & N/A \\
\hline 0.002436 & 0.002578 & 1.002567 & 0.000135 & 293.9 & 293.9 & 295.5 & 293.6 & 0.89 & 84369. & N/A & N/A \\
\hline 0.002417 & 0.002559 & 1.002567 & 0.000135 & 293.9 & 293.9 & 295.5 & 293.6 & 0.76 & 84456. & N/A & N/A \\
\hline 0.002492 & 0.002635 & 1.002567 & 0.000135 & 293.9 & 293.9 & 295.6 & 293.6 & 0.64 & 84513. & N/A & N/A \\
\hline 0.002559 & 0.002702 & 1.002567 & 0.000135 & 293.9 & 293.9 & 295.4 & 293.6 & 0.51 & 84590. & N/A & N/A \\
\hline 0.002583 & 0.002725 & 1.002567 & 0.000135 & 294.0 & 293.9 & 295.5 & 293.6 & 0.38 & 84641. & N/A & N/A \\
\hline 0.002656 & 0.002799 & 1.002567 & 0.000135 & 293.9 & 293.9 & 295.6 & 293.6 & 0.25 & 84697. & N/A & N/A \\
\hline 0.002766 & 0.002909 & 1.002567 & 0.000135 & 294.0 & 293.9 & 295.5 & 293.6 & 0.13 & 84755. & N/A & N/A \\
\hline 0.002256 & 0.002399 & 1.002415 & 0.000137 & 294.1 & 294.0 & 295.6 & 293.6 & 1.59 & 85420. & N/A & N/A \\
\hline 0.002223 & 0.002366 & 1.002415 & 0.000137 & 294.1 & 294.0 & 295.4 & 293.6 & 1.52 & 85482. & N/A & N/A \\
\hline 0.002229 & 0.002373 & 1.002415 & 0.000137 & 294.1 & 294.0 & 295.4 & 293.6 & 1.40 & 85542. & N/A & N/A \\
\hline 0.002258 & 0.002402 & 1.002415 & 0.000137 & 294.1 & 294.0 & 295.4 & 293.6 & 1.27 & 85595. & N/A & N/A \\
\hline 0.002341 & 0.002485 & 1.002415 & 0.000137 & 294.1 & 294.0 & 295.5 & 293.6 & 1.14 & 85648. & N/A & N/A \\
\hline 0.002334 & 0.002478 & 1.002415 & 0.000137 & 294.1 & 294.0 & 295.5 & 293.6 & 1.02 & 85697. & N/A & N/A \\
\hline
\end{tabular}


Table H.1.7 Diesel contamination on oxidized copper surface for $\mathrm{Re}=0$ and $x_{\mathrm{b}}=0.3 \%$

\begin{tabular}{|c|c|c|c|c|c|c|c|c|c|c|c|}
\hline \multicolumn{12}{|c|}{ Diesel contamination on oxidized copper surface for $\mathrm{Re}=0$ and $x_{\mathrm{b}}=0.3 \%$ (file:trv0con2.tbl) } \\
\hline $\begin{array}{c}F \\
(\mathrm{v})\end{array}$ & $\begin{array}{c}F_{\mathrm{r}} \\
(\mathrm{v})\end{array}$ & $\begin{array}{c}F_{100} \\
(\mathrm{v})\end{array}$ & $\begin{array}{l}F_{0} \\
(\mathrm{v})\end{array}$ & $\begin{array}{l}T_{\mathrm{Ti}} \\
(\mathrm{K})\end{array}$ & $\begin{array}{l}T_{\mathrm{To}} \\
(\mathrm{K})\end{array}$ & $\begin{array}{c}T_{\mathrm{a}} \\
(\mathrm{K})\end{array}$ & $\begin{array}{c}T_{\mathrm{b}} \\
(\mathrm{K})\end{array}$ & $\begin{array}{c}l \\
(\mathrm{~mm})\end{array}$ & $\begin{array}{l}\text { Exposure } \\
\text { time (s) }\end{array}$ & $\begin{array}{c}\text { Turbine } \\
\text { meter } \\
\dot{m}_{\mathrm{w}}(\mathrm{kg} / \mathrm{s})\end{array}$ & $\begin{array}{c}\text { Doppler } \\
\text { meter } \\
\dot{m}_{\mathrm{w}}(\mathrm{kg} / \mathrm{s})\end{array}$ \\
\hline 0.004440 & 0.004456 & 1.007538 & -0.000029 & 295.4 & 295.4 & 294.5 & 293.7 & 1.59 & 2476. & 0.0000 & 0.0626 \\
\hline 0.004311 & 0.004326 & 1.007538 & -0.000029 & 295.4 & 295.4 & 294.6 & 293.7 & 1.52 & 2532. & 0.0000 & 0.0627 \\
\hline 0.004040 & 0.004052 & 1.007538 & -0.000029 & 295.4 & 295.3 & 294.5 & 293.7 & 1.40 & 2592. & 0.0000 & 0.0626 \\
\hline 0.003770 & 0.003779 & 1.007538 & -0.000029 & 295.4 & 295.3 & 294.4 & 293.7 & 1.27 & 2647. & 0.0000 & 0.0626 \\
\hline 0.003469 & 0.003475 & 1.007538 & -0.000029 & 295.4 & 295.3 & 294.5 & 293.7 & 1.14 & 2702. & 0.0000 & 0.0626 \\
\hline 0.003200 & 0.003204 & 1.007538 & -0.000029 & 295.3 & 295.3 & 294.5 & 293.7 & 1.02 & 2763. & 0.0000 & 0.0627 \\
\hline 0.002949 & 0.002949 & 1.007538 & -0.000029 & 295.3 & 295.3 & 294.4 & 293.7 & 0.89 & 2823. & 0.0000 & 0.0626 \\
\hline 0.002672 & 0.002670 & 1.007538 & -0.000029 & 295.3 & 295.3 & 294.4 & 293.7 & 0.76 & 2880. & 0.0000 & 0.0626 \\
\hline 0.002325 & 0.002319 & 1.007538 & -0.000029 & 295.3 & 295.3 & 294.4 & 293.7 & 0.64 & 2939. & 0.0000 & 0.0626 \\
\hline 0.002060 & 0.002052 & 1.007538 & -0.000029 & 295.3 & 295.2 & 294.4 & 293.7 & 0.51 & 3000. & 0.0000 & 0.0626 \\
\hline 0.001739 & 0.001727 & 1.007538 & -0.000029 & 295.3 & 295.2 & 294.4 & 293.7 & 0.38 & 3056. & 0.0000 & 0.0626 \\
\hline 0.001418 & 0.001404 & 1.007538 & -0.000029 & 295.3 & 295.2 & 294.5 & 293.7 & 0.25 & 3118. & 0.0000 & 0.0627 \\
\hline 0.001074 & 0.001055 & 1.007538 & -0.000029 & 295.3 & 295.2 & 294.6 & 293.7 & 0.13 & 3178. & 0.0000 & 0.0626 \\
\hline 0.004750 & 0.004793 & 1.009045 & -0.000006 & 294.4 & 294.8 & 294.1 & 293.7 & 1.59 & 406841. & 0.0000 & 0.0713 \\
\hline 0.004606 & 0.004648 & 1.009045 & -0.000006 & 294.4 & 294.7 & 294.1 & 293.7 & 1.52 & 406899. & 0.0000 & 0.0792 \\
\hline 0.004393 & 0.004432 & 1.009045 & -0.000006 & 294.4 & 294.8 & 294.1 & 293.7 & 1.40 & 406954. & 0.0000 & 0.0741 \\
\hline 0.004173 & 0.004211 & 1.009045 & -0.000006 & 294.4 & 294.8 & 294.1 & 293.7 & 1.27 & 407010. & 0.0000 & 0.0734 \\
\hline 0.003978 & 0.004013 & 1.009045 & -0.000006 & 294.4 & 294.7 & 294.1 & 293.7 & 1.14 & 407065. & 0.0000 & 0.0807 \\
\hline 0.003783 & 0.003816 & 1.009045 & -0.000006 & 294.4 & 294.8 & 294.1 & 293.7 & 1.02 & 407122. & 0.0000 & 0.0793 \\
\hline 0.003533 & 0.003564 & 1.009045 & -0.000006 & 294.4 & 294.8 & 294.2 & 293.7 & 0.89 & 407178. & 0.0000 & 0.0808 \\
\hline 0.003261 & 0.003289 & 1.009045 & -0.000006 & 294.4 & 294.8 & 294.1 & 293.7 & 0.76 & 407234. & 0.0000 & 0.0738 \\
\hline 0.003042 & 0.003067 & 1.009045 & -0.000006 & 294.4 & 294.8 & 294.1 & 293.7 & 0.64 & 407289. & 0.0000 & 0.0788 \\
\hline 0.002831 & 0.002854 & 1.009045 & -0.000006 & 294.4 & 294.8 & 294.1 & 293.7 & 0.51 & 407354. & 0.0000 & 0.0823 \\
\hline 0.002561 & 0.002581 & 1.009045 & -0.000006 & 294.4 & 294.8 & 294.1 & 293.7 & 0.38 & 407410. & 0.0000 & 0.0779 \\
\hline 0.002334 & 0.002352 & 1.009045 & -0.000006 & 294.4 & 294.8 & 294.1 & 293.7 & 0.25 & 407466. & 0.0000 & 0.0794 \\
\hline 0.001987 & 0.002001 & 1.009045 & -0.000006 & 294.4 & 294.8 & 294.1 & 293.7 & 0.13 & 407521. & 0.0000 & 0.0817 \\
\hline 0.005085 & 0.005157 & 1.000987 & 0.000058 & 294.5 & 294.9 & 294.3 & 293.7 & 1.59 & 410253. & 0.0000 & 0.0793 \\
\hline 0.005006 & 0.005077 & 1.000987 & 0.000058 & 294.5 & 294.9 & 294.2 & 293.7 & 1.52 & 410306. & 0.0000 & 0.0785 \\
\hline 0.004853 & 0.004923 & 1.000987 & 0.000058 & 294.5 & 294.9 & 294.3 & 293.7 & 1.40 & 410359. & 0.0000 & 0.0755 \\
\hline 0.004663 & 0.004733 & 1.000987 & 0.000058 & 294.5 & 294.9 & 294.2 & 293.7 & 1.27 & 410412. & 0.0000 & 0.0803 \\
\hline 0.004474 & 0.004544 & 1.000987 & 0.000058 & 294.5 & 294.9 & 294.2 & 293.7 & 1.14 & 410468. & 0.0000 & 0.0799 \\
\hline 0.004302 & 0.004371 & 1.000987 & 0.000058 & 294.5 & 294.9 & 294.3 & 293.7 & 1.02 & 410522. & 0.0000 & 0.0747 \\
\hline 0.004080 & 0.004149 & 1.000987 & 0.000058 & 294.5 & 294.9 & 294.2 & 293.7 & 0.89 & 410577. & 0.0000 & 0.0721 \\
\hline 0.003813 & 0.003881 & 1.000987 & 0.000058 & 294.5 & 294.9 & 294.2 & 293.7 & 0.76 & 410632. & 0.0000 & 0.0768 \\
\hline 0.003700 & 0.003768 & 1.000987 & 0.000058 & 294.5 & 294.9 & 294.3 & 293.7 & 0.64 & 410685. & 0.0000 & 0.0627 \\
\hline 0.003460 & 0.003527 & 1.000987 & 0.000058 & 294.5 & 294.9 & 294.3 & 293.7 & 0.51 & 410739. & 0.0000 & 0.0627 \\
\hline 0.003296 & 0.003363 & 1.000987 & 0.000058 & 294.5 & 294.9 & 294.3 & 293.7 & 0.38 & 410798. & 0.0000 & 0.0627 \\
\hline 0.003000 & 0.003066 & 1.000987 & 0.000058 & 294.5 & 294.9 & 294.3 & 293.7 & 0.25 & 410853. & 0.0000 & 0.0627 \\
\hline 0.002693 & 0.002758 & 1.000987 & 0.000058 & 294.5 & 294.9 & 294.3 & 293.7 & 0.13 & 410915. & 0.0000 & 0.0627 \\
\hline 0.005401 & 0.005435 & 1.003869 & 0.000004 & 294.6 & 295.1 & 294.2 & 293.7 & 1.59 & 414268. & 0.0000 & 0.0740 \\
\hline 0.005254 & 0.005287 & 1.003869 & 0.000004 & 294.6 & 295.1 & 294.2 & 293.7 & 1.52 & 414324. & 0.0000 & 0.0789 \\
\hline 0.005067 & 0.005099 & 1.003869 & 0.000004 & 294.6 & 295.1 & 294.2 & 293.7 & 1.40 & 414380. & 0.0000 & 0.0780 \\
\hline 0.004929 & 0.004961 & 1.003869 & 0.000004 & 294.6 & 295.1 & 294.2 & 293.7 & 1.27 & 414435. & 0.0000 & 0.0705 \\
\hline 0.004675 & 0.004706 & 1.003869 & 0.000004 & 294.6 & 295.1 & 294.2 & 293.7 & 1.14 & 414494. & 0.0000 & 0.0691 \\
\hline 0.004599 & 0.004629 & 1.003869 & 0.000004 & 294.6 & 295.1 & 294.2 & 293.7 & 1.02 & 414545. & 0.0000 & 0.0627 \\
\hline 0.004409 & 0.004438 & 1.003869 & 0.000004 & 294.6 & 295.1 & 294.2 & 293.7 & 0.89 & 414605. & 0.0000 & 0.0724 \\
\hline 0.004208 & 0.004236 & 1.003869 & 0.000004 & 294.6 & 295.1 & 294.2 & 293.7 & 0.76 & 414662. & 0.0000 & 0.0627 \\
\hline 0.004000 & 0.004026 & 1.003869 & 0.000004 & 294.6 & 295.1 & 294.2 & 293.7 & 0.64 & 414717. & 0.0000 & 0.0627 \\
\hline 0.003820 & 0.003845 & 1.003869 & 0.000004 & 294.6 & 295.1 & 294.2 & 293.7 & 0.51 & 414775. & 0.0000 & 0.0691 \\
\hline 0.003647 & 0.003672 & 1.003869 & 0.000004 & 294.6 & 295.1 & 294.2 & 293.7 & 0.38 & 414834. & 0.0000 & 0.0727 \\
\hline 0.003382 & 0.003405 & 1.003869 & 0.000004 & 294.6 & 295.1 & 294.2 & 293.7 & 0.25 & 414889. & 0.0000 & 0.0698 \\
\hline 0.003199 & 0.003221 & 1.003869 & 0.000004 & 294.6 & 295.1 & 294.2 & 293.7 & 0.13 & 414947. & 0.0000 & 0.0627 \\
\hline 0.005584 & 0.005656 & 1.003422 & 0.000044 & 294.6 & 295.1 & 294.3 & 293.7 & 1.59 & 418388. & 0.0000 & 0.0627 \\
\hline 0.005722 & 0.005796 & 1.003422 & 0.000044 & 294.6 & 295.1 & 294.3 & 293.7 & 1.52 & 418457. & 0.0000 & 0.0627 \\
\hline 0.005533 & 0.005605 & 1.003422 & 0.000044 & 294.6 & 295.1 & 294.3 & 293.7 & 1.40 & 418510. & 0.0000 & 0.0627 \\
\hline 0.005359 & 0.005431 & 1.003422 & 0.000044 & 294.6 & 295.1 & 294.3 & 293.7 & 1.27 & 418565. & 0.0000 & 0.0627 \\
\hline 0.005183 & 0.005254 & 1.003422 & 0.000044 & 294.6 & 295.1 & 294.2 & 293.7 & 1.14 & 418620. & 0.0000 & 0.0627 \\
\hline 0.005061 & 0.005131 & 1.003422 & 0.000044 & 294.6 & 295.1 & 294.2 & 293.7 & 1.02 & 418674. & 0.0000 & 0.0627 \\
\hline
\end{tabular}


Table H.1.8 Diesel contamination on oxidized copper surface for $\mathrm{Re}=2000$ and $x_{\mathrm{b}}=0.3 \%$

\begin{tabular}{|c|c|c|c|c|c|c|c|c|c|c|c|}
\hline \multicolumn{12}{|c|}{$\begin{array}{c}\text { Diesel contamination on oxidized copper surface for } \mathrm{Re}=2000 \text { and } x_{\mathrm{b}}=0.3 \% \\
\text { (file:trv15con2.tbl) }\end{array}$} \\
\hline $\begin{array}{l}F \\
(\mathrm{v})\end{array}$ & $\begin{array}{l}F_{\mathrm{r}} \\
(\mathrm{v})\end{array}$ & $\begin{array}{c}F_{100} \\
(\mathrm{~V})\end{array}$ & $\begin{array}{l}F_{0} \\
(\mathrm{v})\end{array}$ & $\begin{array}{l}T_{\mathrm{Ti}} \\
(\mathrm{K})\end{array}$ & $\begin{array}{l}T_{\mathrm{To}} \\
(\mathrm{K})\end{array}$ & $\begin{array}{l}T_{\mathrm{a}} \\
(\mathrm{K})\end{array}$ & $\begin{array}{l}T_{\mathrm{b}} \\
(\mathrm{K})\end{array}$ & $\begin{array}{c}l \\
(\mathrm{~mm})\end{array}$ & $\begin{array}{c}\text { Exposure } \\
\text { time (s) }\end{array}$ & $\begin{array}{c}\text { Turbine } \\
\text { meter } \\
\dot{m}_{\mathrm{w}}(\mathrm{kg} / \mathrm{s})\end{array}$ & 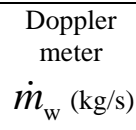 \\
\hline 0.0035970 & 0.0036550 & 1.005509 & 0.0000330 & 294.6 & 294.4 & 294.3 & 293.7 & 1.59 & 613. & 0.0906 & 0.0623 \\
\hline 0.003322 & 0.003378 & 1.005509 & 0.000033 & 294.6 & 294.4 & 294.7 & 293.7 & 1.52 & 749. & 0.0931 & 0.0623 \\
\hline 0.003042 & 0.003096 & 1.005509 & 0.000033 & 294.6 & 294.4 & 294.4 & 293.7 & 1.40 & 792. & 0.0942 & 0.0623 \\
\hline 0.002723 & 0.002775 & 1.005509 & 0.000033 & 294.6 & 294.4 & 294.8 & 293.7 & 1.27 & 837. & 0.0904 & 0.0623 \\
\hline 0.002429 & 0.002479 & 1.005509 & 0.000033 & 294.6 & 294.4 & 294.6 & 293.7 & 1.14 & 881. & 0.0909 & 0.0623 \\
\hline 0.002193 & 0.002241 & 1.005509 & 0.000033 & 294.5 & 294.4 & 294.7 & 293.7 & 1.02 & 922. & 0.0942 & 0.0623 \\
\hline 0.001812 & 0.001857 & 1.005509 & 0.000033 & 294.5 & 294.4 & 294.7 & 293.6 & 0.89 & 966. & 0.0952 & 0.0623 \\
\hline 0.001578 & 0.001622 & 1.005509 & 0.000033 & 294.5 & 294.4 & 294.6 & 293.7 & 0.76 & 1008. & 0.0935 & 0.0623 \\
\hline 0.001261 & 0.001303 & 1.005509 & 0.000033 & 294.5 & 294.4 & 294.4 & 293.7 & 0.64 & 1054. & 0.0920 & 0.0623 \\
\hline 0.000910 & 0.000949 & 1.005509 & 0.000033 & 294.5 & 294.4 & 294.4 & 293.7 & 0.51 & 1097. & 0.0943 & 0.0623 \\
\hline 0.000618 & 0.000656 & 1.005509 & 0.000033 & 294.5 & 294.4 & 294.3 & 293.7 & 0.38 & 1138. & 0.0887 & 0.0623 \\
\hline 0.000226 & 0.000261 & 1.005509 & 0.000033 & 294.4 & 294.4 & 294.7 & 293.7 & 0.25 & 1181. & 0.0952 & .0623 \\
\hline-0.000086 & -0.000054 & 1.005509 & 0.000033 & 294.4 & 294.4 & 294.2 & 293.7 & 0.13 & 1226. & 0.0926 & .0623 \\
\hline 0.003653 & 0.003637 & 1.003232 & -0.000032 & 294.4 & 294.3 & 294.5 & 293.7 & 1.59 & 1374. & 0.0933 & .0623 \\
\hline 0.003463 & 0.003446 & 1.003232 & -0.000032 & 294.4 & 294.3 & 294.3 & 293.7 & 1.52 & 1422. & 0.0900 & 0.0623 \\
\hline 0.003191 & 0.003172 & 1.003232 & -0.000032 & 294.3 & 294.3 & 294.5 & 293.7 & 1.40 & 1468. & 0.0942 & 0.0623 \\
\hline 0.002991 & 0.002972 & 1.003232 & -0.0000 & 294.3 & 294.3 & 294.0 & 293.7 & 1.27 & 1513. & 0.0945 & 0.0623 \\
\hline 0.002751 & 0.002730 & 1.003232 & -0.000032 & 294.3 & 294.2 & 294.7 & 293.7 & 1.14 & 1557. & 0.0896 & .0623 \\
\hline 0.002472 & 0.002451 & 1.003232 & -0.000032 & 294.3 & 294.2 & 294.6 & 293.6 & 1.02 & 1606. & 0.0906 & 0.0623 \\
\hline 0.002169 & 0.002146 & 1.003232 & -0.000032 & 294.2 & 294.2 & 294.4 & 293.7 & 0.89 & 1652. & 0.0939 & 0.0623 \\
\hline 0.001908 & 0.001884 & 1.003232 & -0.000032 & 294.2 & 294.2 & 294.4 & 293.7 & 0.76 & 1694. & 0.0934 & 0.0623 \\
\hline 0.001576 & 0.001550 & 1.003232 & -0.000032 & 294.2 & 294.1 & 294.4 & 293.7 & 0.64 & 1739. & 0.0914 & 0.0623 \\
\hline 0.001306 & 0.001279 & 1.003232 & -0.000032 & 294.2 & 294.1 & 294.2 & 293.7 & 0.51 & 1786. & 0.0916 & 0.0623 \\
\hline 0.001026 & 0.000998 & 1.003232 & -0.000032 & 294.1 & 294.1 & 294.2 & 293.6 & 0.38 & 1831. & 0.0879 & 0.0623 \\
\hline 0.000778 & 0.000749 & 1.003232 & -0.000032 & 294.1 & 294.1 & 294.4 & 293.7 & 0.25 & 1875. & 0.0936 & .0623 \\
\hline 0.000533 & 0.000503 & 1.003232 & -0.000032 & 294.1 & 294.1 & 294.5 & 293.7 & 0.13 & 1919. & 0.0892 & .0623 \\
\hline 0.012290 & 0.012345 & 1.004488 & 0.000009 & 293.3 & 293.2 & 294.2 & 293.7 & 1.59 & 5348. & 0.0890 & .0623 \\
\hline 0.012646 & 0.012703 & 1.004488 & 0.000009 & 293.3 & 293.2 & 294.2 & 293.7 & 1.52 & 5391. & .0890 & 0.0623 \\
\hline 0.012457 & 0.012514 & 1.004488 & 0.000009 & 293.3 & 293.2 & 294.1 & 293.7 & 1.40 & 5440. & 0.0909 & 0.0623 \\
\hline 0.012575 & 0.012632 & 1.004488 & 0.0 & 293.3 & 293.3 & 294.2 & 293.7 & 1.27 & 5485. & 0.0846 & 0.0623 \\
\hline 0.013596 & 0.013657 & 1.004488 & 0.000009 & 293.3 & 293.3 & 294.6 & 293.6 & 1.14 & 5528. & 0.0882 & 0.0623 \\
\hline 0.012922 & 0.012981 & 1.004488 & 0.000009 & 293.3 & 293.3 & 294.1 & 293.7 & 1.02 & 5573. & 0.0835 & 0.0623 \\
\hline 0.011067 & 0.011119 & 1.004488 & 0.000009 & 293.4 & 293.3 & 294.2 & 293.7 & 0.89 & 5620. & 0.0877 & 0.0623 \\
\hline 0.009262 & 0.009308 & 1.004488 & 0.000009 & 293.4 & 293.3 & 294.0 & 293.6 & 0.76 & 5667. & 0.0891 & 0.0623 \\
\hline 0.009017 & 0.009061 & 1.004488 & 0.000009 & 293.4 & 293.3 & 294.6 & 293.7 & 0.64 & 5712. & 0.0857 & 0.0623 \\
\hline 0.009151 & 0.009196 & 1.004488 & 0.000009 & 293.4 & 293.4 & 294.6 & 293.7 & 0.51 & 5756. & 0.0839 & 0.0623 \\
\hline 0.009208 & 0.009254 & 1.004488 & 0.000009 & 293.4 & 293.4 & 294.5 & 293.7 & 0.38 & 5804. & 0.0856 & 0.0623 \\
\hline 0.009529 & 0.009577 & 1.004488 & 0.000009 & 293.4 & 293.4 & 294.3 & 293.7 & 0.25 & 5855. & 0.0873 & 0.0623 \\
\hline 0.010371 & 0.010423 & 1.004488 & 0.000009 & 293.5 & 293.4 & 294.6 & 293.7 & 0.13 & 5910. & 0.0827 & 0.0623 \\
\hline 0.018959 & 0.019067 & 1.005701 & 0.000000 & 293.7 & 293.6 & 294.4 & 293.7 & 1.59 & 11545. & 0.0909 & 0.0623 \\
\hline 0.019063 & 0.019172 & 1.005701 & 0.000000 & 293.7 & 293.7 & 294.2 & 293.7 & 1.52 & 11590. & 0.0906 & 0.0623 \\
\hline 0.019103 & 0.019213 & 1.005701 & 0.000000 & 293.7 & 293.7 & 294.2 & 293.7 & 1.40 & 11635. & 0.0905 & 0.0623 \\
\hline 0.019066 & 0.019176 & 1.005701 & 0.000000 & 293.7 & 293.7 & 294.5 & 293.7 & 1.27 & 11678. & 0.0897 & 0.0623 \\
\hline 0.019079 & 0.019190 & 1.005701 & 0.000000 & 293.8 & 293.7 & 294.6 & 293.7 & 1.14 & 11723. & 0.0905 & 0.0623 \\
\hline 0.018951 & 0.019062 & 1.005701 & 0.000000 & 293.8 & 293.8 & 294.4 & 293.6 & 1.02 & 11769. & 0.0909 & 0.0623 \\
\hline 0.017200 & 0.017301 & 1.005701 & 0.000000 & 293.8 & 293.8 & 294.2 & 293.7 & 0.89 & 11820. & 0.0919 & 0.0623 \\
\hline 0.015048 & 0.015137 & 1.005701 & 0.000000 & 293.8 & 293.8 & 294.4 & 293.7 & 0.76 & 11885. & 0.0907 & 0.0623 \\
\hline 0.013818 & 0.013900 & 1.005701 & 0.000000 & 293.9 & 293.8 & 294.6 & 293.6 & 0.64 & 11937. & 0.0925 & 0.0623 \\
\hline 0.013441 & 0.013522 & 1.005701 & 0.000000 & 293.9 & 293.8 & 294.3 & 293.7 & 0.51 & 11982. & 0.0910 & 0.0623 \\
\hline 0.013486 & 0.013567 & 1.005701 & 0.000000 & 293.9 & 293.9 & 294.2 & 293.6 & 0.38 & 12028. & 0.0872 & 0.0623 \\
\hline 0.013593 & 0.013675 & 1.005701 & 0.000000 & 293.9 & 293.9 & 294.6 & 293.6 & 0.25 & 12073. & 0.0865 & 0.0623 \\
\hline 0.013592 & 0.013673 & 1.005701 & 0.000000 & 293.9 & 293.9 & 294.5 & 293.7 & 0.13 & 12118. & 0.0895 & 0.0623 \\
\hline 0.013199 & 0.013298 & 1.004021 & 0.000041 & 293.9 & 293.9 & 294.2 & 293.7 & 1.59 & 12289. & 0.0925 & 0.0623 \\
\hline 0.013292 & 0.013391 & 1.004021 & 0.000041 & 293.9 & 293.9 & 294.8 & 293.6 & 1.52 & 12336. & 0.0868 & 0.0623 \\
\hline 0.013225 & 0.013324 & 1.004021 & 0.000041 & 293.9 & 293.9 & 294.7 & 293.6 & 1.40 & 12382. & 0.0901 & 0.0623 \\
\hline 0.013196 & 0.013295 & 1.004021 & 0.000041 & 293.9 & 293.9 & 294.8 & 293.7 & 1.27 & 12433. & 0.0901 & 0.0623 \\
\hline
\end{tabular}


Table H.1.9 Diesel contamination on oxidized copper surface for $\operatorname{Re}=4000$ and $x_{\mathrm{b}}=0.3 \%$

\begin{tabular}{|c|c|c|c|c|c|c|c|c|c|c|c|}
\hline \multicolumn{12}{|c|}{$\begin{array}{l}\text { Diesel contamination on oxidized copper surface for } \mathrm{Re}=4000 \text { and } x_{\mathrm{b}}=0.3 \% \\
\text { (file:trv3con2.tbl) }\end{array}$} \\
\hline $\begin{array}{l}F \\
(\mathrm{v})\end{array}$ & $\begin{array}{l}F_{\mathrm{r}} \\
(\mathrm{v})\end{array}$ & $\begin{array}{c}F_{100} \\
(\mathrm{~V})\end{array}$ & $\begin{array}{l}F_{0} \\
(\mathrm{v})\end{array}$ & $\begin{array}{l}T_{\mathrm{Ti}} \\
(\mathrm{K})\end{array}$ & $\begin{array}{l}T_{\text {To }} \\
(\mathrm{K})\end{array}$ & $\begin{array}{c}T_{\mathrm{a}} \\
(\mathrm{K})\end{array}$ & $\begin{array}{c}T_{\mathrm{b}} \\
(\mathrm{K})\end{array}$ & $\begin{array}{c}l \\
(\mathrm{~mm})\end{array}$ & $\begin{array}{l}\text { Exposure } \\
\text { time (s) }\end{array}$ & $\begin{array}{c}\text { Turbine } \\
\text { meter } \\
\dot{m}_{\mathrm{w}}(\mathrm{kg} / \mathrm{s})\end{array}$ & 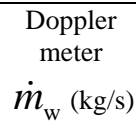 \\
\hline 0.011072 & 0.011157 & 1.004420 & 00.000033 & 293.9 & 293.9 & 293.9 & 293.7 & 1.59 & 64840. & 0.1899 & 0.1835 \\
\hline 0.010992 & 0.011077 & 1.004420 & 0.000033 & 293.9 & 293.9 & 293.9 & 293.7 & 1.52 & 64910. & 0.1952 & 0.1842 \\
\hline 0.010911 & 0.010995 & 1.004420 & 0.000033 & 293.9 & 293.9 & 293.9 & 293.7 & 1.40 & 64953. & 0.1946 & 0.1841 \\
\hline 0.010948 & 0.011031 & 1.004420 & 0.000033 & 293.8 & 293.8 & 293.9 & 293.7 & 1.27 & 65012. & 0.1887 & 0.1821 \\
\hline 0.010886 & 0.010968 & 1.004420 & 0.000033 & 293.8 & 293.8 & 293.9 & 293.7 & 1.14 & 65079. & 0.1922 & 0.1690 \\
\hline 0.010847 & 0.010929 & 1.004420 & 0.000033 & 293.8 & 293.8 & 293.9 & 293.7 & 1.02 & 65125. & 0.1927 & 0.1730 \\
\hline 0.010797 & 0.010878 & 1.004420 & 0.000033 & 293.8 & 293.8 & 293.9 & 293.7 & 0.89 & 65175. & 0.1940 & 0.1940 \\
\hline 0.010634 & 0.010714 & 1.004420 & 0.000033 & 293.8 & 293.8 & 294.1 & 293.7 & 0.76 & 65229. & 0.1934 & .2099 \\
\hline 0.010665 & 0.010745 & 1.004420 & 0.000033 & 293.7 & 293.7 & 293.9 & 293.7 & 0.64 & 65299. & 0.1983 & .2010 \\
\hline 0.010695 & 0.010775 & 1.004420 & 0.000033 & 293.7 & 293.7 & 294.0 & 293.7 & 0.51 & 65349. & 0.1922 & .1997 \\
\hline 0.010746 & 0.010825 & 1.004420 & 0.000033 & 293.6 & 293.6 & 294.0 & 293.7 & 0.38 & 65421. & 0.1910 & .2042 \\
\hline 0.010743 & 0.010821 & 1.004420 & 0.000033 & 293.6 & 293.6 & 293.9 & 293.7 & 0.25 & 65505. & 0.1977 & .1811 \\
\hline 0.010748 & 0.010825 & 1.004420 & 0.000033 & 293.5 & 293.5 & 293.9 & 293.7 & 0.13 & 65555. & 0.1887 & 1470 \\
\hline 0.011404 & 0.011431 & 1.003228 & -0.000015 & 294.0 & 293.9 & 293.9 & 293.7 & 1.59 & 68691. & 0.1910 & 1550 \\
\hline 0.011317 & 0.011343 & 1.003228 & -0.000015 & 293.9 & 293.9 & 293.9 & 293.7 & 1.52 & 68759. & 0.1958 & .1605 \\
\hline 0.011275 & 0.011301 & 1.003228 & -0.000015 & 293.9 & 293.9 & 293.9 & 293.7 & 1.40 & 68808. & 0.1989 & .1385 \\
\hline 0.011285 & 0.011311 & 1.003228 & -0.0000 & 293.9 & 293.9 & 293.9 & 293.7 & 1.27 & 68854. & 0.1910 & .1457 \\
\hline 0.011252 & 0.011278 & 1.003228 & -0.00001 & 293.9 & 293.9 & 293.9 & 293.7 & 1.14 & 68898. & 0.1853 & .1799 \\
\hline 0.011261 & 0.011286 & 1.003228 & -0.00001 & 293.9 & 293.9 & 294.0 & 293.7 & 1.02 & 68943. & 0.1964 & .1686 \\
\hline 0.011191 & 0.011215 & 1.003228 & -0.000015 & 293.8 & 293.8 & 294.0 & 293.7 & 0.89 & 69033. & 0.1934 & .1802 \\
\hline 0.011306 & 0.011331 & 1.003228 & -0.00001 & 293.8 & 293.8 & 293.9 & 293.7 & 0.76 & 69084. & 0.1893 & .1982 \\
\hline 0.011366 & 0.011390 & 1.003228 & -0.000015 & 293.8 & 293.8 & 293.9 & 293.7 & 0.64 & 69132. & 0.1940 & .1949 \\
\hline 0.011243 & 0.011266 & 1.003228 & -0.00001 & 293.8 & 293.8 & 293.9 & 293.7 & 0.51 & 69177. & 0.1916 & .1955 \\
\hline 0.011259 & 0.011282 & 1.003228 & -0.00001 & 293.8 & 293.7 & 294.0 & 293.7 & 0.38 & 69225. & 0.1843 & .2035 \\
\hline 0.011345 & 0.011368 & 1.003228 & -0.00001 & 293.7 & 293.7 & 293.9 & 293.7 & 0.25 & 69274. & 0.1922 & .2078 \\
\hline 0.011269 & 0.011292 & 1.003228 & -0.0000 & 293.7 & 293.7 & 294.0 & 293.7 & 0.13 & 69322. & 0.1910 & 0.2029 \\
\hline 0.011701 & 0.011696 & 1.006369 & -0.0000 & 293.9 & 293.9 & 294.1 & 293.7 & 1.59 & 72093. & 0.1922 & 0.1646 \\
\hline 0.011799 & 0.011796 & 1.006369 & -0.000084 & 293.9 & 293.9 & 294.1 & 293.7 & 1.52 & 72139. & 0.1946 & 0.1650 \\
\hline 0.011717 & 0.011713 & 1.006369 & -0.000084 & 293.9 & 293.9 & 294.1 & 293.7 & 1.40 & 72186. & 0.1945 & 0.1533 \\
\hline 0.011791 & 0.011788 & 1.006369 & -0.000084 & 293.9 & 293.9 & 294.1 & 293.7 & 1.27 & 72236. & 0.1893 & 0.1609 \\
\hline 0.011637 & 0.011633 & 1.006369 & -0.000084 & 294.0 & 293.9 & 294.1 & 293.7 & 1.14 & 72284. & 0.1951 & 0.1636 \\
\hline 0.011679 & 0.011675 & 1.006369 & -0.000084 & 294.0 & 293.9 & 294.1 & 293.7 & 1.02 & 72331. & 0.1952 & 0.1873 \\
\hline 0.011720 & 0.011717 & 1.006369 & -0.000084 & 294.0 & 293.9 & 294.1 & 293.7 & 0.89 & 72379. & 0.1853 & 0.2214 \\
\hline 0.011621 & 0.011617 & 1.006369 & -0.000084 & 294.0 & 294.0 & 294.1 & 293.7 & 0.76 & 72428. & 0.1927 & 0.2032 \\
\hline 0.011719 & 0.011716 & 1.006369 & -0.000084 & 294.0 & 293.9 & 294.1 & 293.7 & 0.64 & 72482. & 0.1865 & 0.1756 \\
\hline 0.011645 & 0.011641 & 1.006369 & -0.000084 & 294.0 & 293.9 & 294.0 & 293.7 & 0.51 & 72531. & 0.1945 & 0.1689 \\
\hline 0.011779 & 0.011776 & 1.006369 & -0.000084 & 294.0 & 294.0 & 294.0 & 293.7 & 0.38 & 72577. & 0.1881 & 0.1765 \\
\hline 0.011726 & 0.011723 & 1.006369 & -0.000084 & 293.9 & 293.9 & 294.0 & 293.7 & 0.25 & 72621. & 0.1977 & 0.1734 \\
\hline 0.011862 & 0.011860 & 1.006369 & -0.000084 & 293.9 & 293.9 & 294.0 & 293.7 & 0.13 & 72668. & 0.1904 & 0.1744 \\
\hline 0.012166 & 0.012227 & 1.008294 & -0.000045 & 294.0 & 293.9 & 294.1 & 293.7 & 1.59 & 76231. & 0.1945 & 0.1881 \\
\hline 0.012174 & 0.012236 & 1.008294 & -0.000045 & 294.0 & 293.9 & 294.1 & 293.7 & 1.52 & 76278. & 0.1922 & 0.1710 \\
\hline 0.012214 & 0.012276 & 1.008294 & -0.000045 & 294.0 & 293.9 & 294.1 & 293.7 & 1.40 & 76326. & 0.1837 & 0.1822 \\
\hline 0.012061 & 0.012121 & 1.008294 & -0.000045 & 294.0 & 294.0 & 294.1 & 293.7 & 1.27 & 76375. & 0.1898 & 0.1925 \\
\hline 0.012127 & 0.012188 & 1.008294 & -0.000045 & 294.0 & 293.9 & 294.1 & 293.7 & 1.14 & 76421. & 0.1910 & 0.1880 \\
\hline 0.012160 & 0.012222 & 1.008294 & -0.000045 & 294.0 & 293.9 & 294.1 & 293.7 & 1.02 & 76475. & 0.1934 & 0.1821 \\
\hline 0.012168 & 0.012230 & 1.008294 & -0.000045 & 294.0 & 294.0 & 294.2 & 293.7 & 0.89 & 76523. & 0.1934 & 0.1814 \\
\hline 0.012160 & 0.012222 & 1.008294 & -0.000045 & 294.0 & 293.9 & 294.1 & 293.7 & 0.76 & 76568. & 0.1853 & 0.1850 \\
\hline 0.012271 & 0.012333 & 1.008294 & -0.000045 & 294.0 & 293.9 & 294.1 & 293.7 & 0.64 & 76613. & 0.1859 & 0.1863 \\
\hline 0.012255 & 0.012318 & 1.008294 & -0.000045 & 294.0 & 293.9 & 294.2 & 293.7 & 0.51 & 76659. & 0.1934 & 0.1865 \\
\hline 0.012299 & 0.012361 & 1.008294 & -0.000045 & 294.0 & 293.9 & 294.1 & 293.7 & 0.38 & 76707. & 0.1983 & 0.1735 \\
\hline 0.012432 & 0.012495 & 1.008294 & -0.000045 & 293.9 & 293.9 & 294.1 & 293.7 & 0.25 & 76755. & 0.1983 & 0.1723 \\
\hline 0.012438 & 0.012501 & 1.008294 & -0.000045 & 293.9 & 293.9 & 294.2 & 293.7 & 0.13 & 76826. & 0.1934 & 0.1663 \\
\hline 0.012171 & 0.012131 & 1.004779 & -0.000097 & 293.6 & 293.5 & 294.3 & 293.7 & 1.59 & 79482. & 0.1952 & 0.1872 \\
\hline 0.012184 & 0.012144 & 1.004779 & -0.000097 & 293.6 & 293.5 & 294.3 & 293.7 & 1.52 & 79525. & 0.1899 & 0.1769 \\
\hline 0.012254 & 0.012216 & 1.004779 & -0.000097 & 293.6 & 293.6 & 294.2 & 293.7 & 1.40 & 79573. & 0.1910 & 0.1438 \\
\hline 0.012222 & 0.012183 & 1.004779 & -0.000097 & 293.6 & 293.6 & 294.2 & 293.7 & 1.27 & 79620. & 0.1848 & 0.1511 \\
\hline
\end{tabular}


Table H.1.10 Diesel contamination on oxidized copper surface for $\mathrm{Re}=5000$ and $x_{\mathrm{b}}=0.3 \%$

\begin{tabular}{|c|c|c|c|c|c|c|c|c|c|c|c|}
\hline \multicolumn{12}{|c|}{$\begin{array}{l}\text { Diesel contamination on oxidized copper surface for } \mathrm{Re}=5000 \text { and } x_{\mathrm{b}}=0.3 \% \\
\text { (file:trv45con2.tbl) }\end{array}$} \\
\hline $\begin{array}{l}F \\
(\mathrm{v})\end{array}$ & $\begin{array}{l}F_{\mathrm{r}} \\
(\mathrm{v})\end{array}$ & $\begin{array}{c}F_{100} \\
(\mathrm{v})\end{array}$ & $\begin{array}{l}F_{0} \\
(\mathrm{v})\end{array}$ & $\begin{array}{l}T_{\mathrm{Ti}} \\
(\mathrm{K})\end{array}$ & $\begin{array}{l}T_{\mathrm{To}} \\
(\mathrm{K})\end{array}$ & $\begin{array}{c}T_{\mathrm{a}} \\
(\mathrm{K})\end{array}$ & $\begin{array}{c}T_{\mathrm{b}} \\
(\mathrm{K})\end{array}$ & $\begin{array}{c}l \\
(\mathrm{~mm})\end{array}$ & $\begin{array}{l}\text { Exposure } \\
\text { time (s) }\end{array}$ & $\begin{array}{c}\text { Turbine } \\
\text { meter } \\
\dot{m}_{\mathrm{w}}(\mathrm{kg} / \mathrm{s})\end{array}$ & $\begin{array}{c}\text { Doppler } \\
\text { meter } \\
\dot{m}_{\mathrm{w}}(\mathrm{kg} / \mathrm{s})\end{array}$ \\
\hline 0.004208 & 0.004205 & 0.993235 & 00.000034 & 292.4 & 292.4 & 295.4 & 293.7 & 1.59 & 4061. & 0.2420 & 0.2266 \\
\hline 0.004008 & 0.004007 & 0.993235 & 0.000034 & 292.4 & 292.4 & 295.3 & 293.7 & 1.52 & 4103. & 0.2488 & 0.2322 \\
\hline 0.003698 & 0.003700 & 0.993235 & 0.000034 & 292.5 & 292.5 & 295.2 & 293.7 & 1.40 & 4144. & 0.2488 & 0.2378 \\
\hline 0.003431 & 0.003435 & 0.993235 & 0.000034 & 292.5 & 292.5 & 294.6 & 293.7 & 1.27 & 4187. & 0.2497 & 0.2440 \\
\hline 0.003121 & 0.003128 & 0.993235 & 0.000034 & 292.6 & 292.5 & 294.4 & 293.7 & 1.14 & 4229. & 0.2420 & 0.2478 \\
\hline 0.002796 & 0.002806 & 0.993235 & 0.000034 & 292.6 & 292.6 & 294.9 & 293.7 & 1.02 & 4271. & 0.2458 & 0.2502 \\
\hline 0.002473 & 0.002486 & 0.993235 & 0.000034 & 292.6 & 292.6 & 295.4 & 293.7 & 0.89 & 4315. & 0.2458 & 0.2462 \\
\hline 0.002210 & 0.002226 & 0.993235 & 0.000034 & 292.7 & 292.6 & 295.1 & 293.7 & 0.76 & 4360. & 0.2507 & 0.2210 \\
\hline 0.001853 & 0.001871 & 0.993235 & 0.000034 & 292.7 & 292.7 & 295.7 & 293.7 & 0.64 & 4402. & 0.2528 & 0.2271 \\
\hline 0.001530 & 0.001551 & 0.993235 & 0.000034 & 292.8 & 292.7 & 295.8 & 293.7 & 0.51 & 4444. & 0.2449 & 0.2448 \\
\hline 0.001180 & 0.001204 & 0.993235 & 0.000034 & 292.8 & 292.8 & 295.6 & 293.7 & 0.38 & 4488. & 0.2401 & 0.2488 \\
\hline 0.000923 & 0.000950 & 0.993235 & 0.000034 & 292.8 & 292.8 & 295.5 & 293.7 & 0.25 & 4530. & 0.2338 & 0.2444 \\
\hline 0.000681 & 0.000709 & 0.993235 & 0.000034 & 292.9 & 292.9 & 295.7 & 293.7 & 0.13 & 4574. & 0.2508 & 0.2383 \\
\hline 0.004110 & 0.004058 & 0.989872 & -0.000007 & 293.1 & 293.0 & 294.7 & 293.7 & 1.59 & 4733. & 0.2497 & 0.2248 \\
\hline 0.003916 & 0.003865 & 0.989872 & -0.000007 & 293.1 & 293.1 & 295.0 & 293.7 & 1.52 & 4776. & 0.2487 & 0.2322 \\
\hline 0.003659 & 0.003612 & 0.989872 & -0.000007 & 293.1 & 293.1 & 296.1 & 293.7 & 1.40 & 4819. & 0.2401 & 0.2489 \\
\hline 0.003347 & 0.003304 & 0.989872 & -0.000007 & 293.2 & 293.1 & 295.7 & 293.7 & 1.27 & 4862. & 0.2458 & 0.2416 \\
\hline 0.003064 & 0.003024 & 0.989872 & -0.000007 & 293.2 & 293.2 & 296.3 & 293.7 & 1.14 & 4903. & 0.2392 & 0.2417 \\
\hline 0.002810 & 0.002772 & 0.989872 & -0.000007 & 293.2 & 293.2 & 296.2 & 293.7 & 1.02 & 4948. & 0.2478 & 0.2329 \\
\hline 0.002424 & 0.002391 & 0.989872 & -0.000007 & 293.3 & 293.3 & 295.4 & 293.7 & 0.89 & 4990. & 0.2402 & 0.2246 \\
\hline 0.002123 & 0.002093 & 0.989872 & -0.000007 & 293.3 & 293.3 & 295.2 & 293.7 & 0.76 & 5032. & 0.2478 & 0.2041 \\
\hline 0.001808 & 0.001782 & 0.989872 & -0.000007 & 293.4 & 293.3 & 294.8 & 293.7 & 0.64 & 5077. & 0.2402 & 0.2124 \\
\hline 0.001516 & 0.001493 & 0.989872 & -0.000007 & 293.4 & 293.4 & 296.0 & 293.7 & 0.51 & 5121. & 0.2468 & 0.2318 \\
\hline 0.001248 & 0.001228 & 0.989872 & -0.000007 & 293.4 & 293.4 & 295.8 & 293.6 & 0.38 & 5163. & 0.2402 & 0.2284 \\
\hline 0.000887 & 0.000871 & 0.989872 & -0.000007 & 293.5 & 293.4 & 295.8 & 293.7 & 0.25 & 5205. & 0.2339 & 0.2381 \\
\hline 0.000559 & 0.000546 & 0.989872 & -0.000007 & 293.5 & 293.5 & 295.9 & 293.7 & 0.13 & 5247. & 0.2467 & 0.2513 \\
\hline 0.004139 & 0.004004 & 0.984469 & -0.000074 & 294.4 & 294.4 & 295.9 & 293.7 & 1.59 & 7217. & 0.2383 & 0.2167 \\
\hline 0.004077 & 0.003944 & 0.984469 & -0.000074 & 294.4 & 294.4 & 295.8 & 293.7 & 1.52 & 7260. & 0.2374 & 0.2182 \\
\hline 0.003855 & 0.003725 & 0.984469 & -0.000074 & 294.3 & 294.3 & 295.9 & 293.7 & 1.40 & 7312. & 0.2497 & 0.2093 \\
\hline 0.003594 & 0.003468 & 0.984469 & -0.000074 & 294.3 & 294.3 & 295.9 & 293.7 & 1.27 & 7363. & 0.2401 & 0.2119 \\
\hline 0.003310 & 0.003187 & 0.984469 & -0.000074 & 294.3 & 294.3 & 295.8 & 293.7 & 1.14 & 7418. & 0.2448 & 0.2173 \\
\hline 0.002939 & 0.002822 & 0.984469 & -0.000074 & 294.3 & 294.3 & 295.8 & 293.7 & 1.02 & 7466. & 0.2497 & 0.2195 \\
\hline 0.002658 & 0.002545 & 0.984469 & -0.000074 & 294.3 & 294.3 & 296.1 & 293.7 & 0.89 & 7510. & 0.2476 & 0.2221 \\
\hline 0.002330 & 0.002222 & 0.984469 & -0.000074 & 294.3 & 294.3 & 296.0 & 293.7 & 0.76 & 7553. & 0.2467 & 0.2251 \\
\hline 0.002069 & 0.001965 & 0.984469 & -0.000074 & 294.3 & 294.2 & 296.0 & 293.7 & 0.64 & 7596. & 0.2497 & 0.2678 \\
\hline 0.001724 & 0.001625 & 0.984469 & -0.000074 & 294.3 & 294.2 & 296.1 & 293.7 & 0.51 & 7638. & 0.2497 & 0.2355 \\
\hline 0.001494 & 0.001398 & 0.984469 & -0.000074 & 294.2 & 294.2 & 295.7 & 293.7 & 0.38 & 7682. & 0.2517 & 0.2429 \\
\hline 0.001162 & 0.001071 & 0.984469 & -0.000074 & 294.2 & 294.2 & 295.3 & 293.7 & 0.25 & 7724. & 0.2400 & 0.2493 \\
\hline 0.000913 & 0.000826 & 0.984469 & -0.000074 & 294.2 & 294.2 & 295.9 & 293.7 & 0.13 & 7767. & 0.2429 & 0.2530 \\
\hline 0.003987 & 0.004049 & 1.005697 & 0.000037 & 294.1 & 294.0 & 296.5 & 293.7 & 1.59 & 72772. & 0.2558 & 0.2324 \\
\hline 0.003930 & 0.003992 & 1.005697 & 0.000037 & 294.1 & 294.0 & 296.3 & 293.7 & 1.52 & 72816. & 0.2558 & 0.2511 \\
\hline 0.003774 & 0.003835 & 1.005697 & 0.000037 & 294.1 & 294.0 & 296.4 & 293.6 & 1.40 & 72863. & 0.2569 & 0.2579 \\
\hline 0.003574 & 0.003634 & 1.005697 & 0.000037 & 294.1 & 294.1 & 296.7 & 293.7 & 1.27 & 72904. & 0.2476 & 0.2651 \\
\hline 0.003399 & 0.003458 & 1.005697 & 0.000037 & 294.1 & 294.1 & 296.4 & 293.7 & 1.14 & 72945. & 0.2622 & 0.2697 \\
\hline 0.003093 & 0.003149 & 1.005697 & 0.000037 & 294.1 & 294.1 & 296.4 & 293.7 & 1.02 & 72988. & 0.2601 & 0.2501 \\
\hline 0.002719 & 0.002774 & 1.005697 & 0.000037 & 294.1 & 294.1 & 296.3 & 293.7 & 0.89 & 73030. & 0.2548 & 0.2617 \\
\hline 0.002359 & 0.002411 & 1.005697 & 0.000037 & 294.1 & 294.1 & 296.3 & 293.7 & 0.76 & 73071. & 0.2569 & 0.2594 \\
\hline 0.002145 & 0.002196 & 1.005697 & 0.000037 & 294.1 & 294.1 & 296.4 & 293.7 & 0.64 & 73115. & 0.2548 & 0.2626 \\
\hline 0.001927 & 0.001977 & 1.005697 & 0.000037 & 294.1 & 294.1 & 296.4 & 293.7 & 0.51 & 73157. & 0.2590 & 0.2634 \\
\hline 0.001635 & 0.001683 & 1.005697 & 0.000037 & 294.1 & 294.1 & 296.4 & 293.7 & 0.38 & 73202. & 0.2611 & 0.2676 \\
\hline 0.001386 & 0.001432 & 1.005697 & 0.000037 & 294.1 & 294.1 & 296.6 & 293.7 & 0.25 & 73245. & 0.2458 & 0.2695 \\
\hline 0.001104 & 0.001149 & 1.005697 & 0.000037 & 294.1 & 294.1 & 296.5 & 293.7 & 0.13 & 73286. & 0.2537 & 0.2679 \\
\hline 0.004063 & 0.004069 & 1.008256 & -0.000031 & 294.1 & 294.1 & 296.8 & 293.7 & 1.59 & 73421. & 0.2477 & 0.2603 \\
\hline 0.003791 & 0.003794 & 1.008256 & -0.000031 & 294.1 & 294.1 & 296.5 & 293.7 & 1.52 & 73462. & 0.2634 & 0.2560 \\
\hline 0.003890 & 0.003893 & 1.008256 & -0.000031 & 294.1 & 294.1 & 296.3 & 293.7 & 1.40 & 73506. & 0.2611 & 0.2613 \\
\hline 0.003280 & 0.003278 & 1.008256 & -0.000031 & 294.1 & 294.1 & 296.5 & 293.7 & 1.27 & 73548. & 0.2506 & 0.2641 \\
\hline 0.003152 & 0.003149 & 1.008256 & -0.000031 & 294.1 & 294.0 & 296.6 & 293.7 & 1.14 & 73591. & 0.2487 & 0.2619 \\
\hline
\end{tabular}


Table H.1.11 Diesel contamination on oxidized copper surface for $\mathrm{Re}=7000$ and $x_{\mathrm{b}}=0.3 \%$

\begin{tabular}{|c|c|c|c|c|c|c|c|c|c|c|c|}
\hline \multicolumn{12}{|c|}{$\begin{array}{l}\text { Diesel contamination on oxidized copper surface for } \mathrm{Re}=7000 \text { and } x_{\mathrm{b}}=0.3 \% \\
\text { (file:trv6con2.tbl) }\end{array}$} \\
\hline $\begin{array}{l}F \\
(\mathrm{v})\end{array}$ & $\begin{array}{c}F_{\mathrm{r}} \\
(\mathrm{v})\end{array}$ & $\begin{array}{c}F_{100} \\
(\mathrm{v})\end{array}$ & $\begin{array}{l}F_{0} \\
(\mathrm{v})\end{array}$ & $\begin{array}{l}T_{\mathrm{Ti}} \\
(\mathrm{K})\end{array}$ & $\begin{array}{l}T_{\mathrm{To}} \\
(\mathrm{K})\end{array}$ & $\begin{array}{c}T_{\mathrm{a}} \\
(\mathrm{K})\end{array}$ & $\begin{array}{c}T_{\mathrm{b}} \\
(\mathrm{K})\end{array}$ & $\begin{array}{c}l \\
(\mathrm{~mm})\end{array}$ & $\begin{array}{l}\text { Exposure } \\
\text { time (s) }\end{array}$ & $\begin{array}{c}\text { Turbine } \\
\text { meter } \\
\dot{m}_{\mathrm{w}}(\mathrm{kg} / \mathrm{s})\end{array}$ & $\begin{array}{c}\text { Doppler } \\
\text { meter } \\
\dot{m}_{\mathrm{w}}(\mathrm{kg} / \mathrm{s})\end{array}$ \\
\hline 0.013240 & 0.013403 & 1.004572 & 0.000105 & 293.6 & 293.6 & 294.5 & 293.7 & 1.59 & 63545. & 0.3372 & 0.0624 \\
\hline 0.015901 & 0.016076 & 1.004572 & 0.000105 & 293.6 & 293.6 & 294.5 & 293.7 & 1.52 & 63592. & 0.3354 & 0.0624 \\
\hline 0.013409 & 0.013573 & 1.004572 & 0.000105 & 293.6 & 293.6 & 294.6 & 293.7 & 1.40 & 63638. & 0.3372 & 0.3744 \\
\hline 0.010815 & 0.010968 & 1.004572 & 0.000105 & 293.7 & 293.6 & 294.5 & 293.7 & 1.27 & 63685. & 0.3198 & 0.0625 \\
\hline 0.012913 & 0.013076 & 1.004572 & 0.000105 & 293.7 & 293.7 & 294.5 & 293.7 & 1.14 & 63734. & 0.3182 & 0.0624 \\
\hline 0.009349 & 0.009496 & 1.004572 & 0.000105 & 293.7 & 293.7 & 294.5 & 293.7 & 1.02 & 63782. & 0.3150 & 0.0654 \\
\hline 0.009588 & 0.009737 & 1.004572 & 0.000105 & 293.8 & 293.7 & 294.6 & 293.7 & 0.89 & 63831. & 0.3265 & 0.0624 \\
\hline 0.007571 & 0.007712 & 1.004572 & 0.000105 & 293.8 & 293.8 & 294.6 & 293.7 & 0.76 & 63880. & 0.3248 & 0.0624 \\
\hline 0.006367 & 0.006503 & 1.004572 & 0.000105 & 293.8 & 293.8 & 294.6 & 293.7 & 0.64 & 63929. & 0.3389 & 0.0624 \\
\hline 0.006039 & 0.006174 & 1.004572 & 0.000105 & 293.9 & 293.8 & 294.5 & 293.6 & 0.51 & 63980. & 0.3102 & 0.0624 \\
\hline 0.004005 & 0.004130 & 1.004572 & 0.000105 & 293.9 & 293.9 & 294.6 & 293.7 & 0.38 & 64031. & 0.3299 & 0.3531 \\
\hline 0.003336 & 0.003458 & 1.004572 & 0.000105 & 293.9 & 293.9 & 294.6 & 293.6 & 0.25 & 64083. & 0.3407 & 0.0624 \\
\hline 0.004463 & 0.004590 & 1.004572 & 0.000105 & 293.9 & 293.9 & 294.5 & 293.6 & 0.13 & 64129. & 0.3198 & 0.0624 \\
\hline 0.014031 & 0.014287 & 1.009780 & 0.000126 & 293.5 & 293.5 & 294.6 & 293.7 & 1.59 & 67123. & 0.3246 & 0.0624 \\
\hline 0.015226 & 0.015493 & 1.009780 & 0.000126 & 293.5 & 293.5 & 294.6 & 293.7 & 1.52 & 67170. & 0.3248 & 0.0624 \\
\hline 0.011928 & 0.012165 & 1.009780 & 0.000126 & 293.4 & 293.4 & 294.6 & 293.7 & 1.40 & 67218. & 0.3231 & 0.0625 \\
\hline 0.011950 & 0.012186 & 1.009780 & 0.000126 & 293.4 & 293.4 & 294.6 & 293.7 & 1.27 & 67262. & 0.3353 & 0.3073 \\
\hline 0.013350 & 0.013599 & 1.009780 & 0.000126 & 293.4 & 293.4 & 294.6 & 293.7 & 1.14 & 67309. & 0.3353 & 0.0624 \\
\hline 0.009006 & 0.009215 & 1.009780 & 0.000126 & 293.4 & 293.4 & 294.6 & 293.7 & 1.02 & 67355. & 0.3246 & 0.0626 \\
\hline 0.009475 & 0.009688 & 1.009780 & 0.000126 & 293.4 & 293.4 & 294.5 & 293.7 & 0.89 & 67405. & 0.3371 & 0.0624 \\
\hline 0.007716 & 0.007913 & 1.009780 & 0.000126 & 293.4 & 293.4 & 294.4 & 293.7 & 0.76 & 67453. & 0.3281 & 0.0624 \\
\hline 0.006117 & 0.006299 & 1.009780 & 0.000126 & 293.4 & 293.4 & 294.4 & 293.7 & 0.64 & 67505. & 0.3182 & 0.0628 \\
\hline 0.005736 & 0.005914 & 1.009780 & 0.000126 & 293.4 & 293.4 & 294.5 & 293.7 & 0.51 & 67553. & 0.3317 & 0.3902 \\
\hline 0.004038 & 0.004201 & 1.009780 & 0.000126 & 293.4 & 293.4 & 294.3 & 293.7 & 0.38 & 67601. & 0.3317 & 0.3837 \\
\hline 0.003438 & 0.003595 & 1.009780 & 0.000126 & 293.4 & 293.4 & 294.4 & 293.7 & 0.25 & 67648. & 0.3133 & 0.0624 \\
\hline 0.002834 & 0.002986 & 1.009780 & 0.000126 & 293.4 & 293.4 & 294.4 & 293.7 & 0.13 & 67693. & 0.3389 & 0.3960 \\
\hline 0.006600 & 0.006756 & 1.007389 & 0.000107 & 293.7 & 293.7 & 294.2 & 293.7 & 1.59 & 71007. & 0.3183 & 0.3181 \\
\hline 0.006594 & 0.006749 & 1.007389 & 0.000107 & 293.6 & 293.6 & 294.1 & 293.7 & 1.52 & 71064. & 0.3486 & 0.3096 \\
\hline 0.006078 & 0.006229 & 1.007389 & 0.000107 & 293.6 & 293.6 & 294.2 & 293.7 & 1.40 & 71120. & 0.3267 & 0.3206 \\
\hline 0.005643 & 0.005791 & 1.007389 & 0.000107 & 293.6 & 293.6 & 294.0 & 293.7 & 1.27 & 71171. & 0.3392 & 0.3277 \\
\hline 0.005132 & 0.005276 & 1.007389 & 0.000107 & 293.6 & 293.6 & 294.1 & 293.7 & 1.14 & 71215. & 0.3430 & 0.3223 \\
\hline 0.004617 & 0.004757 & 1.007389 & 0.000107 & 293.5 & 293.5 & 294.1 & 293.7 & 1.02 & 71261. & 0.3251 & 0.3223 \\
\hline 0.004079 & 0.004215 & 1.007389 & 0.000107 & 293.5 & 293.5 & 294.2 & 293.7 & 0.89 & 71313. & 0.3392 & 0.3417 \\
\hline 0.003558 & 0.003690 & 1.007389 & 0.000107 & 293.5 & 293.5 & 294.4 & 293.7 & 0.76 & 71362. & 0.3234 & 0.3463 \\
\hline 0.003162 & 0.003291 & 1.007389 & 0.000107 & 293.5 & 293.5 & 294.1 & 293.7 & 0.64 & 71407. & 0.3320 & 0.3249 \\
\hline 0.002323 & 0.002447 & 1.007389 & 0.000107 & 293.5 & 293.5 & 294.0 & 293.7 & 0.51 & 71457. & 0.3354 & 0.3325 \\
\hline 0.002056 & 0.002178 & 1.007389 & 0.000107 & 293.4 & 293.4 & 294.4 & 293.7 & 0.38 & 71502. & 0.3320 & 0.3336 \\
\hline 0.001764 & 0.001884 & 1.007389 & 0.000107 & 293.4 & 293.4 & 294.1 & 293.7 & 0.25 & 71548. & 0.3392 & 0.3316 \\
\hline 0.001366 & 0.001482 & 1.007389 & 0.000107 & 293.4 & 293.4 & 294.4 & 293.7 & 0.13 & 71593. & 0.3217 & 0.3290 \\
\hline 0.006422 & 0.006540 & 1.005913 & 0.000077 & 294.0 & 294.0 & 294.3 & 293.7 & 1.59 & 74448. & 0.3392 & 0.3111 \\
\hline 0.006279 & 0.006396 & 1.005913 & 0.000077 & 294.0 & 294.0 & 294.1 & 293.7 & 1.52 & 74495. & 0.3185 & 0.3107 \\
\hline 0.005683 & 0.005796 & 1.005913 & 0.000077 & 294.0 & 294.0 & 294.0 & 293.7 & 1.40 & 74538. & 0.3429 & 0.2996 \\
\hline 0.005293 & 0.005404 & 1.005913 & 0.000077 & 294.0 & 294.0 & 294.2 & 293.7 & 1.27 & 74584. & 0.3392 & 0.3098 \\
\hline 0.004710 & 0.004817 & 1.005913 & 0.000077 & 294.0 & 293.9 & 294.3 & 293.7 & 1.14 & 74629. & 0.3338 & 0.3120 \\
\hline 0.004242 & 0.004345 & 1.005913 & 0.000077 & 294.0 & 293.9 & 294.2 & 293.7 & 1.02 & 74679. & 0.3447 & 0.3138 \\
\hline 0.003850 & 0.003951 & 1.005913 & 0.000077 & 293.9 & 293.9 & 294.1 & 293.7 & 0.89 & 74725. & 0.3268 & 0.3178 \\
\hline 0.003439 & 0.003537 & 1.005913 & 0.000077 & 293.9 & 293.9 & 294.4 & 293.7 & 0.76 & 74768. & 0.3374 & 0.3251 \\
\hline 0.002879 & 0.002974 & 1.005913 & 0.000077 & 293.9 & 293.9 & 294.4 & 293.7 & 0.64 & 74816. & 0.3410 & 0.3271 \\
\hline 0.002648 & 0.002741 & 1.005913 & 0.000077 & 293.9 & 293.9 & 294.1 & 293.7 & 0.51 & 74862. & 0.3429 & 0.3340 \\
\hline 0.002338 & 0.002429 & 1.005913 & 0.000077 & 293.9 & 293.9 & 294.0 & 293.7 & 0.38 & 74908. & 0.3410 & 0.3447 \\
\hline 0.002140 & 0.002230 & 1.005913 & 0.000077 & 293.8 & 293.8 & 294.4 & 293.7 & 0.25 & 74956. & 0.3268 & 0.3455 \\
\hline 0.002386 & 0.002477 & 1.005913 & 0.000077 & 293.8 & 293.8 & 294.3 & 293.7 & 0.13 & 75002. & 0.3320 & 0.3456 \\
\hline 0.004443 & 0.004531 & 1.014729 & 0.000021 & 293.9 & 293.9 & 294.3 & 293.7 & 1.59 & 79055. & 0.3302 & 0.3190 \\
\hline 0.004224 & 0.004308 & 1.014729 & 0.000021 & 293.9 & 293.9 & 294.4 & 293.7 & 1.52 & 79101. & 0.3485 & 0.3097 \\
\hline 0.003983 & 0.004064 & 1.014729 & 0.000021 & 293.9 & 293.9 & 294.4 & 293.7 & 1.40 & 79145. & 0.3216 & 0.3186 \\
\hline 0.003816 & 0.003894 & 1.014729 & 0.000021 & 293.9 & 293.8 & 294.3 & 293.7 & 1.27 & 79190. & 0.3391 & 0.3295 \\
\hline 0.003562 & 0.003636 & 1.014729 & 0.000021 & 293.8 & 293.8 & 294.3 & 293.7 & 1.14 & 79238. & 0.3267 & 0.3324 \\
\hline
\end{tabular}


Table H.1.12 Tap water flushing after $\mathrm{Re}=5000$ contamination tests at $x_{\mathrm{b}}=0.3 \%$

\begin{tabular}{|c|c|c|c|c|c|c|c|c|c|c|c|}
\hline \multicolumn{12}{|c|}{ Tap water flushing after Re $=5000$ contamination tests at $x_{\mathrm{b}}=0.3 \%$ (file:flsh45c2.tbl) } \\
\hline $\begin{array}{c}F \\
(\mathrm{v})\end{array}$ & $\begin{array}{c}F_{\mathrm{r}} \\
(\mathrm{v})\end{array}$ & $\begin{array}{c}F_{100} \\
(\mathrm{v})\end{array}$ & $\begin{array}{l}F_{0} \\
(\mathrm{v})\end{array}$ & $\begin{array}{l}T_{\mathrm{Ti}} \\
(\mathrm{K})\end{array}$ & $\begin{array}{l}T_{\mathrm{To}} \\
(\mathrm{K})\end{array}$ & $\begin{array}{c}T_{\mathrm{a}} \\
(\mathrm{K})\end{array}$ & $\begin{array}{c}T_{\mathrm{b}} \\
(\mathrm{K})\end{array}$ & $\begin{array}{c}l \\
(\mathrm{~mm})\end{array}$ & $\begin{array}{c}\text { Exposure } \\
\text { time (s) }\end{array}$ & $\begin{array}{c}\text { Turbine } \\
\text { meter } \\
\dot{m}_{\mathrm{w}}(\mathrm{kg} / \mathrm{s})\end{array}$ & 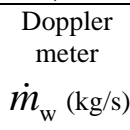 \\
\hline 00.000048 & 00.000113 & 1.007885 & 00.000065 & 293.3 & 293.1 & 297.0 & 293.7 & 1.59 & 402. & N/A & N/A \\
\hline-0.000054 & 0.000010 & 1.007885 & 0.000065 & 292.7 & 292.5 & 297.2 & 293.7 & 1.52 & 453. & N/A & N/A \\
\hline 0.000103 & 0.000169 & 1.007885 & 0.000065 & 292.1 & 291.9 & 297.1 & 293.7 & 1.40 & 506. & N/A & N/A \\
\hline 0.000066 & 0.000131 & 1.007885 & 0.000065 & 291.6 & 291.4 & 297.0 & 293.7 & 1.27 & 557. & N/A & N/A \\
\hline 0.000034 & 0.000099 & 1.007885 & 0.000065 & 291.1 & 290.9 & 297.0 & 293.7 & 1.14 & 610. & N/A & N/A \\
\hline 0.000180 & 0.000245 & 1.007885 & 0.000065 & 290.6 & 290.5 & 296.9 & 293.7 & 1.02 & 663. & N/A & N/A \\
\hline 0.000047 & 0.000111 & 1.007885 & 0.000065 & 290.2 & 290.0 & 296.9 & 293.7 & 0.89 & 719. & N/A & N/A \\
\hline 0.000019 & 0.000083 & 1.007885 & 0.000065 & 289.7 & 289.5 & 296.7 & 293.7 & 0.76 & 775. & N/A & N/A \\
\hline 0.000044 & 0.000108 & 1.007885 & 0.000065 & 289.3 & 289.1 & 296.8 & 293.7 & 0.64 & 832. & N/A & N/A \\
\hline 0.000029 & 0.000093 & 1.007885 & 0.000065 & 289.0 & 288.8 & 296.9 & 293.7 & 0.51 & 886. & N/A & N/A \\
\hline 0.000113 & 0.000177 & 1.007885 & 0.000065 & 288.7 & 288.6 & 296.9 & 293.7 & 0.38 & 938. & N/A & N/A \\
\hline 0.000299 & 0.000363 & 1.007885 & 0.000065 & 288.5 & 288.4 & 297.0 & 293.7 & 0.25 & 995. & N/A & N/A \\
\hline 0.000638 & 0.000702 & 1.007885 & 0.000065 & 288.4 & 288.3 & 297.0 & 293.7 & 0.13 & 1053. & N/A & N/A \\
\hline 0.000061 & 0.000048 & 1.006992 & -0.000012 & 286.4 & 286.4 & 297.0 & 293.7 & 1.59 & 3736. & N/A & N/A \\
\hline-0.000004 & -0.000016 & 1.006992 & -0.000012 & 286.4 & 286.4 & 296.8 & 293.7 & 1.52 & 3787. & N/A & N/A \\
\hline 0.000019 & 0.000006 & 1.006992 & -0.000012 & 286.4 & 286.4 & 297.0 & 293.7 & 1.40 & 3842. & N/A & N/A \\
\hline 0.000000 & -0.000012 & 1.006992 & -0.000012 & 286.4 & 286.4 & 297.2 & 293.7 & 1.27 & 3902. & N/A & N/A \\
\hline 0.000025 & 0.000013 & 1.006992 & -0.000012 & 286.4 & 286.3 & 297.1 & 293.7 & 1.14 & 3958. & N/A & N/A \\
\hline 0.000029 & 0.000016 & 1.006992 & -0.000012 & 286.4 & 286.4 & 297.0 & 293.7 & 1.02 & 4013. & N/A & N/A \\
\hline 0.000017 & 0.000005 & 1.006992 & -0.000012 & 286.4 & 286.4 & 297.0 & 293.7 & 0.89 & 4070. & N/A & N/A \\
\hline 0.000038 & 0.000026 & 1.006992 & -0.000012 & 286.4 & 286.4 & 297.1 & 293.7 & 0.76 & 4124. & N/A & N/A \\
\hline 0.000029 & 0.000016 & 1.006992 & -0.000012 & 286.4 & 286.4 & 297.4 & 293.7 & 0.64 & 4180. & N/A & N/A \\
\hline 0.000049 & 0.000037 & 1.006992 & -0.000012 & 286.4 & 286.4 & 297.0 & 293.7 & 0.51 & 4234. & N/A & N/A \\
\hline 0.000018 & 0.000006 & 1.006992 & -0.000012 & 286.4 & 286.3 & 297.0 & 293.7 & 0.38 & 4294. & N/A & N/A \\
\hline 0.000023 & 0.000011 & 1.006992 & -0.000012 & 286.4 & 286.4 & 297.2 & 293.7 & 0.25 & 4349. & N/A & N/A \\
\hline-0.000002 & -0.000014 & 1.006992 & -0.000012 & 286.4 & 286.3 & 297.3 & 293.7 & 0.13 & 4406. & N/A & N/A \\
\hline-0.000262 & -0.000424 & 1.006313 & -0.000164 & 287.3 & 287.3 & 295.0 & 293.7 & 1.59 & 64014. & N/A & N/A \\
\hline-0.000287 & -0.000449 & 1.006313 & -0.000164 & 287.3 & 287.3 & 295.0 & 293.7 & 1.52 & 64064. & N/A & N/A \\
\hline-0.000282 & -0.000443 & 1.006313 & -0.000164 & 287.3 & 287.3 & 295.1 & 293.6 & 1.40 & 64118. & N/A & N/A \\
\hline-0.000297 & -0.000459 & 1.006313 & -0.000164 & 287.3 & 287.3 & 295.0 & 293.7 & 1.27 & 64176. & N/A & N/A \\
\hline-0.000306 & -0.000468 & 1.006313 & -0.000164 & 287.3 & 287.3 & 295.0 & 293.7 & 1.14 & 64239. & N/A & N/A \\
\hline-0.000310 & -0.000471 & 1.006313 & -0.000164 & 287.3 & 287.3 & 295.0 & 293.7 & 1.02 & 64292. & N/A & N/A \\
\hline-0.000308 & -0.000469 & 1.006313 & -0.000164 & 287.3 & 287.3 & 295.1 & 293.7 & 0.89 & 64346. & N/A & N/A \\
\hline-0.000289 & -0.000451 & 1.006313 & -0.000164 & 287.3 & 287.3 & 295.1 & 293.7 & 0.76 & 64398. & N/A & N/A \\
\hline-0.000298 & -0.000460 & 1.006313 & -0.000164 & 287.3 & 287.3 & 295.3 & 293.7 & 0.64 & 64452. & N/A & N/A \\
\hline-0.000302 & -0.000464 & 1.006313 & -0.000164 & 287.3 & 287.3 & 295.4 & 293.6 & 0.51 & 64508. & N/A & N/A \\
\hline-0.000305 & -0.000467 & 1.006313 & -0.000164 & 287.3 & 287.3 & 295.1 & 293.7 & 0.38 & 64560. & N/A & N/A \\
\hline-0.000313 & -0.000474 & 1.006313 & -0.000164 & 287.3 & 287.3 & 295.2 & 293.7 & 0.25 & 64612. & N/A & N/A \\
\hline-0.000311 & -0.000472 & 1.006313 & -0.000164 & 287.3 & 287.3 & 295.1 & 293.7 & 0.13 & 64667. & N/A & N/A \\
\hline-0.000130 & -0.000221 & 1.003946 & -0.000093 & 287.4 & 287.3 & 295.3 & 293.7 & 1.59 & 64866. & N/A & N/A \\
\hline-0.000142 & -0.000233 & 1.003946 & -0.000093 & 287.4 & 287.3 & 295.0 & 293.7 & 1.52 & 64920. & N/A & N/A \\
\hline-0.000145 & -0.000237 & 1.003946 & -0.000093 & 287.4 & 287.3 & 295.3 & 293.7 & 1.40 & 64975. & N/A & N/A \\
\hline-0.000119 & -0.000211 & 1.003946 & -0.000093 & 287.4 & 287.3 & 295.3 & 293.7 & 1.27 & 65027. & N/A & N/A \\
\hline-0.000142 & -0.000234 & 1.003946 & -0.000093 & 287.4 & 287.3 & 295.1 & 293.7 & 1.14 & 65082. & N/A & N/A \\
\hline-0.000162 & -0.000253 & 1.003946 & -0.000093 & 287.4 & 287.3 & 295.1 & 293.7 & 1.02 & 65135. & N/A & N/A \\
\hline-0.000138 & -0.000229 & 1.003946 & -0.000093 & 287.4 & 287.3 & 295.0 & 293.6 & 0.89 & 65188. & N/A & N/A \\
\hline-0.000132 & -0.000224 & 1.003946 & -0.000093 & 287.4 & 287.3 & 295.3 & 293.7 & 0.76 & 65245. & N/A & N/A \\
\hline-0.000127 & -0.000218 & 1.003946 & -0.000093 & 287.4 & 287.3 & 295.2 & 293.6 & 0.64 & 65300. & N/A & N/A \\
\hline-0.000144 & -0.000235 & 1.003946 & -0.000093 & 287.4 & 287.3 & 295.3 & 293.7 & 0.51 & 65351. & N/A & N/A \\
\hline-0.000139 & -0.000230 & 1.003946 & -0.000093 & 287.4 & 287.3 & 295.2 & 293.6 & 0.38 & 65405. & N/A & N/A \\
\hline-0.000136 & -0.000227 & 1.003946 & -0.000093 & 287.4 & 287.3 & 295.1 & 293.7 & 0.25 & 65458. & N/A & N/A \\
\hline-0.000135 & -0.000226 & 1.003946 & -0.000093 & 287.4 & 287.3 & 295.4 & 293.7 & 0.13 & 65513. & N/A & N/A \\
\hline-0.000254 & -0.000184 & 1.008369 & 0.000071 & 287.5 & 287.5 & 295.2 & 293.7 & 1.59 & 67977. & N/A & N/A \\
\hline-0.000289 & -0.000218 & 1.008369 & 0.000071 & 287.5 & 287.5 & 295.3 & 293.7 & 1.52 & 68028. & N/A & N/A \\
\hline-0.000286 & -0.000216 & 1.008369 & 0.000071 & 287.5 & 287.5 & 295.4 & 293.7 & 1.40 & 68086. & N/A & N/A \\
\hline-0.000299 & -0.000228 & 1.008369 & 0.000071 & 287.5 & 287.5 & 295.2 & 293.7 & 1.27 & 68139. & N/A & N/A \\
\hline-0.000294 & -0.000224 & 1.008369 & 0.000071 & 287.5 & 287.5 & 295.2 & 293.7 & 1.14 & 68190. & N/A & N/A \\
\hline-0.000283 & -0.000213 & 1.008369 & 0.000071 & 287.5 & 287.5 & 295.1 & 293.7 & 1.02 & 68242. & N/A & N/A \\
\hline
\end{tabular}


Table H.1.13 Tap water flushing after $\mathrm{Re}=7000$ contamination tests at $x_{\mathrm{b}}=0.3 \%$

\begin{tabular}{|c|c|c|c|c|c|c|c|c|c|c|c|}
\hline \multicolumn{12}{|c|}{ Tap water flushing after $\mathrm{Re}=7000$ contamination tests at $x_{\mathrm{b}}=0.3 \%$ (file:flsh6c2.tbl) } \\
\hline $\begin{array}{c}F \\
(\mathrm{v})\end{array}$ & $\begin{array}{c}F_{\mathrm{r}} \\
(\mathrm{v})\end{array}$ & $\begin{array}{c}F_{100} \\
(\mathrm{v})\end{array}$ & $\begin{array}{l}F_{0} \\
(\mathrm{v})\end{array}$ & $\begin{array}{l}T_{\mathrm{Ti}} \\
(\mathrm{K})\end{array}$ & $\begin{array}{l}T_{\mathrm{To}} \\
(\mathrm{K})\end{array}$ & $\begin{array}{c}T_{\mathrm{a}} \\
(\mathrm{K})\end{array}$ & $\begin{array}{c}T_{\mathrm{b}} \\
(\mathrm{K})\end{array}$ & $\begin{array}{c}l \\
(\mathrm{~mm})\end{array}$ & $\begin{array}{c}\text { Exposure } \\
\text { time (s) }\end{array}$ & $\begin{array}{c}\text { Turbine } \\
\text { meter } \\
\dot{m}_{\mathrm{w}}(\mathrm{kg} / \mathrm{s})\end{array}$ & $\begin{array}{c}\text { Doppler } \\
\text { meter } \\
\dot{m}_{\mathrm{w}}(\mathrm{kg} / \mathrm{s})\end{array}$ \\
\hline $\begin{array}{c}- \\
00.000236\end{array}$ & $\begin{array}{c}- \\
00.000174\end{array}$ & 1.005803 & 00.000064 & 296.9 & 296.8 & 295.4 & 293.7 & 1.59 & 0 . & N/A & N/A \\
\hline-0.000369 & -0.000308 & 1.005803 & 0.000064 & 296.0 & 295.9 & 295.4 & 293.7 & 1.52 & 56. & N/A & N/A \\
\hline-0.000370 & -0.000309 & 1.005803 & 0.000064 & 295.5 & 295.4 & 295.4 & 293.7 & 1.40 & 110. & N/A & N/A \\
\hline-0.000406 & -0.000345 & 1.005803 & 0.000064 & 294.9 & 294.8 & 295.4 & 293.7 & 1.27 & 165. & N/A & N/A \\
\hline-0.000409 & -0.000347 & 1.005803 & 0.000064 & 294.3 & 294.1 & 295.4 & 293.7 & 1.14 & 218. & N/A & N/A \\
\hline-0.000421 & -0.000359 & 1.005803 & 0.000064 & 293.6 & 293.5 & 295.4 & 293.7 & 1.02 & 276. & N/A & N/A \\
\hline-0.000425 & -0.000363 & 1.005803 & 0.000064 & 293.0 & 292.8 & 295.4 & 293.7 & 0.89 & 328. & N/A & N/A \\
\hline-0.000423 & -0.000360 & 1.005803 & 0.000064 & 292.1 & 291.8 & 295.4 & 293.7 & 0.76 & 383. & N/A & N/A \\
\hline-0.000438 & -0.000375 & 1.005803 & 0.000064 & 290.9 & 290.7 & 295.4 & 293.7 & 0.64 & 439. & N/A & N/A \\
\hline-0.000438 & -0.000375 & 1.005803 & 0.000064 & 290.0 & 289.7 & 295.4 & 293.7 & 0.51 & 492. & N/A & N/A \\
\hline-0.000453 & -0.000389 & 1.005803 & 0.000064 & 289.2 & 288.9 & 295.4 & 293.7 & 0.38 & 544. & N/A & N/A \\
\hline-0.000411 & -0.000347 & 1.005803 & 0.000064 & 288.5 & 288.3 & 295.4 & 293.7 & 0.25 & 600. & N/A & N/A \\
\hline-0.000442 & -0.000377 & 1.005803 & 0.000064 & 288.0 & 287.7 & 295.4 & 293.7 & 0.13 & 654. & N/A & N/A \\
\hline 0.000007 & 0.000068 & 1.010679 & 0.000062 & 284.3 & 284.2 & 296.1 & 293.7 & 1.59 & 11606. & N/A & N/A \\
\hline-0.000037 & 0.000025 & 1.010679 & 0.000062 & 284.3 & 284.2 & 296.1 & 293.7 & 1.52 & 11662. & N/A & N/A \\
\hline-0.000023 & 0.000038 & 1.010679 & 0.000062 & 284.3 & 284.2 & 296.0 & 293.7 & 1.40 & 11753. & N/A & N/A \\
\hline-0.000025 & 0.000036 & 1.010679 & 0.000062 & 284.2 & 284.2 & 296.0 & 293.7 & 1.27 & 11809. & N/A & N/A \\
\hline-0.000035 & 0.000026 & 1.010679 & 0.000062 & 284.2 & 284.2 & 296.1 & 293.7 & 1.14 & 11862. & N/A & N/A \\
\hline-0.000033 & 0.000029 & 1.010679 & 0.000062 & 284.2 & 284.2 & 296.0 & 293.7 & 1.02 & 11918. & N/A & N/A \\
\hline 0.000006 & 0.000067 & 1.010679 & 0.000062 & 284.2 & 284.1 & 296.1 & 293.7 & 0.89 & 11974. & N/A & N/A \\
\hline-0.000007 & 0.000054 & 1.010679 & 0.000062 & 284.2 & 284.2 & 296.1 & 293.7 & 0.76 & 12030. & N/A & N/A \\
\hline-0.000025 & 0.000036 & 1.010679 & 0.000062 & 284.2 & 284.1 & 296.1 & 293.7 & 0.64 & 12091. & N/A & N/A \\
\hline 0.000000 & 0.000061 & 1.010679 & 0.000062 & 284.2 & 284.1 & 296.1 & 293.7 & 0.51 & 12143. & N/A & N/A \\
\hline-0.000012 & 0.000050 & 1.010679 & 0.000062 & 284.2 & 284.1 & 296.1 & 293.7 & 0.38 & 12197. & N/A & N/A \\
\hline-0.000037 & 0.000024 & 1.010679 & 0.000062 & 284.2 & 284.1 & 296.1 & 293.7 & 0.25 & 12250. & N/A & N/A \\
\hline-0.000042 & 0.000019 & 1.010679 & 0.000062 & 284.2 & 284.1 & 296.1 & 293.7 & 0.13 & 12305. & N/A & N/A \\
\hline-0.000055 & 0.000002 & 1.003075 & 0.000057 & 283.7 & 283.6 & 295.6 & 293.7 & 1.59 & 15484. & N/A & N/A \\
\hline-0.000104 & -0.000047 & 1.003075 & 0.000057 & 283.7 & 283.7 & 295.8 & 293.7 & 1.52 & 15541. & N/A & N/A \\
\hline-0.000149 & -0.000091 & 1.003075 & 0.000057 & 283.7 & 283.7 & 295.7 & 293.7 & 1.40 & 15599. & N/A & N/A \\
\hline-0.000141 & -0.000084 & 1.003075 & 0.000057 & 283.7 & 283.6 & 295.7 & 293.7 & 1.27 & 15652. & N/A & N/A \\
\hline-0.000123 & -0.000066 & 1.003075 & 0.000057 & 283.7 & 283.7 & 295.4 & 293.7 & 1.14 & 15722. & N/A & N/A \\
\hline-0.000129 & -0.000072 & 1.003075 & 0.000057 & 283.7 & 283.7 & 295.5 & 293.7 & 1.02 & 15778. & N/A & N/A \\
\hline-0.000098 & -0.000041 & 1.003075 & 0.000057 & 283.7 & 283.7 & 295.7 & 293.7 & 0.89 & 15835. & N/A & N/A \\
\hline-0.000129 & -0.000072 & 1.003075 & 0.000057 & 283.8 & 283.7 & 295.8 & 293.7 & 0.76 & 15890. & N/A & N/A \\
\hline-0.000126 & -0.000069 & 1.003075 & 0.000057 & 283.7 & 283.7 & 295.3 & 293.7 & 0.64 & 15953. & N/A & N/A \\
\hline-0.000152 & -0.000094 & 1.003075 & 0.000057 & 283.7 & 283.6 & 295.2 & 293.7 & 0.51 & 16008. & N/A & N/A \\
\hline-0.000127 & -0.000070 & 1.003075 & 0.000057 & 283.7 & 283.6 & 295.7 & 293.7 & 0.38 & 16065. & N/A & N/A \\
\hline-0.000187 & -0.000129 & 1.003075 & 0.000057 & 283.7 & 283.7 & 295.4 & 293.7 & 0.25 & 16120. & N/A & N/A \\
\hline-0.000173 & -0.000115 & 1.003075 & 0.000057 & 283.7 & 283.6 & 295.4 & 293.7 & 0.13 & 16191. & N/A & N/A \\
\hline-0.000298 & -0.000403 & 1.005909 & -0.000108 & 285.3 & 285.3 & 294.0 & 293.7 & 1.59 & 77260. & N/A & N/A \\
\hline-0.000334 & -0.000438 & 1.005909 & -0.000108 & 285.3 & 285.3 & 294.0 & 293.7 & 1.52 & 77315. & N/A & N/A \\
\hline-0.000319 & -0.000423 & 1.005909 & -0.000108 & 285.3 & 285.3 & 294.2 & 293.7 & 1.40 & 77369. & N/A & N/A \\
\hline-0.000357 & -0.000461 & 1.005909 & -0.000108 & 285.3 & 285.3 & 294.1 & 293.7 & 1.27 & 77421. & N/A & N/A \\
\hline-0.000316 & -0.000421 & 1.005909 & -0.000108 & 285.3 & 285.3 & 294.0 & 293.7 & 1.14 & 77475. & N/A & N/A \\
\hline-0.000364 & -0.000468 & 1.005909 & -0.000108 & 285.3 & 285.3 & 293.9 & 293.7 & 1.02 & 77526. & N/A & N/A \\
\hline-0.000340 & -0.000444 & 1.005909 & -0.000108 & 285.3 & 285.3 & 294.0 & 293.7 & 0.89 & 77580. & N/A & N/A \\
\hline-0.000316 & -0.000420 & 1.005909 & -0.000108 & 285.3 & 285.3 & 294.0 & 293.7 & 0.76 & 77632. & N/A & N/A \\
\hline-0.000324 & -0.000429 & 1.005909 & -0.000108 & 285.3 & 285.3 & 294.1 & 293.7 & 0.64 & 77682. & N/A & N/A \\
\hline-0.000313 & -0.000417 & 1.005909 & -0.000108 & 285.3 & 285.3 & 293.9 & 293.7 & 0.51 & 77735. & N/A & N/A \\
\hline-0.000346 & -0.000450 & 1.005909 & -0.000108 & 285.3 & 285.3 & 294.0 & 293.7 & 0.38 & 77789. & N/A & N/A \\
\hline-0.000366 & -0.000470 & 1.005909 & -0.000108 & 285.3 & 285.3 & 294.0 & 293.7 & 0.25 & 77841. & N/A & N/A \\
\hline-0.000355 & -0.000458 & 1.005909 & -0.000108 & 285.3 & 285.3 & 293.9 & 293.7 & 0.13 & 77895. & N/A & N/A \\
\hline-0.000385 & -0.000396 & 1.004326 & -0.000015 & 285.3 & 285.3 & 294.0 & 293.7 & 1.59 & 78063. & N/A & N/A \\
\hline-0.000418 & -0.000429 & 1.004326 & -0.000015 & 285.3 & 285.3 & 294.0 & 293.7 & 1.52 & 78114. & N/A & N/A \\
\hline-0.000463 & -0.000474 & 1.004326 & -0.000015 & 285.3 & 285.2 & 293.9 & 293.7 & 1.40 & 78168. & N/A & N/A \\
\hline-0.000453 & -0.000463 & 1.004326 & -0.000015 & 285.3 & 285.2 & 294.0 & 293.7 & 1.27 & 78219. & N/A & N/A \\
\hline-0.000406 & -0.000417 & 1.004326 & -0.000015 & 285.2 & 285.2 & 293.9 & 293.7 & 1.14 & 78274. & N/A & N/A \\
\hline
\end{tabular}


Table H.2.1 Diesel contamination on oxidized copper surface for $\mathrm{Re}=0$ and $x_{\mathrm{b}}=0.2 \%$ (file:trv0con1.tb2)

\begin{tabular}{|c|c|c|c|c|c|c|c|}
\hline $\begin{array}{c}l_{\mathrm{e}} \\
(\mu \mathrm{m})\end{array}$ & $\begin{array}{c}\Gamma \\
\left(\mathrm{kg} / \mathrm{m}^{2}\right) \\
\mathrm{X} 10^{5}\end{array}$ & $\begin{array}{l}x_{\mathrm{b}} \\
(\%)\end{array}$ & $\begin{array}{c}\text { Exposure } \\
\text { Time (s) }\end{array}$ & Re & $\begin{array}{l}\bar{T}_{\mathrm{T}_{\mathrm{T}}} \\
(\mathrm{K})\end{array}$ & $\begin{array}{c}T_{\mathrm{b}}-\bar{T}_{\mathrm{T}_{\mathrm{T}}} \\
(\mathrm{K})\end{array}$ & $\frac{F_{T_{\mathrm{b}}}}{F_{T_{\mathrm{T}}}}$ \\
\hline 2.15 & 183. & $\begin{array}{c}- \\
0.002\end{array}$ & 792. & 0. & 294.4 & -0.8 & 1.00 \\
\hline 2.15 & 183. & $\begin{array}{c}- \\
0.002\end{array}$ & 842. & 0. & 294.4 & $\begin{array}{l}-0.8 \\
\end{array}$ & 1.00 \\
\hline 2.15 & 183. & $\overline{-}$ & 894. & 0. & 294.4 & -0.9 & 1.00 \\
\hline 2.15 & 183. & $\begin{array}{c}- \\
0.002\end{array}$ & 945. & 0. & 294.5 & -0.9 & 1.00 \\
\hline 2.15 & 183. & $\begin{array}{c}- \\
0.002\end{array}$ & 995. & 0. & 294.5 & -1.0 & 1.00 \\
\hline 2.15 & 183. & $\begin{array}{c}- \\
0.002 \\
\end{array}$ & 1052. & 0. & 294.6 & -1.0 & 1.00 \\
\hline 2.15 & 183. & $\begin{array}{c}- \\
0.002\end{array}$ & 1104. & 0. & 294.6 & -1.0 & 1.00 \\
\hline 2.15 & 183. & $\begin{array}{c}- \\
0.002\end{array}$ & 1156. & 0. & 294.6 & $\begin{array}{l}-1.1 \\
\end{array}$ & 1.00 \\
\hline 2.15 & 183. & $\begin{array}{c}- \\
0.002\end{array}$ & 1216. & 0. & 294.7 & -1.1 & 1.00 \\
\hline 2.15 & 183. & $\begin{array}{c}- \\
0.002\end{array}$ & 1268. & 0. & 294.7 & -1.1 & 1.00 \\
\hline 2.15 & 183. & $\begin{array}{c}- \\
0.002 \\
\end{array}$ & 1322. & 0. & 294.7 & -1.2 & 1.00 \\
\hline 2.15 & 183. & $\begin{array}{c}- \\
0.002\end{array}$ & 1377. & 0. & 294.8 & -1.2 & 1.00 \\
\hline 2.15 & 183. & $\begin{array}{c}- \\
0.002\end{array}$ & 1429. & 0. & 294.8 & -1.2 & 1.00 \\
\hline 2.62 & 222. & 0.024 & 1620. & 0. & 294.9 & -1.3 & 1.00 \\
\hline 2.62 & 222. & 0.024 & 1672. & 0. & 294.9 & -1.4 & 1.00 \\
\hline 2.62 & 222. & 0.024 & 1726. & 0. & 294.9 & -1.4 & 1.00 \\
\hline 2.62 & 222. & 0.024 & 1786. & 0. & 295.0 & -1.4 & 1.00 \\
\hline 2.62 & 222. & 0.024 & 1838. & 0. & 295.0 & -1.4 & 1.00 \\
\hline 2.62 & 222. & 0.024 & 1895. & 0. & 295.0 & -1.5 & 1.00 \\
\hline 2.62 & 222. & 0.024 & 1948. & 0. & 295.0 & -1.5 & 1.00 \\
\hline 2.62 & 222. & 0.024 & 2017. & 0. & 295.1 & -1.5 & 1.00 \\
\hline 2.62 & 222. & 0.024 & 2072. & 0. & 295.1 & -1.5 & 1.00 \\
\hline 2.62 & 222. & 0.024 & 2124. & 0. & 295.1 & -1.5 & 1.00 \\
\hline 2.62 & 222. & 0.024 & 2177. & 0. & 295.1 & -1.6 & 1.00 \\
\hline 2.62 & 222. & 0.024 & 2231. & 0. & 295.1 & -1.6 & 1.00 \\
\hline 2.62 & 222. & 0.024 & 2283. & 0. & 295.2 & -1.6 & 1.00 \\
\hline 3.22 & 274. & $\begin{array}{c}- \\
0.041\end{array}$ & 6047. & 0. & 295.8 & -2.2 & 1.00 \\
\hline 3.22 & 274. & $\begin{array}{c}- \\
0.041 \\
\end{array}$ & 6098. & 0. & 295.7 & -2.2 & 1.00 \\
\hline 3.22 & 274. & $\begin{array}{c}- \\
0.041 \\
\end{array}$ & 6150. & 0. & 295.7 & -2.2 & 1.00 \\
\hline 3.22 & 274. & $\begin{array}{c}-\overline{-} \\
0.041\end{array}$ & 6206. & 0. & 295.8 & -2.2 & 1.00 \\
\hline 3.22 & 274. & $\begin{array}{c}- \\
0.041\end{array}$ & 6259. & 0. & 295.7 & -2.2 & 1.00 \\
\hline 3.22 & 274. & $\begin{array}{c}- \\
0.041\end{array}$ & 6312. & 0. & 295.8 & -2.2 & 1.00 \\
\hline 3.22 & 274. & $\begin{array}{c}- \\
0.041 \\
\end{array}$ & 6367. & 0. & 295.8 & -2.2 & 1.00 \\
\hline 3.22 & 274. & $\begin{array}{c}- \\
0.041\end{array}$ & 6420. & 0. & 295.8 & -2.2 & 1.00 \\
\hline 3.22 & 274. & $\begin{array}{c}- \\
0.041\end{array}$ & 6471. & 0. & 295.8 & -2.2 & 1.00 \\
\hline 3.22 & 274. & $\begin{array}{c}- \\
0.041 \\
\end{array}$ & 6522. & 0. & 295.8 & -2.2 & 1.00 \\
\hline 3.22 & 274. & $\begin{array}{c}- \\
0.041\end{array}$ & 6575. & 0. & 295.8 & -2.2 & 1.00 \\
\hline 3.22 & 274. & $\begin{array}{c}- \\
0.041 \\
\end{array}$ & 6628. & 0. & 295.8 & -2.2 & 1.00 \\
\hline 3.22 & 274. & $\begin{array}{c}- \\
0.041 \\
\end{array}$ & 6686. & 0. & 295.8 & -2.2 & 1.00 \\
\hline 4.75 & 403. & 0.042 & 9446. & 0. & 295.9 & $\begin{array}{l}-2.4 \\
\end{array}$ & 1.00 \\
\hline 4.75 & 403. & 0.042 & 9497. & 0. & 295.9 & -2.3 & 1.00 \\
\hline 4.75 & 403. & 0.042 & 9553. & 0. & 295.9 & -2.4 & 1.00 \\
\hline 4.75 & 403. & 0.042 & 9603. & 0. & 295.9 & -2.4 & 1.00 \\
\hline 4.75 & 403. & 0.042 & 9655. & 0. & 295.9 & -2.4 & 1.00 \\
\hline 4.75 & 403. & 0.042 & 9707. & 0. & 295.9 & -2.4 & 1.00 \\
\hline 4.75 & 403. & 0.042 & 9764. & 0. & 295.9 & -2.4 & 1.00 \\
\hline 4.75 & 403. & 0.042 & 9818. & 0. & 295.9 & -2.4 & 1.00 \\
\hline 4.75 & 403. & 0.042 & 9872. & 0. & 295.9 & -2.4 & 1.00 \\
\hline 4.75 & 403. & 0.042 & 9924. & 0. & 295.9 & $\begin{array}{l}-2.4 \\
\end{array}$ & 1.00 \\
\hline 4.75 & 403. & 0.042 & 9981. & 0. & 295.9 & -2.4 & 1.00 \\
\hline 4.75 & 403. & 0.042 & 10034. & 0. & 295.9 & -2.4 & 1.00 \\
\hline 4.75 & 403. & 0.042 & 10087. & 0. & 295.9 & -2.4 & 1.00 \\
\hline
\end{tabular}

\begin{tabular}{|c|c|c|c|c|c|c|c|}
\hline 4.97 & 422. & 0.059 & 10243. & 0. & 295.9 & -2.4 & 1.00 \\
\hline 4.97 & 422. & 0.059 & 10300 . & 0. & 295.9 & -2.4 & 1.00 \\
\hline 4.97 & 422. & 0.059 & 10355. & 0. & 296.0 & -2.4 & 1.00 \\
\hline 4.97 & 422. & 0.059 & 10409. & 0. & 295.9 & -2.4 & 1.00 \\
\hline 4.97 & 422. & 0.059 & 10463. & 0. & 296.0 & -2.4 & 1.00 \\
\hline 4.97 & 422. & 0.059 & 10518. & 0. & 296.0 & -2.4 & 1.00 \\
\hline 4.97 & 422. & 0.059 & 10574. & 0. & 296.0 & -2.4 & 1.00 \\
\hline 4.97 & 422. & 0.059 & 10625. & 0. & 296.0 & -2.4 & 1.00 \\
\hline 4.97 & 422. & 0.059 & 10682. & 0. & 296.0 & -2.4 & 1.00 \\
\hline 4.97 & 422. & 0.059 & 10734. & 0. & 296.0 & -2.4 & 1.00 \\
\hline 4.97 & 422. & 0.059 & 10842. & 0. & 296.0 & -2.4 & 1.00 \\
\hline 4.97 & 422. & 0.059 & 10893. & 0. & 296.0 & -2.4 & 1.00 \\
\hline 4.97 & 422. & 0.059 & 10945. & 0. & 296.0 & -2.4 & 1.00 \\
\hline 5.25 & 445. & 0.045 & 11099. & 0. & 296.0 & -2.4 & 1.00 \\
\hline 5.25 & 445. & 0.045 & 11153. & 0. & 296.0 & -2.4 & 1.00 \\
\hline 5.25 & 445. & 0.045 & 11209. & 0. & 296.0 & -2.4 & 1.00 \\
\hline 5.25 & 445. & 0.045 & 11263. & 0. & 296.0 & -2.4 & 1.00 \\
\hline 5.25 & 445. & 0.045 & 11319. & 0. & 296.0 & -2.4 & 1.00 \\
\hline 5.25 & 445. & 0.045 & 11378. & 0. & 296.0 & -2.4 & 1.00 \\
\hline 5.25 & 445. & 0.045 & 11430. & 0. & 296.0 & -2.5 & 1.00 \\
\hline 5.25 & 445. & 0.045 & 11484. & 0. & 296.0 & -2.5 & 1.00 \\
\hline 5.25 & 445. & 0.045 & 11540. & 0. & 296.0 & -2.4 & 1.00 \\
\hline 5.25 & 445. & 0.045 & 11596. & 0. & 296.0 & -2.5 & 1.00 \\
\hline 5.25 & 445. & 0.045 & 11648. & 0. & 296.0 & -2.4 & 1.00 \\
\hline 5.25 & 445. & 0.045 & 11704. & 0. & 296.0 & -2.5 & 1.00 \\
\hline 5.25 & 445. & 0.045 & 11763. & 0. & 296.0 & -2.5 & 1.00 \\
\hline 1.34 & 113. & 0.363 & 78324. & 0. & 295.7 & -2.2 & 1.00 \\
\hline 1.34 & 113. & 0.363 & 78374 . & 0. & 295.8 & -2.2 & 1.00 \\
\hline 1.34 & 113. & 0.363 & 78427. & 0. & 295.7 & -2.2 & 1.00 \\
\hline 1.34 & 113. & 0.363 & 78479. & 0. & 295.7 & -2.2 & 1.00 \\
\hline 1.34 & 113. & 0.363 & 78531. & 0. & 295.8 & -2.2 & 1.00 \\
\hline 1.34 & 113. & 0.363 & 78588. & 0. & 295.7 & -2.2 & 1.00 \\
\hline 1.34 & 113. & 0.363 & 78641. & 0. & 295.8 & -2.2 & 1.00 \\
\hline 1.34 & 113. & 0.363 & 78695. & 0. & 295.7 & -2.2 & 1.00 \\
\hline 1.34 & 113. & 0.363 & 78748. & 0. & 295.8 & -2.2 & 1.00 \\
\hline 1.34 & 113. & 0.363 & 78800 . & 0. & 295.8 & -2.2 & 1.00 \\
\hline 1.34 & 113. & 0.363 & 78853. & 0. & 295.7 & -2.2 & 1.00 \\
\hline 1.34 & 113. & 0.363 & 78906. & 0. & 295.8 & -2.2 & 1.00 \\
\hline 1.34 & 113. & 0.363 & 78959. & 0. & 295.8 & -2.2 & 1.00 \\
\hline 0.79 & 67. & 0.316 & 82641. & 0. & 295.8 & -2.2 & 1.00 \\
\hline 0.79 & 67. & 0.316 & 82691. & 0. & 295.8 & -2.2 & 1.00 \\
\hline 0.79 & 67. & 0.316 & 82741. & 0. & 295.8 & -2.2 & 1.00 \\
\hline 0.79 & 67. & 0.316 & 82795. & 0. & 295.8 & -2.2 & 1.00 \\
\hline 0.79 & 67. & 0.316 & 82850 . & 0. & 295.8 & -2.2 & 1.00 \\
\hline 0.79 & 67. & 0.316 & 82904. & 0. & 295.8 & -2.2 & 1.00 \\
\hline 0.79 & 67. & 0.316 & 82961. & 0. & 295.8 & -2.2 & 1.00 \\
\hline 0.79 & 67. & 0.316 & 83012. & 0. & 295.8 & -2.2 & 1.00 \\
\hline 0.79 & 67. & 0.316 & 83068. & 0. & 295.8 & -2.2 & 1.00 \\
\hline 0.79 & 67. & 0.316 & 83125. & 0. & 295.8 & -2.2 & 1.00 \\
\hline 0.79 & 67. & 0.316 & 83193. & 0. & 295.8 & -2.2 & 1.00 \\
\hline 0.79 & 67. & 0.316 & 83247. & 0. & 295.8 & -2.2 & 1.00 \\
\hline 0.79 & 67. & 0.316 & 83307. & 0. & 295.8 & -2.2 & 1.00 \\
\hline 0.90 & 76. & 0.299 & 86829. & 0. & 295.8 & -2.2 & 1.00 \\
\hline 0.90 & 76. & 0.299 & 86883. & 0. & 295.7 & -2.2 & 1.00 \\
\hline 0.90 & 76. & 0.299 & 86940. & 0. & 295.7 & -2.2 & 1.00 \\
\hline 0.90 & 76. & 0.299 & 86991. & 0. & 295.7 & -2.2 & 1.00 \\
\hline 0.90 & 76. & 0.299 & 87042 . & 0. & 295.7 & -2.2 & 1.00 \\
\hline 0.90 & 76. & 0.299 & 87094. & 0. & 295.7 & -2.2 & 1.00 \\
\hline 0.90 & 76. & 0.299 & 87146. & 0. & 295.7 & -2.2 & 1.00 \\
\hline 0.90 & 76. & 0.299 & 87199. & 0. & 295.7 & -2.2 & 1.00 \\
\hline 0.90 & 76. & 0.299 & 87252. & 0. & 295.7 & -2.2 & 1.00 \\
\hline 0.90 & 76. & 0.299 & 87308. & 0. & 295.7 & -2.2 & 1.00 \\
\hline 0.90 & 76. & 0.299 & 87361. & 0. & 295.8 & -2.2 & 1.00 \\
\hline 0.90 & 76. & 0.299 & 87411. & 0. & 295.7 & -2.2 & 1.00 \\
\hline 0.90 & 76. & 0.299 & 87465. & 0. & 295.7 & -2.2 & 1.00 \\
\hline 1.38 & 117. & 0.281 & 87638. & 0. & 295.8 & -2.2 & 1.00 \\
\hline 1.38 & 117. & 0.281 & 87693. & 0. & 295.8 & -2.2 & 1.00 \\
\hline 1.38 & 117. & 0.281 & 87760 . & 0. & 295.8 & -2.2 & 1.00 \\
\hline 1.38 & 117. & 0.281 & 87817. & 0. & 295.8 & -2.2 & 1.00 \\
\hline 1.38 & 117. & 0.281 & 87869. & 0. & 295.8 & -2.2 & 1.00 \\
\hline 1.38 & 117. & 0.281 & 87921. & 0. & 295.8 & -2.2 & 1.00 \\
\hline 1.38 & 117. & 0.281 & 87975. & 0. & 295.8 & -2.2 & 1.00 \\
\hline 1.38 & 117. & 0.281 & 88028. & 0. & 295.8 & -2.2 & 1.00 \\
\hline 1.38 & 117. & 0.281 & 88084. & 0. & 295.8 & -2.2 & 1.00 \\
\hline 1.38 & 117. & 0.281 & 88139. & 0. & 295.8 & -2.2 & 1.00 \\
\hline 1.38 & 117. & 0.281 & 88195. & 0. & 295.8 & -2.2 & 1.00 \\
\hline 1.38 & 117. & 0.281 & 88247. & 0. & 295.8 & -2.2 & 1.00 \\
\hline 1.38 & 117. & 0.281 & 88303. & 0. & 295.8 & -2.2 & 1.00 \\
\hline 1.27 & 108. & 0.265 & 93296. & 0. & 295.9 & -2.3 & 1.00 \\
\hline 1.27 & 108. & 0.265 & 93361. & 0. & 295.9 & -2.3 & 1.00 \\
\hline
\end{tabular}


Table H.2.2 Diesel contamination on oxidized copper surface for $\mathrm{Re}=1900$ and $x_{\mathrm{b}}=0.2 \%$ (file:trv15con1.tb2)

\begin{tabular}{|c|c|c|c|c|c|c|c|}
\hline $\begin{array}{c}l_{\mathrm{e}} \\
(\mu \mathrm{m})\end{array}$ & $\begin{array}{c}\Gamma \\
\left(\mathrm{kg} / \mathrm{m}^{2}\right) \\
\mathrm{X} 10^{5} \\
\end{array}$ & $\begin{array}{c}x_{\mathrm{b}} \\
(\%) \\
\end{array}$ & $\begin{array}{l}\text { Exposure } \\
\text { Time (s) }\end{array}$ & $\mathrm{Re}$ & 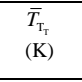 & $\begin{array}{c}T_{\mathrm{b}}-\bar{T}_{\mathrm{T}_{\mathrm{T}}} \\
(\mathrm{K})\end{array}$ & $\frac{F_{T_{b}}}{F_{T_{T}}}$ \\
\hline 0.20 & 17. & 0.285 & 1008. & 1643. & 295.5 & -1.8 & 1.00 \\
\hline 0.20 & 17. & 0.285 & 1065. & 1587. & 295.5 & -1.8 & 1.00 \\
\hline 0.20 & 17. & 0.285 & 1135. & 1633. & 295.4 & -1.8 & 1.00 \\
\hline 0.20 & 17. & 0.285 & 1179. & 1597. & 295.4 & -1.8 & 1.00 \\
\hline 0.20 & 17. & 0.285 & 1258. & 1613. & 295.4 & -1.8 & 1.00 \\
\hline 0.20 & 17. & 0.285 & 1321. & 1623. & 295.4 & -1.7 & 1.00 \\
\hline 0.20 & 17. & 0.285 & 1392. & 1529. & 295.3 & -1.7 & 1.00 \\
\hline 0.20 & 17. & 0.285 & 1492. & 1571. & 295.3 & -1.7 & 1.00 \\
\hline 0.20 & 17. & 0.285 & 1536. & 1591. & 295.3 & -1.6 & 1.00 \\
\hline 0.20 & 17. & 0.285 & 1574. & 1597. & 295.2 & -1.6 & 1.00 \\
\hline 0.20 & 17. & 0.285 & 1617. & 1521. & 295.2 & -1.6 & 1.00 \\
\hline 0.20 & 17. & 0.285 & 1661. & 1542. & 295.2 & -1.6 & 1.00 \\
\hline 0.20 & 17. & 0.285 & 1705. & 1599. & 295.2 & -1.5 & 1.00 \\
\hline 0.27 & 23. & 0.198 & 61523. & 1739. & 293.5 & 0.1 & 1.00 \\
\hline 0.27 & 23. & 0.198 & 61568. & 1800. & 293.5 & 0.1 & 1.00 \\
\hline 0.27 & 23. & 0.198 & 61614. & 1778. & 293.5 & 0.2 & 1.00 \\
\hline 0.27 & 23. & 0.198 & 61656 . & 1787. & 293.4 & 0.2 & 1.00 \\
\hline 0.27 & 23. & 0.198 & 61697. & 1788. & 293.4 & 0.2 & 1.00 \\
\hline 0.27 & 23. & 0.198 & 61743. & 1802. & 293.4 & 0.2 & 1.00 \\
\hline 0.27 & 23. & 0.198 & 61782 . & 1712. & 293.4 & 0.2 & 1.00 \\
\hline 0.27 & 23. & 0.198 & 61823. & 1778. & 293.4 & 0.3 & 1.00 \\
\hline 0.27 & 23. & 0.198 & 61865. & 1750. & 293.4 & 0.3 & 1.00 \\
\hline 0.27 & 23. & 0.198 & 61905. & 1697. & 293.4 & 0.3 & 1.00 \\
\hline 0.27 & 23. & 0.198 & 61946 . & 1776. & 293.3 & 0.3 & 1.00 \\
\hline 0.27 & 23. & 0.198 & 61987. & 1759. & 293.3 & 0.3 & 1.00 \\
\hline 0.27 & 23. & 0.198 & 62026 . & 1794. & 293.3 & 0.3 & 1.00 \\
\hline 0.65 & 55. & 0.179 & 62198. & 1706. & 293.3 & 0.4 & 1.00 \\
\hline 0.65 & 55. & 0.179 & 62241. & 1737. & 293.3 & 0.4 & 1.00 \\
\hline 0.65 & 55. & 0.179 & 62282 . & 1781. & 293.2 & 0.4 & 1.00 \\
\hline 0.65 & 55. & 0.179 & 62324 . & 1746. & 293.2 & 0.4 & 1.00 \\
\hline 0.65 & 55. & 0.179 & 62367 . & 1705. & 293.2 & 0.4 & 1.00 \\
\hline 0.65 & 55. & 0.179 & 62409. & 1785. & 293.2 & 0.4 & 1.00 \\
\hline 0.65 & 55. & 0.179 & 62454. & 1743. & 293.2 & 0.4 & 1.00 \\
\hline 0.65 & 55. & 0.179 & 62498 . & 1756. & 293.2 & 0.4 & 1.00 \\
\hline 0.65 & 55. & 0.179 & 62539. & 1696. & 293.2 & 0.4 & 1.00 \\
\hline 0.65 & 55. & 0.179 & 62581. & 1763. & 293.2 & 0.4 & 1.00 \\
\hline 0.65 & 55. & 0.179 & 62622 . & 1765. & 293.2 & 0.4 & 1.00 \\
\hline 0.65 & 55. & 0.179 & 62664 . & 1776 . & 293.2 & 0.4 & 1.00 \\
\hline 0.65 & 55. & 0.179 & 62707. & 1725. & 293.2 & 0.4 & 1.00 \\
\hline 1.68 & 143. & 0.152 & 66387. & 1801. & 293.7 & 0.0 & 1.00 \\
\hline 1.68 & 143. & 0.152 & 66427. & 1783. & 293.7 & 0.0 & 1.00 \\
\hline 1.68 & 143. & 0.152 & 66469. & 1746. & 293.6 & 0.0 & 1.00 \\
\hline 1.68 & 143. & 0.152 & 66509. & 1824. & 293.6 & 0.0 & 1.00 \\
\hline 1.68 & 143. & 0.152 & 66551. & 1810. & 293.6 & 0.1 & 1.00 \\
\hline 1.68 & 143. & 0.152 & 66592 . & 1810. & 293.6 & 0.1 & 1.00 \\
\hline 1.68 & 143. & 0.152 & 66632 . & 1784. & 293.5 & 0.1 & 1.00 \\
\hline 1.68 & 143. & 0.152 & 66709. & 1682. & 293.5 & 0.1 & 1.00 \\
\hline 1.68 & 143. & 0.152 & 66749. & 1722. & 293.5 & 0.1 & 1.00 \\
\hline 1.68 & 143. & 0.152 & 66788. & 1779. & 293.5 & 0.2 & 1.00 \\
\hline 1.68 & 143. & 0.152 & 66829. & 1706. & 293.4 & 0.2 & 1.00 \\
\hline 1.68 & 143. & 0.152 & 66869 . & 1794. & 293.4 & 0.2 & 1.00 \\
\hline 1.68 & 143. & 0.152 & 66910. & 1748. & 293.4 & 0.2 & 1.00 \\
\hline 0.43 & 36. & 0.250 & 69847. & 1776 . & 294.0 & -0.3 & 1.00 \\
\hline 0.43 & 36. & 0.250 & 69884 . & 1736. & 294.0 & -0.3 & 1.00 \\
\hline 0.43 & 36. & 0.250 & 69926 . & 1785. & 294.0 & -0.3 & 1.00 \\
\hline 0.43 & 36. & 0.250 & 69967. & 1789. & 294.0 & -0.3 & 1.00 \\
\hline 0.43 & 36. & 0.250 & 70009. & 1795. & 294.0 & -0.4 & 1.00 \\
\hline 0.43 & 36. & 0.250 & 70049. & 1714. & 294.0 & -0.3 & 1.00 \\
\hline 0.43 & 36. & 0.250 & 70091. & 1827. & 294.0 & -0.3 & 1.00 \\
\hline 0.43 & 36. & 0.250 & 70131. & 1768. & 294.0 & -0.4 & 1.00 \\
\hline 0.43 & 36. & 0.250 & 70173. & 1799. & 294.0 & -0.3 & 1.00 \\
\hline 0.43 & 36. & 0.250 & 70212 . & 1727. & 294.0 & -0.3 & 1.00 \\
\hline 0.43 & 36. & 0.250 & 70255. & 1754. & 294.0 & -0.3 & 1.00 \\
\hline 0.43 & 36. & 0.250 & 70295. & 1770. & 294.0 & -0.3 & 1.00 \\
\hline 0.43 & 36. & 0.250 & 70333. & 1774. & 294.0 & -0.3 & 1.00 \\
\hline 0.24 & 21. & 0.207 & 70481. & 1785 . & 294.0 & -0.3 & 1.00 \\
\hline 0.24 & 21. & 0.207 & 70523. & 1783. & 294.0 & -0.3 & 1.00 \\
\hline 0.24 & 21. & 0.207 & 70562 . & 1749. & 293.9 & -0.3 & 1.00 \\
\hline 0.24 & 21. & 0.207 & 70603. & 1732. & 293.9 & -0.3 & 1.00 \\
\hline 0.24 & 21. & 0.207 & 70645 . & 1792. & 293.9 & -0.3 & 1.00 \\
\hline 0.24 & 21. & 0.207 & 70685 . & 1825. & 293.9 & -0.3 & 1.00 \\
\hline 0.24 & 21. & 0.207 & 70726 . & 1843. & 293.9 & -0.3 & 1.00 \\
\hline 0.24 & 21. & 0.207 & 70767 . & 1785. & 293.9 & -0.3 & 1.00 \\
\hline 0.24 & 21. & 0.207 & 70807. & 1772 . & 293.9 & -0.2 & 1.00 \\
\hline 0.24 & 21. & 0.207 & 70846 . & 1707. & 293.9 & -0.2 & 1.00 \\
\hline 0.24 & 21. & 0.207 & 70886 . & 1728. & 293.8 & -0.2 & 1.00 \\
\hline 0.24 & 21. & 0.207 & 70928 . & 1706. & 293.9 & -0.2 & 1.00 \\
\hline 0.24 & 21. & 0.207 & 70968 . & 1734. & 293.8 & -0.2 & 1.00 \\
\hline
\end{tabular}

\begin{tabular}{|c|c|c|c|c|c|c|c|}
\hline 0.06 & 5. & 0.202 & 71125. & 1826. & 293.8 & -0.1 & 1.00 \\
\hline 0.06 & 5 . & 0.202 & 71164. & 1779. & 293.7 & -0.1 & 1.00 \\
\hline 0.06 & 5. & 0.202 & 71204 & 1803. & 293.7 & -0.1 & 1.00 \\
\hline 0.06 & 5. & 0.202 & 71251. & 1747. & 293.7 & -0.1 & 1.00 \\
\hline 0.06 & 5. & 0.202 & 71295. & 1755. & 293.7 & -0.1 & 1.00 \\
\hline 0.06 & 5. & 0.202 & 71336. & 1754. & 293.6 & 0.0 & 1.00 \\
\hline 0.06 & 5. & 0.202 & 71379. & 1723. & 293.6 & 0.0 & 1.00 \\
\hline 0.06 & 5. & 0.202 & 71420 . & 1732 . & 293.6 & 0.0 & 1.00 \\
\hline 0.06 & 5. & 0.202 & 71461. & 1746. & 293.6 & 0.0 & 1.00 \\
\hline 0.06 & 5. & 0.202 & 71503. & 1797. & 293.6 & 0.1 & 1.00 \\
\hline 0.06 & 5. & 0.202 & 71543. & 1784. & 293.6 & 0.1 & 1.00 \\
\hline 0.06 & 5. & 0.202 & 71583. & 1778 . & 293.5 & 0.1 & 1.00 \\
\hline 0.06 & 5. & 0.202 & 71624 . & 1806. & 293.5 & 0.1 & 1.00 \\
\hline-0.09 & -8. & 0.204 & 80588 . & 1803. & 293.9 & -0.3 & 1.00 \\
\hline-0.09 & -8. & 0.204 & 80631. & 1813. & 293.9 & -0.3 & 1.00 \\
\hline-0.09 & -8 & 0.204 & 80674 . & 1820. & 293.9 & -0.3 & 1.00 \\
\hline-0.09 & -8. & 0.204 & 80717. & 1743. & 293.9 & -0.3 & 1.00 \\
\hline-0.09 & -8. & 0.204 & 80759. & 1819. & 293.9 & -0.2 & 1.00 \\
\hline-0.09 & -8. & 0.204 & 80803. & 1783. & 293.9 & -0.2 & 1.00 \\
\hline-0.09 & -8. & 0.204 & 80848 & 1784. & 293.8 & -0.2 & 1.00 \\
\hline-0.09 & -8 & 0.204 & 80892 & 1723. & 293.8 & -0.2 & 1.00 \\
\hline-0.09 & -8. & 0.204 & 80933. & 1845. & 293.8 & -0.2 & 1.00 \\
\hline-0.09 & -8. & 0.204 & 80977. & 1749. & 293.8 & -0.1 & 1.00 \\
\hline-0.09 & -8. & 0.204 & 81018. & 1707. & 293.8 & -0.1 & 1.00 \\
\hline-0.09 & -8. & 0.204 & 81061. & 1803. & 293.7 & -0.1 & 1.00 \\
\hline-0.09 & -8. & 0.204 & 81106. & 1755. & 293.7 & -0.1 & 1.00 \\
\hline-0.12 & -10. & 0.201 & 84789. & 1751. & 294.0 & -0.3 & 1.00 \\
\hline-0.12 & -10. & 0.201 & 84836 . & 1730. & 294.0 & -0.3 & 1.00 \\
\hline-0.12 & -10. & 0.201 & 84888 . & 1726. & 294.0 & -0.3 & 1.00 \\
\hline-0.12 & -10. & 0.201 & 84954. & 1805. & 294.0 & -0.3 & 1.00 \\
\hline-0.12 & -10. & 0.201 & 85002 . & 1797. & 294.0 & -0.3 & 1.00 \\
\hline-0.12 & -10. & 0.201 & 85046 . & 1695. & 294.0 & -0.3 & 1.00 \\
\hline-0.12 & -10. & 0.201 & 85085 . & 1726. & 294.0 & -0.3 & 1.00 \\
\hline-0.12 & -10. & 0.201 & 85122. & 1713. & 294.0 & -0.3 & 1.00 \\
\hline-0.12 & -10. & 0.201 & 85173. & 1834. & 294.0 & -0.3 & 1.00 \\
\hline-0.12 & -10. & 0.201 & 85210 . & 1821. & 294.0 & -0.3 & 1.00 \\
\hline-0.12 & -10. & 0.201 & 85250 . & 1825. & 294.0 & -0.3 & 1.00 \\
\hline-0.12 & -10. & 0.201 & 85289. & 1823. & 293.9 & -0.3 & 1.00 \\
\hline-0.12 & -10. & 0.201 & 85357. & 1820. & 293.9 & -0.3 & 1.00 \\
\hline-0.09 & -8. & 0.187 & 150554. & 1704. & 293.9 & -0.3 & 1.00 \\
\hline-0.09 & -8. & 0.187 & 150599. & 1729. & 293.9 & -0.3 & 1.00 \\
\hline-0.09 & -8. & 0.187 & 150645 . & 1782. & 293.9 & -0.3 & 1.00 \\
\hline-0.09 & -8. & 0.187 & 150688. & 1831. & 293.9 & -0.2 & 1.00 \\
\hline-0.09 & -8. & 0.187 & 150735. & 1716. & 293.8 & -0.2 & 1.00 \\
\hline-0.09 & -8. & 0.187 & 150775 . & 1792. & 293.8 & -0.2 & 1.00 \\
\hline-0.09 & -8. & 0.187 & 150817. & 1743. & 293.8 & -0.2 & 1.00 \\
\hline-0.09 & -8. & 0.187 & 150855. & 1796. & 293.8 & -0.2 & 1.00 \\
\hline-0.09 & -8. & 0.187 & 150898 . & 1764. & 293.8 & -0.1 & 1.00 \\
\hline-0.09 & -8. & 0.187 & 150937. & 1733. & 293.8 & -0.1 & 1.00 \\
\hline-0.09 & -8. & 0.187 & 150978. & 1721. & 293.7 & -0.1 & 1.00 \\
\hline-0.09 & -8. & 0.187 & 151018. & 1766. & 293.7 & -0.1 & 1.00 \\
\hline-0.09 & -8. & 0.187 & 151060. & 1780. & 293.7 & -0.1 & 1.00 \\
\hline-0.15 & -13. & 0.191 & 151192. & 1726. & 293.6 & 0.0 & 1.00 \\
\hline-0.15 & -13. & 0.191 & 151234. & 1711. & 293.6 & 0.0 & 1.00 \\
\hline-0.15 & -13. & 0.191 & 151277. & 1800. & 293.6 & 0.0 & 1.00 \\
\hline-0.15 & -13. & 0.191 & 151319. & 1796. & 293.6 & 0.0 & 1.00 \\
\hline-0.15 & -13. & 0.191 & 151364. & 1786. & 293.6 & 0.1 & 1.00 \\
\hline-0.15 & -13. & 0.191 & 151406. & 1767. & 293.6 & 0.1 & 1.00 \\
\hline-0.15 & -13. & 0.191 & 151448. & 1784. & 293.5 & 0.1 & 1.00 \\
\hline-0.15 & -13. & 0.191 & 151489. & 1795. & 293.5 & 0.1 & 1.00 \\
\hline-0.15 & -13. & 0.191 & 151529. & 1815. & 293.5 & 0.1 & 1.00 \\
\hline-0.15 & -13. & 0.191 & 151569. & 1691. & 293.4 & 0.2 & 1.00 \\
\hline-0.15 & -13. & 0.191 & 151609. & 1727. & 293.4 & 0.2 & 1.00 \\
\hline-0.15 & -13. & 0.191 & 151650. & 1716. & 293.4 & 0.2 & 1.00 \\
\hline-0.15 & -13. & 0.191 & 151691. & 1723. & 293.4 & 0.2 & 1.00 \\
\hline-0.17 & -14. & 0.194 & 154873. & 1797. & 294.0 & -0.3 & 1.00 \\
\hline-0.17 & -14. & 0.194 & 154913. & 1770. & 294.0 & -0.3 & 1.00 \\
\hline-0.17 & -14. & 0.194 & 154958. & 1774. & 294.0 & -0.3 & 1.00 \\
\hline-0.17 & -14. & 0.194 & 155004. & 1739. & 294.0 & -0.3 & 1.00 \\
\hline-0.17 & -14. & 0.194 & 155046. & 1791. & 294.0 & -0.3 & 1.00 \\
\hline-0.17 & -14. & 0.194 & 155087. & 1816. & 294.0 & -0.3 & 1.00 \\
\hline-0.17 & -14. & 0.194 & 155128. & 1847. & 294.0 & -0.3 & 1.00 \\
\hline-0.17 & -14. & 0.194 & 155168. & 1811. & 294.0 & -0.3 & 1.00 \\
\hline-0.17 & -14. & 0.194 & 155208. & 1811. & 294.0 & -0.3 & 1.00 \\
\hline-0.17 & -14. & 0.194 & 155248. & 1718. & 294.0 & -0.3 & 1.00 \\
\hline-0.17 & -14. & 0.194 & 155292 . & 1815. & 294.0 & -0.3 & 1.00 \\
\hline-0.17 & -14. & 0.194 & 155333. & 1753. & 294.0 & -0.3 & 1.00 \\
\hline-0.17 & -14. & 0.194 & 155377. & 1738. & 294.0 & -0.3 & 1.00 \\
\hline-0.23 & -20 . & 0.194 & 158222. & 1712 . & 293.3 & 0.3 & 1.00 \\
\hline-0.23 & -20. & 0.194 & 158266 . & 1780. & 293.3 & 0.3 & 1.00 \\
\hline-0.23 & -20 . & 0.194 & 158309. & 1721. & 293.3 & 0.3 & 1.00 \\
\hline
\end{tabular}


Table H.2.3 Diesel contamination on oxidized copper surface for $\operatorname{Re}=3200$ and $x_{\mathrm{b}}=0.2 \%$ (file:trv3con1.tb2)

\begin{tabular}{|c|c|c|c|c|c|c|c|}
\hline $\begin{array}{c}l_{\mathrm{e}} \\
(\mu \mathrm{m})\end{array}$ & $\begin{array}{c}\Gamma \\
\left(\mathrm{kg} / \mathrm{m}^{2}\right) \\
\mathrm{X} 10^{5}\end{array}$ & $\begin{array}{l}x_{\mathrm{b}} \\
(\%)\end{array}$ & $\begin{array}{c}\text { Exposure } \\
\text { Time (s) }\end{array}$ & Re & $\begin{array}{l}\bar{T}_{\mathrm{T}_{\mathrm{T}}} \\
(\mathrm{K})\end{array}$ & $\begin{array}{c}T_{\mathrm{b}}-\bar{T}_{\mathrm{T}_{\mathrm{T}}} \\
(\mathrm{K})\end{array}$ & $\frac{F_{T_{b}}}{F_{T_{T}}}$ \\
\hline-0.36 & -30. & 0.309 & 1521. & 3405. & 296.1 & -2.5 & 1.00 \\
\hline-0.36 & -30. & 0.309 & 1563. & 3305. & 296.2 & -2.5 & 1.00 \\
\hline-0.36 & -30. & 0.309 & 1607. & 3389. & 296.2 & -2.5 & 1.00 \\
\hline-0.36 & -30. & 0.309 & 1706. & 3349. & 296.2 & -2.5 & 1.00 \\
\hline-0.36 & -30 & 0.309 & 1749. & 3300. & 296.2 & -2.5 & 1.00 \\
\hline-0.36 & -30. & 0.309 & 1789. & 3228. & 296.2 & -2.5 & 1.00 \\
\hline-0.36 & -30. & 0.309 & 1831. & 3390. & 296.2 & -2.5 & 1.00 \\
\hline-0.36 & -30. & 0.309 & 1873. & 3178. & 296.2 & -2.5 & 1.00 \\
\hline-0.36 & -30. & 0.309 & 1920. & 3405. & 296.2 & -2.5 & 1.00 \\
\hline-0.36 & -30. & 0.309 & 1966. & 3357. & 296.2 & -2.5 & 1.00 \\
\hline-0.36 & -30. & 0.309 & 2009. & 3390. & 296.2 & -2.5 & 1.00 \\
\hline-0.36 & -30. & 0.309 & 2051. & 3372. & 296.2 & -2.5 & 1.00 \\
\hline-0.36 & -30. & 0.309 & 2092. & 3283. & 296.2 & -2.5 & 1.00 \\
\hline-0.47 & -40. & 0.296 & 2224. & 3216. & 296.2 & -2.5 & 1.00 \\
\hline-0.47 & -40. & 0.296 & 2267. & 3301. & 296.2 & -2.5 & 1.00 \\
\hline-0.47 & -40. & 0.296 & 2310. & 3389. & 296.2 & -2.5 & 1.00 \\
\hline-0.47 & -40. & 0.296 & 2354. & 3356. & 296.2 & -2.5 & 1.00 \\
\hline-0.47 & -40. & 0.296 & 2401. & 3234. & 296.1 & -2.5 & 1.00 \\
\hline-0.47 & -40. & 0.296 & 2442. & 3273. & 296.1 & -2.5 & 1.00 \\
\hline-0.47 & -40. & 0.296 & 2482. & 3378. & 296.1 & -2.5 & 1.00 \\
\hline-0.47 & -40. & 0.296 & 2524. & 3327. & 296.1 & -2.5 & 1.00 \\
\hline-0.47 & -40. & 0.296 & 2569. & 3310. & 296.1 & -2.5 & 1.00 \\
\hline-0.47 & -40. & 0.296 & 2775. & 3244. & 296.1 & -2.4 & 1.00 \\
\hline-0.47 & -40. & 0.296 & 2819. & 3241. & 296.0 & -2.4 & 1.00 \\
\hline-0.47 & -40. & 0.296 & 2862. & 3337. & 296.0 & -2.4 & 1.00 \\
\hline-0.47 & -40. & 0.296 & 2903. & 3194. & 296.0 & -2.4 & 1.00 \\
\hline-0.47 & -40. & 0.268 & 5441. & 3245. & 294.4 & -0.8 & 1.00 \\
\hline-0.47 & -40. & 0.268 & 5486. & 3227. & 294.4 & -0.8 & 1.00 \\
\hline-0.47 & -40. & 0.268 & 5531. & 3253. & 294.4 & -0.7 & 1.00 \\
\hline-0.47 & -40. & 0.268 & 5572. & 3276. & 294.3 & -0.7 & 1.00 \\
\hline-0.47 & -40. & 0.268 & 5619. & 3164. & 294.3 & -0.6 & 1.00 \\
\hline-0.47 & -40. & 0.268 & 5660. & 3209. & 294.3 & -0.6 & 1.00 \\
\hline-0.47 & -40. & 0.268 & 5702. & 3113. & 294.2 & -0.6 & 1.00 \\
\hline-0.47 & -40. & 0.268 & 5744. & 3195. & 294.2 & -0.6 & 1.00 \\
\hline-0.47 & -40 . & 0.268 & 5783. & 3110. & 294.2 & -0.5 & 1.00 \\
\hline-0.47 & -40. & 0.268 & 5824. & 3083. & 294.1 & -0.5 & 1.00 \\
\hline-0.47 & -40. & 0.268 & 5866. & 3242. & 294.1 & -0.4 & 1.00 \\
\hline-0.47 & -40. & 0.268 & 5909. & 3209. & 294.1 & -0.4 & 1.00 \\
\hline-0.47 & -40. & 0.268 & 5957. & 3083. & 294.0 & -0.4 & 1.00 \\
\hline-0.32 & -27 & 0.256 & 8736. & 3192. & 293.2 & 0.5 & 1.00 \\
\hline-0.32 & -27 & 0.256 & 8776. & 3194. & 293.2 & 0.5 & 1.00 \\
\hline-0.32 & -27. & 0.256 & 8821. & 3211. & 293.2 & 0.4 & 1.00 \\
\hline-0.32 & -27. & 0.256 & 8865. & 3118. & 293.2 & 0.4 & 1.00 \\
\hline-0.32 & -27. & 0.256 & 8906. & 3075. & 293.3 & 0.4 & 1.00 \\
\hline-0.32 & -27. & 0.256 & 8946. & 3168. & 293.3 & 0.4 & 1.00 \\
\hline-0.32 & -27. & 0.256 & 8988. & 3098. & 293.3 & 0.3 & 1.00 \\
\hline-0.32 & -27. & 0.256 & 9028. & 3117. & 293.3 & 0.3 & 1.00 \\
\hline-0.32 & -27. & 0.256 & 9070. & 3132. & 293.3 & 0.3 & 1.00 \\
\hline-0.32 & -27. & 0.256 & 9116. & 3236. & 293.3 & 0.3 & 1.00 \\
\hline-0.32 & -27 & 0.256 & 9162. & 3173. & 293.4 & 0.3 & 1.00 \\
\hline-0.32 & -27 & 0.256 & 9204. & 3083. & 293.4 & 0.3 & 1.00 \\
\hline-0.32 & -27. & 0.256 & 9244. & 3093. & 293.4 & 0.2 & 1.00 \\
\hline-0.28 & -24. & 0.246 & 9383. & 3208. & 293.5 & 0.2 & 1.00 \\
\hline-0.28 & -24. & 0.246 & 9424. & 3107. & 293.5 & 0.2 & 1.00 \\
\hline-0.28 & -24. & 0.246 & 9465. & 3177. & 293.5 & 0.1 & 1.00 \\
\hline-0.28 & -24 & 0.246 & 9506. & 3178. & 293.5 & 0.1 & 1.00 \\
\hline-0.28 & -24 & 0.246 & 9548. & 3219. & 293.6 & 0.1 & 1.00 \\
\hline-0.28 & -24. & 0.246 & 9592. & 3213. & 293.6 & 0.1 & 1.00 \\
\hline-0.28 & -24 & 0.246 & 9635. & 3084. & 293.6 & 0.0 & 1.00 \\
\hline-0.28 & -24 & 0.246 & 9677. & 3266. & 293.6 & 0.0 & 1.00 \\
\hline-0.28 & -24. & 0.246 & 9721. & 3196. & 293.6 & 0.0 & 1.00 \\
\hline-0.28 & -24 & 0.246 & 9765. & 3172. & 293.7 & 0.0 & 1.00 \\
\hline-0.28 & -24 & 0.246 & 9807. & 3121. & 293.7 & -0.1 & 1.00 \\
\hline-0.28 & -24. & 0.246 & 9849. & 3154. & 293.7 & $\begin{array}{l}-0.1 \\
\end{array}$ & 1.00 \\
\hline-0.28 & -24. & 0.246 & 9892. & 3171. & 293.7 & $\begin{array}{l}-0.1 \\
\end{array}$ & 1.00 \\
\hline-0.38 & -32. & 0.240 & 10046. & 3258. & 293.8 & -0.2 & 1.00 \\
\hline-0.38 & -32. & 0.240 & 10087. & 3141. & 293.9 & $\begin{array}{l}-0.2 \\
\end{array}$ & 1.00 \\
\hline-0.38 & -32. & 0.240 & 10132. & 3151. & 293.9 & -0.2 & 1.00 \\
\hline-0.38 & -32 & 0.240 & 10175. & 3216. & 293.9 & -0.3 & 1.00 \\
\hline-0.38 & -32. & 0.240 & 10219. & 3071. & 293.9 & $\begin{array}{l}-0.3 \\
\end{array}$ & 1.00 \\
\hline-0.38 & -32. & 0.240 & 10261. & 3186. & 293.9 & $\begin{array}{l}-0.3 \\
\end{array}$ & 1.00 \\
\hline-0.38 & -32. & 0.240 & 10304. & 3088. & 294.0 & -0.3 & 1.00 \\
\hline-0.38 & -32. & 0.240 & 10347. & 3172. & 294.0 & $\begin{array}{l}-0.3 \\
\end{array}$ & 1.00 \\
\hline-0.38 & -32. & 0.240 & 10389. & 3126. & 294.0 & $\begin{array}{l}-0.3 \\
\end{array}$ & 1.00 \\
\hline-0.38 & -32 & 0.240 & 10431. & 3222. & 294.0 & -0.4 & 1.00 \\
\hline-0.38 & -32. & 0.240 & 10471. & 3143. & 294.0 & $\begin{array}{l}-0.4 \\
\end{array}$ & 1.00 \\
\hline
\end{tabular}

\begin{tabular}{|c|c|c|c|c|c|c|c|}
\hline-0.38 & -32. & 0.240 & 10514. & 3143. & 294.0 & -0.4 & 1.00 \\
\hline-0.38 & -32. & 0.240 & 10556. & 3279. & 294.0 & -0.4 & 1.00 \\
\hline-0.55 & -46. & 0.271 & 13307. & 3069. & 293.3 & 0.4 & 1.00 \\
\hline-0.55 & -46. & 0.271 & 13350. & 3054. & 293.3 & 0.4 & 1.00 \\
\hline-0.55 & -46. & 0.271 & 13403. & 3191. & 293.3 & 0.4 & 1.00 \\
\hline-0.55 & -46. & 0.271 & 13445. & 3197. & 293.3 & 0.4 & 1.00 \\
\hline-0.55 & -46. & 0.271 & 13492. & 3128. & 293.3 & 0.4 & 1.00 \\
\hline-0.55 & -46. & 0.271 & 13541. & 3119. & 293.3 & 0.4 & 1.00 \\
\hline-0.55 & -46. & 0.271 & 13582. & 3097. & 293.3 & 0.4 & 1.00 \\
\hline-0.55 & -46. & 0.271 & 13624. & 3145. & 293.3 & 0.4 & 1.00 \\
\hline-0.55 & -46. & 0.271 & 13665. & 3128. & 293.3 & 0.4 & 1.00 \\
\hline-0.55 & -46. & 0.271 & 13709. & 3106. & 293.3 & 0.4 & 1.00 \\
\hline-0.55 & -46. & 0.271 & 13750. & 3264. & 293.3 & 0.4 & 1.00 \\
\hline-0.55 & -46. & 0.271 & 13807. & 3099. & 293.3 & 0.3 & 1.00 \\
\hline-0.55 & -46. & 0.271 & 13848. & 3183. & 293.3 & 0.4 & 1.00 \\
\hline-0.19 & -17. & 0.214 & 16251. & 3229. & 294.0 & -0.3 & 1.00 \\
\hline-0.19 & -17. & 0.214 & 16296. & 3229. & 294.0 & -0.3 & 1.00 \\
\hline-0.19 & -17. & 0.214 & 16340. & 3229. & 294.0 & -0.3 & 1.00 \\
\hline-0.19 & -17. & 0.214 & 16380. & 3233. & 293.9 & -0.3 & 1.00 \\
\hline-0.19 & -17. & 0.214 & 16428. & 3104. & 293.9 & -0.3 & 1.00 \\
\hline-0.19 & -17. & 0.214 & 16471. & 3209. & 293.9 & -0.3 & 1.00 \\
\hline-0.19 & -17. & 0.214 & 16518. & 3100. & 293.9 & -0.2 & 1.00 \\
\hline-0.19 & -17. & 0.214 & 16559. & 3216. & 293.9 & $\begin{array}{l}-0.3 \\
\end{array}$ & 1.00 \\
\hline-0.19 & -17. & 0.214 & 16607. & 3254. & 293.9 & -0.2 & 1.00 \\
\hline-0.19 & -17. & 0.214 & 16650. & 3041. & 293.9 & -0.2 & 1.00 \\
\hline-0.19 & -17. & 0.214 & 16693. & 3227. & 293.9 & -0.2 & 1.00 \\
\hline-0.19 & -17. & 0.214 & 16735. & 3097. & 293.8 & -0.2 & 1.00 \\
\hline-0.19 & -17. & 0.214 & 16777. & 3266. & 293.8 & -0.2 & 1.00 \\
\hline-0.19 & -16. & 0.227 & 21142. & 3150. & 294.0 & -0.3 & 1.00 \\
\hline-0.19 & -16. & 0.227 & 21186. & 3158. & 294.0 & -0.3 & 1.00 \\
\hline-0.19 & -16. & 0.227 & 21233. & 3203. & 293.9 & -0.3 & 1.00 \\
\hline-0.19 & -16. & 0.227 & 21277. & 3187. & 293.9 & $\begin{array}{l}-0.3 \\
\end{array}$ & 1.00 \\
\hline-0.19 & -16. & 0.227 & 21322. & 3074. & 293.9 & -0.3 & 1.00 \\
\hline-0.19 & -16. & 0.227 & 21366. & 3155. & 293.9 & $\begin{array}{l}-0.3 \\
\end{array}$ & 1.00 \\
\hline-0.19 & -16. & 0.227 & 21413. & 3264. & 293.9 & -0.3 & 1.00 \\
\hline-0.19 & -16. & 0.227 & 21458. & 3205. & 293.9 & -0.2 & 1.00 \\
\hline-0.19 & -16. & 0.227 & 21504. & 3278. & 293.9 & -0.2 & 1.00 \\
\hline-0.19 & -16. & 0.227 & 21548. & 3142. & 293.9 & -0.2 & 1.00 \\
\hline-0.19 & -16. & 0.227 & 21591. & 3180. & 293.8 & -0.2 & 1.00 \\
\hline-0.19 & -16. & 0.227 & 21640. & 3140. & 293.8 & -0.2 & 1.00 \\
\hline-0.19 & -16. & 0.227 & 21684. & 3209. & 293.8 & -0.2 & 1.00 \\
\hline 0.21 & 18. & 0.203 & 21839. & 3237. & 293.8 & -0.1 & 1.00 \\
\hline 0.21 & 18. & 0.203 & 21883. & 3141. & 293.7 & -0.1 & 1.00 \\
\hline 0.21 & 18. & 0.203 & 21928. & 3211. & 293.7 & -0.1 & 1.00 \\
\hline 0.21 & 18. & 0.203 & 21971. & 3273. & 293.7 & 0.0 & 1.00 \\
\hline 0.21 & 18. & 0.203 & 22017. & 3183. & 293.7 & 0.0 & 1.00 \\
\hline 0.21 & 18. & 0.203 & 22063. & 3229. & 293.6 & 0.0 & 1.00 \\
\hline 0.21 & 18. & 0.203 & 22112. & 3112. & 293.6 & 0.0 & 1.00 \\
\hline 0.21 & 18. & 0.203 & 22163. & 3241. & 293.6 & 0.1 & 1.00 \\
\hline 0.21 & 18. & 0.203 & 22211. & 3206. & 293.6 & 0.1 & 1.00 \\
\hline 0.21 & 18. & 0.203 & 22255 . & 3093. & 293.6 & 0.1 & 1.00 \\
\hline 0.21 & 18. & 0.203 & 22299. & 3228. & 293.5 & 0.1 & 1.00 \\
\hline 0.21 & 18. & 0.203 & 22343. & 3139. & 293.5 & 0.2 & 1.00 \\
\hline 0.21 & 18. & 0.203 & 22385. & 3133. & 293.5 & 0.1 & 1.00 \\
\hline 0.15 & 13. & 0.220 & 26466. & 3174. & 293.9 & -0.2 & 1.00 \\
\hline 0.15 & 13. & 0.220 & 26517. & 3158. & 293.9 & -0.2 & 1.00 \\
\hline 0.15 & 13. & 0.220 & 26566. & 3138. & 293.8 & -0.1 & 1.00 \\
\hline 0.15 & 13. & 0.220 & 26615. & 3256. & 293.8 & -0.1 & 1.00 \\
\hline 0.15 & 13. & 0.220 & 26661. & 3280. & 293.8 & $\begin{array}{l}-0.1 \\
\end{array}$ & 1.00 \\
\hline 0.15 & 13. & 0.220 & 26702 . & 3254. & 293.8 & -0.1 & 1.00 \\
\hline 0.15 & 13. & 0.220 & 26748. & 3134. & 293.7 & -0.1 & 1.00 \\
\hline 0.15 & 13. & 0.220 & 26794. & 3140. & 293.7 & -0.1 & 1.00 \\
\hline 0.15 & 13. & 0.220 & 26841. & 3131. & 293.7 & 0.0 & 1.00 \\
\hline 0.15 & 13. & 0.220 & 26887. & 3145. & 293.7 & 0.0 & 1.00 \\
\hline 0.15 & 13. & 0.220 & 26934. & 3197. & 293.6 & 0.0 & 1.00 \\
\hline 0.15 & 13. & 0.220 & 26978. & 3179. & 293.6 & 0.0 & 1.00 \\
\hline 0.15 & 13. & 0.220 & 27023. & 3118. & 293.6 & 0.0 & 1.00 \\
\hline 1.79 & 152. & 0.283 & 349347. & 3146. & 293.9 & -0.3 & 1.00 \\
\hline 1.79 & 152. & 0.283 & 349389. & 3169. & 293.9 & -0.3 & 1.00 \\
\hline 1.79 & 152. & 0.283 & 349434. & 3162. & 294.0 & -0.3 & 1.00 \\
\hline 1.79 & 152. & 0.283 & 349478. & 3341. & 294.0 & -0.3 & 1.00 \\
\hline 1.79 & 152. & 0.283 & 349522. & 3292. & 294.0 & $\begin{array}{l}-0.3 \\
\end{array}$ & 1.00 \\
\hline 1.79 & 152. & 0.283 & 349569. & 3260. & 294.0 & -0.3 & 1.00 \\
\hline 1.79 & 152. & 0.283 & 349620. & 3236. & 294.0 & $\begin{array}{l}-0.3 \\
\end{array}$ & 1.00 \\
\hline 1.79 & 152. & 0.283 & 349662. & 3318. & 294.0 & $\begin{array}{l}-0.3 \\
\end{array}$ & 1.00 \\
\hline 1.79 & 152. & 0.283 & 349706. & 3285. & 294.0 & -0.3 & 1.00 \\
\hline 1.79 & 152. & 0.283 & 349750. & 3293. & 294.0 & $\begin{array}{l}-0.3 \\
\end{array}$ & 1.00 \\
\hline 1.79 & 152. & 0.283 & 349792. & 3310. & 294.0 & -0.3 & 1.00 \\
\hline 1.79 & 152. & 0.283 & 349836. & 3336. & 294.0 & $\begin{array}{c}-0.3 \\
\end{array}$ & 1.00 \\
\hline
\end{tabular}


Table H.2.4 Diesel contamination on oxidized copper surface for $\operatorname{Re}=4600$ and $x_{\mathrm{b}}=0.2 \%$ (file:trv45con1.tb2)

\begin{tabular}{|c|c|c|c|c|c|c|c|}
\hline $\begin{array}{c}l_{\mathrm{e}} \\
(\mu \mathrm{m})\end{array}$ & $\begin{array}{c}\Gamma \\
\left(\mathrm{kg} / \mathrm{m}^{2}\right) \\
\mathrm{X} 10^{5}\end{array}$ & $\begin{array}{c}x_{\mathrm{b}} \\
(\%)\end{array}$ & $\begin{array}{l}\text { Exposure } \\
\text { Time (s) }\end{array}$ & $\mathrm{Re}$ & $\begin{array}{l}{\overline{T_{\mathrm{T}}}} \\
(\mathrm{K})\end{array}$ & $\begin{array}{c}T_{\mathrm{b}}-\bar{T}_{\mathrm{T}_{\mathrm{T}}} \\
(\mathrm{K})\end{array}$ & $\frac{F_{T_{\mathrm{b}}}}{F_{T_{\mathrm{T}}}}$ \\
\hline 7.96 & 676. & 0.171 & 236041. & 4684. & 293.4 & 0.3 & 1.00 \\
\hline 7.96 & 676. & 0.171 & 236081. & 4736. & 293.4 & 0.3 & 1.00 \\
\hline 7.96 & 676. & 0.171 & 236122. & 4503. & 293.4 & 0.3 & 1.00 \\
\hline 7.96 & 676. & 0.171 & 236166 . & 4684. & 293.4 & 0.3 & 1.00 \\
\hline 7.96 & 676. & 0.171 & 236207. & 4651. & 293.4 & 0.3 & 1.00 \\
\hline 7.96 & 676. & 0.171 & 236249 . & 4601. & 293.4 & 0.3 & 1.00 \\
\hline 7.96 & 676. & 0.171 & 236293. & 4636. & 293.4 & 0.3 & 1.00 \\
\hline 7.96 & 676. & 0.171 & 236338. & 4459. & 293.4 & 0.2 & 1.00 \\
\hline 7.96 & 676. & 0.171 & 236382. & 4654. & 293.4 & 0.2 & 1.00 \\
\hline 7.96 & 676. & 0.171 & 236423. & 4524. & 293.4 & 0.2 & 1.00 \\
\hline 7.96 & 676. & 0.171 & 236464. & 4572. & 293.4 & 0.2 & 1.00 \\
\hline 7.96 & 676. & 0.171 & 236505. & 4480 . & 293.4 & 0.2 & 1.00 \\
\hline 7.96 & 676. & 0.171 & 236554. & 4624. & 293.4 & 0.2 & 1.00 \\
\hline 7.89 & 670. & 0.170 & 236711. & 4568. & 293.5 & 0.1 & 1.00 \\
\hline 7.89 & 670. & 0.170 & 236754. & 4634. & 293.5 & 0.1 & 1.00 \\
\hline 7.89 & 670. & 0.170 & 236797. & 4571. & 293.6 & 0.1 & 1.00 \\
\hline 7.89 & 670. & 0.170 & 236838. & 4573. & 293.6 & 0.1 & 1.00 \\
\hline 7.89 & 670. & 0.170 & 236883. & 4593. & 293.6 & 0.0 & 1.00 \\
\hline 7.89 & 670. & 0.170 & 236931. & 4514. & 293.6 & 0.0 & 1.00 \\
\hline 7.89 & 670. & 0.170 & 236982. & 4500. & 293.6 & 0.0 & 1.00 \\
\hline 7.89 & 670 . & 0.170 & 237026 . & 4440. & 293.7 & 0.0 & 1.00 \\
\hline 7.89 & 670. & 0.170 & 237070 . & 4736. & 293.7 & -0.1 & 1.00 \\
\hline 7.89 & 670. & 0.170 & 237111. & 4477. & 293.7 & -0.1 & 1.00 \\
\hline 7.89 & 670. & 0.170 & 237152. & 4465. & 293.7 & -0.1 & 1.00 \\
\hline 7.89 & 670. & 0.170 & 237198. & 4628. & 293.8 & -0.1 & 1.00 \\
\hline 7.89 & 670. & 0.170 & 237241. & 4800 . & 293.8 & -0.1 & 1.00 \\
\hline 7.83 & 665. & 0.155 & 240174. & 4594. & 293.4 & 0.2 & 1.00 \\
\hline 7.83 & 665. & 0.155 & 240224. & 4495. & 293.4 & 0.2 & 1.00 \\
\hline 7.83 & 665. & 0.155 & 240267. & 4491. & 293.4 & 0.2 & 1.00 \\
\hline 7.83 & 665. & 0.155 & 240313. & 4523. & 293.4 & 0.3 & 1.00 \\
\hline 7.83 & 665. & 0.155 & 240355. & 4505. & 293.4 & 0.3 & 1.00 \\
\hline 7.83 & 665. & 0.155 & 240396. & 4491. & 293.4 & 0.3 & 1.00 \\
\hline 7.83 & 665. & 0.155 & 240460 . & 4650. & 293.4 & 0.3 & 1.00 \\
\hline 7.83 & 665. & 0.155 & 240502 . & 4652. & 293.4 & 0.3 & 1.00 \\
\hline 7.83 & 665. & 0.155 & 240546. & 4504. & 293.4 & 0.3 & 1.00 \\
\hline 7.83 & 665. & 0.155 & 240591. & 4633. & 293.4 & 0.3 & 1.00 \\
\hline 7.83 & 665. & 0.155 & 240635 . & 4460. & 293.4 & 0.3 & 1.00 \\
\hline 7.83 & 665. & 0.155 & 240679. & 4587. & 293.4 & 0.3 & 1.00 \\
\hline 7.83 & 665. & 0.155 & 240723. & 4429. & 293.4 & 0.3 & 1.00 \\
\hline 7.79 & 661. & 0.150 & 243116. & 4657. & 294.0 & -0.4 & 1.00 \\
\hline 7.79 & 661. & 0.150 & 243163. & 4791. & 294.0 & -0.4 & 1.00 \\
\hline 7.79 & 661. & 0.150 & 243213. & 4704. & 294.0 & -0.4 & 1.00 \\
\hline 7.79 & 661. & 0.150 & 243257. & 4619. & 294.0 & -0.4 & 1.00 \\
\hline 7.79 & 661. & 0.150 & 243301. & 4444. & 294.0 & -0.3 & 1.00 \\
\hline 7.79 & 661. & 0.150 & 243345 . & 4649. & 294.0 & -0.3 & 1.00 \\
\hline 7.79 & 661. & 0.150 & 243390. & 4456. & 294.0 & -0.3 & 1.00 \\
\hline 7.79 & 661. & 0.150 & 243433. & 4780. & 293.9 & -0.3 & 1.00 \\
\hline 7.79 & 661. & 0.150 & 243474. & 4711. & 293.9 & -0.3 & 1.00 \\
\hline 7.79 & 661. & 0.150 & 243515. & 4693. & 293.9 & -0.3 & 1.00 \\
\hline 7.79 & 661. & 0.150 & 243556. & 4624. & 293.9 & -0.3 & 1.00 \\
\hline 7.79 & 661. & 0.150 & 243596. & 4527. & 293.9 & -0.3 & 1.00 \\
\hline 7.79 & 661. & 0.150 & 243639. & 4654. & 293.9 & -0.2 & 1.00 \\
\hline 8.05 & 684. & 0.121 & 243799. & 4667. & 293.8 & -0.2 & 1.00 \\
\hline 8.05 & 684. & 0.121 & 243844. & 4615. & 293.8 & -0.1 & 1.00 \\
\hline 8.05 & 684. & 0.121 & 243886. & 4662. & 293.8 & -0.1 & 1.00 \\
\hline 8.05 & 684. & 0.121 & 243929. & 4563. & 293.8 & -0.1 & 1.00 \\
\hline 8.05 & 684. & 0.121 & 243972. & 4658. & 293.7 & -0.1 & 1.00 \\
\hline 8.05 & 684. & 0.121 & 244014. & 4724. & 293.7 & -0.1 & 1.00 \\
\hline 8.05 & 684. & 0.121 & 244056 . & 4671. & 293.7 & -0.1 & 1.00 \\
\hline 8.05 & 684. & 0.121 & 244095. & 4445. & 293.7 & 0.0 & 1.00 \\
\hline 8.05 & 684. & 0.121 & 244138. & 4583. & 293.6 & 0.0 & 1.00 \\
\hline 8.05 & 684. & 0.121 & 244181. & 4441. & 293.6 & 0.0 & 1.00 \\
\hline 8.05 & 684. & 0.121 & 244226 . & 4547. & 293.6 & 0.0 & 1.00 \\
\hline 8.05 & 684. & 0.121 & 244268. & 4625. & 293.6 & 0.1 & 1.00 \\
\hline 8.05 & 684. & 0.121 & 244315. & 4496. & 293.6 & 0.1 & 1.00 \\
\hline 7.85 & 667. & 0.152 & 244469. & 4473. & 293.5 & 0.1 & 1.00 \\
\hline 7.85 & 667. & 0.152 & 244510. & 4548. & 293.5 & 0.2 & 1.00 \\
\hline 7.85 & 667. & 0.152 & 244553. & 4544. & 293.5 & 0.2 & 1.00 \\
\hline 7.85 & 667. & 0.152 & 244598. & 4624. & 293.4 & 0.2 & 1.00 \\
\hline 7.85 & 667. & 0.152 & 244644. & 4403. & 293.4 & 0.2 & 1.00 \\
\hline 7.85 & 667. & 0.152 & 244685 . & 4671. & 293.4 & 0.2 & 1.00 \\
\hline 7.85 & 667. & 0.152 & 244744 . & 4570. & 293.4 & 0.2 & 1.00 \\
\hline 7.85 & 667. & 0.152 & 244791. & 4636. & 293.4 & 0.2 & 1.00 \\
\hline 7.85 & 667. & 0.152 & 244838. & 4601. & 293.4 & 0.3 & 1.00 \\
\hline 7.85 & 667. & 0.152 & 244885 . & 4617. & 293.4 & 0.3 & 1.00 \\
\hline
\end{tabular}

\begin{tabular}{|c|c|c|c|c|c|c|c|}
\hline 7.85 & 667. & 0.152 & 244930. & 4490. & 293.4 & 0.3 & 1.00 \\
\hline 7.85 & 667. & 0.152 & 244975. & 4649. & 293.4 & 0.3 & 1.00 \\
\hline 7.85 & 667. & 0.152 & 245019. & 4668. & 293.4 & 0.3 & 1.00 \\
\hline 7.84 & 666. & 0.118 & 248265. & 4789. & 293.8 & -0.2 & 1.00 \\
\hline 7.84 & 666. & 0.118 & 248305. & 4685. & 293.8 & -0.1 & 1.00 \\
\hline 7.84 & 666. & 0.118 & 248349. & 4648. & 293.8 & -0.1 & 1.00 \\
\hline 7.84 & 666. & 0.118 & 248392. & 4632. & 293.8 & -0.1 & 1.00 \\
\hline 7.84 & 666. & 0.118 & 248436 . & 4626. & 293.7 & -0.1 & 1.00 \\
\hline 7.84 & 666. & 0.118 & 248482. & 4743. & 293.7 & -0.1 & 1.00 \\
\hline 7.84 & 666. & 0.118 & 248524. & 4707. & 293.7 & -0.1 & 1.00 \\
\hline 7.84 & 666. & 0.118 & 248566. & 4555. & 293.7 & 0.0 & 1.00 \\
\hline 7.84 & 666. & 0.118 & 248610. & 4669. & 293.7 & 0.0 & 1.00 \\
\hline 7.84 & 666. & 0.118 & 248650. & 4632. & 293.6 & 0.0 & 1.00 \\
\hline 7.84 & 666. & 0.118 & 248697. & 4697. & 293.6 & 0.0 & 1.00 \\
\hline 7.84 & 666. & 0.118 & 248739. & 4680 . & 293.6 & 0.0 & 1.00 \\
\hline 7.84 & 666. & 0.118 & 248783. & 4545. & 293.6 & 0.1 & 1.00 \\
\hline 7.50 & 637. & 0.125 & 255063. & 4646. & 293.8 & -0.1 & 1.00 \\
\hline 7.50 & 637. & 0.125 & 255106. & 4458. & 293.8 & -0.1 & 1.00 \\
\hline 7.50 & 637. & 0.125 & 255151. & 4635. & 293.8 & -0.2 & 1.00 \\
\hline 7.50 & 637. & 0.125 & 255199. & 4707. & 293.9 & -0.2 & 1.00 \\
\hline 7.50 & 637. & 0.125 & 255243. & 4743. & 293.9 & -0.2 & 1.00 \\
\hline 7.50 & 637. & 0.125 & 255284. & 4676. & 293.9 & -0.3 & 1.00 \\
\hline 7.50 & 637. & 0.125 & 255327. & 4598. & 293.9 & -0.3 & 1.00 \\
\hline 7.50 & 637. & 0.125 & 255369. & 4698. & 293.9 & -0.3 & 1.00 \\
\hline 7.50 & 637. & 0.125 & 255413. & 4719. & 294.0 & -0.3 & 1.00 \\
\hline 7.50 & 637. & 0.125 & 255457. & 4705 . & 294.0 & $\begin{array}{l}-0.3 \\
\end{array}$ & 1.00 \\
\hline 7.50 & 637. & 0.125 & 255502. & 4687. & 294.0 & -0.4 & 1.00 \\
\hline 7.50 & 637. & 0.125 & 255544. & 4607. & 294.0 & -0.4 & 1.00 \\
\hline 7.50 & 637. & 0.125 & 255586. & 4640. & 294.0 & -0.4 & 1.00 \\
\hline 7.40 & 628. & 0.151 & 261040. & 4483. & 294.1 & -0.4 & 1.00 \\
\hline 7.40 & 628. & 0.151 & 261080. & 4743. & 294.1 & -0.4 & 1.00 \\
\hline 7.40 & 628. & 0.151 & 261120. & 4496. & 294.0 & -0.4 & 1.00 \\
\hline 7.40 & 628. & 0.151 & 261162. & 4740. & 294.0 & -0.4 & 1.00 \\
\hline 7.40 & 628. & 0.151 & 261204. & 4705 . & 294.0 & -0.4 & 1.00 \\
\hline 7.40 & 628. & 0.151 & 261245. & 4462. & 294.0 & -0.4 & 1.00 \\
\hline 7.40 & 628. & 0.151 & 261287. & 4618. & 294.0 & $\begin{array}{l}-0.3 \\
\end{array}$ & 1.00 \\
\hline 7.40 & 628. & 0.151 & 261331. & 4584. & 294.0 & -0.3 & 1.00 \\
\hline 7.40 & 628. & 0.151 & 261373. & 4666. & 294.0 & -0.3 & 1.00 \\
\hline 7.40 & 628. & 0.151 & 261412. & 4766. & 293.9 & $\begin{array}{l}-0.3 \\
\end{array}$ & 1.00 \\
\hline 7.40 & 628. & 0.151 & 261453. & 4712. & 293.9 & -0.3 & 1.00 \\
\hline 7.40 & 628. & 0.151 & 261494. & 4694. & 293.9 & -0.3 & 1.00 \\
\hline 7.40 & 628. & 0.151 & 261535. & 4544. & 293.9 & -0.3 & 1.00 \\
\hline 7.51 & 637. & 0.152 & 261665 . & 4638. & 293.9 & -0.2 & 1.00 \\
\hline 7.51 & 637. & 0.152 & 261705 . & 4572. & 293.9 & -0.2 & 1.00 \\
\hline 7.51 & 637. & 0.152 & 261748. & 4704. & 293.9 & -0.2 & 1.00 \\
\hline 7.51 & 637. & 0.152 & 261789. & 4504. & 293.8 & -0.2 & 1.00 \\
\hline 7.51 & 637. & 0.152 & 261828 . & 4633. & 293.8 & -0.2 & 1.00 \\
\hline 7.51 & 637. & 0.152 & 261871. & 4632. & 293.8 & -0.1 & 1.00 \\
\hline 7.51 & 637. & 0.152 & 261914. & 4498. & 293.8 & -0.1 & 1.00 \\
\hline 7.51 & 637. & 0.152 & 261955. & 4727. & 293.8 & -0.1 & 1.00 \\
\hline 7.51 & 637. & 0.152 & 262001. & 4404. & 293.7 & -0.1 & 1.00 \\
\hline 7.51 & 637. & 0.152 & 262045. & 4557. & 293.7 & -0.1 & 1.00 \\
\hline 7.51 & 637. & 0.152 & 262087. & 4414. & 293.7 & -0.1 & 1.00 \\
\hline 7.51 & 637. & 0.152 & 262129. & 4460. & 293.7 & 0.0 & 1.00 \\
\hline 7.51 & 637. & 0.152 & 262173. & 4487. & 293.6 & 0.0 & 1.00 \\
\hline 7.34 & 623. & 0.110 & 323906 . & 4531. & 294.1 & -0.4 & 1.00 \\
\hline 7.34 & 623. & 0.110 & 323949. & 4467. & 294.0 & -0.4 & 1.00 \\
\hline 7.34 & 623. & 0.110 & 323995. & 4674. & 294.0 & -0.4 & 1.00 \\
\hline 7.34 & 623. & 0.110 & 324038. & 4707. & 294.0 & -0.4 & 1.00 \\
\hline 7.34 & 623. & 0.110 & 324081. & 4623. & 294.0 & -0.4 & 1.00 \\
\hline 7.34 & 623. & 0.110 & 324121. & 4479. & 294.0 & -0.4 & 1.00 \\
\hline 7.34 & 623. & 0.110 & 324163. & 4687. & 294.0 & -0.4 & 1.00 \\
\hline 7.34 & 623. & 0.110 & 324202. & 4508. & 294.0 & -0.3 & 1.00 \\
\hline 7.34 & 623. & 0.110 & 324244. & 4476. & 294.0 & -0.3 & 1.00 \\
\hline 7.34 & 623. & 0.110 & 324285 . & 4489. & 293.9 & -0.3 & 1.00 \\
\hline 7.34 & 623. & 0.110 & 324327. & 4681. & 293.9 & $\begin{array}{l}-0.3 \\
\end{array}$ & 1.00 \\
\hline 7.34 & 623. & 0.110 & 324368. & 4411. & 293.9 & -0.3 & 1.00 \\
\hline 7.34 & 623. & 0.110 & 324412. & 4578. & 293.9 & -0.3 & 1.00 \\
\hline 7.08 & 601. & 0.150 & 327196. & 4693. & 293.9 & $\begin{array}{l}-0.3 \\
\end{array}$ & 1.00 \\
\hline 7.08 & 601. & 0.150 & 327249 . & 4612. & 293.9 & -0.3 & 1.00 \\
\hline 7.08 & 601. & 0.150 & 327299. & 4731. & 294.0 & -0.3 & 1.00 \\
\hline 7.08 & 601. & 0.150 & 327353. & 4444. & 294.0 & $\begin{array}{l}-0.3 \\
\end{array}$ & 1.00 \\
\hline 7.08 & 601. & 0.150 & 327404 . & 4652. & 294.0 & -0.4 & 1.00 \\
\hline 7.08 & 601. & 0.150 & 327448. & 4724. & 294.0 & -0.4 & 1.00 \\
\hline 7.08 & 601. & 0.150 & 327492. & 4724. & 294.0 & -0.4 & 1.00 \\
\hline 7.08 & 601. & 0.150 & 327535. & 4673. & 294.0 & -0.4 & 1.00 \\
\hline 7.08 & 601. & 0.150 & 327577. & 4437. & 294.1 & -0.4 & 1.00 \\
\hline 7.08 & 601. & 0.150 & 327620 . & 4529. & 294.1 & -0.4 & 1.00 \\
\hline
\end{tabular}


Table H.2.5 Diesel contamination on oxidized copper surface for $\operatorname{Re}=7000$ and $x_{\mathrm{b}}=0.2 \%$ (file:trv6con1.tb2)

\begin{tabular}{|c|c|c|c|c|c|c|c|}
\hline $\begin{array}{c}l_{\mathrm{e}} \\
(\mu \mathrm{m})\end{array}$ & $\begin{array}{c}\Gamma \\
\left(\mathrm{kg} / \mathrm{m}^{2}\right) \\
\mathrm{X} 10^{5}\end{array}$ & $\begin{array}{l}x_{\mathrm{b}} \\
(\%) \\
\end{array}$ & $\begin{array}{c}\text { Exposure } \\
\text { Time (s) }\end{array}$ & $\operatorname{Re}$ & $\begin{array}{l}\bar{T}_{\mathrm{T}_{\mathrm{T}}} \\
(\mathrm{K})\end{array}$ & $\begin{array}{c}T_{\mathrm{b}}-\bar{T}_{\mathrm{T}_{\mathrm{T}}} \\
(\mathrm{K})\end{array}$ & $\frac{F_{T_{b}}}{F_{T_{\mathrm{T}}}}$ \\
\hline 0.08 & 7. & 0.207 & 4639. & 7434. & 297.5 & $\begin{array}{l}-3.8 \\
\end{array}$ & 0.99 \\
\hline 0.08 & 7. & 0.207 & 4684. & 7364 . & 297.5 & $\begin{array}{l}-3.8 \\
\end{array}$ & 0.99 \\
\hline 0.08 & 7. & 0.207 & 4728. & 7259 . & 297.5 & -3.9 & 0.99 \\
\hline 0.08 & 7. & 0.207 & 4771. & 7155. & 297.6 & -3.9 & 0.99 \\
\hline 0.08 & 7. & 0.207 & 4813. & 7463. & 297.6 & -4.0 & 0.99 \\
\hline 0.08 & 7. & 0.207 & 4857. & 7199. & 297.6 & -4.0 & 0.99 \\
\hline 0.08 & 7. & 0.207 & 4900. & 7548. & 297.7 & -4.0 & 0.99 \\
\hline 0.08 & 7. & 0.207 & 4943. & 7284. & 297.7 & -4.0 & 0.99 \\
\hline 0.08 & 7. & 0.207 & 4987. & 7368. & 297.7 & -4.1 & 0.99 \\
\hline 0.08 & 7. & 0.207 & 5029. & 7447. & 297.8 & $\begin{array}{l}-4.1 \\
\end{array}$ & 0.99 \\
\hline 0.08 & 7. & 0.207 & 5075. & 7297. & 297.8 & -4.1 & 0.99 \\
\hline 0.08 & 7. & 0.207 & 5122. & 7116. & 297.8 & -4.2 & 0.99 \\
\hline 0.08 & 7. & 0.207 & 5163. & 7618. & 297.8 & -4.2 & 0.99 \\
\hline 0.27 & 23. & 0.177 & 5325. & 7362. & 297.9 & -4.2 & 0.99 \\
\hline 0.27 & 23. & 0.177 & 5366. & 7248. & 297.9 & -4.3 & 0.99 \\
\hline 0.27 & 23. & 0.177 & 5412. & 7248. & 297.9 & -4.3 & 0.99 \\
\hline 0.27 & 23. & 0.177 & 5459. & 7285. & 297.9 & -4.3 & 0.99 \\
\hline 0.27 & 23. & 0.177 & 5511. & 7365. & 298.0 & -4.3 & 0.99 \\
\hline 0.27 & 23. & 0.177 & 5554. & 7522 . & 298.0 & -4.3 & 0.99 \\
\hline 0.27 & 23. & 0.177 & 5600. & 7564. & 298.0 & -4.3 & 0.99 \\
\hline 0.27 & 23. & 0.177 & 5642. & 7486. & 298.0 & -4.3 & 0.99 \\
\hline 0.27 & 23. & 0.177 & 5685. & 7219. & 298.0 & -4.3 & 0.99 \\
\hline 0.27 & 23. & 0.177 & 5726. & 7566. & 298.0 & -4.3 & 0.99 \\
\hline 0.27 & 23. & 0.177 & 5771. & 7526 . & 298.0 & $\begin{array}{l}-4.3 \\
\end{array}$ & 0.99 \\
\hline 0.27 & 23. & 0.177 & 5818. & 7567. & 298.0 & $\begin{array}{l}-4.3 \\
\end{array}$ & 0.99 \\
\hline 0.27 & 23. & 0.177 & 5865. & 7331. & 298.0 & $\begin{array}{l}-4.3 \\
\end{array}$ & 0.99 \\
\hline 0.56 & 48. & 0.180 & 7893. & 7458. & 297.4 & -3.7 & 0.99 \\
\hline 0.56 & 48. & 0.180 & 7942. & 7661. & 297.3 & -3.6 & 0.99 \\
\hline 0.56 & 48. & 0.180 & 7988. & 7298. & 297.3 & -3.6 & 0.99 \\
\hline 0.56 & 48. & 0.180 & 8031. & 7489. & 297.3 & $\begin{array}{l}-3.6 \\
\end{array}$ & 0.99 \\
\hline 0.56 & 48. & 0.180 & 8072. & 7564. & 297.3 & -3.6 & 0.99 \\
\hline 0.56 & 48. & 0.180 & 8115. & 7101. & 297.2 & -3.6 & 0.99 \\
\hline 0.56 & 48. & 0.180 & 8159. & 7359. & 297.2 & -3.5 & 0.99 \\
\hline 0.56 & 48. & 0.180 & 8202. & 7166. & 297.2 & -3.5 & 0.99 \\
\hline 0.56 & 48. & 0.180 & 8245. & 7311. & 297.2 & -3.5 & 0.99 \\
\hline 0.56 & 48. & 0.180 & 8296. & 7469. & 297.1 & -3.5 & 0.99 \\
\hline 0.56 & 48. & 0.180 & 8341. & 7309. & 297.1 & -3.5 & 0.99 \\
\hline 0.56 & 48. & 0.180 & 8392. & 7264. & 297.1 & -3.4 & 0.99 \\
\hline 0.56 & 48. & 0.180 & 8449. & 7455 . & 297.1 & -3.4 & 0.99 \\
\hline 0.86 & 73. & 0.209 & 11766. & 7187. & 295.4 & $\begin{array}{l}-1.8 \\
\end{array}$ & 1.00 \\
\hline 0.86 & 73. & 0.209 & 11806. & 7144. & 295.4 & -1.7 & 1.00 \\
\hline 0.86 & 73. & 0.209 & 11848. & 7255 . & 295.4 & -1.7 & 1.00 \\
\hline 0.86 & 73. & 0.209 & 11894. & 7252. & 295.4 & -1.7 & 1.00 \\
\hline 0.86 & 73. & 0.209 & 11937. & 6842. & 295.3 & -1.7 & 1.00 \\
\hline 0.86 & 73. & 0.209 & 11984. & 7015. & 295.3 & -1.6 & 1.00 \\
\hline 0.86 & 73. & 0.209 & 12032. & 6976. & 295.3 & -1.6 & 1.00 \\
\hline 0.86 & 73. & 0.209 & 12082. & 7198. & 295.3 & -1.6 & 1.00 \\
\hline 0.86 & 73. & 0.209 & 12124. & 7238. & 295.3 & $\begin{array}{l}-1.6 \\
\end{array}$ & 1.00 \\
\hline 0.86 & 73. & 0.209 & 12168. & 7153. & 295.2 & -1.6 & 1.00 \\
\hline 0.86 & 73. & 0.209 & 12208. & 6891. & 295.2 & -1.6 & 1.00 \\
\hline 0.86 & 73. & 0.209 & 12252. & 7033. & 295.2 & -1.5 & 1.00 \\
\hline 0.86 & 73. & 0.209 & 12298. & 7030 . & 295.2 & -1.5 & 1.00 \\
\hline 1.08 & 92. & 0.203 & 12461. & 6981. & 295.1 & -1.4 & 1.00 \\
\hline 1.08 & 92. & 0.203 & 12506. & 6870. & 295.1 & -1.4 & 1.00 \\
\hline 1.08 & 92. & 0.203 & 12550. & 7163. & 295.1 & $\begin{array}{l}-1.4 \\
\end{array}$ & 1.00 \\
\hline 1.08 & 92. & 0.203 & 12595. & 6797. & 295.0 & -1.4 & 1.00 \\
\hline 1.08 & 92. & 0.203 & 12637. & 6971. & 295.0 & -1.4 & 1.00 \\
\hline 1.08 & 92. & 0.203 & 12686. & 6654. & 295.0 & $\begin{array}{l}-1.3 \\
\end{array}$ & 1.00 \\
\hline 1.08 & 92. & 0.203 & 12730. & 6928. & 295.0 & $\begin{array}{l}-1.3 \\
\end{array}$ & 1.00 \\
\hline 1.08 & 92. & 0.203 & 12773. & 6852. & 294.9 & $\begin{array}{l}-1.3 \\
\end{array}$ & 1.00 \\
\hline 1.08 & 92. & 0.203 & 12819. & 6883. & 294.9 & $\begin{array}{l}-1.3 \\
\end{array}$ & 1.00 \\
\hline 1.08 & 92. & 0.203 & 12861. & 6989. & 294.9 & -1.2 & 1.00 \\
\hline 1.08 & 92. & 0.203 & 12903. & 6809. & 294.9 & -1.2 & 1.00 \\
\hline 1.08 & 92. & 0.203 & 12952. & 6945. & 294.9 & $\begin{array}{l}-1.2 \\
\end{array}$ & 1.00 \\
\hline 1.08 & 92. & 0.203 & 12994. & 6942. & 294.8 & -1.2 & 1.00 \\
\hline 1.14 & 96. & 0.208 & 13180. & 6967. & 294.8 & -1.1 & 1.00 \\
\hline 1.14 & 96. & 0.208 & 13223. & 7115. & 294.7 & -1.1 & 1.00 \\
\hline 1.14 & 96. & 0.208 & 13267. & 7189. & 294.7 & $\begin{array}{l}-1.1 \\
\end{array}$ & 1.00 \\
\hline 1.14 & 96. & 0.208 & 13309. & 6850. & 294.7 & -1.0 & 1.00 \\
\hline 1.14 & 96. & 0.208 & 13352. & 6744. & 294.7 & -1.0 & 1.00 \\
\hline 1.14 & 96. & 0.208 & 13394. & 7062. & 294.7 & -1.0 & 1.00 \\
\hline 1.14 & 96. & 0.208 & 13439. & 6987. & 294.6 & -1.0 & 1.00 \\
\hline 1.14 & 96. & 0.208 & 13482. & 6980. & 294.6 & -1.0 & 1.00 \\
\hline 1.14 & 96. & 0.208 & 13526. & 6872. & 294.6 & -0.9 & 1.00 \\
\hline 1.14 & 96. & 0.208 & 13569. & 7090 . & 294.6 & -0.9 & 1.00 \\
\hline
\end{tabular}

\begin{tabular}{|c|c|c|c|c|c|c|c|}
\hline 1.14 & 96. & 0.208 & 13615. & 7125 . & 294.6 & -0.9 & 1.00 \\
\hline 1.14 & 96. & 0.208 & 13668. & 6933. & 294.6 & -0.9 & 1.00 \\
\hline 1.14 & 96. & 0.208 & 13730 . & 7118. & 294.5 & -0.9 & 1.00 \\
\hline 1.92 & 163. & 0.204 & 17643. & 6787. & 293.9 & -0.2 & 1.00 \\
\hline 1.92 & 163. & 0.204 & 17687. & 7049 . & 293.9 & -0.2 & 1.00 \\
\hline 1.92 & 163. & 0.204 & 17730. & 7056. & 293.9 & -0.3 & 1.00 \\
\hline 1.92 & 163. & 0.204 & 17772. & 6981. & 293.9 & -0.3 & 1.00 \\
\hline 1.92 & 163. & 0.204 & 17813. & 6841. & 294.0 & -0.3 & 1.00 \\
\hline 1.92 & 163. & 0.204 & 17853. & 6917. & 294.0 & -0.3 & 1.00 \\
\hline 1.92 & 163. & 0.204 & 17900. & 6885. & 294.0 & -0.3 & 1.00 \\
\hline 1.92 & 163. & 0.204 & 17940. & 6676. & 294.0 & -0.4 & 1.00 \\
\hline 1.92 & 163. & 0.204 & 17982. & 7001. & 294.1 & -0.4 & 1.00 \\
\hline 1.92 & 163. & 0.204 & 18024. & 6967. & 294.1 & -0.4 & 1.00 \\
\hline 1.92 & 163. & 0.204 & 18067. & 6824. & 294.1 & -0.4 & 1.00 \\
\hline 1.92 & 163. & 0.204 & 18109. & 7048. & 294.1 & -0.4 & 1.00 \\
\hline 1.92 & 163. & 0.204 & 18154. & 7011. & 294.1 & -0.5 & 1.00 \\
\hline 4.07 & 346. & 0.070 & 22676. & 6728. & 293.6 & 0.1 & 1.00 \\
\hline 4.07 & 346. & 0.070 & 22720 . & 6628. & 293.6 & 0.0 & 1.00 \\
\hline 4.07 & 346. & 0.070 & 22763. & 6917. & 293.7 & 0.0 & 1.00 \\
\hline 4.07 & 346. & 0.070 & 22806 . & 6568. & 293.7 & 0.0 & 1.00 \\
\hline 4.07 & 346. & 0.070 & 22848 . & 6999. & 293.7 & -0.1 & 1.00 \\
\hline 4.07 & 346. & 0.070 & 22892. & 6929. & 293.7 & -0.1 & 1.00 \\
\hline 4.07 & 346. & 0.070 & 22939. & 6970. & 293.8 & -0.1 & 1.00 \\
\hline 4.07 & 346. & 0.070 & 22984. & 6903. & 293.8 & -0.1 & 1.00 \\
\hline 4.07 & 346. & 0.070 & 23032. & 6944. & 293.8 & -0.2 & 1.00 \\
\hline 4.07 & 346. & 0.070 & 23076 . & 7023. & 293.9 & -0.2 & 1.00 \\
\hline 4.07 & 346. & 0.070 & 23118. & 6600. & 293.9 & -0.2 & 1.00 \\
\hline 4.07 & 346. & 0.070 & 23160 . & 6916. & 293.9 & -0.3 & 1.00 \\
\hline 4.07 & 346. & 0.070 & 23203. & 6923. & 293.9 & -0.3 & 1.00 \\
\hline 2.63 & 224. & 0.111 & 26115. & 7046. & 293.8 & -0.1 & 1.00 \\
\hline 2.63 & 224. & 0.111 & 26160 . & 6857. & 293.8 & -0.1 & 1.00 \\
\hline 2.63 & 224. & 0.111 & 26208 . & 6818. & 293.8 & -0.1 & 1.00 \\
\hline 2.63 & 224. & 0.111 & 26261. & 6604. & 293.7 & -0.1 & 1.00 \\
\hline 2.63 & 224. & 0.111 & 26305. & 6706. & 293.7 & -0.1 & 1.00 \\
\hline 2.63 & 224. & 0.111 & 26354. & 6918. & 293.7 & 0.0 & 1.00 \\
\hline 2.63 & 224. & 0.111 & 26399. & 6843. & 293.7 & 0.0 & 1.00 \\
\hline 2.63 & 224. & 0.111 & 26442. & 6914. & 293.7 & 0.0 & 1.00 \\
\hline 2.63 & 224. & 0.111 & 26511. & 7020 . & 293.6 & 0.0 & 1.00 \\
\hline 2.63 & 224. & 0.111 & 26555. & 6986. & 293.6 & 0.0 & 1.00 \\
\hline 2.63 & 224. & 0.111 & 26600. & 6831. & 293.6 & 0.0 & 1.00 \\
\hline 2.63 & 224. & 0.111 & 26644. & 6939. & 293.6 & 0.1 & 1.00 \\
\hline 2.63 & 224. & 0.111 & 26688. & 7017. & 293.6 & 0.1 & 1.00 \\
\hline 1.41 & 119. & 0.220 & 26865. & 6740. & 293.6 & 0.1 & 1.00 \\
\hline 1.41 & 119. & 0.220 & 26911. & 6846. & 293.5 & 0.1 & 1.00 \\
\hline 1.41 & 119. & 0.220 & 26961. & 7030 . & 293.5 & 0.1 & 1.00 \\
\hline 1.41 & 119. & 0.220 & 27007. & 6911. & 293.5 & 0.2 & 1.00 \\
\hline 1.41 & 119. & 0.220 & 27054 . & 6951. & 293.5 & 0.2 & 1.00 \\
\hline 1.41 & 119. & 0.220 & 27097. & 6944. & 293.5 & 0.2 & 1.00 \\
\hline 1.41 & 119. & 0.220 & 27146. & 6766. & 293.5 & 0.2 & 1.00 \\
\hline 1.41 & 119. & 0.220 & 27192. & 6725 . & 293.5 & 0.2 & 1.00 \\
\hline 1.41 & 119. & 0.220 & 27237. & 7063. & 293.5 & 0.2 & 1.00 \\
\hline 1.41 & 119. & 0.220 & 27289. & 6623. & 293.5 & 0.2 & 1.00 \\
\hline 1.41 & 119. & 0.220 & 27333. & 6836. & 293.5 & 0.2 & 1.00 \\
\hline 1.41 & 119. & 0.220 & 27379. & 6691. & 293.5 & 0.2 & 1.00 \\
\hline 1.41 & 119. & 0.220 & 27435. & 6730. & 293.5 & 0.2 & 1.00 \\
\hline 0.94 & 79. & 0.216 & 88904. & 6650 . & 294.1 & -0.4 & 1.00 \\
\hline 0.94 & 79. & 0.216 & 88947. & 6578. & 294.1 & -0.4 & 1.00 \\
\hline 0.94 & 79. & 0.216 & 88989. & 6324. & 294.1 & -0.4 & 1.00 \\
\hline 0.94 & 79. & 0.216 & 89031. & 6677. & 294.1 & -0.4 & 1.00 \\
\hline 0.94 & 79. & 0.216 & 89073. & 6645. & 294.1 & -0.4 & 1.00 \\
\hline 0.94 & 79. & 0.216 & 89116. & 6481. & 294.0 & -0.4 & 1.00 \\
\hline 0.94 & 79. & 0.216 & 89164. & 6412. & 294.0 & -0.4 & 1.00 \\
\hline 0.94 & 79. & 0.216 & 89205. & 6605. & 294.0 & -0.4 & 1.00 \\
\hline 0.94 & 79. & 0.216 & 89246. & 6741. & 294.0 & -0.3 & 1.00 \\
\hline 0.94 & 79. & 0.216 & 89289. & 6473. & 294.0 & -0.4 & 1.00 \\
\hline 0.94 & 79. & 0.216 & 89333. & 6502. & 294.0 & -0.3 & 1.00 \\
\hline 0.94 & 79. & 0.216 & 89385. & 6536. & 294.0 & -0.3 & 1.00 \\
\hline 0.94 & 79. & 0.216 & 89435. & 6468. & 294.0 & -0.3 & 1.00 \\
\hline 1.07 & 91. & 0.220 & 93246. & 6237. & 294.1 & -0.4 & 1.00 \\
\hline 1.07 & 91. & 0.220 & 93287. & 6716. & 294.1 & -0.4 & 1.00 \\
\hline 1.07 & 91. & 0.220 & 93328. & 6648. & 294.1 & -0.4 & 1.00 \\
\hline 1.07 & 91. & 0.220 & 93369. & 6614. & 294.1 & -0.4 & 1.00 \\
\hline 1.07 & 91. & 0.220 & 93409. & 6614. & 294.1 & -0.4 & 1.00 \\
\hline 1.07 & 91. & 0.220 & 93456. & 6783. & 294.1 & -0.4 & 1.00 \\
\hline 1.07 & 91. & 0.220 & 93500. & 6680 . & 294.1 & -0.4 & 1.00 \\
\hline 1.07 & 91. & 0.220 & 93543. & 6416. & 294.1 & -0.4 & 1.00 \\
\hline 1.07 & 91. & 0.220 & 93588. & 6892. & 294.1 & -0.4 & 1.00 \\
\hline 1.07 & 91. & 0.220 & 93629. & 6477. & 294.0 & -0.4 & 1.00 \\
\hline
\end{tabular}


Table H.2.6 Tap water flushing after $\mathrm{Re}=4600$ contamination tests at $x_{\mathrm{b}}=0.2 \%$ (file:flsh45c1.tb2)

\begin{tabular}{|c|c|c|c|c|c|c|}
\hline $\begin{array}{c}\begin{array}{c}l_{\mathrm{e}} \\
(\mu \mathrm{m})\end{array} \\
\text { (a) }\end{array}$ & $\begin{array}{c}\Gamma \\
\left(\mathrm{kg} / \mathrm{m}^{2}\right) \\
\mathrm{X} 10^{5}\end{array}$ & $\begin{array}{l}x_{\mathrm{b}} \\
(\%)\end{array}$ & $\begin{array}{c}\text { Exposure } \\
\text { Time (s) }\end{array}$ & $\begin{array}{l}\overline{\bar{T}_{\mathrm{T}_{\mathrm{T}}}} \\
(\mathrm{K})\end{array}$ & $\begin{array}{c}T_{\mathrm{t}}-\bar{T}_{\mathrm{T}_{\mathrm{T}}} \\
(\mathrm{K})\end{array}$ & $\frac{F_{T_{T_{b}}}}{F_{T_{T_{\mathrm{T}}}}}$ \\
\hline 6.62 & 560. & 0.077 & 2413. & 300.1 & -6.5 & 0.99 \\
\hline 6.62 & 560. & 0.077 & 2467. & 299.9 & -6.4 & 0.99 \\
\hline 6.62 & 560. & 0.077 & 2522. & 299.9 & -6.3 & 0.99 \\
\hline 6.62 & 560. & 0.077 & 2574. & 299.9 & -6.3 & 0.99 \\
\hline 6.62 & 560. & 0.077 & 2634. & 299.8 & -6.3 & 0.99 \\
\hline 6.62 & 560. & 0.077 & 2686. & 299.8 & -6.2 & 0.99 \\
\hline 6.62 & 560. & 0.077 & 2744. & 299.7 & -6.2 & 0.99 \\
\hline 6.62 & 560. & 0.077 & 2796. & 299.7 & -6.1 & 0.99 \\
\hline 6.62 & 560. & 0.077 & 2849. & 299.6 & $\begin{array}{l}-6.0 \\
\end{array}$ & 0.99 \\
\hline 6.62 & 560. & 0.077 & 2898. & 299.5 & -5.9 & 0.99 \\
\hline 6.62 & 560. & 0.077 & 2953. & 299.4 & -5.8 & 0.99 \\
\hline 6.62 & 560. & 0.077 & 3000 . & 299.3 & -5.8 & 0.99 \\
\hline 6.02 & 509. & 0.037 & 3214. & 299.1 & -5.5 & 0.99 \\
\hline 6.02 & 509. & 0.037 & 3271. & 299.0 & -5.5 & 0.99 \\
\hline 6.02 & 509. & 0.037 & 3326. & 298.9 & -5.4 & 0.99 \\
\hline 6.02 & 509. & 0.037 & 3380. & 298.9 & $\begin{array}{l}-5.3 \\
\end{array}$ & 0.99 \\
\hline 6.02 & 509. & 0.037 & 3430. & 298.8 & -5.3 & 0.99 \\
\hline 6.02 & 509. & 0.037 & 3481. & 298.8 & -5.2 & 0.99 \\
\hline 6.02 & 509. & 0.037 & 3530. & 298.7 & -5.2 & 0.99 \\
\hline 6.02 & 509. & 0.037 & 3581. & 298.7 & -5.1 & 0.99 \\
\hline 6.02 & 509. & 0.037 & 3631. & 298.6 & -5.1 & 0.99 \\
\hline 6.02 & 509. & 0.037 & 3684. & 298.6 & -5.0 & 0.99 \\
\hline 4.89 & 416. & 0.040 & 25131. & 292.7 & 0.8 & 1.00 \\
\hline 4.89 & 416. & 0.040 & 25189. & 292.7 & 0.8 & 1.00 \\
\hline 4.89 & 416. & 0.040 & 25244. & 292.7 & 0.8 & 1.00 \\
\hline 4.89 & 416. & 0.040 & 25297. & 292.7 & 0.8 & 1.00 \\
\hline 4.89 & 416. & 0.040 & 25351. & 292.8 & 0.8 & 1.00 \\
\hline 4.89 & 416. & 0.040 & 25403. & 292.8 & 0.8 & 1.00 \\
\hline 4.89 & 416. & 0.040 & 25455. & 292.8 & 0.8 & 1.00 \\
\hline 4.89 & 416. & 0.040 & 25506. & 292.8 & 0.8 & 1.00 \\
\hline 4.89 & 416. & 0.040 & 25557. & 292.8 & 0.8 & 1.00 \\
\hline 4.88 & 415. & 0.047 & 25789. & 292.8 & 0.7 & 1.00 \\
\hline 4.88 & 415. & 0.047 & 25844. & 292.8 & 0.7 & 1.00 \\
\hline 4.88 & 415. & 0.047 & 25894. & 292.9 & 0.7 & 1.00 \\
\hline 4.88 & 415. & 0.047 & 25951. & 292.9 & 0.7 & 1.00 \\
\hline 4.88 & 415. & 0.047 & 26010. & 292.9 & 0.7 & 1.00 \\
\hline 4.88 & 415. & 0.047 & 26058. & 292.9 & 0.7 & 1.00 \\
\hline 4.88 & 415. & 0.047 & 26106. & 292.9 & 0.7 & 1.00 \\
\hline 3.29 & 280. & 0.009 & 83821. & 293.9 & $\begin{array}{l}-0.3 \\
\end{array}$ & 1.00 \\
\hline 3.29 & 280. & 0.009 & 84083. & 293.9 & -0.4 & 1.00 \\
\hline 3.29 & 280. & 0.009 & 84145. & 293.9 & -0.4 & 1.00 \\
\hline 3.29 & 280. & 0.009 & 84204. & 293.9 & -0.4 & 1.00 \\
\hline 3.29 & 280. & 0.009 & 84256. & 293.9 & -0.4 & 1.00 \\
\hline 3.29 & 280. & 0.009 & 84311. & 293.9 & -0.4 & 1.00 \\
\hline 3.29 & 280. & 0.009 & 84369. & 293.9 & -0.4 & 1.00 \\
\hline 3.29 & 280. & 0.009 & 84456. & 293.9 & -0.4 & 1.00 \\
\hline 3.29 & 280. & 0.009 & 84513. & 293.9 & -0.3 & 1.00 \\
\hline 3.29 & 280. & 0.009 & 84590. & 293.9 & -0.4 & 1.00 \\
\hline 3.29 & 280. & 0.009 & 84641. & 293.9 & -0.4 & 1.00 \\
\hline 3.29 & 280. & 0.009 & 84697. & 293.9 & -0.4 & 1.00 \\
\hline 3.29 & 280. & 0.009 & 84755. & 293.9 & -0.4 & 1.00 \\
\hline 3.27 & 278. & 0.022 & 85420. & 294.0 & -0.5 & 1.00 \\
\hline 3.27 & 278. & 0.022 & 85482. & 294.0 & -0.5 & 1.00 \\
\hline 3.27 & 278. & 0.022 & 85542. & 294.0 & -0.5 & 1.00 \\
\hline 3.27 & 278. & 0.022 & 85595. & 294.0 & -0.5 & 1.00 \\
\hline 3.27 & 278. & 0.022 & 85648. & 294.0 & -0.5 & 1.00 \\
\hline 3.27 & 278. & 0.022 & 85697. & 294.0 & -0.5 & 1.00 \\
\hline
\end{tabular}

\begin{tabular}{|c|c|c|c|c|c|c|}
\hline 3.27 & 278. & 0.022 & 85750. & 294.1 & -0.5 & 1.00 \\
\hline 3.27 & 278. & 0.022 & 85808. & 294.0 & -0.5 & 1.00 \\
\hline 3.27 & 278. & 0.022 & 85861. & 294.1 & -0.5 & 1.00 \\
\hline 3.27 & 278. & 0.022 & 85913. & 294.0 & -0.5 & 1.00 \\
\hline 3.27 & 278. & 0.022 & 85962. & 294.0 & -0.5 & 1.00 \\
\hline 3.27 & 278. & 0.022 & 86023. & 294.1 & -0.5 & 1.00 \\
\hline 3.27 & 278. & 0.022 & 86073. & 294.1 & -0.5 & 1.00 \\
\hline 2.46 & 209. & 0.012 & 111672. & 294.0 & -0.5 & 1.00 \\
\hline 2.46 & 209. & 0.012 & 111735. & 294.0 & -0.5 & 1.00 \\
\hline 2.46 & 209. & 0.012 & 111793. & 294.0 & -0.5 & 1.00 \\
\hline 2.46 & 209. & 0.012 & 111862. & 294.0 & -0.5 & 1.00 \\
\hline 2.46 & 209. & 0.012 & 111923. & 294.0 & -0.5 & 1.00 \\
\hline 2.46 & 209. & 0.012 & 111976. & 294.0 & -0.5 & 1.00 \\
\hline 2.46 & 209. & 0.012 & 112026. & 294.0 & -0.5 & 1.00 \\
\hline 2.46 & 209. & 0.012 & 112079. & 294.0 & -0.5 & 1.00 \\
\hline 2.46 & 209. & 0.012 & 112131. & 294.0 & -0.5 & 1.00 \\
\hline 2.46 & 209. & 0.012 & 112178. & 294.0 & -0.5 & 1.00 \\
\hline 2.46 & 209. & 0.012 & 112226. & 294.0 & -0.5 & 1.00 \\
\hline 2.46 & 209. & 0.012 & 112269. & 294.0 & -0.5 & 1.00 \\
\hline 2.46 & 209. & 0.012 & 112318. & 294.0 & -0.5 & 1.00 \\
\hline 2.50 & 212. & 0.020 & 112462. & 294.0 & -0.5 & 1.00 \\
\hline 2.50 & 212. & 0.020 & 112513. & 294.0 & -0.5 & 1.00 \\
\hline 2.50 & 212. & 0.020 & 112562. & 294.0 & -0.5 & 1.00 \\
\hline 2.50 & 212. & 0.020 & 112607. & 294.0 & -0.5 & 1.00 \\
\hline 2.50 & 212. & 0.020 & 112651. & 294.0 & -0.5 & 1.00 \\
\hline 1.94 & 165. & 0.014 & 169806. & 295.0 & -1.4 & 1.00 \\
\hline 1.94 & 165. & 0.014 & 169865. & 295.0 & -1.4 & 1.00 \\
\hline 1.94 & 165. & 0.014 & 169922. & 295.0 & -1.4 & 1.00 \\
\hline 1.94 & 165. & 0.014 & 169977. & 294.9 & -1.4 & 1.00 \\
\hline 1.94 & 165. & 0.014 & 170036. & 295.0 & -1.4 & 1.00 \\
\hline 1.94 & 165. & 0.014 & 170093. & 294.9 & -1.4 & 1.00 \\
\hline 1.94 & 165. & 0.014 & 170147. & 295.0 & -1.4 & 1.00 \\
\hline 1.94 & 165. & 0.014 & 170205. & 294.9 & -1.4 & 1.00 \\
\hline 1.94 & 165. & 0.014 & 170261. & 295.0 & -1.4 & 1.00 \\
\hline 1.94 & 165. & 0.014 & 170312. & 294.9 & -1.4 & 1.00 \\
\hline 1.94 & 165. & 0.014 & 170367. & 295.0 & -1.4 & 1.00 \\
\hline 1.94 & 165. & 0.014 & 170416. & 294.9 & -1.4 & 1.00 \\
\hline 1.94 & 165. & 0.014 & 170470. & 294.9 & -1.4 & 1.00 \\
\hline 2.13 & 181. & 0.012 & 170928. & 294.9 & -1.4 & 1.00 \\
\hline 2.13 & 181. & 0.012 & 170986. & 294.9 & -1.4 & 1.00 \\
\hline 2.13 & 181. & 0.012 & 171035. & 294.9 & -1.4 & 1.00 \\
\hline 2.13 & 181. & 0.012 & 171087. & 294.9 & -1.4 & 1.00 \\
\hline 2.13 & 181. & 0.012 & 171142. & 294.9 & -1.4 & 1.00 \\
\hline 2.13 & 181. & 0.012 & 171194. & 294.9 & -1.4 & 1.00 \\
\hline 2.13 & 181. & 0.012 & 171235. & 294.9 & -1.4 & 1.00 \\
\hline 2.13 & 181. & 0.012 & 171288. & 294.9 & -1.4 & 1.00 \\
\hline 2.13 & 181. & 0.012 & 171334. & 294.9 & -1.3 & 1.00 \\
\hline 2.13 & 181. & 0.012 & 171389. & 294.9 & -1.3 & 1.00 \\
\hline 2.13 & 181. & 0.012 & 171429. & 294.9 & -1.3 & 1.00 \\
\hline 2.13 & 181. & 0.012 & 171476. & 294.9 & -1.3 & 1.00 \\
\hline 2.13 & 181. & 0.012 & 171520. & 294.9 & -1.3 & 1.00 \\
\hline 1.57 & 134. & 0.027 & 197155. & 294.2 & -0.7 & 1.00 \\
\hline 1.57 & 134. & 0.027 & 197198. & 294.2 & -0.7 & 1.00 \\
\hline 1.57 & 134. & 0.027 & 197247. & 294.3 & -0.7 & 1.00 \\
\hline 1.57 & 134. & 0.027 & 197290. & 294.3 & -0.7 & 1.00 \\
\hline 1.57 & 134. & 0.027 & 197343. & 294.3 & -0.8 & 1.00 \\
\hline 1.58 & 134. & 0.004 & 197498. & 294.4 & -0.8 & 1.00 \\
\hline 1.58 & 134. & 0.004 & 197541. & 294.4 & -0.8 & 1.00 \\
\hline 1.58 & 134. & 0.004 & 197581. & 294.4 & -0.8 & 1.00 \\
\hline
\end{tabular}


Table H.2.7 Diesel contamination on oxidized copper surface for $\operatorname{Re}=0$ and $x_{\mathrm{b}}=0.3 \%$ (file:trv0con2.tb2)

\begin{tabular}{|c|c|c|c|c|c|c|c|}
\hline $\begin{array}{c}l_{\mathrm{e}} \\
(\mu \mathrm{m})\end{array}$ & $\begin{array}{c}\Gamma \\
\left(\mathrm{kg} / \mathrm{m}^{2}\right) \\
\mathrm{X} 10^{5} \\
\end{array}$ & $\begin{array}{l}x_{\mathrm{b}} \\
(\%) \\
\end{array}$ & $\begin{array}{c}\text { Exposure } \\
\text { Time (s) }\end{array}$ & $\mathrm{Re}$ & 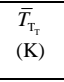 & $\begin{array}{c}T_{\mathrm{b}}-\bar{T}_{\mathrm{T}_{\mathrm{T}}} \\
(\mathrm{K})\end{array}$ & $\frac{F_{T_{\mathrm{b}}}}{F_{T_{\mathrm{T}}}}$ \\
\hline 0.61 & 52. & 0.339 & 2476. & 0. & 295.4 & -1.7 & 1.00 \\
\hline 0.61 & 52. & 0.339 & 2532. & 0. & 295.4 & -1.7 & 1.00 \\
\hline 0.61 & 52. & 0.339 & 2592. & 0. & 295.4 & -1.7 & 1.00 \\
\hline 0.61 & 52. & 0.339 & 2647. & 0. & 295.3 & -1.6 & 1.00 \\
\hline 0.61 & 52. & 0.339 & 2702. & 0. & 295.3 & -1.6 & 1.00 \\
\hline 0.61 & 52. & 0.339 & 2763. & 0. & 295.3 & -1.6 & 1.00 \\
\hline 0.61 & 52. & 0.339 & 2823. & 0. & 295.3 & -1.6 & 1.00 \\
\hline 0.61 & 52. & 0.339 & 2880. & 0. & 295.3 & -1.6 & 1.00 \\
\hline 0.61 & 52. & 0.339 & 2939. & 0. & 295.3 & -1.6 & 1.00 \\
\hline 0.61 & 52. & 0.339 & 3000 . & 0. & 295.3 & -1.6 & 1.00 \\
\hline 0.61 & 52. & 0.339 & 3056. & 0. & 295.3 & -1.6 & 1.00 \\
\hline 0.61 & 52. & 0.339 & 3118. & 0. & 295.3 & -1.6 & 1.00 \\
\hline 0.61 & 52. & 0.339 & 3178. & 0. & 295.2 & -1.6 & 1.00 \\
\hline 1.78 & 151. & 0.300 & 406841. & 0. & 294.6 & -0.9 & 1.00 \\
\hline 1.78 & 151. & 0.300 & 406899. & 0. & 294.6 & -0.9 & 1.00 \\
\hline 1.78 & 151. & 0.300 & 406954. & 0. & 294.6 & -0.9 & 1.00 \\
\hline 1.78 & 151. & 0.300 & 407010. & 0. & 294.6 & -0.9 & 1.00 \\
\hline 1.78 & 151. & 0.300 & 407065 . & 0. & 294.6 & -0.9 & 1.00 \\
\hline 1.78 & 151. & 0.300 & 407122. & 0. & 294.6 & -0.9 & 1.00 \\
\hline 1.78 & 151. & 0.300 & 407178. & 0. & 294.6 & -0.9 & 1.00 \\
\hline 1.78 & 151. & 0.300 & 407234. & 0. & 294.6 & -0.9 & 1.00 \\
\hline 1.78 & 151. & 0.300 & 407289. & 0. & 294.6 & -0.9 & 1.00 \\
\hline 1.78 & 151. & 0.300 & 407354. & 0. & 294.6 & -0.9 & 1.00 \\
\hline 1.78 & 151. & 0.300 & 407410 . & 0 . & 294.6 & -0.9 & 1.00 \\
\hline 1.78 & 151. & 0.300 & 407466. & 0. & 294.6 & -0.9 & 1.00 \\
\hline 1.78 & 151. & 0.300 & 407521. & 0. & 294.6 & -0.9 & 1.00 \\
\hline 2.67 & 226. & 0.283 & 410253. & 0. & 294.7 & -1.0 & 1.00 \\
\hline 2.67 & 226. & 0.283 & 410306. & 0. & 294.7 & -1.0 & 1.00 \\
\hline 2.67 & 226. & 0.283 & 410359. & 0. & 294.7 & -1.0 & 1.00 \\
\hline 2.67 & 226. & 0.283 & 410412. & 0. & 294.7 & -1.0 & 1.00 \\
\hline 2.67 & 226. & 0.283 & 410468. & 0. & 294.7 & -1.0 & 1.00 \\
\hline 2.67 & 226. & 0.283 & 410522. & 0. & 294.7 & -1.0 & 1.00 \\
\hline 2.67 & 226. & 0.283 & 410577. & 0. & 294.7 & -1.0 & 1.00 \\
\hline 2.67 & 226. & 0.283 & 410632. & 0. & 294.7 & -1.0 & 1.00 \\
\hline 2.67 & 226. & 0.283 & 410685. & 0. & 294.7 & -1.0 & 1.00 \\
\hline 2.67 & 226. & 0.283 & 410739. & 0. & 294.7 & -1.0 & 1.00 \\
\hline 2.67 & 226. & 0.283 & 410798. & 0. & 294.7 & -1.0 & 1.00 \\
\hline 2.67 & 226. & 0.283 & 410853. & 0. & 294.7 & -1.0 & 1.00 \\
\hline 2.67 & 226. & 0.283 & 410915. & 0. & 294.7 & -1.0 & 1.00 \\
\hline 3.17 & 269. & 0.280 & 414268. & 0. & 294.9 & -1.1 & 1.00 \\
\hline 3.17 & 269. & 0.280 & 414324. & 0. & 294.8 & -1.1 & 1.00 \\
\hline 3.17 & 269. & 0.280 & 414380. & 0. & 294.8 & -1.1 & 1.00 \\
\hline 3.17 & 269. & 0.280 & 414435. & 0. & 294.8 & -1.1 & 1.00 \\
\hline 3.17 & 269. & 0.280 & 414494. & 0. & 294.8 & -1.1 & 1.00 \\
\hline 3.17 & 269. & 0.280 & 414545. & 0. & 294.8 & -1.1 & 1.00 \\
\hline 3.17 & 269. & 0.280 & 414605. & 0. & 294.8 & -1.1 & 1.00 \\
\hline 3.17 & 269. & 0.280 & 414662. & 0. & 294.8 & -1.1 & 1.00 \\
\hline 3.17 & 269. & 0.280 & 414717. & 0. & 294.8 & -1.1 & 1.00 \\
\hline 3.17 & 269. & 0.280 & 414775. & 0. & 294.8 & -1.1 & 1.00 \\
\hline 3.17 & 269. & 0.280 & 414834. & 0. & 294.8 & -1.1 & 1.00 \\
\hline 3.17 & 269. & 0.280 & 414889. & 0. & 294.8 & -1.1 & 1.00 \\
\hline 3.17 & 269. & 0.280 & 414947. & 0. & 294.8 & -1.1 & 1.00 \\
\hline 3.86 & 327. & 0.272 & 418388. & 0. & 294.9 & -1.2 & 1.00 \\
\hline 3.86 & 327. & 0.272 & 418457. & 0. & 294.9 & -1.2 & 1.00 \\
\hline 3.86 & 327. & 0.272 & 418510 . & 0. & 294.9 & -1.2 & 1.00 \\
\hline 3.86 & 327. & 0.272 & 418565. & 0. & 294.9 & -1.2 & 1.00 \\
\hline 3.86 & 327. & 0.272 & 418620. & 0. & 294.9 & -1.2 & 1.00 \\
\hline 3.86 & 327. & 0.272 & 418674. & 0. & 294.9 & -1.2 & 1.00 \\
\hline 3.86 & 327. & 0.272 & 418730. & 0. & 294.9 & -1.2 & 1.00 \\
\hline 3.86 & 327. & 0.272 & 418783. & 0. & 294.9 & -1.2 & 1.00 \\
\hline 3.86 & 327. & 0.272 & 418838. & 0. & 294.9 & -1.2 & 1.00 \\
\hline 3.86 & 327. & 0.272 & 418892 . & 0. & 294.9 & -1.2 & 1.00 \\
\hline 3.86 & 327. & 0.272 & 418945. & 0. & 294.9 & -1.2 & 1.00 \\
\hline 3.86 & 327. & 0.272 & 419001. & 0. & 294.9 & -1.2 & 1.00 \\
\hline 3.86 & 327. & 0.272 & 419056. & 0. & 294.9 & $\begin{array}{l}-1.2 \\
\end{array}$ & 1.00 \\
\hline 4.30 & 364. & 0.259 & 421712. & 0. & 294.9 & -1.2 & 1.00 \\
\hline 4.30 & 364. & 0.259 & 421767. & 0. & 294.9 & -1.2 & 1.00 \\
\hline 4.30 & 364. & 0.259 & 421821. & 0. & 294.9 & $\begin{array}{l}-1.2 \\
\end{array}$ & 1.00 \\
\hline 4.30 & 364. & 0.259 & 421888. & 0. & 294.9 & $\begin{array}{l}-1.2 \\
\end{array}$ & 1.00 \\
\hline 4.30 & 364. & 0.259 & 421940 . & 0. & 294.9 & -1.2 & 1.00 \\
\hline 4.30 & 364. & 0.259 & 421991. & 0. & 294.9 & $\begin{array}{l}-1.2 \\
\end{array}$ & 1.00 \\
\hline 4.30 & 364. & 0.259 & 422044. & 0. & 294.9 & -1.2 & 1.00 \\
\hline 4.30 & 364. & 0.259 & 422101. & 0. & 294.9 & -1.2 & 1.00 \\
\hline 4.30 & 364. & 0.259 & 422155. & 0. & 294.9 & $\begin{array}{l}-1.2 \\
\end{array}$ & 1.00 \\
\hline 4.30 & 364. & 0.259 & 422206. & 0. & 294.9 & $\begin{array}{l}-1.2 \\
\end{array}$ & 1.00 \\
\hline 4.30 & 364. & 0.259 & 422261. & 0. & 294.9 & -1.2 & 1.00 \\
\hline 4.30 & 364. & 0.259 & 422317. & 0. & 294.9 & -1.2 & 1.00 \\
\hline
\end{tabular}

\begin{tabular}{|c|c|c|c|c|c|c|c|}
\hline 4.30 & 364. & 0.259 & 422372. & 0. & 294.9 & -1.2 & 1.00 \\
\hline 4.45 & 377. & 0.263 & 425568. & 0 . & 294.9 & -1.1 & 1.00 \\
\hline 4.45 & 377. & 0.263 & 425621. & 0. & 294.9 & -1.2 & 1.00 \\
\hline 4.45 & 377. & 0.263 & 425677. & 0. & 294.9 & -1.2 & 1.00 \\
\hline 4.45 & 377. & 0.263 & 425735. & 0. & 294.9 & -1.1 & 1.00 \\
\hline 4.45 & 377. & 0.263 & 425790. & 0. & 294.9 & -1.2 & 1.00 \\
\hline 4.45 & 377. & 0.263 & 425843. & 0. & 294.9 & -1.2 & 1.00 \\
\hline 4.45 & 377. & 0.263 & 425899. & 0. & 294.9 & -1.1 & 1.00 \\
\hline 4.45 & 377. & 0.263 & 425954. & 0. & 294.9 & -1.1 & 1.00 \\
\hline 4.45 & 377. & 0.263 & 426011. & 0. & 294.9 & -1.1 & 1.00 \\
\hline 4.45 & 377. & 0.263 & 426070. & 0. & 294.9 & -1.2 & 1.00 \\
\hline 4.45 & 377. & 0.263 & 426126. & 0. & 294.9 & -1.2 & 1.00 \\
\hline 4.45 & 377. & 0.263 & 426179. & 0. & 294.9 & -1.2 & 1.00 \\
\hline 4.45 & 377. & 0.263 & 426237. & 0. & 294.9 & -1.2 & 1.00 \\
\hline 3.77 & 319. & 0.290 & 429254. & 0. & 294.8 & -1.1 & 1.00 \\
\hline 3.77 & 319. & 0.290 & 429314. & 0. & 294.8 & -1.1 & 1.00 \\
\hline 3.77 & 319. & 0.290 & 429368. & 0. & 294.8 & -1.1 & 1.00 \\
\hline 3.77 & 319. & 0.290 & 429432. & 0. & 294.8 & -1.1 & 1.00 \\
\hline 3.77 & 319. & 0.290 & 429509. & 0. & 294.8 & -1.1 & 1.00 \\
\hline 3.77 & 319. & 0.290 & 429571. & 0. & 294.8 & -1.1 & 1.00 \\
\hline 3.77 & 319. & 0.290 & 429625. & 0. & 294.8 & -1.1 & 1.00 \\
\hline 3.77 & 319. & 0.290 & 429684. & 0. & 294.8 & -1.1 & 1.00 \\
\hline 3.77 & 319. & 0.290 & 429735. & 0. & 294.8 & -1.1 & 1.00 \\
\hline 3.77 & 319. & 0.290 & 429789. & 0. & 294.8 & -1.1 & 1.00 \\
\hline 3.77 & 319. & 0.290 & 429844. & 0. & 294.8 & -1.1 & 1.00 \\
\hline 3.77 & 319. & 0.290 & 429900. & 0. & 294.8 & -1.1 & 1.00 \\
\hline 3.77 & 319. & 0.290 & 429954. & 0. & 294.8 & -1.1 & 1.00 \\
\hline 3.72 & 316. & 0.267 & 432844. & 0. & 294.8 & -1.1 & 1.00 \\
\hline 3.72 & 316. & 0.267 & 432904. & 0. & 294.7 & -1.0 & 1.00 \\
\hline 3.72 & 316. & 0.267 & 432958. & 0. & 294.8 & -1.1 & 1.00 \\
\hline 3.72 & 316. & 0.267 & 433013. & 0. & 294.7 & -1.1 & 1.00 \\
\hline 3.72 & 316. & 0.267 & 433077. & 0. & 294.7 & -1.1 & 1.00 \\
\hline 3.72 & 316. & 0.267 & 433130. & 0. & 294.7 & -1.1 & 1.00 \\
\hline 3.72 & 316. & 0.267 & 433190. & 0. & 294.7 & -1.0 & 1.00 \\
\hline 3.72 & 316. & 0.267 & 433242. & 0. & 294.7 & -1.1 & 1.00 \\
\hline 3.72 & 316. & 0.267 & 433301. & 0. & 294.7 & -1.1 & 1.00 \\
\hline 3.72 & 316. & 0.267 & 433361. & 0. & 294.7 & -1.0 & 1.00 \\
\hline 3.72 & 316. & 0.267 & 433416. & 0. & 294.7 & -1.1 & 1.00 \\
\hline 3.72 & 316. & 0.267 & 433472. & 0. & 294.7 & -1.0 & 1.00 \\
\hline 3.72 & 316. & 0.267 & 433527. & 0. & 294.7 & -1.0 & 1.00 \\
\hline 3.41 & 289. & 0.275 & 494999. & 0. & 294.5 & -0.8 & 1.00 \\
\hline 3.41 & 289. & 0.275 & 495055. & 0. & 294.5 & -0.8 & 1.00 \\
\hline 3.41 & 289. & 0.275 & 495113. & 0. & 294.5 & -0.8 & 1.00 \\
\hline 3.41 & 289. & 0.275 & 495188. & 0. & 294.5 & -0.8 & 1.00 \\
\hline 3.41 & 289. & 0.275 & 495257. & 0. & 294.5 & -0.8 & 1.00 \\
\hline 3.41 & 289. & 0.275 & 495315. & 0. & 294.5 & -0.8 & 1.00 \\
\hline 3.41 & 289. & 0.275 & 495369. & 0. & 294.5 & -0.8 & 1.00 \\
\hline 3.41 & 289. & 0.275 & 495611. & 0. & 294.5 & -0.8 & 1.00 \\
\hline 3.41 & 289. & 0.275 & 495673. & 0. & 294.5 & -0.8 & 1.00 \\
\hline 3.41 & 289. & 0.275 & 495728. & 0. & 294.5 & -0.8 & 1.00 \\
\hline 3.41 & 289. & 0.275 & 495786. & 0. & 294.5 & -0.8 & 1.00 \\
\hline 3.41 & 289. & 0.275 & 495839. & 0. & 294.5 & -0.8 & 1.00 \\
\hline 3.41 & 289. & 0.275 & 495902. & 0. & 294.5 & -0.8 & 1.00 \\
\hline 4.23 & 358. & 0.267 & 498198. & 0. & 294.6 & -0.9 & 1.00 \\
\hline 4.23 & 358. & 0.267 & 498282. & 0. & 294.6 & -0.9 & 1.00 \\
\hline 4.23 & 358. & 0.267 & 498336. & 0. & 294.6 & -0.9 & 1.00 \\
\hline 4.23 & 358. & 0.267 & 498412. & 0. & 294.6 & -0.9 & 1.00 \\
\hline 4.23 & 358. & 0.267 & 498495. & 0. & 294.6 & -0.9 & 1.00 \\
\hline 4.23 & 358. & 0.267 & 498579. & 0. & 294.6 & -0.9 & 1.00 \\
\hline 4.23 & 358. & 0.267 & 498644. & 0. & 294.6 & -0.9 & 1.00 \\
\hline 4.23 & 358. & 0.267 & 498708. & 0. & 294.6 & -0.9 & 1.00 \\
\hline 4.23 & 358. & 0.267 & 498768. & 0. & 294.6 & -0.9 & 1.00 \\
\hline 4.23 & 358. & 0.267 & 498826. & 0. & 294.6 & -0.9 & 1.00 \\
\hline 4.23 & 358. & 0.267 & 498883. & 0. & 294.6 & -0.9 & 1.00 \\
\hline 4.23 & 358. & 0.267 & 498955. & 0. & 294.6 & -0.9 & 1.00 \\
\hline 4.23 & 358. & 0.267 & 499011. & 0. & 294.6 & -0.9 & 1.00 \\
\hline 5.72 & 485. & 0.285 & 506417. & 0. & 294.6 & -0.9 & 1.00 \\
\hline 5.72 & 485. & 0.285 & 506473. & 0. & 294.6 & -0.9 & 1.00 \\
\hline 5.72 & 485. & 0.285 & 506525. & 0. & 294.6 & -0.9 & 1.00 \\
\hline 5.72 & 485. & 0.285 & 506579. & 0. & 294.6 & -0.9 & 1.00 \\
\hline 5.72 & 485. & 0.285 & 506636. & 0. & 294.6 & -0.9 & 1.00 \\
\hline 5.72 & 485. & 0.285 & 506692. & 0. & 294.6 & -0.9 & 1.00 \\
\hline 5.72 & 485. & 0.285 & 506755. & 0. & 294.6 & -0.9 & 1.00 \\
\hline 5.72 & 485. & 0.285 & 506811. & 0. & 294.6 & -0.9 & 1.00 \\
\hline 5.72 & 485. & 0.285 & 506866. & 0. & 294.6 & -0.9 & 1.00 \\
\hline 5.72 & 485. & 0.285 & 506921. & 0. & 294.6 & -0.9 & 1.00 \\
\hline 5.72 & 485. & 0.285 & 506977. & 0. & 294.6 & -0.9 & 1.00 \\
\hline 5.72 & 485. & 0.285 & 507031. & 0. & 294.6 & -0.9 & 1.00 \\
\hline 5.72 & 485. & 0.285 & 507090. & 0. & 294.6 & -0.9 & 1.00 \\
\hline 4.54 & 384. & 0.260 & 82346. & 0. & 295.6 & 0.2 & 1.00 \\
\hline
\end{tabular}


Table H.2.8 Diesel contamination on oxidized copper surface for $\mathrm{Re}=2000$ and $x_{\mathrm{b}}=0.3 \%$ (file:trv15con2.tb2)

\begin{tabular}{|c|c|c|c|c|c|c|c|}
\hline $\begin{array}{c}l_{\mathrm{e}} \\
(\mu \mathrm{m})\end{array}$ & $\begin{array}{c}\Gamma \\
\left(\mathrm{kg} / \mathrm{m}^{2}\right) \\
\mathrm{X} 10^{5}\end{array}$ & $\begin{array}{l}x_{\mathrm{b}} \\
(\%)\end{array}$ & $\begin{array}{l}\text { Exposure } \\
\text { Time (s) }\end{array}$ & $\operatorname{Re}$ & $\begin{array}{l}\bar{T}_{\mathrm{T}_{\mathrm{T}}} \\
(\mathrm{K})\end{array}$ & $\begin{array}{c}T_{\mathrm{b}}-\bar{T}_{\mathrm{T}_{\mathrm{T}}} \\
(\mathrm{K})\end{array}$ & $\frac{F_{T_{b}}}{F_{T_{\mathrm{T}}}}$ \\
\hline-0.77 & -65 & 0.335 & 613. & 1909. & 294.5 & $\begin{array}{l}-0.8 \\
\end{array}$ & 1.00 \\
\hline-0.77 & -65. & 0.335 & 749. & 1961. & 294.5 & -0.8 & 1.00 \\
\hline-0.77 & -65. & 0.335 & 792. & 1984. & 294.5 & -0.8 & 1.00 \\
\hline-0.77 & -65. & 0.335 & 837. & 1903. & 294.5 & -0.8 & 1.00 \\
\hline-0.77 & -65. & 0.335 & 881. & 1914. & 294.5 & -0.8 & 1.00 \\
\hline-0.77 & -65. & 0.335 & 922. & 1982. & 294.5 & -0.8 & 1.00 \\
\hline-0.77 & -65. & 0.335 & 966. & 2003. & 294.5 & -0.8 & 1.00 \\
\hline-0.77 & -65. & 0.335 & 1008. & 1967. & 294.5 & $\begin{array}{l}-0.8 \\
\end{array}$ & 1.00 \\
\hline-0.77 & -65. & 0.335 & 1054. & 1934. & 294.4 & -0.8 & 1.00 \\
\hline-0.77 & -65. & 0.335 & 1097. & 1983. & 294.4 & -0.8 & 1.00 \\
\hline-0.77 & -65. & 0.335 & 1138. & 1865. & 294.4 & -0.8 & 1.00 \\
\hline-0.77 & -65. & 0.335 & 1181. & 2000. & 294.4 & -0.7 & 1.00 \\
\hline-0.77 & -65. & 0.335 & 1226. & 1945. & 294.4 & -0.7 & 1.00 \\
\hline-0.07 & -6. & 0.310 & 1374. & 1958. & 294.4 & -0.6 & 1.00 \\
\hline-0.07 & -6. & 0.310 & 1422. & 1888. & 294.3 & -0.7 & 1.00 \\
\hline-0.07 & -6. & 0.310 & 1468. & 1975. & 294.3 & -0.6 & 1.00 \\
\hline-0.07 & -6. & 0.310 & 1513. & 1982. & 294.3 & -0.6 & 1.00 \\
\hline-0.07 & -6. & 0.310 & 1557. & 1877. & 294.3 & -0.6 & 1.00 \\
\hline-0.07 & -6. & 0.310 & 1606. & 1898. & 294.3 & -0.6 & 1.00 \\
\hline-0.07 & -6. & 0.310 & 1652. & 1965. & 294.2 & -0.6 & 1.00 \\
\hline-0.07 & -6. & 0.310 & 1694. & 1954. & 294.2 & -0.5 & 1.00 \\
\hline-0.07 & -6. & 0.310 & 1739. & 1911. & 294.2 & -0.5 & 1.00 \\
\hline-0.07 & -6. & 0.310 & 1786. & 1914. & 294.1 & -0.5 & 1.00 \\
\hline-0.07 & -6. & 0.310 & 1831. & 1836. & 294.1 & -0.5 & 1.00 \\
\hline-0.07 & -6. & 0.310 & 1875. & 1953. & 294.1 & -0.4 & 1.00 \\
\hline-0.07 & -6. & 0.310 & 1919. & 1861. & 294.1 & -0.4 & 1.00 \\
\hline 9.14 & 772. & 0.673 & 5348. & 1811. & 293.2 & 0.4 & 1.00 \\
\hline 9.14 & 772. & 0.673 & 5391. & 1811. & 293.2 & 0.4 & 1.00 \\
\hline 9.14 & 772. & 0.673 & 5440. & 1850. & 293.3 & 0.4 & 1.00 \\
\hline 9.14 & 772. & 0.673 & 5485. & 1723. & 293.3 & 0.4 & 1.00 \\
\hline 9.14 & 772. & 0.673 & 5528. & 1796. & 293.3 & 0.4 & 1.00 \\
\hline 9.14 & 772. & 0.673 & 5573. & 1702. & 293.3 & 0.4 & 1.00 \\
\hline 9.14 & 772. & 0.673 & 5620. & 1788. & 293.3 & 0.3 & 1.00 \\
\hline 9.14 & 772. & 0.673 & 5667. & 1817. & 293.3 & 0.3 & 1.00 \\
\hline 9.14 & 771. & 0.673 & 5712. & 1749. & 293.4 & 0.3 & 1.00 \\
\hline 9.14 & 771. & 0.673 & 5756. & 1713. & 293.4 & 0.3 & 1.00 \\
\hline 9.14 & 771. & 0.673 & 5804. & 1748. & 293.4 & 0.3 & 1.00 \\
\hline 9.14 & 771. & 0.673 & 5855. & 1784. & 293.4 & 0.2 & 1.00 \\
\hline 9.14 & 771. & 0.673 & 5910. & 1691. & 293.4 & 0.2 & 1.00 \\
\hline 15.37 & 1298. & 0.588 & 11545. & 1871. & 293.7 & 0.0 & 1.00 \\
\hline 15.37 & 1298. & 0.588 & 11590. & 1866. & 293.7 & 0.0 & 1.00 \\
\hline 15.37 & 1298. & 0.588 & 11635. & 1864. & 293.7 & -0.1 & 1.00 \\
\hline 15.37 & 1298. & 0.588 & 11678. & 1848. & 293.7 & -0.1 & 1.00 \\
\hline 15.37 & 1298. & 0.588 & 11723. & 1866. & 293.8 & -0.1 & 1.00 \\
\hline 15.37 & 1298. & 0.588 & 11769. & 1875. & 293.8 & -0.1 & 1.00 \\
\hline 15.37 & 1298. & 0.588 & 11820. & 1897. & 293.8 & -0.1 & 1.00 \\
\hline 15.37 & 1298. & 0.588 & 11885. & 1873. & 293.8 & $\begin{array}{l}-0.2 \\
\end{array}$ & 1.00 \\
\hline 15.37 & 1298. & 0.588 & 11937. & 1911. & 293.8 & $\begin{array}{l}-0.2 \\
\end{array}$ & 1.00 \\
\hline 15.37 & 1298. & 0.588 & 11982. & 1881. & 293.9 & -0.2 & 1.00 \\
\hline 15.37 & 1298. & 0.588 & 12028. & 1803. & 293.9 & -0.2 & 1.00 \\
\hline 15.37 & 1298. & 0.588 & 12073. & 1789. & 293.9 & -0.2 & 1.00 \\
\hline 15.37 & 1298. & 0.588 & 12118. & 1852. & 293.9 & -0.2 & 1.00 \\
\hline 13.56 & 1149. & 0.323 & 12289. & 1923. & 293.9 & $\begin{array}{l}-0.3 \\
\end{array}$ & 1.00 \\
\hline 13.56 & 1149. & 0.323 & 12336. & 1804. & 293.9 & -0.3 & 1.00 \\
\hline 13.56 & 1149. & 0.323 & 12382. & 1873. & 293.9 & -0.3 & 1.00 \\
\hline 13.56 & 1149. & 0.323 & 12433. & 1873. & 293.9 & -0.3 & 1.00 \\
\hline 13.56 & 1149. & 0.323 & 12476. & 1827. & 293.9 & -0.3 & 1.00 \\
\hline 13.56 & 1149. & 0.323 & 12523. & 1887. & 293.9 & -0.3 & 1.00 \\
\hline 13.56 & 1149. & 0.323 & 12570. & 1829. & 293.9 & $\begin{array}{l}-0.3 \\
\end{array}$ & 1.00 \\
\hline 13.56 & 1149. & 0.323 & 12616. & 1889. & 293.9 & -0.3 & 1.00 \\
\hline 13.56 & 1149. & 0.323 & 12664. & 1803. & 293.9 & -0.3 & 1.00 \\
\hline 13.56 & 1149. & 0.323 & 12711. & 1816. & 293.9 & $\begin{array}{l}-0.3 \\
\end{array}$ & 1.00 \\
\hline 13.56 & 1149. & 0.323 & 12755. & 1897. & 293.9 & $\begin{array}{l}-0.2 \\
\end{array}$ & 1.00 \\
\hline 13.56 & 1149. & 0.323 & 12803. & 1839. & 293.9 & \begin{tabular}{|l|}
-0.3 \\
\end{tabular} & 1.00 \\
\hline 13.56 & 1149. & 0.323 & 12851. & 1820. & 293.9 & -0.2 & 1.00 \\
\hline 12.76 & 1081. & 0.359 & 13002. & 1819. & 293.9 & $\begin{array}{l}-0.2 \\
\end{array}$ & 1.00 \\
\hline 12.76 & 1081. & 0.359 & 13051. & 1896. & 293.9 & -0.2 & 1.00 \\
\hline 12.76 & 1081. & 0.359 & 13104. & 1907. & 293.8 & -0.2 & 1.00 \\
\hline 12.76 & 1081. & 0.359 & 13151. & 1801. & 293.8 & $\begin{array}{l}-0.2 \\
\end{array}$ & 1.00 \\
\hline 12.76 & 1081. & 0.359 & 13210 . & 1897. & 293.8 & $\begin{array}{l}-0.1 \\
\end{array}$ & 1.00 \\
\hline 12.76 & 1081. & 0.359 & 13262. & 1865. & 293.8 & -0.1 & 1.00 \\
\hline 12.76 & 1081. & 0.359 & 13308. & 1888. & 293.8 & $\begin{array}{l}-0.1 \\
\end{array}$ & 1.00 \\
\hline 12.76 & 1081. & 0.359 & 13363. & 1824. & 293.7 & -0.1 & 1.00 \\
\hline 12.76 & 1081. & 0.359 & 13408. & 1797. & 293.7 & -0.1 & 1.00 \\
\hline 12.76 & 1081. & 0.359 & 13451. & 1797. & 293.7 & 0.0 & 1.00 \\
\hline
\end{tabular}

\begin{tabular}{|c|c|c|c|c|c|c|c|}
\hline 12.76 & 1081. & 0.359 & 13494. & 1874. & 293.7 & 0.0 & 1.00 \\
\hline 12.76 & 1081. & 0.359 & 13537. & 1817. & 293.7 & 0.0 & 1.00 \\
\hline 12.76 & 1081. & 0.359 & 13588. & 1846. & 293.6 & 0.0 & 1.00 \\
\hline 18.36 & 1558. & 0.216 & 73355. & 1865. & 293.7 & -0.1 & 1.00 \\
\hline 18.36 & 1558. & 0.216 & 73397. & 1847. & 293.8 & -0.1 & 1.00 \\
\hline 18.36 & 1558. & 0.216 & 73442. & 1836. & 293.8 & -0.1 & 1.00 \\
\hline 18.36 & 1558. & 0.216 & 73485. & 1884. & 293.8 & -0.1 & 1.00 \\
\hline 18.36 & 1558. & 0.216 & 73528. & 1809. & 293.8 & -0.2 & 1.00 \\
\hline 18.36 & 1558. & 0.216 & 73573. & 1894. & 293.8 & -0.2 & 1.00 \\
\hline 18.36 & 1558. & 0.216 & 73619. & 1850. & 293.9 & -0.2 & 1.00 \\
\hline 18.36 & 1558. & 0.216 & 73662. & 1842. & 293.9 & -0.2 & 1.00 \\
\hline 18.36 & 1558. & 0.216 & 73704. & 1782. & 293.9 & -0.2 & 1.00 \\
\hline 18.36 & 1558. & 0.216 & 73747. & 1872. & 293.9 & -0.2 & 1.00 \\
\hline 18.36 & 1558. & 0.216 & 73794. & 1893. & 293.9 & -0.3 & 1.00 \\
\hline 18.36 & 1558. & 0.216 & 73842. & 1803. & 293.9 & -0.3 & 1.00 \\
\hline 18.36 & 1558. & 0.216 & 73894. & 1880 . & 293.9 & -0.3 & 1.00 \\
\hline 18.48 & 1569. & 0.189 & 77085. & 1886. & 293.3 & 0.4 & 1.00 \\
\hline 18.48 & 1569. & 0.189 & 77131. & 1847. & 293.3 & 0.4 & 1.00 \\
\hline 18.48 & 1569. & 0.189 & 77174. & 1780. & 293.3 & 0.4 & 1.00 \\
\hline 18.48 & 1569. & 0.189 & 77217. & 1834. & 293.3 & 0.4 & 1.00 \\
\hline 18.48 & 1569. & 0.189 & 77258. & 1780. & 293.3 & 0.4 & 1.00 \\
\hline 18.48 & 1569. & 0.189 & 77301. & 1861. & 293.3 & 0.4 & 1.00 \\
\hline 18.48 & 1569. & 0.189 & 77344. & 1837. & 293.3 & 0.4 & 1.00 \\
\hline 18.48 & 1569. & 0.189 & 77387. & 1782. & 293.3 & 0.3 & 1.00 \\
\hline 18.48 & 1569. & 0.189 & 77431. & 1806. & 293.3 & 0.3 & 1.00 \\
\hline 18.48 & 1569. & 0.189 & 77476 . & 1857. & 293.3 & 0.3 & 1.00 \\
\hline 18.48 & 1569. & 0.189 & 77518. & 1849. & 293.4 & 0.3 & 1.00 \\
\hline 18.48 & 1569. & 0.189 & 77562. & 1857. & 293.4 & 0.3 & 1.00 \\
\hline 18.48 & 1569. & 0.189 & 77605. & 1842. & 293.4 & 0.3 & 1.00 \\
\hline 18.26 & 1550. & 0.173 & 80433. & 1841. & 293.6 & 0.0 & 1.00 \\
\hline 18.26 & 1550. & 0.173 & 80475. & 1812. & 293.6 & 0.1 & 1.00 \\
\hline 18.26 & 1550. & 0.173 & 80517. & 1889. & 293.6 & 0.1 & 1.00 \\
\hline 18.26 & 1550. & 0.173 & 80561. & 1817. & 293.6 & 0.1 & 1.00 \\
\hline 18.26 & 1550. & 0.173 & 80603. & 1885. & 293.6 & 0.1 & 1.00 \\
\hline 18.26 & 1550. & 0.173 & 80650 . & 1878. & 293.5 & 0.1 & 1.00 \\
\hline 18.26 & 1550. & 0.173 & 80695. & 1864. & 293.5 & 0.1 & 1.00 \\
\hline 18.26 & 1550. & 0.173 & 80743. & 1861. & 293.5 & 0.2 & 1.00 \\
\hline 18.26 & 1550. & 0.173 & 80788. & 1826. & 293.4 & 0.2 & 1.00 \\
\hline 18.26 & 1550 . & 0.173 & 80833. & 1834. & 293.4 & 0.2 & 1.00 \\
\hline 18.26 & 1550. & 0.173 & 80883. & 1825. & 293.4 & 0.3 & 1.00 \\
\hline 18.26 & 1550. & 0.173 & 80928. & 1818. & 293.4 & 0.3 & 1.00 \\
\hline 18.26 & 1550. & 0.173 & 80973. & 1819. & 293.4 & 0.3 & 1.00 \\
\hline 17.83 & 1513. & 0.210 & 81131. & 1791. & 293.3 & 0.4 & 1.00 \\
\hline 17.83 & 1513. & 0.210 & 81176. & 1873. & 293.3 & 0.4 & 1.00 \\
\hline 17.83 & 1513. & 0.210 & 81219. & 1884. & 293.3 & 0.4 & 1.00 \\
\hline 17.83 & 1513. & 0.210 & 81265. & 1801. & 293.3 & 0.4 & 1.00 \\
\hline 17.83 & 1513. & 0.210 & 81310. & 1781. & 293.3 & 0.4 & 1.00 \\
\hline 17.83 & 1513. & 0.210 & 81354. & 1794. & 293.2 & 0.4 & 1.00 \\
\hline 17.83 & 1513. & 0.210 & 81403. & 1763. & 293.2 & 0.4 & 1.00 \\
\hline 17.83 & 1513. & 0.210 & 81450. & 1845. & 293.2 & 0.4 & 1.00 \\
\hline 17.83 & 1513. & 0.210 & 81494. & 1825. & 293.2 & 0.5 & 1.00 \\
\hline 17.83 & 1513. & 0.210 & 81540. & 1826. & 293.2 & 0.4 & 1.00 \\
\hline 17.83 & 1513. & 0.210 & 81583. & 1881. & 293.2 & 0.5 & 1.00 \\
\hline 17.83 & 1513. & 0.210 & 81629. & 1819. & 293.2 & 0.5 & 1.00 \\
\hline 17.83 & 1513. & 0.210 & 81674. & 1874. & 293.2 & 0.5 & 1.00 \\
\hline 17.97 & 1509. & 1.095 & 84266. & 1875. & 293.9 & -0.3 & 1.00 \\
\hline 17.97 & 1509. & 1.095 & 84307. & 1902. & 293.9 & -0.3 & 1.00 \\
\hline 17.97 & 1509. & 1.095 & 84352. & 1914. & 293.9 & -0.3 & 1.00 \\
\hline 17.97 & 1509. & 1.095 & 84400 . & 1945. & 293.9 & -0.3 & 1.00 \\
\hline 17.97 & 1509. & 1.095 & 84447. & 1917. & 294.0 & -0.3 & 1.00 \\
\hline 17.97 & 1509. & 1.095 & 84491. & 1902. & 293.9 & -0.3 & 1.00 \\
\hline 17.97 & 1509. & 1.095 & 84539. & 1918. & 293.9 & -0.3 & 1.00 \\
\hline 17.97 & 1509. & 1.095 & 84584. & 1960. & 293.9 & -0.3 & 1.00 \\
\hline 17.97 & 1509. & 1.095 & 84629. & 1949. & 293.9 & -0.3 & 1.00 \\
\hline 17.97 & 1509. & 1.095 & 84675. & 1924. & 293.9 & -0.3 & 1.00 \\
\hline 17.97 & 1509. & 1.095 & 84727. & 1856. & 293.9 & -0.3 & 1.00 \\
\hline 17.97 & 1509. & 1.095 & 84772. & 1851. & 293.9 & -0.3 & 1.00 \\
\hline 17.97 & 1509. & 1.095 & 84818. & 1912. & 293.9 & -0.3 & 1.00 \\
\hline 17.00 & 1440. & 0.384 & 91589. & 1906. & 293.5 & 0.2 & 1.00 \\
\hline 17.00 & 1440. & 0.384 & 91636. & 1874. & 293.4 & 0.2 & 1.00 \\
\hline 17.00 & 1440. & 0.384 & 91681. & 1924. & 293.4 & 0.3 & 1.00 \\
\hline 17.00 & 1440. & 0.384 & 91730. & 1960. & 293.4 & 0.3 & 1.00 \\
\hline 17.00 & 1440. & 0.384 & 91781. & 1946. & 293.4 & 0.3 & 1.00 \\
\hline 17.00 & 1440. & 0.384 & 91827. & 1938. & 293.4 & 0.3 & 1.00 \\
\hline 17.00 & 1440. & 0.384 & 91873. & 1894. & 293.3 & 0.3 & 1.00 \\
\hline 17.00 & 1440. & 0.384 & 91918. & 1858. & 293.3 & 0.4 & 1.00 \\
\hline 17.00 & 1440. & 0.384 & 91977. & 1876. & 293.3 & 0.3 & 1.00 \\
\hline 17.00 & 1440. & 0.384 & 92034. & 1819. & 293.3 & 0.4 & 1.00 \\
\hline
\end{tabular}


Table H.2.9 Diesel contamination on oxidized copper surface for $\mathrm{Re}=4000$ and $x_{\mathrm{b}}=0.3 \%$ (file:trv3con2.tb2)

\begin{tabular}{|c|c|c|c|c|c|c|c|}
\hline $\begin{array}{c}l_{\mathrm{e}} \\
(\mu \mathrm{m})\end{array}$ & $\begin{array}{c}\Gamma \\
\left(\mathrm{kg} / \mathrm{m}^{2}\right) \\
\mathrm{X} 10^{5}\end{array}$ & $\begin{array}{l}x_{\mathrm{b}} \\
(\%)\end{array}$ & $\begin{array}{l}\text { Exposure } \\
\text { Time (s) }\end{array}$ & $\mathrm{Re}$ & $\begin{array}{l}\bar{T}_{\mathrm{T}_{\mathrm{T}}} \\
(\mathrm{K})\end{array}$ & $\begin{array}{c}T_{\mathrm{b}}-\bar{T}_{\mathrm{T}_{\mathrm{T}}} \\
(\mathrm{K})\end{array}$ & $\frac{F_{T_{b}}}{F_{T_{\mathrm{T}}}}$ \\
\hline 11.75 & 995. & 0.302 & 64840. & 3944. & 293.9 & -0.2 & 1.00 \\
\hline 11.75 & 995. & 0.302 & 64910. & 4053. & 293.9 & -0.2 & 1.00 \\
\hline 11.75 & 996. & 0.302 & 64953. & 4038. & 293.9 & -0.2 & 1.00 \\
\hline 11.75 & 996. & 0.302 & 65012. & 3912. & 293.8 & -0.2 & 1.00 \\
\hline 11.75 & 996. & 0.302 & 65079. & 3984. & 293.8 & -0.1 & 1.00 \\
\hline 11.75 & 996. & 0.302 & 65125. & 3991. & 293.8 & -0.1 & 1.00 \\
\hline 11.75 & 996. & 0.302 & 65175. & 4016. & 293.8 & -0.1 & 1.00 \\
\hline 11.75 & 996. & 0.302 & 65229. & 4003. & 293.8 & -0.1 & 1.00 \\
\hline 11.75 & 996. & 0.302 & 65299. & 4098. & 293.7 & 0.0 & 1.00 \\
\hline 11.75 & 996. & 0.302 & 65349. & 3970. & 293.7 & 0.0 & 1.00 \\
\hline 11.75 & 996. & 0.302 & 65421. & 3941. & 293.6 & 0.0 & 1.00 \\
\hline 11.75 & 996. & 0.302 & 65505. & 4076. & 293.6 & 0.1 & 1.00 \\
\hline 11.75 & 996. & 0.302 & 65555. & 3885. & 293.5 & 0.1 & 1.00 \\
\hline 12.51 & 1060 . & 0.283 & 68691. & 3973. & 294.0 & -0.3 & 1.00 \\
\hline 12.51 & 1060. & 0.283 & 68759. & 4069. & 293.9 & -0.2 & 1.00 \\
\hline 12.51 & 1060. & 0.283 & 68808 . & 4134. & 293.9 & -0.2 & 1.00 \\
\hline 12.51 & 1060 . & 0.283 & 68854. & 3967. & 293.9 & -0.2 & 1.00 \\
\hline 12.51 & 1060. & 0.283 & 68898. & 3847. & 293.9 & -0.2 & 1.00 \\
\hline 12.51 & 1060 . & 0.283 & 68943. & 4078. & 293.9 & -0.2 & 1.00 \\
\hline 12.51 & 1060. & 0.283 & 69033. & 4011. & 293.8 & -0.2 & 1.00 \\
\hline 12.51 & 1060. & 0.283 & 69084. & 3924. & 293.8 & -0.1 & 1.00 \\
\hline 12.51 & 1060 . & 0.283 & 69132. & 4020. & 293.8 & -0.1 & 1.00 \\
\hline 12.51 & 1060 . & 0.283 & 69177. & 3967. & 293.8 & -0.1 & 1.00 \\
\hline 12.51 & 1060. & 0.283 & 69225. & 3815. & 293.8 & -0.1 & 1.00 \\
\hline 12.51 & 1060. & 0.283 & 69274. & 3976. & 293.7 & $\begin{array}{l}-0.1 \\
\end{array}$ & 1.00 \\
\hline 12.51 & 1060. & 0.283 & 69322. & 3948. & 293.7 & 0.0 & 1.00 \\
\hline 13.38 & 1135. & 0.235 & 72093. & 3996. & 293.9 & -0.2 & 1.00 \\
\hline 13.38 & 1135. & 0.235 & 72139. & 4047. & 293.9 & -0.2 & 1.00 \\
\hline 13.38 & 1135. & 0.235 & 72186. & 4045. & 293.9 & \begin{tabular}{|c|}
-0.3 \\
\end{tabular} & 1.00 \\
\hline 13.38 & 1135. & 0.235 & 72236 . & 3938. & 293.9 & -0.3 & 1.00 \\
\hline 13.38 & 1135. & 0.235 & 72284. & 4061. & 294.0 & -0.3 & 1.00 \\
\hline 13.38 & 1135. & 0.235 & 72331. & 4062. & 293.9 & $\begin{array}{l}-0.3 \\
\end{array}$ & 1.00 \\
\hline 13.38 & 1135. & 0.235 & 72379 . & 3857. & 294.0 & $\begin{array}{l}-0.3 \\
\end{array}$ & 1.00 \\
\hline 13.38 & 1135. & 0.235 & 72428. & 4012. & 294.0 & $\begin{array}{l}-0.3 \\
\end{array}$ & 1.00 \\
\hline 13.38 & 1135. & 0.235 & 72482. & 3882. & 294.0 & $\begin{array}{l}-0.3 \\
\end{array}$ & 1.00 \\
\hline 13.38 & 1135. & 0.235 & 72531. & 4049. & 294.0 & -0.3 & 1.00 \\
\hline 13.38 & 1135. & 0.235 & 72577. & 3916. & 294.0 & $\begin{array}{l}-0.3 \\
\end{array}$ & 1.00 \\
\hline 13.38 & 1135. & 0.235 & 72621. & 4113. & 293.9 & $\begin{array}{l}-0.3 \\
\end{array}$ & 1.00 \\
\hline 13.38 & 1135. & 0.235 & 72668. & 3961. & 293.9 & -0.3 & 1.00 \\
\hline 13.78 & 1168. & 0.286 & 76231. & 4045. & 294.0 & $\begin{array}{l}-0.3 \\
\end{array}$ & 1.00 \\
\hline 13.78 & 1168. & 0.286 & 76278. & 3997. & 294.0 & $\begin{array}{l}-0.3 \\
\end{array}$ & 1.00 \\
\hline 13.78 & 1168. & 0.286 & 76326. & 3821. & 294.0 & -0.3 & 1.00 \\
\hline 13.78 & 1168. & 0.286 & 76375. & 3949. & 294.0 & $\begin{array}{l}-0.3 \\
\end{array}$ & 1.00 \\
\hline 13.78 & 1168. & 0.286 & 76421. & 3973. & 294.0 & $\begin{array}{l}-0.3 \\
\end{array}$ & 1.00 \\
\hline 13.78 & 1168. & 0.286 & 76475. & 4023. & 294.0 & $\begin{array}{l}-0.3 \\
\end{array}$ & 1.00 \\
\hline 13.78 & 1168. & 0.286 & 76523. & 4025. & 294.0 & $\begin{array}{l}-0.3 \\
\end{array}$ & 1.00 \\
\hline 13.78 & 1168. & 0.286 & 76568. & 3854. & 294.0 & $\begin{array}{l}-0.3 \\
\end{array}$ & 1.00 \\
\hline 13.78 & 1168. & 0.286 & 76613. & 3867. & 294.0 & $\begin{array}{l}-0.3 \\
\end{array}$ & 1.00 \\
\hline 13.78 & 1168. & 0.286 & 76659. & 4023. & 294.0 & -0.3 & 1.00 \\
\hline 13.78 & 1168. & 0.286 & 76707. & 4124. & 293.9 & -0.3 & 1.00 \\
\hline 13.78 & 1168. & 0.286 & 76755. & 4123. & 293.9 & $\begin{array}{l}-0.3 \\
\end{array}$ & 1.00 \\
\hline 13.78 & 1168. & 0.286 & 76826. & 4020. & 293.9 & -0.3 & 1.00 \\
\hline 14.05 & 1191. & 0.273 & 79482. & 4022. & 293.5 & 0.1 & 1.00 \\
\hline 14.05 & 1191. & 0.273 & 79525. & 3912. & 293.5 & 0.1 & 1.00 \\
\hline 14.05 & 1191. & 0.273 & 79573. & 3937. & 293.6 & 0.1 & 1.00 \\
\hline 14.05 & 1191. & 0.273 & 79620. & 3813. & 293.6 & 0.1 & 1.00 \\
\hline 14.05 & 1191. & 0.273 & 79661. & 3837. & 293.6 & 0.1 & 1.00 \\
\hline 14.05 & 1191. & 0.273 & 79706 . & 3803. & 293.6 & 0.0 & 1.00 \\
\hline 14.05 & 1191. & 0.273 & 79753. & 3842. & 293.7 & 0.0 & 1.00 \\
\hline 14.05 & 1191. & 0.273 & 79848. & 3988. & 293.7 & $\begin{array}{l}-0.1 \\
\end{array}$ & 1.00 \\
\hline 14.05 & 1191. & 0.273 & 79914. & 3956. & 293.8 & -0.1 & 1.00 \\
\hline 14.05 & 1191. & 0.273 & 79965. & 3970. & 293.8 & $\begin{array}{l}-0.1 \\
\end{array}$ & 1.00 \\
\hline 14.05 & 1191. & 0.273 & 80013. & 3924. & 293.8 & \begin{tabular}{l|l|}
-0.1 \\
\end{tabular} & 1.00 \\
\hline 14.05 & 1191. & 0.273 & 80063. & 3812. & 293.9 & -0.2 & 1.00 \\
\hline 14.05 & 1191. & 0.273 & 80110. & 3815. & 293.9 & $\begin{array}{l}-0.2 \\
\end{array}$ & 1.00 \\
\hline 15.02 & 1274. & 0.199 & 84094. & 3981. & 293.9 & -0.2 & 1.00 \\
\hline 15.02 & 1274. & 0.199 & 84157. & 4031. & 293.9 & -0.2 & 1.00 \\
\hline 15.02 & 1274. & 0.199 & 84212. & 3946. & 293.9 & $\begin{array}{l}-0.2 \\
\end{array}$ & 1.00 \\
\hline 15.02 & 1274. & 0.199 & 84261. & 4025. & 293.9 & -0.3 & 1.00 \\
\hline 15.02 & 1274. & 0.199 & 84310. & 3868. & 293.9 & $\begin{array}{l}-0.3 \\
\end{array}$ & 1.00 \\
\hline 15.02 & 1274. & 0.199 & 84356. & 3859. & 293.9 & $\begin{array}{l}-0.3 \\
\end{array}$ & 1.00 \\
\hline 15.02 & 1274. & 0.199 & 84404. & 3871. & 294.0 & -0.3 & 1.00 \\
\hline 15.02 & 1274. & 0.199 & 84450. & 4090. & 294.0 & $\begin{array}{l}-0.3 \\
\end{array}$ & 1.00 \\
\hline 15.02 & 1274. & 0.199 & 84493. & 3990. & 294.0 & $\begin{array}{l}-0.3 \\
\end{array}$ & 1.00 \\
\hline 15.02 & 1274. & 0.199 & 84538. & 4131. & 294.0 & -0.3 & 1.00 \\
\hline
\end{tabular}

\begin{tabular}{|c|c|c|c|c|c|c|c|}
\hline 15.02 & 1274. & 0.199 & 84588. & 4066. & 294.0 & -0.3 & 1.00 \\
\hline 15.02 & 1274. & 0.199 & 84634. & 3930. & 294.0 & -0.3 & 1.00 \\
\hline 15.02 & 1274. & 0.199 & 84678. & 4092. & 294.0 & -0.3 & 1.00 \\
\hline 23.80 & 2022. & 0.145 & 144804. & 4068. & 293.6 & 0.1 & 1.00 \\
\hline 23.80 & 2022. & 0.145 & 144848 . & 3958. & 293.6 & 0.1 & 1.00 \\
\hline 23.80 & 2022. & 0.145 & 144892 . & 3936. & 293.6 & 0.1 & 1.00 \\
\hline 23.80 & 2021. & 0.145 & 144934. & 3913. & 293.6 & 0.1 & 1.00 \\
\hline 23.80 & 2021. & 0.145 & 144980. & 3893. & 293.6 & 0.0 & 1.00 \\
\hline 23.80 & 2021. & 0.145 & 145026. & 4040 . & 293.7 & 0.0 & 1.00 \\
\hline 23.80 & 2021. & 0.145 & 145072. & 3873. & 293.7 & 0.0 & 1.00 \\
\hline 23.80 & 2021. & 0.145 & 145116. & 3899. & 293.7 & -0.1 & 1.00 \\
\hline 23.80 & 2021. & 0.145 & 145162. & 3984. & 293.7 & -0.1 & 1.00 \\
\hline 23.80 & 2021. & 0.145 & 145208. & 3915. & 293.8 & -0.1 & 1.00 \\
\hline 23.80 & 2021. & 0.145 & 145252. & 3804. & 293.8 & -0.1 & 1.00 \\
\hline 23.80 & 2021. & 0.145 & 145300 . & 4055. & 293.8 & -0.1 & 1.00 \\
\hline 23.80 & 2021. & 0.145 & 145348. & 3982. & 293.8 & -0.2 & 1.00 \\
\hline 24.33 & 2067. & 0.108 & 149421. & 3743. & 293.8 & -0.2 & 1.00 \\
\hline 24.33 & 2067. & 0.108 & 149465. & 3845. & 293.9 & -0.2 & 1.00 \\
\hline 24.33 & 2067. & 0.108 & 149507. & 3941. & 293.9 & -0.2 & 1.00 \\
\hline 24.33 & 2067. & 0.108 & 149552. & 3906. & 293.9 & -0.2 & 1.00 \\
\hline 24.33 & 2067. & 0.108 & 149600. & 4028. & 293.9 & -0.2 & 1.00 \\
\hline 24.33 & 2067. & 0.108 & 149642. & 4015. & 293.9 & -0.3 & 1.00 \\
\hline 24.33 & 2067. & 0.108 & 149690. & 4015. & 293.9 & $\begin{array}{l}-0.3 \\
\end{array}$ & 1.00 \\
\hline 24.33 & 2067. & 0.108 & 149739. & 3829. & 293.9 & $\begin{array}{l}-0.3 \\
\end{array}$ & 1.00 \\
\hline 24.33 & 2067. & 0.108 & 149785. & 4008. & 294.0 & -0.3 & 1.00 \\
\hline 24.33 & 2067. & 0.108 & 149833. & 4020. & 293.9 & -0.3 & 1.00 \\
\hline 24.33 & 2067. & 0.108 & 149877. & 4055. & 293.9 & $\begin{array}{c}-0.3 \\
\end{array}$ & 1.00 \\
\hline 24.33 & 2067. & 0.108 & 149922. & 3810. & 294.0 & -0.3 & 1.00 \\
\hline 24.33 & 2067. & 0.108 & 149971. & 3786. & 293.9 & -0.3 & 1.00 \\
\hline 32.28 & 2726. & 0.615 & 152899. & 3913. & 293.5 & 0.2 & 1.00 \\
\hline 32.28 & 2726. & 0.615 & 152946. & 3832. & 293.5 & 0.1 & 1.00 \\
\hline 32.28 & 2726. & 0.615 & 152989. & 3801. & 293.5 & 0.1 & 1.00 \\
\hline 32.28 & 2726. & 0.615 & 153034. & 3930. & 293.6 & 0.1 & 1.00 \\
\hline 32.28 & 2726. & 0.615 & 153079. & 3825. & 293.6 & 0.1 & 1.00 \\
\hline 32.28 & 2726. & 0.615 & 153127. & 3934. & 293.6 & 0.1 & 1.00 \\
\hline 32.28 & 2726. & 0.615 & 153172. & 3877. & 293.6 & 0.0 & 1.00 \\
\hline 32.28 & 2726. & 0.615 & 153215. & 3868. & 293.6 & 0.0 & 1.00 \\
\hline 32.28 & 2726. & 0.615 & 153260. & 3846. & 293.7 & 0.0 & 1.00 \\
\hline 32.28 & 2726. & 0.615 & 153300. & 3780. & 293.7 & 0.0 & 1.00 \\
\hline 32.28 & 2726. & 0.615 & 153351. & 3994. & 293.7 & -0.1 & 1.00 \\
\hline 32.28 & 2726. & 0.615 & 153398. & 3764. & 293.8 & -0.1 & 1.00 \\
\hline 32.28 & 2726. & 0.615 & 153445. & 3868. & 293.8 & -0.1 & 1.00 \\
\hline 25.27 & 2148. & 0.095 & 159952. & 3762. & 293.3 & 0.4 & 1.00 \\
\hline 25.27 & 2148. & 0.095 & 159997. & 3853. & 293.3 & 0.4 & 1.00 \\
\hline 25.27 & 2148. & 0.095 & 160042. & 3772. & 293.3 & 0.4 & 1.00 \\
\hline 25.27 & 2148. & 0.095 & 160086. & 3759. & 293.3 & 0.4 & 1.00 \\
\hline 25.27 & 2148. & 0.095 & 160128. & 3828. & 293.3 & 0.4 & 1.00 \\
\hline 25.27 & 2148. & 0.095 & 160170. & 3749. & 293.3 & 0.4 & 1.00 \\
\hline 25.27 & 2148. & 0.095 & 160221. & 3738. & 293.3 & 0.4 & 1.00 \\
\hline 25.27 & 2148. & 0.095 & 160264. & 3897. & 293.3 & 0.4 & 1.00 \\
\hline 25.27 & 2148. & 0.095 & 160307. & 3932. & 293.3 & 0.4 & 1.00 \\
\hline 25.27 & 2148. & 0.095 & 160352. & 3726. & 293.3 & 0.4 & 1.00 \\
\hline 25.27 & 2148. & 0.095 & 160399. & 3816. & 293.3 & 0.4 & 1.00 \\
\hline 25.27 & 2148. & 0.095 & 160443. & 3738. & 293.3 & 0.4 & 1.00 \\
\hline 25.27 & 2148. & 0.095 & 160489. & 3957. & 293.3 & 0.4 & 1.00 \\
\hline 25.68 & 2182. & 0.100 & 163842. & 3859. & 293.4 & 0.3 & 1.00 \\
\hline 25.68 & 2182. & 0.100 & 163886. & 3939. & 293.4 & 0.3 & 1.00 \\
\hline 25.68 & 2182. & 0.100 & 163935. & 3924. & 293.3 & 0.4 & 1.00 \\
\hline 25.68 & 2182. & 0.100 & 163984. & 3753. & 293.3 & 0.4 & 1.00 \\
\hline 25.68 & 2182. & 0.100 & 164030. & 3751. & 293.3 & 0.4 & 1.00 \\
\hline 25.68 & 2182. & 0.100 & 164077. & 3851. & 293.3 & 0.4 & 1.00 \\
\hline 25.68 & 2182. & 0.100 & 164121. & 3899. & 293.3 & 0.4 & 1.00 \\
\hline 25.68 & 2182. & 0.100 & 164166. & 3932. & 293.3 & 0.4 & 1.00 \\
\hline 25.68 & 2182. & 0.100 & 164214. & 3896. & 293.3 & 0.4 & 1.00 \\
\hline 25.68 & 2182. & 0.100 & 164262. & 3783. & 293.3 & 0.4 & 1.00 \\
\hline 25.68 & 2182. & 0.100 & 164307. & 3943. & 293.3 & 0.4 & 1.00 \\
\hline 25.68 & 2182. & 0.100 & 164351. & 3967. & 293.3 & 0.4 & 1.00 \\
\hline 25.68 & 2182. & 0.100 & 164398. & 3792. & 293.3 & 0.4 & 1.00 \\
\hline 24.46 & 2071. & 0.393 & 404186. & 3646. & 293.9 & -0.2 & 1.00 \\
\hline 24.46 & 2071. & 0.393 & 404233. & 3720. & 293.9 & -0.2 & 1.00 \\
\hline 24.46 & 2071. & 0.393 & 404282. & 3721. & 293.9 & -0.2 & 1.00 \\
\hline 24.46 & 2071. & 0.393 & 404327. & 3629. & 293.9 & -0.2 & 1.00 \\
\hline 24.46 & 2071. & 0.393 & 404373. & 3560. & 293.9 & -0.2 & 1.00 \\
\hline 24.46 & 2071. & 0.393 & 404418. & 3673. & 293.9 & -0.3 & 1.00 \\
\hline 24.46 & 2071. & 0.393 & 404463. & 3735. & 293.9 & -0.3 & 1.00 \\
\hline 24.46 & 2071. & 0.393 & 404509. & 3706. & 293.9 & -0.3 & 1.00 \\
\hline 24.46 & 2071. & 0.393 & 404554. & 3737. & 293.9 & -0.3 & 1.00 \\
\hline 24.46 & 2071. & 0.393 & 404599. & 3814. & 293.9 & -0.3 & 1.00 \\
\hline
\end{tabular}


Table H.2.10 Diesel contamination on oxidized copper surface for $\mathrm{Re}=5000$ and $x_{\mathrm{b}}=0.3 \%$ (file:trv45con2.tb2)

\begin{tabular}{|c|c|c|c|c|c|c|c|}
\hline $\begin{array}{c}l_{\mathrm{e}} \\
(\mu \mathrm{m})\end{array}$ & $\begin{array}{c}\Gamma \\
\left(\mathrm{kg} / \mathrm{m}^{2}\right) \\
\mathrm{X} 10^{5} \\
\end{array}$ & $\begin{array}{l}x_{\mathrm{b}} \\
(\%) \\
\end{array}$ & $\begin{array}{l}\text { Exposure } \\
\text { Time (s) }\end{array}$ & $\mathrm{Re}$ & 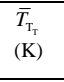 & $\begin{array}{c}T_{\mathrm{b}}-\bar{T}_{\mathrm{T}_{\mathrm{T}}} \\
(\mathrm{K})\end{array}$ & $\frac{F_{T_{\mathrm{b}}}}{F_{T_{\mathrm{T}}}}$ \\
\hline 0.38 & 32. & 0.287 & 4061. & 4853. & 292.4 & 1.3 & 1.00 \\
\hline 0.38 & 32. & 0.287 & 4103. & 4993. & 292.4 & 1.2 & 1.00 \\
\hline 0.38 & 32. & 0.287 & 4144. & 4998. & 292.5 & 1.2 & 1.00 \\
\hline 0.38 & 32. & 0.287 & 4187. & 5021. & 292.5 & 1.1 & 1.00 \\
\hline 0.38 & 32. & 0.287 & 4229. & 4870. & 292.6 & 1.1 & 1.00 \\
\hline 0.38 & 32. & 0.287 & 4271. & 4951. & 292.6 & 1.1 & 1.00 \\
\hline 0.38 & 32. & 0.287 & 4315. & 4955. & 292.6 & 1.0 & 1.00 \\
\hline 0.38 & 32. & 0.287 & 4360. & 5059. & 292.7 & 1.0 & 1.00 \\
\hline 0.38 & 32. & 0.287 & 4402. & 5106. & 292.7 & 1.0 & 1.00 \\
\hline 0.38 & 32. & 0.287 & 4444. & 4952. & 292.7 & 0.9 & 1.00 \\
\hline 0.38 & 32. & 0.287 & 4488. & 4859. & 292.8 & 0.9 & 1.00 \\
\hline 0.38 & 32. & 0.287 & 4530 . & 4736. & 292.8 & 0.8 & 1.00 \\
\hline 0.38 & 32. & 0.287 & 4574. & 5085. & 292.9 & 0.8 & 1.00 \\
\hline-0.06 & -5. & 0.346 & 4733. & 5079. & 293.0 & 0.7 & 1.00 \\
\hline-0.06 & -5. & 0.346 & 4776. & 5062. & 293.1 & 0.6 & 1.00 \\
\hline-0.06 & -5. & 0.346 & 4819. & 4892. & 293.1 & 0.6 & 1.00 \\
\hline-0.06 & -5. & 0.346 & 4862. & 5013. & 293.1 & 0.5 & 1.00 \\
\hline-0.06 & -5. & 0.346 & 4903. & 4882. & 293.2 & 0.5 & 1.00 \\
\hline-0.06 & -5. & 0.346 & 4948. & 5062. & 293.2 & 0.5 & 1.00 \\
\hline-0.06 & -5. & 0.346 & 4990. & 4912. & 293.3 & 0.4 & 1.00 \\
\hline-0.06 & -5. & 0.346 & 5032. & 5072. & 293.3 & 0.4 & 1.00 \\
\hline-0.06 & -5. & 0.346 & 5077. & 4922. & 293.4 & 0.3 & 1.00 \\
\hline-0.06 & -5. & 0.346 & 5121. & 5061. & 293.4 & 0.3 & 1.00 \\
\hline-0.06 & -5. & 0.346 & 5163. & 4930. & 293.4 & 0.2 & 1.00 \\
\hline-0.06 & -5. & 0.346 & 5205. & 4806. & 293.5 & 0.2 & 1.00 \\
\hline-0.06 & -5. & 0.346 & 5247. & 5074. & 293.5 & 0.1 & 1.00 \\
\hline 0.32 & 27. & 0.339 & 7217. & 5000. & 294.4 & -0.7 & 1.00 \\
\hline 0.32 & 27. & 0.339 & 7260. & 4980. & 294.4 & -0.7 & 1.00 \\
\hline 0.32 & 27. & 0.339 & 7312. & 5236. & 294.3 & -0.7 & 1.00 \\
\hline 0.32 & 27. & 0.339 & 7363. & 5034. & 294.3 & -0.7 & 1.00 \\
\hline 0.32 & 27. & 0.339 & 7418. & 5131. & 294.3 & -0.6 & 1.00 \\
\hline 0.32 & 27. & 0.339 & 7466. & 5232. & 294.3 & -0.6 & 1.00 \\
\hline 0.32 & 27. & 0.339 & 7510 . & 5186. & 294.3 & -0.6 & 1.00 \\
\hline 0.32 & 27. & 0.339 & 7553. & 5165. & 294.3 & -0.6 & 1.00 \\
\hline 0.32 & 27. & 0.339 & 7596. & 5225. & 294.3 & -0.6 & 1.00 \\
\hline 0.32 & 27. & 0.339 & 7638. & 5225. & 294.2 & -0.6 & 1.00 \\
\hline 0.32 & 27. & 0.339 & 7682. & 5265. & 294.2 & -0.6 & 1.00 \\
\hline 0.32 & 27. & 0.339 & 7724. & 5019. & 294.2 & -0.6 & 1.00 \\
\hline 0.32 & 27. & 0.339 & 7767. & 5077. & 294.2 & -0.5 & 1.00 \\
\hline 0.65 & 55. & 0.310 & 72772. & 5330. & 294.0 & -0.3 & 1.00 \\
\hline 0.65 & 55. & 0.310 & 72816. & 5331. & 294.1 & -0.4 & 1.00 \\
\hline 0.65 & 55. & 0.310 & 72863. & 5354. & 294.1 & -0.4 & 1.00 \\
\hline 0.65 & 55. & 0.310 & 72904. & 5161. & 294.1 & -0.4 & 1.00 \\
\hline 0.65 & 55. & 0.310 & 72945 . & 5466. & 294.1 & -0.4 & 1.00 \\
\hline 0.65 & 55. & 0.310 & 72988. & 5422. & 294.1 & -0.4 & 1.00 \\
\hline 0.65 & 55. & 0.310 & 73030. & 5314. & 294.1 & -0.4 & 1.00 \\
\hline 0.65 & 55. & 0.310 & 73071. & 5358. & 294.1 & -0.4 & 1.00 \\
\hline 0.65 & 55. & 0.310 & 73115. & 5314. & 294.1 & -0.4 & 1.00 \\
\hline 0.65 & 55. & 0.310 & 73157. & 5401. & 294.1 & -0.4 & 1.00 \\
\hline 0.65 & 55. & 0.310 & 73202. & 5446. & 294.1 & -0.4 & 1.00 \\
\hline 0.65 & 55. & 0.310 & 73245. & 5126. & 294.1 & -0.4 & 1.00 \\
\hline 0.65 & 55. & 0.310 & 73286 . & 5291. & 294.1 & -0.4 & 1.00 \\
\hline 0.48 & 41. & 0.313 & 73421. & 5166. & 294.1 & -0.4 & 1.00 \\
\hline 0.48 & 41. & 0.313 & 73462. & 5493. & 294.1 & -0.4 & 1.00 \\
\hline 0.48 & 41. & 0.313 & 73506. & 5443. & 294.1 & -0.4 & 1.00 \\
\hline 0.48 & 41. & 0.313 & 73548. & 5224. & 294.1 & -0.4 & 1.00 \\
\hline 0.48 & 41. & 0.313 & 73591. & 5182. & 294.1 & -0.4 & 1.00 \\
\hline 0.48 & 41. & 0.313 & 73636 . & 5203. & 294.0 & -0.4 & 1.00 \\
\hline 0.48 & 41. & 0.313 & 73680. & 5328. & 294.0 & -0.4 & 1.00 \\
\hline 0.48 & 41. & 0.313 & 73727. & 5373. & 294.0 & -0.3 & 1.00 \\
\hline 0.48 & 41. & 0.313 & 73771. & 5218. & 294.0 & -0.3 & 1.00 \\
\hline 0.48 & 41. & 0.313 & 73813. & 5348. & 294.0 & -0.3 & 1.00 \\
\hline 0.48 & 41. & 0.313 & 73855. & 5237. & 294.0 & -0.3 & 1.00 \\
\hline 0.48 & 41. & 0.313 & 73899 . & 5215. & 294.0 & -0.3 & 1.00 \\
\hline 0.48 & 41. & 0.313 & 73943. & 5523. & 294.0 & -0.3 & 1.00 \\
\hline 0.56 & 47. & 0.310 & 74123. & 5339. & 293.9 & -0.3 & 1.00 \\
\hline 0.56 & 47. & 0.310 & 74167. & 5165. & 293.9 & -0.2 & 1.00 \\
\hline 0.56 & 47. & 0.310 & 74210. & 5290. & 293.9 & -0.2 & 1.00 \\
\hline 0.56 & 47. & 0.310 & 74255. & 5264. & 293.9 & -0.2 & 1.00 \\
\hline 0.56 & 47. & 0.310 & 74298. & 5242. & 293.9 & -0.2 & 1.00 \\
\hline 0.56 & 47. & 0.310 & 74342. & 5136. & 293.8 & -0.2 & 1.00 \\
\hline 0.56 & 47. & 0.310 & 74388. & 5256. & 293.8 & -0.1 & 1.00 \\
\hline 0.56 & 47. & 0.310 & 74432. & 5299. & 293.8 & -0.1 & 1.00 \\
\hline 0.56 & 47. & 0.310 & 74477. & 5339. & 293.8 & -0.1 & 1.00 \\
\hline 0.56 & 47. & 0.310 & 74522. & 5009. & 293.8 & -0.1 & 1.00 \\
\hline 0.56 & 47. & 0.310 & 74567. & 5381. & 293.7 & -0.1 & 1.00 \\
\hline 0.56 & 47. & 0.310 & 74612. & 5224. & 293.7 & -0.1 & 1.00 \\
\hline
\end{tabular}

\begin{tabular}{|c|c|c|c|c|c|c|c|}
\hline 0.56 & 47. & 0.310 & 74658. & 5080. & 293.7 & 0.0 & 1.00 \\
\hline 1.34 & 113. & 0.234 & 78825. & 5174. & 293.9 & -0.3 & 1.00 \\
\hline 1.34 & 113. & 0.234 & 78868. & 5032. & 293.9 & -0.2 & 1.00 \\
\hline 1.34 & 113. & 0.234 & 78917. & 5407. & 293.9 & -0.2 & 1.00 \\
\hline 1.34 & 113. & 0.234 & 78963. & 5188. & 293.9 & -0.2 & 1.00 \\
\hline 1.34 & 113. & 0.234 & 79005. & 5493. & 293.9 & -0.2 & 1.00 \\
\hline 1.34 & 113. & 0.234 & 79047. & 5290. & 293.9 & -0.2 & 1.00 \\
\hline 1.34 & 113. & 0.234 & 79091. & 5221. & 293.8 & -0.2 & 1.00 \\
\hline 1.34 & 113. & 0.234 & 79137. & 5371. & 293.8 & -0.1 & 1.00 \\
\hline 1.34 & 113. & 0.234 & 79181. & 5219. & 293.8 & -0.1 & 1.00 \\
\hline 1.34 & 113. & 0.234 & 79224. & 4997. & 293.8 & -0.1 & 1.00 \\
\hline 1.34 & 113. & 0.234 & 79266. & 5387. & 293.7 & -0.1 & 1.00 \\
\hline 1.34 & 113. & 0.234 & 79306. & 5275. & 293.7 & -0.1 & 1.00 \\
\hline 1.34 & 113. & 0.234 & 79349. & 5008. & 293.7 & -0.1 & 1.00 \\
\hline 1.04 & 88. & 0.286 & 82735. & 5361. & 294.1 & -0.4 & 1.00 \\
\hline 1.04 & 88. & 0.286 & 82782. & 5274. & 294.1 & -0.4 & 1.00 \\
\hline 1.04 & 88. & 0.286 & 82827. & 5107. & 294.1 & -0.4 & 1.00 \\
\hline 1.04 & 88. & 0.286 & 82877. & 5230. & 294.1 & -0.4 & 1.00 \\
\hline 1.04 & 88. & 0.286 & 82921. & 5378. & 294.1 & -0.4 & 1.00 \\
\hline 1.04 & 88. & 0.286 & 82965. & 5424. & 294.1 & $\begin{array}{l}-0.4 \\
\end{array}$ & 1.00 \\
\hline 1.04 & 88. & 0.286 & 83007. & 5207. & 294.1 & -0.4 & 1.00 \\
\hline 1.04 & 88. & 0.286 & 83048. & 5122. & 294.1 & -0.4 & 1.00 \\
\hline 1.04 & 88. & 0.286 & 83093. & 5122. & 294.1 & -0.4 & 1.00 \\
\hline 1.04 & 88. & 0.286 & 83138. & 5267. & 294.0 & -0.4 & 1.00 \\
\hline 1.04 & 88. & 0.286 & 83182. & 5352. & 294.0 & -0.4 & 1.00 \\
\hline 1.04 & 88. & 0.286 & 83227. & 5287. & 294.0 & $\begin{array}{l}-0.3 \\
\end{array}$ & 1.00 \\
\hline 1.04 & 88. & 0.286 & 83271. & 5139. & 294.0 & $\begin{array}{c}-0.4 \\
\end{array}$ & 1.00 \\
\hline 1.18 & 100. & 0.297 & 85857. & 5372. & 293.5 & 0.2 & 1.00 \\
\hline 1.18 & 100. & 0.297 & 85901. & 5217. & 293.5 & 0.2 & 1.00 \\
\hline 1.18 & 100. & 0.297 & 85946. & 5098. & 293.5 & 0.2 & 1.00 \\
\hline 1.18 & 100. & 0.297 & 85989. & 5200. & 293.5 & 0.1 & 1.00 \\
\hline 1.18 & 100. & 0.297 & 86032. & 5289. & 293.5 & 0.1 & 1.00 \\
\hline 1.18 & 100. & 0.297 & 86077. & 5426. & 293.6 & 0.1 & 1.00 \\
\hline 1.18 & 100. & 0.297 & 86120. & 5338. & 293.6 & 0.1 & 1.00 \\
\hline 1.18 & 100. & 0.297 & 86164. & 5210. & 293.6 & 0.1 & 1.00 \\
\hline 1.18 & 100 . & 0.297 & 86211. & 5212. & 293.6 & 0.1 & 1.00 \\
\hline 1.18 & 100. & 0.297 & 86254. & 5412. & 293.6 & 0.0 & 1.00 \\
\hline 1.18 & 100. & 0.297 & 86299. & 5195. & 293.6 & 0.0 & 1.00 \\
\hline 1.18 & 100 . & 0.297 & 86344. & 5264. & 293.7 & 0.0 & 1.00 \\
\hline 1.18 & 100. & 0.297 & 86392. & 5399. & 293.7 & 0.0 & 1.00 \\
\hline 1.17 & 100. & 0.310 & 86538. & 5386. & 293.8 & -0.1 & 1.00 \\
\hline 1.17 & 100. & 0.310 & 86584. & 5278. & 293.8 & -0.1 & 1.00 \\
\hline 1.17 & 100. & 0.310 & 86626. & 5156. & 293.8 & -0.1 & 1.00 \\
\hline 1.17 & 100. & 0.310 & 86671. & 5351. & 293.8 & $\begin{array}{l}-0.2 \\
\end{array}$ & 1.00 \\
\hline 1.17 & 100. & 0.310 & 86721. & 5375. & 293.9 & -0.2 & 1.00 \\
\hline 1.17 & 100. & 0.310 & 86765. & 5471. & 293.9 & $\begin{array}{c}-0.3 \\
\end{array}$ & 1.00 \\
\hline 1.17 & 100. & 0.310 & 86810. & 5230. & 293.9 & -0.3 & 1.00 \\
\hline 1.17 & 100. & 0.310 & 86855. & 5316. & 293.9 & -0.3 & 1.00 \\
\hline 1.17 & 100. & 0.310 & 86900. & 5387. & 294.0 & -0.3 & 1.00 \\
\hline 1.17 & 100. & 0.310 & 86947. & 5154. & 294.0 & -0.3 & 1.00 \\
\hline 1.17 & 100. & 0.310 & 86992. & 5258. & 294.0 & $\begin{array}{l}-0.3 \\
\end{array}$ & 1.00 \\
\hline 1.17 & 100. & 0.310 & 87035 . & 5373. & 294.0 & $\begin{array}{l}-0.3 \\
\end{array}$ & 1.00 \\
\hline 1.17 & 100. & 0.310 & 87081. & 5439. & 294.0 & -0.4 & 1.00 \\
\hline 1.44 & 122. & 0.301 & 90303. & 5249. & 293.4 & 0.3 & 1.00 \\
\hline 1.44 & 122. & 0.301 & 90351. & 5360 . & 293.4 & 0.3 & 1.00 \\
\hline 1.44 & 122. & 0.301 & 90403. & 5255. & 293.4 & 0.3 & 1.00 \\
\hline 1.44 & 122. & 0.301 & 90451. & 5387. & 293.4 & 0.2 & 1.00 \\
\hline 1.44 & 122. & 0.301 & 90497. & 5232. & 293.4 & 0.2 & 1.00 \\
\hline 1.44 & 122. & 0.301 & 90539. & 5298. & 293.4 & 0.2 & 1.00 \\
\hline 1.44 & 122. & 0.301 & 90584. & 5091. & 293.4 & 0.2 & 1.00 \\
\hline 1.44 & 122. & 0.301 & 90629. & 5215. & 293.5 & 0.2 & 1.00 \\
\hline 1.44 & 122. & 0.301 & 90673. & 5262. & 293.5 & 0.2 & 1.00 \\
\hline 1.44 & 122. & 0.301 & 90715. & 4999. & 293.5 & 0.2 & 1.00 \\
\hline 1.44 & 122. & 0.301 & 90758. & 5307. & 293.5 & 0.1 & 1.00 \\
\hline 1.44 & 122. & 0.301 & 90803. & 5080. & 293.5 & 0.1 & 1.00 \\
\hline 1.44 & 122. & 0.301 & 90848. & 5270. & 293.5 & 0.1 & 1.00 \\
\hline 1.67 & 142. & 0.304 & 93827. & 5326. & 293.6 & 0.0 & 1.00 \\
\hline 1.67 & 142. & 0.304 & 93869. & 5258. & 293.6 & 0.0 & 1.00 \\
\hline 1.67 & 142. & 0.304 & 93921. & 5256. & 293.6 & 0.1 & 1.00 \\
\hline 1.67 & 142. & 0.304 & 93972. & 5027. & 293.6 & 0.1 & 1.00 \\
\hline 1.67 & 142. & 0.304 & 94019. & 4966. & 293.5 & 0.1 & 1.00 \\
\hline 1.67 & 142. & 0.304 & 94097. & 5330. & 293.5 & 0.2 & 1.00 \\
\hline 1.67 & 142. & 0.304 & 94223. & 5214. & 293.4 & 0.2 & 1.00 \\
\hline 1.67 & 142. & 0.304 & 94280. & 5008. & 293.4 & 0.3 & 1.00 \\
\hline 1.67 & 142. & 0.304 & 94342. & 5128. & 293.4 & 0.3 & 1.00 \\
\hline 1.67 & 142. & 0.304 & 94392. & 5023. & 293.4 & 0.3 & 1.00 \\
\hline 1.67 & 142. & 0.304 & 94438. & 5142. & 293.4 & 0.3 & 1.00 \\
\hline 1.67 & 142. & 0.304 & 94482. & 5249. & 293.4 & 0.3 & 1.00 \\
\hline 1.67 & 142. & 0.304 & 94536. & 5039. & 293.4 & 0.3 & 1.00 \\
\hline 1.81 & 153. & 0.299 & 94678. & 5099. & 293.4 & 0.3 & 1.00 \\
\hline
\end{tabular}


Table H.2.11 Diesel contamination on oxidized copper surface for $\mathrm{Re}=7000$ and $x_{\mathrm{b}}=0.3 \%$ (file:trv6con2.tb2)

\begin{tabular}{|c|c|c|c|c|c|c|c|}
\hline $\begin{array}{c}l_{\mathrm{e}} \\
(\mu \mathrm{m})\end{array}$ & $\begin{array}{c}\Gamma \\
\left(\mathrm{kg} / \mathrm{m}^{2}\right) \\
\mathrm{X} 10^{5}\end{array}$ & $\begin{array}{l}x_{\mathrm{b}} \\
(\%)\end{array}$ & $\begin{array}{l}\text { Exposure } \\
\text { Time (s) }\end{array}$ & $\mathrm{Re}$ & $\begin{array}{l}\bar{T}_{\mathrm{T}_{\mathrm{T}}} \\
(\mathrm{K})\end{array}$ & $\begin{array}{c}T_{\mathrm{b}}-\bar{T}_{\mathrm{T}_{\mathrm{T}}} \\
(\mathrm{K})\end{array}$ & $\frac{F_{T_{b}}}{F_{T_{\mathrm{T}}}}$ \\
\hline 0.38 & 32. & 0.287 & 4061. & 4853. & 292.4 & 1.3 & 1.00 \\
\hline 0.38 & 32. & 0.287 & 4103. & 4993. & 292.4 & 1.2 & 1.00 \\
\hline 0.38 & 32. & 0.287 & 4144. & 4998. & 292.5 & 1.2 & 1.00 \\
\hline 0.38 & 32. & 0.287 & 4187. & 5021. & 292.5 & 1.1 & 1.00 \\
\hline 0.38 & 32. & 0.287 & 4229. & 4870. & 292.6 & 1.1 & 1.00 \\
\hline 0.38 & 32. & 0.287 & 4271. & 4951. & 292.6 & 1.1 & 1.00 \\
\hline 0.38 & 32. & 0.287 & 4315. & 4955. & 292.6 & 1.0 & 1.00 \\
\hline 0.38 & 32. & 0.287 & 4360 . & 5059. & 292.7 & 1.0 & 1.00 \\
\hline 0.38 & 32. & 0.287 & 4402. & 5106. & 292.7 & 1.0 & 1.00 \\
\hline 0.38 & 32. & 0.287 & 4444. & 4952. & 292.7 & 0.9 & 1.00 \\
\hline 0.38 & 32. & 0.287 & 4488. & 4859. & 292.8 & 0.9 & 1.00 \\
\hline 0.38 & 32. & 0.287 & 4530. & 4736. & 292.8 & 0.8 & 1.00 \\
\hline 0.38 & 32. & 0.287 & 4574. & 5085. & 292.9 & 0.8 & 1.00 \\
\hline-0.06 & -5. & 0.346 & 4733. & 5079. & 293.0 & 0.7 & 1.00 \\
\hline-0.06 & -5. & 0.346 & 4776. & 5062. & 293.1 & 0.6 & 1.00 \\
\hline-0.06 & -5. & 0.346 & 4819. & 4892. & 293.1 & 0.6 & 1.00 \\
\hline-0.06 & -5. & 0.346 & 4862. & 5013. & 293.1 & 0.5 & 1.00 \\
\hline-0.06 & -5. & 0.346 & 4903. & 4882. & 293.2 & 0.5 & 1.00 \\
\hline-0.06 & -5. & 0.346 & 4948. & 5062. & 293.2 & 0.5 & 1.00 \\
\hline-0.06 & -5. & 0.346 & 4990. & 4912. & 293.3 & 0.4 & 1.00 \\
\hline-0.06 & -5. & 0.346 & 5032. & 5072 . & 293.3 & 0.4 & 1.00 \\
\hline-0.06 & -5. & 0.346 & 5077. & 4922. & 293.4 & 0.3 & 1.00 \\
\hline-0.06 & -5. & 0.346 & 5121. & 5061. & 293.4 & 0.3 & 1.00 \\
\hline-0.06 & -5. & 0.346 & 5163. & 4930. & 293.4 & 0.2 & 1.00 \\
\hline-0.06 & -5. & 0.346 & 5205. & 4806. & 293.5 & 0.2 & 1.00 \\
\hline-0.06 & -5. & 0.346 & 5247. & 5074. & 293.5 & 0.1 & 1.00 \\
\hline 0.32 & 27. & 0.339 & 7217. & 5000 . & 294.4 & -0.7 & 1.00 \\
\hline 0.32 & 27. & 0.339 & 7260. & 4980. & 294.4 & -0.7 & 1.00 \\
\hline 0.32 & 27. & 0.339 & 7312. & 5236. & 294.3 & -0.7 & 1.00 \\
\hline 0.32 & 27. & 0.339 & 7363. & 5034. & 294.3 & -0.7 & 1.00 \\
\hline 0.32 & 27. & 0.339 & 7418. & 5131. & 294.3 & -0.6 & 1.00 \\
\hline 0.32 & 27. & 0.339 & 7466. & 5232. & 294.3 & -0.6 & 1.00 \\
\hline 0.32 & 27. & 0.339 & 7510. & 5186. & 294.3 & -0.6 & 1.00 \\
\hline 0.32 & 27. & 0.339 & 7553. & 5165. & 294.3 & -0.6 & 1.00 \\
\hline 0.32 & 27. & 0.339 & 7596. & 5225. & 294.3 & -0.6 & 1.00 \\
\hline 0.32 & 27. & 0.339 & 7638. & 5225. & 294.2 & -0.6 & 1.00 \\
\hline 0.32 & 27. & 0.339 & 7682. & 5265. & 294.2 & -0.6 & 1.00 \\
\hline 0.32 & 27. & 0.339 & 7724. & 5019. & 294.2 & -0.6 & 1.00 \\
\hline 0.32 & 27. & 0.339 & 7767. & 5077. & 294.2 & -0.5 & 1.00 \\
\hline 0.65 & 55. & 0.310 & 72772 . & 5330. & 294.0 & -0.3 & 1.00 \\
\hline 0.65 & 55. & 0.310 & 72816. & 5331. & 294.1 & -0.4 & 1.00 \\
\hline 0.65 & 55. & 0.310 & 72863. & 5354. & 294.1 & -0.4 & 1.00 \\
\hline 0.65 & 55. & 0.310 & 72904. & 5161. & 294.1 & -0.4 & 1.00 \\
\hline 0.65 & 55. & 0.310 & 72945. & 5466. & 294.1 & -0.4 & 1.00 \\
\hline 0.65 & 55. & 0.310 & 72988 . & 5422. & 294.1 & -0.4 & 1.00 \\
\hline 0.65 & 55. & 0.310 & 73030 . & 5314. & 294.1 & -0.4 & 1.00 \\
\hline 0.65 & 55. & 0.310 & 73071. & 5358. & 294.1 & -0.4 & 1.00 \\
\hline 0.65 & 55. & 0.310 & 73115. & 5314. & 294.1 & -0.4 & 1.00 \\
\hline 0.65 & 55. & 0.310 & 73157. & 5401. & 294.1 & -0.4 & 1.00 \\
\hline 0.65 & 55. & 0.310 & 73202. & 5446. & 294.1 & -0.4 & 1.00 \\
\hline 0.65 & 55. & 0.310 & 73245 . & 5126. & 294.1 & -0.4 & 1.00 \\
\hline 0.65 & 55. & 0.310 & 73286. & 5291. & 294.1 & -0.4 & 1.00 \\
\hline 0.48 & 41. & 0.313 & 73421. & 5166. & 294.1 & -0.4 & 1.00 \\
\hline 0.48 & 41. & 0.313 & 73462. & 5493. & 294.1 & -0.4 & 1.00 \\
\hline 0.48 & 41. & 0.313 & 73506 . & 5443. & 294.1 & -0.4 & 1.00 \\
\hline 0.48 & 41. & 0.313 & 73548. & 5224. & 294.1 & -0.4 & 1.00 \\
\hline 0.48 & 41. & 0.313 & 73591. & 5182. & 294.1 & -0.4 & 1.00 \\
\hline 0.48 & 41. & 0.313 & 73636. & 5203. & 294.0 & -0.4 & 1.00 \\
\hline 0.48 & 41. & 0.313 & 73680 . & 5328. & 294.0 & -0.4 & 1.00 \\
\hline 0.48 & 41. & 0.313 & 73727. & 5373. & 294.0 & -0.3 & 1.00 \\
\hline 0.48 & 41. & 0.313 & 73771. & 5218. & 294.0 & -0.3 & 1.00 \\
\hline 0.48 & 41. & 0.313 & 73813. & 5348. & 294.0 & -0.3 & 1.00 \\
\hline 0.48 & 41. & 0.313 & 73855. & 5237. & 294.0 & -0.3 & 1.00 \\
\hline 0.48 & 41. & 0.313 & 73899. & 5215. & 294.0 & -0.3 & 1.00 \\
\hline 0.48 & 41. & 0.313 & 73943. & 5523. & 294.0 & -0.3 & 1.00 \\
\hline 0.56 & 47. & 0.310 & 74123. & 5339. & 293.9 & -0.3 & 1.00 \\
\hline 0.56 & 47. & 0.310 & 74167. & 5165. & 293.9 & -0.2 & 1.00 \\
\hline 0.56 & 47. & 0.310 & 74210 . & 5290. & 293.9 & -0.2 & 1.00 \\
\hline 0.56 & 47. & 0.310 & 74255 . & 5264. & 293.9 & -0.2 & 1.00 \\
\hline 0.56 & 47. & 0.310 & 74298. & 5242. & 293.9 & -0.2 & 1.00 \\
\hline 0.56 & 47. & 0.310 & 74342. & 5136. & 293.8 & -0.2 & 1.00 \\
\hline 0.56 & 47. & 0.310 & 74388. & 5256. & 293.8 & -0.1 & 1.00 \\
\hline 0.56 & 47. & 0.310 & 74432. & 5299. & 293.8 & -0.1 & 1.00 \\
\hline 0.56 & 47. & 0.310 & 74477. & 5339. & 293.8 & -0.1 & 1.00 \\
\hline
\end{tabular}

\begin{tabular}{|c|c|c|c|c|c|c|c|}
\hline 0.56 & 47. & 0.310 & 74522. & 5009. & 293.8 & -0.1 & 1.00 \\
\hline 0.56 & 47. & 0.310 & 74567. & 5381. & 293.7 & -0.1 & 1.00 \\
\hline 0.56 & 47. & 0.310 & 74612. & 5224. & 293.7 & -0.1 & 1.00 \\
\hline 0.56 & 47. & 0.310 & 74658. & 5080. & 293.7 & 0.0 & 1.00 \\
\hline 1.34 & 113. & 0.234 & 78825 . & 5174. & 293.9 & -0.3 & 1.00 \\
\hline 1.34 & 113. & 0.234 & 78868. & 5032. & 293.9 & -0.2 & 1.00 \\
\hline 1.34 & 113. & 0.234 & 78917. & 5407. & 293.9 & -0.2 & 1.00 \\
\hline 1.34 & 113. & 0.234 & 78963. & 5188. & 293.9 & -0.2 & 1.00 \\
\hline 1.34 & 113. & 0.234 & 79005. & 5493. & 293.9 & -0.2 & 1.00 \\
\hline 1.34 & 113. & 0.234 & 79047. & 5290. & 293.9 & -0.2 & 1.00 \\
\hline 1.34 & 113. & 0.234 & 79091. & 5221. & 293.8 & -0.2 & 1.00 \\
\hline 1.34 & 113. & 0.234 & 79137. & 5371. & 293.8 & -0.1 & 1.00 \\
\hline 1.34 & 113. & 0.234 & 79181. & 5219. & 293.8 & -0.1 & 1.00 \\
\hline 1.34 & 113. & 0.234 & 79224. & 4997. & 293.8 & -0.1 & 1.00 \\
\hline 1.34 & 113. & 0.234 & 79266. & 5387. & 293.7 & -0.1 & 1.00 \\
\hline 1.34 & 113. & 0.234 & 79306. & 5275. & 293.7 & -0.1 & 1.00 \\
\hline 1.34 & 113. & 0.234 & 79349. & 5008. & 293.7 & -0.1 & 1.00 \\
\hline 1.04 & 88. & 0.286 & 82735. & 5361. & 294.1 & -0.4 & 1.00 \\
\hline 1.04 & 88. & 0.286 & 82782. & 5274. & 294.1 & -0.4 & 1.00 \\
\hline 1.04 & 88. & 0.286 & 82827. & 5107. & 294.1 & -0.4 & 1.00 \\
\hline 1.04 & 88. & 0.286 & 82877. & 5230. & 294.1 & -0.4 & 1.00 \\
\hline 1.04 & 88. & 0.286 & 82921. & 5378. & 294.1 & -0.4 & 1.00 \\
\hline 1.04 & 88. & 0.286 & 82965. & 5424. & 294.1 & -0.4 & 1.00 \\
\hline 1.04 & 88. & 0.286 & 83007. & 5207. & 294.1 & -0.4 & 1.00 \\
\hline 1.04 & 88. & 0.286 & 83048. & 5122. & 294.1 & -0.4 & 1.00 \\
\hline 1.04 & 88. & 0.286 & 83093. & 5122. & 294.1 & -0.4 & 1.00 \\
\hline 1.04 & 88. & 0.286 & 83138. & 5267. & 294.0 & -0.4 & 1.00 \\
\hline 1.04 & 88. & 0.286 & 83182. & 5352. & 294.0 & -0.4 & 1.00 \\
\hline 1.04 & 88. & 0.286 & 83227. & 5287. & 294.0 & -0.3 & 1.00 \\
\hline 1.04 & 88. & 0.286 & 83271. & 5139. & 294.0 & -0.4 & 1.00 \\
\hline 1.18 & 100. & 0.297 & 85857. & 5372. & 293.5 & 0.2 & 1.00 \\
\hline 1.18 & 100. & 0.297 & 85901. & 5217. & 293.5 & 0.2 & 1.00 \\
\hline 1.18 & 100. & 0.297 & 85946. & 5098. & 293.5 & 0.2 & 1.00 \\
\hline 1.18 & 100. & 0.297 & 85989. & 5200. & 293.5 & 0.1 & 1.00 \\
\hline 1.18 & 100. & 0.297 & 86032. & 5289. & 293.5 & 0.1 & 1.00 \\
\hline 1.18 & 100. & 0.297 & 86077. & 5426. & 293.6 & 0.1 & 1.00 \\
\hline 1.18 & 100. & 0.297 & 86120. & 5338. & 293.6 & 0.1 & 1.00 \\
\hline 1.18 & 100. & 0.297 & 86164. & 5210. & 293.6 & 0.1 & 1.00 \\
\hline 1.18 & 100. & 0.297 & 86211. & 5212. & 293.6 & 0.1 & 1.00 \\
\hline 1.18 & 100. & 0.297 & 86254. & 5412. & 293.6 & 0.0 & 1.00 \\
\hline 1.18 & 100. & 0.297 & 86299. & 5195. & 293.6 & 0.0 & 1.00 \\
\hline 1.18 & 100. & 0.297 & 86344. & 5264. & 293.7 & 0.0 & 1.00 \\
\hline 1.18 & 100. & 0.297 & 86392. & 5399. & 293.7 & 0.0 & 1.00 \\
\hline 1.17 & 100. & 0.310 & 86538. & 5386. & 293.8 & -0.1 & 1.00 \\
\hline 1.17 & 100. & 0.310 & 86584. & 5278. & 293.8 & -0.1 & 1.00 \\
\hline 1.17 & 100. & 0.310 & 86626. & 5156. & 293.8 & -0.1 & 1.00 \\
\hline 1.17 & 100. & 0.310 & 86671. & 5351. & 293.8 & -0.2 & 1.00 \\
\hline 1.17 & 100. & 0.310 & 86721. & 5375. & 293.9 & -0.2 & 1.00 \\
\hline 1.17 & 100. & 0.310 & 86765. & 5471. & 293.9 & -0.3 & 1.00 \\
\hline 1.17 & 100. & 0.310 & 86810. & 5230. & 293.9 & -0.3 & 1.00 \\
\hline 1.17 & 100. & 0.310 & 86855. & 5316. & 293.9 & -0.3 & 1.00 \\
\hline 1.17 & 100. & 0.310 & 86900. & 5387. & 294.0 & -0.3 & 1.00 \\
\hline 1.17 & 100. & 0.310 & 86947. & 5154. & 294.0 & -0.3 & 1.00 \\
\hline 1.17 & 100. & 0.310 & 86992. & 5258. & 294.0 & -0.3 & 1.00 \\
\hline 1.17 & 100. & 0.310 & 87035. & 5373. & 294.0 & -0.3 & 1.00 \\
\hline 1.17 & 100. & 0.310 & 87081. & 5439. & 294.0 & -0.4 & 1.00 \\
\hline 1.44 & 122. & 0.301 & 90303. & 5249. & 293.4 & 0.3 & 1.00 \\
\hline 1.44 & 122. & 0.301 & 90351. & 5360. & 293.4 & 0.3 & 1.00 \\
\hline 1.44 & 122. & 0.301 & 90403. & 5255. & 293.4 & 0.3 & 1.00 \\
\hline 1.44 & 122. & 0.301 & 90451. & 5387. & 293.4 & 0.2 & 1.00 \\
\hline 1.44 & 122. & 0.301 & 90497. & 5232. & 293.4 & 0.2 & 1.00 \\
\hline 1.44 & 122. & 0.301 & 90539. & 5298. & 293.4 & 0.2 & 1.00 \\
\hline 1.44 & 122. & 0.301 & 90584. & 5091. & 293.4 & 0.2 & 1.00 \\
\hline 1.44 & 122. & 0.301 & 90629. & 5215. & 293.5 & 0.2 & 1.00 \\
\hline 1.44 & 122. & 0.301 & 90673. & 5262. & 293.5 & 0.2 & 1.00 \\
\hline 1.44 & 122. & 0.301 & 90715. & 4999. & 293.5 & 0.2 & 1.00 \\
\hline 1.44 & 122. & 0.301 & 90758. & 5307. & 293.5 & 0.1 & 1.00 \\
\hline 1.44 & 122. & 0.301 & 90803. & 5080. & 293.5 & 0.1 & 1.00 \\
\hline 1.44 & 122. & 0.301 & 90848. & 5270. & 293.5 & 0.1 & 1.00 \\
\hline 1.67 & 142. & 0.304 & 93827. & 5326. & 293.6 & 0.0 & 1.00 \\
\hline 1.67 & 142. & 0.304 & 93869. & 5258. & 293.6 & 0.0 & 1.00 \\
\hline 1.67 & 142. & 0.304 & 93921. & 5256. & 293.6 & 0.1 & 1.00 \\
\hline 1.67 & 142. & 0.304 & 93972. & 5027. & 293.6 & 0.1 & 1.00 \\
\hline 1.67 & 142. & 0.304 & 94019. & 4966. & 293.5 & 0.1 & 1.00 \\
\hline 1.67 & 142. & 0.304 & 94097. & 5330. & 293.5 & 0.2 & 1.00 \\
\hline 1.67 & 142. & 0.304 & 94223. & 5214. & 293.4 & 0.2 & 1.00 \\
\hline 1.67 & 142. & 0.304 & 94280. & 5008. & 293.4 & 0.3 & 1.00 \\
\hline
\end{tabular}


Table H.2.12 Tap water flushing after $\mathrm{Re}=5000$ contamination tests at $x_{\mathrm{b}}=0.3 \%$ (file:flsh45c2.tb2)

\begin{tabular}{|c|c|c|c|c|c|c|}
\hline $\begin{array}{c}l_{\mathrm{e}} \\
(\mu \mathrm{m})\end{array}$ & $\begin{array}{c}\Gamma \\
\left(\mathrm{kg} / \mathrm{m}^{2}\right) \\
\mathrm{X} 10^{5}\end{array}$ & $\begin{array}{l}x_{\mathrm{b}} \\
(\%)\end{array}$ & $\begin{array}{c}\text { Exposure } \\
\text { Time (s) }\end{array}$ & $\begin{array}{l}\bar{T}_{\mathrm{T}_{\mathrm{T}}} \\
(\mathrm{K})\end{array}$ & $\begin{array}{c}T_{\mathrm{b}}-\bar{T}_{\mathrm{T}_{\mathrm{T}}} \\
(\mathrm{K})\end{array}$ & $\frac{F_{T_{\mathrm{b}}}}{F_{T_{\mathrm{T}}}}$ \\
\hline 0.15 & 13. & -0.007 & 402. & 293.2 & 0.5 & 1.00 \\
\hline 0.15 & 13. & -0.007 & 453. & 292.6 & 1.1 & 1.00 \\
\hline 0.15 & 13. & -0.007 & 506. & 292.0 & 1.7 & 1.00 \\
\hline 0.15 & 13. & -0.007 & 557. & 291.5 & 2.2 & 1.00 \\
\hline 0.15 & 13. & -0.007 & 610. & 291.0 & 2.7 & 1.00 \\
\hline 0.15 & 13. & -0.007 & 663. & 290.6 & 3.1 & 1.00 \\
\hline 0.15 & 13. & -0.007 & 719. & 290.1 & 3.6 & 1.01 \\
\hline 0.15 & 13. & -0.007 & 775. & 289.6 & 4.0 & 1.01 \\
\hline 0.15 & 13. & -0.007 & 832. & 289.2 & 4.5 & 1.01 \\
\hline 0.15 & 13. & -0.007 & 886. & 288.9 & 4.8 & 1.01 \\
\hline 0.15 & 13. & -0.007 & 938. & 288.7 & 5.0 & 1.01 \\
\hline 0.15 & 13. & -0.007 & 995. & 288.5 & 5.2 & 1.01 \\
\hline 0.15 & 13. & -0.007 & 1053. & 288.3 & 5.4 & 1.01 \\
\hline 0.03 & 3. & 0.007 & 3736. & 286.4 & 7.3 & 1.01 \\
\hline 0.03 & 3. & 0.007 & 3787. & 286.4 & 7.3 & 1.01 \\
\hline 0.03 & 3. & 0.007 & 3842. & 286.4 & 7.3 & 1.01 \\
\hline 0.03 & 3. & 0.007 & 3902. & 286.4 & 7.3 & 1.01 \\
\hline 0.03 & 3. & 0.007 & 3958. & 286.4 & 7.3 & 1.01 \\
\hline 0.03 & 3. & 0.007 & 4013. & 286.4 & 7.3 & 1.01 \\
\hline 0.03 & 3. & 0.007 & 4070 . & 286.4 & 7.3 & 1.01 \\
\hline 0.03 & 3. & 0.007 & 4124. & 286.4 & 7.3 & 1.01 \\
\hline 0.03 & 3. & 0.007 & 4180. & 286.4 & 7.3 & 1.01 \\
\hline 0.03 & 3. & 0.007 & 4234. & 286.4 & 7.3 & 1.01 \\
\hline 0.03 & 3. & 0.007 & 4294. & 286.4 & 7.3 & 1.01 \\
\hline 0.03 & 3. & 0.007 & 4349. & 286.4 & 7.3 & 1.01 \\
\hline 0.03 & 3. & 0.007 & 4406. & 286.4 & 7.3 & 1.01 \\
\hline-0.40 & -34. & -0.004 & 64014. & 287.3 & 6.4 & 1.01 \\
\hline-0.40 & -34. & -0.004 & 64064. & 287.3 & 6.3 & 1.01 \\
\hline-0.40 & -34. & -0.004 & 64118. & 287.3 & 6.3 & 1.01 \\
\hline-0.40 & -34. & -0.004 & 64176. & 287.3 & 6.4 & 1.01 \\
\hline-0.40 & -34. & -0.004 & 64239. & 287.3 & 6.4 & 1.01 \\
\hline-0.40 & -34. & -0.004 & 64292. & 287.3 & 6.3 & 1.01 \\
\hline-0.40 & -34. & -0.004 & 64346. & 287.3 & 6.4 & 1.01 \\
\hline-0.40 & -34. & -0.004 & 64398. & 287.3 & 6.3 & 1.01 \\
\hline-0.40 & -34. & -0.004 & 64452. & 287.3 & 6.4 & 1.01 \\
\hline-0.40 & -34 & -0.004 & 64508. & 287.3 & 6.3 & 1.01 \\
\hline-0.40 & -34. & -0.004 & 64560. & 287.3 & 6.3 & 1.01 \\
\hline-0.40 & -34. & -0.004 & 64612. & 287.3 & 6.3 & 1.01 \\
\hline-0.40 & -34. & -0.004 & 64667. & 287.3 & 6.3 & 1.01 \\
\hline-0.19 & -16. & -0.003 & 64866. & 287.3 & 6.3 & 1.01 \\
\hline-0.19 & -16. & -0.003 & 64920. & 287.3 & 6.3 & 1.01 \\
\hline-0.19 & -16. & -0.003 & 64975. & 287.3 & 6.3 & 1.01 \\
\hline-0.19 & -16. & -0.003 & 65027. & 287.3 & 6.3 & 1.01 \\
\hline-0.19 & -16. & -0.003 & 65082. & 287.3 & 6.3 & 1.01 \\
\hline-0.19 & -16. & -0.003 & 65135. & 287.3 & 6.3 & 1.01 \\
\hline-0.19 & -16. & -0.003 & 65188. & 287.3 & 6.3 & 1.01 \\
\hline-0.19 & -16. & -0.003 & 65245. & 287.3 & 6.3 & 1.01 \\
\hline-0.19 & -16. & -0.003 & 65300. & 287.3 & 6.3 & 1.01 \\
\hline-0.19 & -16. & -0.003 & 65351. & 287.3 & 6.3 & 1.01 \\
\hline-0.19 & -16. & -0.003 & 65405. & 287.3 & 6.3 & 1.01 \\
\hline-0.19 & -16. & -0.003 & 65458. & 287.3 & 6.3 & 1.01 \\
\hline-0.19 & -16. & -0.003 & 65513. & 287.3 & 6.3 & 1.01 \\
\hline-0.39 & -34. & -0.004 & 67977. & 287.5 & 6.2 & 1.01 \\
\hline-0.39 & -34. & -0.004 & 68028. & 287.5 & 6.2 & 1.01 \\
\hline-0.39 & -34. & -0.004 & 68086. & 287.5 & 6.2 & 1.01 \\
\hline-0.39 & -34. & -0.004 & 68139. & 287.5 & 6.2 & 1.01 \\
\hline-0.39 & -34. & -0.004 & 68190. & 287.5 & 6.2 & 1.01 \\
\hline-0.39 & -34. & -0.004 & 68242. & 287.5 & 6.2 & 1.01 \\
\hline-0.39 & -34. & -0.004 & 68297. & 287.5 & 6.2 & 1.01 \\
\hline-0.39 & -34. & -0.004 & 68349 . & 287.5 & 6.2 & 1.01 \\
\hline-0.39 & -34. & -0.004 & 68402 . & 287.5 & 6.2 & 1.01 \\
\hline-0.39 & -34 & -0.004 & 68453. & 287.5 & 6.2 & 1.01 \\
\hline-0.39 & -34. & -0.004 & 68504. & 287.5 & 6.2 & 1.01 \\
\hline-0.39 & -34. & -0.004 & 68556. & 287.5 & 6.2 & 1.01 \\
\hline-0.39 & -34. & -0.004 & 68610. & 287.5 & 6.2 & 1.01 \\
\hline-0.31 & -26. & -0.001 & 71528. & 286.8 & 6.9 & 1.01 \\
\hline-0.31 & -26. & -0.001 & 71580. & 286.8 & 6.9 & 1.01 \\
\hline-0.31 & -26 & -0.001 & 71634. & 286.8 & 6.9 & 1.01 \\
\hline-0.31 & -26. & -0.001 & 71682. & 286.8 & 6.9 & 1.01 \\
\hline-0.31 & -26 & -0.001 & 71733. & 286.8 & 6.9 & 1.01 \\
\hline-0.31 & -26 & -0.001 & 71783. & 286.7 & 6.9 & 1.01 \\
\hline-0.31 & -26. & -0.001 & 71837. & 286.7 & 6.9 & 1.01 \\
\hline-0.31 & -26 & -0.001 & 71891. & 286.7 & 7.0 & 1.01 \\
\hline-0.31 & -26 & -0.001 & 71942. & 286.7 & 7.0 & 1.01 \\
\hline-0.31 & -26. & -0.001 & 71992. & 286.7 & 7.0 & 1.01 \\
\hline
\end{tabular}

\begin{tabular}{|c|c|c|c|c|c|c|}
\hline-0.31 & -26. & -0.001 & 72043. & 286.7 & 7.0 & 1.01 \\
\hline-0.31 & -26. & -0.001 & 72097. & 286.7 & 7.0 & 1.01 \\
\hline-0.31 & -26. & -0.001 & 72148. & 286.6 & 7.0 & 1.01 \\
\hline-0.20 & -17. & 0.003 & 72616. & 286.4 & 7.3 & 1.01 \\
\hline-0.20 & -17. & 0.003 & 72666. & 286.4 & 7.3 & 1.01 \\
\hline-0.20 & -17. & 0.003 & 72718. & 286.3 & 7.3 & 1.01 \\
\hline-0.20 & -17. & 0.003 & 72770 . & 286.3 & 7.4 & 1.01 \\
\hline-0.20 & -17. & 0.003 & 72820 . & 286.3 & 7.4 & 1.01 \\
\hline-0.20 & -17. & 0.003 & 72873. & 286.3 & 7.4 & 1.01 \\
\hline-0.20 & -17. & 0.003 & 72925. & 286.2 & 7.4 & 1.01 \\
\hline-0.20 & -17. & 0.003 & 72975. & 286.2 & 7.5 & 1.01 \\
\hline-0.20 & -17. & 0.003 & 73028. & 286.2 & 7.5 & 1.01 \\
\hline-0.20 & -17. & 0.003 & 73077. & 286.2 & 7.5 & 1.01 \\
\hline-0.20 & -17. & 0.003 & 73127. & 286.2 & 7.5 & 1.01 \\
\hline-0.20 & -17. & 0.003 & 73179. & 286.2 & 7.5 & 1.01 \\
\hline-0.20 & -17. & 0.003 & 73234. & 286.2 & 7.5 & 1.01 \\
\hline-0.26 & -23. & 0.001 & 73429. & 286.2 & 7.5 & 1.01 \\
\hline-0.26 & -23. & 0.001 & 73488. & 286.2 & 7.5 & 1.01 \\
\hline-0.26 & -23. & 0.001 & 73548. & 286.2 & 7.5 & 1.01 \\
\hline-0.26 & -23. & 0.001 & 73605. & 286.2 & 7.5 & 1.01 \\
\hline-0.26 & -23. & 0.001 & 73658. & 286.2 & 7.5 & 1.01 \\
\hline-0.26 & -23. & 0.001 & 73711. & 286.2 & 7.5 & 1.01 \\
\hline-0.26 & -23. & 0.001 & 73763. & 286.2 & 7.5 & 1.01 \\
\hline-0.26 & -23. & 0.001 & 73816. & 286.2 & 7.5 & 1.01 \\
\hline-0.26 & -23. & 0.001 & 73870. & 286.2 & 7.5 & 1.01 \\
\hline-0.26 & -23. & 0.001 & 73926. & 286.2 & 7.4 & 1.01 \\
\hline-0.26 & -23. & 0.001 & 73977. & 286.2 & 7.5 & 1.01 \\
\hline-0.26 & -23. & 0.001 & 74036. & 286.2 & 7.4 & 1.01 \\
\hline-0.26 & -23. & 0.001 & 74092. & 286.2 & 7.4 & 1.01 \\
\hline-0.30 & -26. & -0.002 & 77386. & 286.3 & 7.4 & 1.01 \\
\hline-0.30 & -26. & -0.002 & 77439. & 286.3 & 7.4 & 1.01 \\
\hline-0.30 & -26 & -0.002 & 77493. & 286.3 & 7.4 & 1.01 \\
\hline-0.30 & -26. & -0.002 & 77544. & 286.3 & 7.4 & 1.01 \\
\hline-0.30 & -26. & -0.002 & 77595. & 286.3 & 7.4 & 1.01 \\
\hline-0.30 & -26. & -0.002 & 77648. & 286.2 & 7.4 & 1.01 \\
\hline-0.30 & -26. & -0.002 & 77702. & 286.2 & 7.4 & 1.01 \\
\hline-0.30 & -26. & -0.002 & 77752. & 286.2 & 7.5 & 1.01 \\
\hline-0.30 & -26. & -0.002 & 77805. & 286.2 & 7.5 & 1.01 \\
\hline-0.30 & -26. & -0.002 & 77860. & 286.2 & 7.5 & 1.01 \\
\hline-0.30 & -26. & -0.002 & 77911. & 286.1 & 7.5 & 1.01 \\
\hline-0.30 & -26. & -0.002 & 77961. & 286.1 & 7.5 & 1.01 \\
\hline-0.30 & -26. & -0.002 & 78015. & 286.1 & 7.6 & 1.01 \\
\hline-0.29 & -25 & -0.005 & 81839. & 286.3 & 7.4 & 1.01 \\
\hline-0.29 & -25. & -0.005 & 81891. & 286.3 & 7.4 & 1.01 \\
\hline-0.29 & -25. & -0.005 & 81941. & 286.3 & 7.4 & 1.01 \\
\hline-0.29 & -25 & -0.005 & 81992. & 286.3 & 7.3 & 1.01 \\
\hline-0.29 & -25. & -0.005 & 82044. & 286.3 & 7.3 & 1.01 \\
\hline-0.29 & -25 & -0.005 & 82107. & 286.3 & 7.3 & 1.01 \\
\hline-0.29 & -25 & -0.005 & 82161. & 286.3 & 7.3 & 1.01 \\
\hline-0.29 & -25. & -0.005 & 82217. & 286.3 & 7.3 & 1.01 \\
\hline-0.29 & -25 & -0.005 & 82272 . & 286.3 & 7.3 & 1.01 \\
\hline-0.29 & -25. & -0.005 & 82322. & 286.3 & 7.3 & 1.01 \\
\hline-0.29 & -25. & -0.005 & 82372. & 286.3 & 7.3 & 1.01 \\
\hline-0.29 & -25. & -0.005 & 82425. & 286.3 & 7.3 & 1.01 \\
\hline-0.29 & -25. & -0.005 & 82477. & 286.3 & 7.3 & 1.01 \\
\hline-0.36 & -31. & -0.002 & 86294. & 286.5 & 7.2 & 1.01 \\
\hline-0.36 & -31. & -0.002 & 86343. & 286.4 & 7.2 & 1.01 \\
\hline-0.36 & -31. & -0.002 & 86398. & 286.4 & 7.3 & 1.01 \\
\hline-0.36 & -31. & -0.002 & 86447. & 286.4 & 7.2 & 1.01 \\
\hline-0.36 & -31. & -0.002 & 86498. & 286.4 & 7.3 & 1.01 \\
\hline-0.36 & -31. & -0.002 & 86551. & 286.4 & 7.2 & 1.01 \\
\hline-0.36 & -31. & -0.002 & 86603. & 286.4 & 7.2 & 1.01 \\
\hline-0.36 & -31. & -0.002 & 86656. & 286.4 & 7.3 & 1.01 \\
\hline-0.36 & -31. & -0.002 & 86708. & 286.4 & 7.3 & 1.01 \\
\hline-0.36 & -31. & -0.002 & 86759. & 286.4 & 7.3 & 1.01 \\
\hline-0.36 & -31. & -0.002 & 86810 . & 286.4 & 7.3 & 1.01 \\
\hline-0.36 & -31. & -0.002 & 86859. & 286.4 & 7.3 & 1.01 \\
\hline-0.36 & -31. & -0.002 & 86911. & 286.4 & 7.3 & 1.01 \\
\hline-0.31 & -26. & -0.004 & 87078. & 286.3 & 7.3 & 1.01 \\
\hline-0.31 & -26. & -0.004 & 87129. & 286.3 & 7.4 & 1.01 \\
\hline-0.31 & -26. & -0.004 & 87183. & 286.3 & 7.4 & 1.01 \\
\hline-0.31 & -26. & -0.004 & 87237. & 286.3 & 7.4 & 1.01 \\
\hline-0.31 & -26. & -0.004 & 87288. & 286.3 & 7.4 & 1.01 \\
\hline-0.31 & -26. & -0.004 & 87340. & 286.2 & 7.4 & 1.01 \\
\hline-0.31 & -26. & -0.004 & 87395. & 286.2 & 7.5 & 1.01 \\
\hline-0.31 & -26. & -0.004 & 87445. & 286.3 & 7.4 & 1.01 \\
\hline-0.31 & -26. & -0.004 & 87497. & 286.2 & 7.4 & 1.01 \\
\hline-0.31 & -26. & -0.004 & 87548. & 286.2 & 7.4 & 1.01 \\
\hline
\end{tabular}


Table H.2.13 Tap water flushing after $\mathrm{Re}=7000$ contamination tests at $x_{\mathrm{b}}=0.3 \%$ (file:flsh6c2.tb2)

\begin{tabular}{|c|c|c|c|c|c|c|}
\hline $\begin{array}{c}l_{\mathrm{e}} \\
(\mu \mathrm{m})\end{array}$ & $\begin{array}{c}\Gamma \\
\left(\mathrm{kg} / \mathrm{m}^{2}\right) \\
\mathrm{X} 10^{5} \\
\end{array}$ & $\begin{array}{c}x_{\mathrm{b}} \\
(\%) \\
\end{array}$ & $\begin{array}{c}\text { Exposure } \\
\text { Time (s) }\end{array}$ & $\begin{array}{l}\bar{T}_{\mathrm{T}_{\mathrm{T}}} \\
(\mathrm{K})\end{array}$ & $\begin{array}{c}T_{\mathrm{b}}-\bar{T}_{\mathrm{T}_{\mathrm{T}}} \\
(\mathrm{K})\end{array}$ & $\frac{F_{T_{\mathrm{b}}}}{F_{T_{\mathrm{T}}}}$ \\
\hline-0.54 & -46. & 0.003 & 0. & 296.8 & -3.1 & 1.00 \\
\hline-0.54 & -46. & 0.003 & 56. & 296.0 & $\begin{array}{l}-2.3 \\
\end{array}$ & 1.00 \\
\hline-0.54 & -46. & 0.003 & 110. & 295.5 & -1.8 & 1.00 \\
\hline-0.54 & -46. & 0.003 & 165. & 294.9 & -1.1 & 1.00 \\
\hline-0.54 & -46. & 0.003 & 218. & 294.2 & -0.5 & 1.00 \\
\hline-0.54 & -46. & 0.003 & 276. & 293.5 & 0.1 & 1.00 \\
\hline-0.54 & -46. & 0.003 & 328. & 292.9 & 0.8 & 1.00 \\
\hline-0.54 & -46. & 0.003 & 383. & 291.9 & 1.7 & 1.00 \\
\hline-0.54 & -46. & 0.003 & 439. & 290.8 & 2.9 & 1.00 \\
\hline-0.54 & -46. & 0.003 & 492. & 289.9 & 3.8 & 1.01 \\
\hline-0.54 & -46. & 0.003 & 544. & 289.1 & 4.6 & 1.01 \\
\hline-0.54 & -46. & 0.003 & 600 . & 288.4 & 5.3 & 1.01 \\
\hline-0.54 & -46. & 0.003 & 654. & 287.9 & 5.8 & 1.01 \\
\hline-0.03 & -2 & 0.006 & 11606. & 284.2 & 9.5 & 1.01 \\
\hline-0.03 & -2. & 0.006 & 11662. & 284.2 & 9.5 & 1.01 \\
\hline-0.03 & -2. & 0.006 & 11753. & 284.2 & 9.5 & 1.01 \\
\hline-0.03 & -2. & 0.006 & 11809. & 284.2 & 9.5 & 1.01 \\
\hline-0.03 & -2. & 0.006 & 11862. & 284.2 & 9.5 & 1.01 \\
\hline-0.03 & -2. & 0.006 & 11918. & 284.2 & 9.5 & 1.01 \\
\hline-0.03 & -2. & 0.006 & 11974. & 284.2 & 9.5 & 1.01 \\
\hline-0.03 & -2. & 0.006 & 12030. & 284.2 & 9.5 & 1.01 \\
\hline-0.03 & -2. & 0.006 & 12091. & 284.2 & 9.5 & 1.01 \\
\hline-0.03 & -2. & 0.006 & 12143. & 284.2 & 9.5 & 1.01 \\
\hline-0.03 & -2. & 0.006 & 12197. & 284.2 & 9.5 & 1.01 \\
\hline-0.03 & -2. & 0.006 & 12250. & 284.2 & 9.5 & 1.01 \\
\hline-0.03 & -2. & 0.006 & 12305. & 284.1 & 9.5 & 1.01 \\
\hline-0.17 & -15. & 0.005 & 15484. & 283.7 & 10.0 & 1.02 \\
\hline-0.17 & -15. & 0.005 & 15541. & 283.7 & 10.0 & 1.02 \\
\hline-0.17 & -15. & 0.005 & 15599. & 283.7 & 10.0 & 1.02 \\
\hline-0.17 & -15. & 0.005 & 15652. & 283.7 & 10.0 & 1.02 \\
\hline-0.17 & -15. & 0.005 & 15722. & 283.7 & 10.0 & 1.02 \\
\hline-0.17 & -15. & 0.005 & 15778. & 283.7 & 10.0 & 1.02 \\
\hline-0.17 & -15. & 0.005 & 15835. & 283.7 & 10.0 & 1.02 \\
\hline-0.17 & -15. & 0.005 & 15890. & 283.7 & 10.0 & 1.02 \\
\hline-0.17 & -15. & 0.005 & 15953. & 283.7 & 10.0 & 1.02 \\
\hline-0.17 & -15. & 0.005 & 16008. & 283.7 & 10.0 & 1.02 \\
\hline-0.17 & -15. & 0.005 & 16065. & 283.7 & 10.0 & 1.02 \\
\hline-0.17 & -15. & 0.005 & 16120. & 283.7 & 10.0 & 1.02 \\
\hline-0.17 & -15. & 0.005 & 16191. & 283.7 & 10.0 & 1.02 \\
\hline-0.45 & -39. & 0.000 & 77260 . & 285.3 & 8.4 & 1.01 \\
\hline-0.45 & -39. & 0.000 & 77315. & 285.3 & 8.4 & 1.01 \\
\hline-0.45 & -39. & 0.000 & 77369. & 285.3 & 8.4 & 1.01 \\
\hline-0.45 & -39. & 0.000 & 77421. & 285.3 & 8.4 & 1.01 \\
\hline-0.45 & -39. & 0.000 & 77475. & 285.3 & 8.4 & 1.01 \\
\hline-0.45 & -39. & 0.000 & 77526. & 285.3 & 8.4 & 1.01 \\
\hline-0.45 & -39. & 0.000 & 77580 . & 285.3 & 8.4 & 1.01 \\
\hline-0.45 & -39. & 0.000 & 77632. & 285.3 & 8.4 & 1.01 \\
\hline-0.45 & -39. & 0.000 & 77682. & 285.3 & 8.4 & 1.01 \\
\hline-0.45 & -39. & 0.000 & 77735. & 285.3 & 8.4 & 1.01 \\
\hline-0.45 & -39. & 0.000 & 77789. & 285.3 & 8.4 & 1.01 \\
\hline-0.45 & -39. & 0.000 & 77841. & 285.3 & 8.4 & 1.01 \\
\hline-0.45 & -39. & 0.000 & 77895. & 285.3 & 8.4 & 1.01 \\
\hline-0.58 & -50. & -0.009 & 78063. & 285.3 & 8.4 & 1.01 \\
\hline-0.58 & -50. & -0.009 & 78114. & 285.3 & 8.4 & 1.01 \\
\hline-0.58 & -50. & -0.009 & 78168. & 285.2 & 8.4 & 1.01 \\
\hline-0.58 & -50. & -0.009 & 78219. & 285.2 & 8.5 & 1.01 \\
\hline-0.58 & -50. & -0.009 & 78274. & 285.2 & 8.5 & 1.01 \\
\hline-0.58 & -50. & -0.009 & 78327. & 285.2 & 8.5 & 1.01 \\
\hline-0.58 & -50. & -0.009 & 78381. & 285.2 & 8.5 & 1.01 \\
\hline-0.58 & -50. & -0.009 & 78433. & 285.2 & 8.5 & 1.01 \\
\hline-0.58 & -50. & -0.009 & 78485. & 285.2 & 8.5 & 1.01 \\
\hline-0.58 & -50. & -0.009 & 78539. & 285.2 & 8.5 & 1.01 \\
\hline-0.58 & -50. & -0.009 & 78593. & 285.2 & 8.5 & 1.01 \\
\hline-0.58 & -50. & -0.009 & 78647. & 285.2 & 8.5 & 1.01 \\
\hline-0.58 & -50. & -0.009 & 78701. & 285.2 & 8.5 & 1.01 \\
\hline-0.66 & -56. & -0.013 & 82130. & 285.0 & 8.7 & 1.01 \\
\hline-0.66 & -56. & -0.013 & 82181. & 285.0 & 8.8 & 1.01 \\
\hline-0.66 & -56. & -0.013 & 82238. & 285.0 & 8.7 & 1.01 \\
\hline-0.66 & -56. & -0.013 & 82292. & 284.9 & 8.8 & 1.01 \\
\hline-0.66 & -56. & -0.013 & 82350 . & 284.9 & 8.8 & 1.01 \\
\hline-0.66 & -56. & -0.013 & 82404. & 284.9 & 8.8 & 1.01 \\
\hline-0.66 & -56. & -0.013 & 82457. & 284.9 & 8.8 & 1.01 \\
\hline-0.66 & -56. & -0.013 & 82512. & 284.9 & 8.8 & 1.01 \\
\hline-0.66 & -56. & -0.013 & 82565. & 284.9 & 8.8 & 1.01 \\
\hline-0.66 & -56. & -0.013 & 82616. & 284.9 & 8.8 & 1.01 \\
\hline-0.66 & -56. & -0.013 & 82670. & 284.9 & 8.8 & 1.01 \\
\hline-0.66 & -56. & -0.013 & 82723. & 284.9 & 8.8 & 1.01 \\
\hline
\end{tabular}

\begin{tabular}{|c|c|c|c|c|c|c|}
\hline-0.66 & -56. & -0.013 & 82774. & 284.9 & 8.8 & 1.01 \\
\hline-0.58 & -50. & -0.005 & 86376. & 284.1 & 9.6 & 1.01 \\
\hline-0.58 & -50. & -0.005 & 86430. & 284.1 & 9.6 & 1.01 \\
\hline-0.58 & -50. & -0.005 & 86481. & 284.1 & 9.6 & 1.01 \\
\hline-0.58 & -50. & -0.005 & 86533. & 284.2 & 9.5 & 1.01 \\
\hline-0.58 & -50. & -0.005 & 86587. & 284.2 & 9.5 & 1.01 \\
\hline-0.58 & -50. & -0.005 & 86642. & 284.1 & 9.5 & 1.01 \\
\hline-0.58 & -50. & -0.005 & 86701. & 284.2 & 9.5 & 1.01 \\
\hline-0.58 & -50. & -0.005 & 86757. & 284.2 & 9.5 & 1.01 \\
\hline-0.58 & -50. & -0.005 & 86811. & 284.2 & 9.5 & 1.01 \\
\hline-0.58 & -50. & -0.005 & 86865. & 284.2 & 9.5 & 1.01 \\
\hline-0.58 & -50. & -0.005 & 86918. & 284.2 & 9.5 & 1.01 \\
\hline-0.58 & -50 & -0.005 & 86972. & 284.1 & 9.5 & 1.01 \\
\hline-0.58 & -50. & -0.005 & 87025 . & 284.2 & 9.5 & 1.01 \\
\hline-0.56 & -48. & -0.007 & 87172. & 284.1 & 9.6 & 1.01 \\
\hline-0.56 & -48. & -0.007 & 87225 . & 284.1 & 9.5 & 1.01 \\
\hline-0.56 & -48. & -0.007 & 87278. & 284.1 & 9.6 & 1.01 \\
\hline-0.56 & -48. & -0.007 & 87335. & 284.1 & 9.6 & 1.02 \\
\hline-0.56 & -48. & -0.007 & 87400 . & 284.1 & 9.6 & 1.01 \\
\hline-0.56 & -48. & -0.007 & 87456. & 284.1 & 9.6 & 1.01 \\
\hline-0.56 & -48. & -0.007 & 87513. & 284.1 & 9.6 & 1.01 \\
\hline-0.56 & -48. & -0.007 & 87565 . & 284.1 & 9.6 & 1.01 \\
\hline-0.56 & -48. & -0.007 & 87621. & 284.1 & 9.6 & 1.02 \\
\hline-0.56 & -48. & -0.007 & 87672. & 284.1 & 9.6 & 1.02 \\
\hline-0.56 & -48. & -0.007 & 87727. & 284.1 & 9.6 & 1.02 \\
\hline-0.56 & -48. & -0.007 & 87780 . & 284.1 & 9.6 & 1.02 \\
\hline-0.56 & -48. & -0.007 & 87832. & 284.1 & 9.6 & 1.02 \\
\hline-0.50 & -43. & -0.003 & 87988. & 284.1 & 9.6 & 1.02 \\
\hline-0.50 & -43. & -0.003 & 88044 . & 284.1 & 9.6 & 1.02 \\
\hline-0.50 & -43. & -0.003 & 88098. & 284.0 & 9.7 & 1.02 \\
\hline-0.50 & -43. & -0.003 & 88154. & 284.0 & 9.7 & 1.02 \\
\hline-0.50 & -43. & -0.003 & 88207. & 284.0 & 9.7 & 1.02 \\
\hline-0.50 & -43. & -0.003 & 88265 . & 284.1 & 9.6 & 1.02 \\
\hline-0.50 & -43. & -0.003 & 88318. & 284.1 & 9.6 & 1.02 \\
\hline-0.50 & -43. & -0.003 & 88371. & 284.0 & 9.6 & 1.02 \\
\hline-0.50 & -43. & -0.003 & 88426 . & 284.0 & 9.7 & 1.02 \\
\hline-0.50 & -43. & -0.003 & 88480 . & 284.0 & 9.7 & 1.02 \\
\hline-0.50 & -43. & -0.003 & 88538. & 284.0 & 9.6 & 1.02 \\
\hline-0.50 & -43. & -0.003 & 88593. & 284.0 & 9.7 & 1.02 \\
\hline-0.50 & -43. & -0.003 & 88648. & 284.0 & 9.7 & 1.02 \\
\hline-0.66 & -57. & -0.011 & 95135. & 284.2 & 9.6 & 1.01 \\
\hline-0.66 & -57. & -0.011 & 95191. & 284.2 & 9.6 & 1.01 \\
\hline-0.66 & -57. & -0.011 & 95245. & 284.2 & 9.5 & 1.01 \\
\hline-0.66 & -57. & -0.011 & 95298. & 284.2 & 9.5 & 1.01 \\
\hline-0.66 & -57. & -0.011 & 95357. & 284.2 & 9.5 & 1.01 \\
\hline-0.66 & -57. & -0.011 & 95413. & 284.2 & 9.5 & 1.01 \\
\hline-0.66 & -57. & -0.011 & 95472. & 284.2 & 9.4 & 1.01 \\
\hline-0.66 & -57. & -0.011 & 95529. & 284.2 & 9.4 & 1.01 \\
\hline-0.66 & -57. & -0.011 & 95584. & 284.3 & 9.4 & 1.01 \\
\hline-0.66 & -57. & -0.011 & 95635. & 284.3 & 9.4 & 1.01 \\
\hline-0.66 & -57. & -0.011 & 95691. & 284.3 & 9.4 & 1.01 \\
\hline-0.66 & -57. & -0.011 & 95746. & 284.3 & 9.4 & 1.01 \\
\hline-0.66 & -57. & -0.011 & 95801. & 284.3 & 9.4 & 1.01 \\
\hline-0.52 & -44. & -0.011 & 100071. & 284.3 & 9.4 & 1.01 \\
\hline-0.52 & -44. & -0.011 & 100129. & 284.3 & 9.4 & 1.01 \\
\hline-0.52 & -44. & -0.011 & 100183. & 284.3 & 9.4 & 1.01 \\
\hline-0.52 & -44. & -0.011 & 100237. & 284.3 & 9.4 & 1.01 \\
\hline-0.52 & -44. & -0.011 & 100290 . & 284.3 & 9.4 & 1.01 \\
\hline-0.52 & -44. & -0.011 & 100344. & 284.2 & 9.4 & 1.01 \\
\hline-0.52 & -44 & -0.011 & 100398. & 284.3 & 9.4 & 1.01 \\
\hline-0.52 & -44. & -0.011 & 100453. & 284.2 & 9.4 & 1.01 \\
\hline-0.52 & -44. & -0.011 & 100515. & 284.2 & 9.5 & 1.01 \\
\hline-0.52 & -44 & -0.011 & 100575 . & 284.2 & 9.5 & 1.01 \\
\hline-0.52 & -44. & -0.011 & 100631. & 284.1 & 9.6 & 1.01 \\
\hline-0.52 & -44. & -0.011 & 100689. & 284.1 & 9.5 & 1.01 \\
\hline-0.52 & -44 & -0.011 & 100744. & 284.2 & 9.5 & 1.01 \\
\hline-0.71 & -61. & -0.010 & 166191. & 285.9 & 7.8 & 1.01 \\
\hline-0.71 & -61. & -0.010 & 166248. & 285.8 & 7.8 & 1.01 \\
\hline-0.71 & -61. & -0.010 & 166302. & 285.9 & 7.8 & 1.01 \\
\hline-0.71 & -61. & -0.010 & 166356. & 285.9 & 7.8 & 1.01 \\
\hline-0.71 & -61. & -0.010 & 166410. & 285.9 & 7.8 & 1.01 \\
\hline-0.71 & -61. & -0.010 & 166464. & 285.9 & 7.8 & 1.01 \\
\hline-0.71 & -61. & -0.010 & 166518. & 285.9 & 7.8 & 1.01 \\
\hline-0.71 & -61. & -0.010 & 166574. & 285.9 & 7.8 & 1.01 \\
\hline-0.71 & -61. & -0.010 & 166626. & 285.9 & 7.8 & 1.01 \\
\hline-0.71 & -61. & -0.010 & 166676. & 285.9 & 7.8 & 1.01 \\
\hline-0.71 & -61. & -0.010 & 166727. & 285.9 & 7.8 & 1.01 \\
\hline-0.71 & -61. & -0.010 & 166779. & 285.9 & 7.8 & 1.01 \\
\hline-0.71 & -61. & -0.010 & 166832. & 285.9 & 7.8 & 1.01 \\
\hline-0.56 & -48. & -0.007 & 168424. & 285.4 & 8.3 & 1.01 \\
\hline
\end{tabular}




\section{APPENDIX I: SPECTROFLUOROMETER CHECK}

This appendix discusses how the emission and excitation wavelength measurements were verified with a mercury standard and a "crossover peak" from the excitation. The emission wavelength measurement obtained from the spectrofluorometer without the glass filter was checked against a mercury vapor light. Figure I.1 and Table I.1 show a comparison of the published values of the peak wavelengths for mercury (Reader et al., 1980) to those obtained from the spectrofluorometer. The absolute difference between the measured and published wavelengths was approximately within $10 \mathrm{~nm}$.

The excitation wavelength measurement obtained from the spectrofluorometer was checked with a "crossover peak" from the excitation. In other words, the excitation monochromator was set to a specific wavelength with no specimen in the sample chamber. Under these conditions, the emission intensity should peak at the excitation wavelength. The wavelength of the emission peaked at the excitation wavelength to within the resolution of the digital display ( $\pm 1 \mathrm{~nm}$ ) for the wavelengths that were tested.

Table I.1 Calibration check of spectrofluorometer against Mercury lamp

\begin{tabular}{|c|c|c|c|}
\hline $\begin{array}{c}\text { Published } \\
\text { wavelength } \\
(\mathrm{nm})\end{array}$ & $\begin{array}{c}\text { Measured } \\
\text { wavelength } \\
(\mathrm{nm})\end{array}$ & $\begin{array}{c}\text { Absolute } \\
\text { difference } \\
(\mathrm{nm})\end{array}$ & $\begin{array}{c}\text { Relative } \\
\text { Difference } \\
(\%)\end{array}$ \\
\hline 312.567 & 307 & 5 & 1.6 \\
\hline 365.015 & 358 & 7 & 1.9 \\
\hline 404.656 & 398 & 7 & 1.7 \\
\hline 435.833 & 427 & 9 & 2.1 \\
\hline 546.074 & 540 & 6 & 1.1 \\
\hline 576.960 & 569 & 8 & 1.4 \\
\hline
\end{tabular}

${ }^{1}$ Reader et al. (1980) 


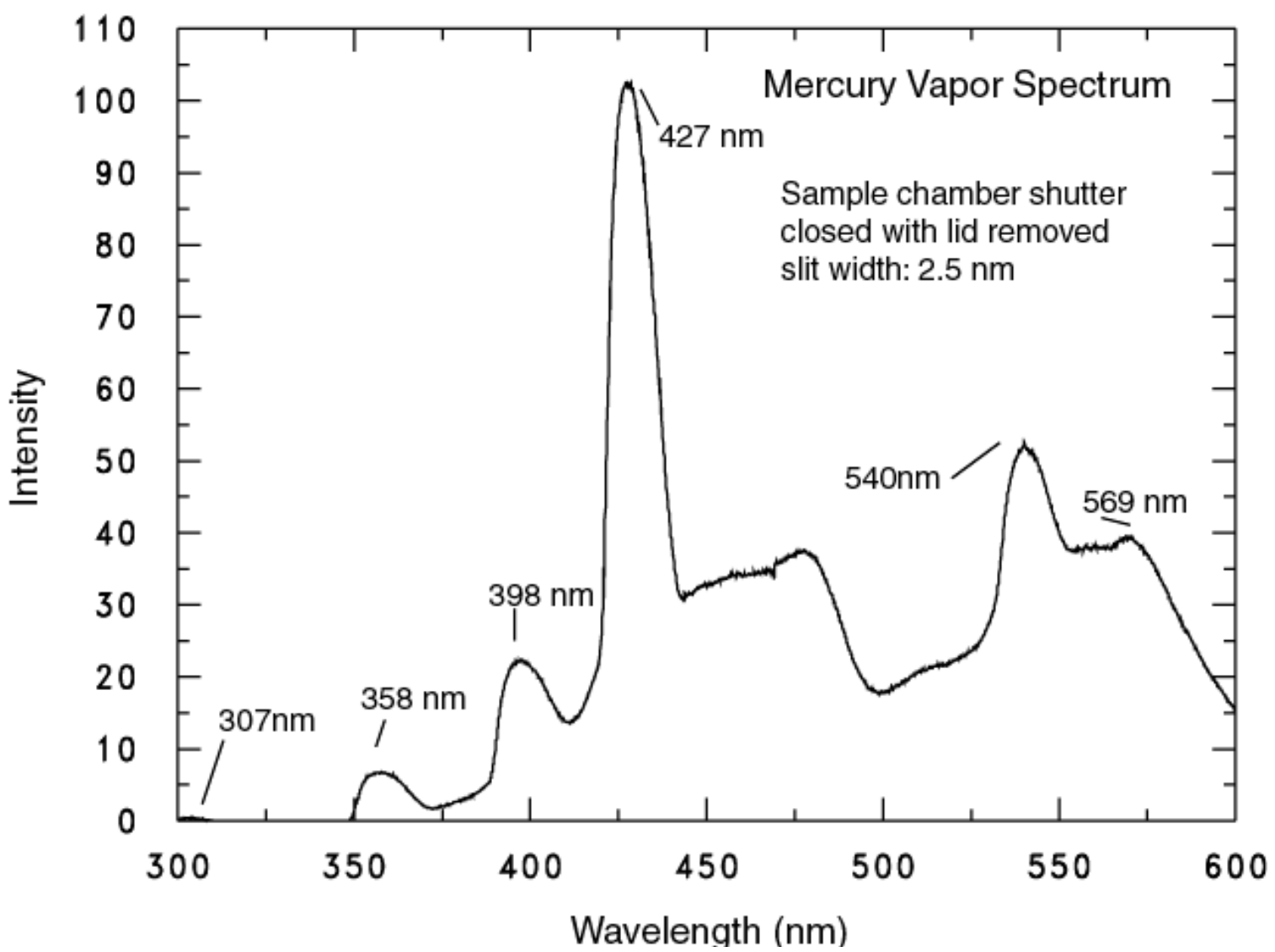

Fig. I.1 Verification of spectrofluorometer wavelength with Mercury standard 